\author{
Universidade de São Paulo \\ Instituto de Física
}

\title{
Espectroscopia de Absorção de Dois Fótons em Moléculas Orgânicas Incluindo Efeitos de Solvente
}

\author{
Tárcius Nascimento Ramos
}

Orientador: Prof. Dr. Sylvio Roberto Accioly Canuto

Coorientador: Prof. Dr. Daniel Luiz da Silva

Tese de doutorado apresentada ao Instituto de Física como requisito parcial para a obtenção do título de Doutor em Ciências.

Banca Examinadora:

Prof. Dr. Sylvio Roberto Accioly Canuto (IF/USP)

Prof. Dr. Antonio Carlos Borin (IQ/USP)

Prof. Dr. Caetano Rodrigues Miranda (IF/USP)

Prof. Dr. Marcos Antônio de Castro (IF/UFG)

Profa. Dra. Paula Homem-de-Mello (UFABC) 


\section{FICHA CATALOGRÁFICA \\ Preparada pelo Serviço de Biblioteca e Informação do Instituto de Física da Universidade de São Paulo}

Ramos, Tárcius Nascimento

Espectroscopia de absorção de dois fótons em moléculas orgânicas incluindo efeitos de solvente. São Paulo, 2020.

Tese (Doutorado) - Universidade de São Paulo. Instituto de Física. Depto. de Física Geral

Orientador: Prof. Dr. Sylvio Roberto Accioly Canuto

Área de Concentração: Física

Unitermos: 1. Óptica não linear; 2. Estrutura eletrônica; 3. Absorção; 4. Solvatação.

USP/IF/SBI-027/2020 


\title{
University of São Paulo \\ Physics Institute
}

\section{Two-Photon Absorption Spectroscopy in Organic Molecules Including Solvent Effects}

\author{
Tárcius Nascimento Ramos
}

Supervisor: $\quad$ Prof. Dr. Sylvio Roberto Accioly Canuto

Co-supervisor: Prof. Dr. Daniel Luiz da Silva

Thesis submitted to the Physics Institute of the University of São Paulo in partial fulfillment of the requirements for the degree of Doctor of Science.

Examining Committee:

Prof. Dr. Sylvio Roberto Accioly Canuto (IF/USP)

Prof. Dr. Antonio Carlos Borin (IQ/USP)

Prof. Dr. Caetano Rodrigues Miranda (IF/USP)

Prof. Dr. Marcos Antônio de Castro (IF/UFG)

Profa. Dra. Paula Homem-de-Mello (UFABC) 

Aos meus pais e meu irmão, que sempre me apoiaram e me motivaram a vencer todos os desafios. 


\section{AgradeCimentos}

Ao Criador que proporcionou a oportunidade de me manifestar nesse mundo cheio de mistérios encantadores.

À tia Inês pelo acolhimento, carinho, convivência.

À minha família por todo o apoio, carinho, paciência durante essa jornada.

À minha noiva Taís Porcari por tudo.

Aos orientadores Prof. Sylvio Canuto e Prof. Daniel da Silva pelo acolhimento e dedicação e ao Prof. Benoît Champagne.

Aos amigos pelas produtivas discussões sobre espiritualidade, ciência, convivência, respeito. Aos membros do Grupo de Física Molecular e Modelagem e do Laboratoire de Chimie Théorique.

A todos os funcionários da USP pela agradável convivência.

O presente trabalho foi realizado com apoio da Coordenação de Aperfeiçoamento de Pessoal de Nível Superior - Brasil (CAPES) - Código de Financiamento 001

À FAPESP pelo apoio financeiro durante o doutorado (processo $n^{\circ}$ 2015/14189-3, Fundação de Amparo à Pesquisa do Estado de São Paulo (FAPESP)), durante o estágio no exterior (processo n 2018/17453-1, Fundação de Amparo à Pesquisa do Estado de São Paulo (FAPESP)) e nos demais apoios desde a iniciação científica. 
"So close no matter how far

Couldn't be much more from the heart

Forever trusting who we are

And nothing else matters"

Nothing Else Matters - Metallica

"Se você exerce a sua vocação,

metade da sua vida está resolvida"

Fernanda Montenegro 


\section{PARTICIPAÇÃo EM EVENTOS}

1. XLV Congress of Theoretical Chemists of Latin Expression. Theoretical description of the first and second hyperpolarizabilities responses of ion pairs in solution. Montreal, Canadá - 2019. (Congresso).

2. XLIV Congress of Theoretical Chemists of Latin Expression. Theoretical one- and twophoton absorption characterization of water-soluble bioimaging probe. Santiago, Chile, 2018. (Congresso).

3. April Meeting 2018. The broadening effects on para-Nitroaniline two-photon absorption cross-section. Columbus, Estados Unidos da América - 2018. (Congresso).

4. XIX Simpósio Brasileiro de Química Teórica. The role of charge transfer in the two-photon absorption cross-section. Águas de Lindóia, SP, Brasil - 2017. (Simpósio).

5. XL Encontro Nacional de Física da Matéria Condensada. Modelling two-photon spectroscopy in liquid environments. Case study of the p-Nitroaniline. Armação de Búzios, RJ, Brasil - 2017. (Encontro).

6. CAPES-STINT Second Workshop Natal. Solvent Effects on Molecular Properties and Spectroscopy. Natal, RN, Brasil - 2016. (Encontro).

7. Encontro de Física 2016. Two-photon absorption of p-Nitroaniline in liquid environments. Natal, RN, Brasil - 2016. (Encontro).

8. II Workshop on Biomolecular Theory-Experimental Interplay. Solvent Effects on Different Electronic States of Dimethoxy Curcumin. Maresias, SP, Brasil - 2015. (Congresso).

9. Reunião Anual do NAP-FCx 2015. Efeitos de solventes em diferentes estados eletrônicos da Dimethoxy Curcumin. Atibaia, SP, Brasil - 2015. (Encontro).

\section{ESTÁGIO NO EXTERIOR}

Realização de estágio no exterior por período de seis meses (novembro de 2018 a abril de 2019) sob a supervisão do Prof. Dr. Benoît Champagne na Universidade de Namur - Bélgica. Projeto intitulado "QM/MM Investigation of the Electric Field-Induced Second Harmonic Generation (EFISHG) Response of Ion Pairs in Solution”. 


\section{ProduÇão durante o doUTORAdo}

1. Bistafa, C.; Ramos, T. N.; Coutinho, K.; Canuto, S. Quantum mechanics meets scaling theory near the critical point (submetido).

2. Ramos, T. N.; Canuto, S.; Champagne, B. Unraveling the electric field induced second harmonic generation responses of stilbazolium ion pairs complexes in solution using a multiscale simulation method (submetido).

3. Ramos, T. N.; Silva, D. L.; Cabral, B. J. C.; Canuto, S. On the Spectral Line Width Broadening for Simulation of the Two-Photon Absorption Cross-Section of ParaNitroaniline in Liquid Environment. J. Mol. Liq. 2020, 301, 112405. https://doi.org/10.1016/j.molliq.2019.112405.

4. MUSPEC - Multiconfigurational Spectrum Convolution; Um programa para construir espectros teóricos utilizando centenas de valores de energia e intensidade provenientes de amostragem de cálculos S-QM/MM. https://github.com/tarciusnramos/muspec.

5. Abegão, L. M. G.; Fonseca, R. D.; Ramos, T. N.; Mahuteau-Betzer, F.; Piguel, S.; Joatan, J. R.; Mendonça, C. R.; Canuto, S.; Silva, D. L.; De Boni, L. Oxazole Dyes with Potential for Photoluminescence Bioprobes: A Two-Photon Absorption Study. J. Phys. Chem. C 2018, 122 (19), 10526-10534. https://doi.org/10.1021/acs.jpcc.8b01904.

6. Ramos, T. N.; Canuto, S. A Theoretical Study of the Low-Lying Excited States and the Photophysics of Dimethoxy Curcumin in Cyclohexane and Acetonitrile. Theor. Chem. Acc. 2017, 136 (7), 78. https://doi.org/10.1007/s00214-017-2108-9. 



\section{RESUMO}

As propriedades eletrônicas relacionadas com o processo de absorção de dois fótons (A2F) em moléculas orgânicas isoladas e em meio líquido foram estudadas buscando caracterizar as transições eletrônicas, modular a intensidade da $\mathrm{A} 2 \mathrm{~F}$ e propor uma forma de atribuir o valor ao parâmetro de alargamento espectral, incluindo os efeitos de solventes. Os efeitos de solventes foram incluídos utilizando solvatação explícita com Born-Oppenheimer Molecular Dynamics, Dinâmica Molecular Clássica e amostragem de Monte Carlo Metropolis além da solvatação implícita com Polarizable Continuum Model. Utilizando a metodologia Sequential-Quantum Mechanics / Molecular Mechanics avaliamos diferentes procedimentos para convoluir o espectro de A2F e obter a respectiva seção de choque de A2F. Considerando a contribuição das configurações extraídas das simulações moleculares propomos um ajuste da largura total da banda de A2F. Deste ajuste, resultam as melhores descrições do valor máximo e da intensidade relativa da seção de choque de A2F da molécula para-Nitroanilina em ambientes líquidos. Uma estratégia utilizando campos eletrostáticos externos para modular a A2F também foi explorada em duas betaínas. A presença do campo eletrostático realçou a intensidade da seção de choque de $\mathrm{A} 2 \mathrm{~F}$ ao mesmo tempo que reduziu a probabilidade de absorção de um fóton (A1F), evidenciando potencial utilização para evitar efeitos espúrios provenientes da A1F em aplicações do processo de A2F. Mostramos para sistemas moleculares grandes que a utilização de métodos semi-empíricos, os quais apresentam baixo custo computacional, pode fornecer descrições mais precisas para as intensidades de A2F, em particular quando excitações duplas são incluídas. Por fim, utilizando simulações moleculares de complexos neutros constituídos por pares iônicos mostramos que a resposta Electric FieldInduced Second Harmonic Generation é modulada pela posição relativa do ânion. Esta dependência ocorre principalmente devido às variações do momento de dipolo e não devido às variações das componentes da primeira hiperpolarizabilidade.

Palavras-chave: Absorção de dois fótons, EFISHG, solvatação, S-QM/MM. 



\section{Abstract}

The electronic properties related with the two-photon absorption process (TPA) in isolated or solvated organic molecules were studied aiming at characterizing the electronic transitions, tune the TPA intensity and to suggest a way to assign the value of the spectral broadening parameter, including solvent effects. The solvent effects were included using explicit solvation with Born-Oppenheimer Molecular Dynamics, Classical Molecular Dynamics and Monte Carlo Metropolis sampling as well the implicit solvation with Polarizable Continuum Model. Employing the Sequential-Quantum Mechanics / Molecular Mechanics we evaluate different procedures to convolute the TPA spectrum and to obtain the respective TPA cross-section. Considering the contributions of sampled configurations from molecular simulations we suggest a fit of the full width at half maximum of the TPA band. This fit resulted in better description of the maximum value and the relative intensity of the TPA cross-section of the para-Nitroaniline in liquid environments. A strategy using external electrostatic fields enhanced the TPA cross-section was also explored for two betaines. The presence of the field reduced the transition probability of the one-photon absorption (OPA), highlighting the potential use to avoid spurious effects from OPA in the TPA process applications. We showed for large molecular systems that using semi empirical methods, which are computationally cheap, can provide more accurate descriptions for the TPA intensities, especially when the double excitations are included in the calculations. Using molecular simulations of ion pairs as neutral complexes we showed that the Electric Field-Induced Second Harmonic Generation response is tuned by the anion relative position. This dependence become of the anion ability to change the dipole moment and it is not related with changing the first-hyperpolarizability tensor components.

Keywords: Two-photon absorption, EFISHG, solvation effects, S-QM/MM. 


\section{LISTAS DE FIGURAS}

Figura 1. Diagrama de absorção de um fóton de energia $\omega 1$ e de dois fótons de energias $\omega 1$ e $\omega 2 \ldots . .20$ Figura 2. (a) Representação de um disco óptico de armazenamento tridimensional (em amarelo) e das trilhas de gravação (em laranja) [23]. (b) Microfabricação do Cristo Redentor em material polimérico [24]. (c) Resolução da fluorescência promovida pelos processos de absorção de um e de dois fótons

[25]. (d) bioimagiamento da concentração de cloreto em glândulas salivares [26]. 21

Figura 3. Representação diagramática dos níveis de energia molecular (linhas contínuas), dos estados virtuais (linhas tracejadas) e dos processos de absorção e emissão de fótons (setas). $\omega$ representa a energia do fóton e $\Delta E$ a diferença entre as energias do estado $\mid n$ e do estado virtual...

Figura 4. Modelo de cavitação baseado em uma única esfera (lado esquerdo) e na superposição de esferas (lado direito) centradas em cada átomo. 51

Figura 5. Fluxograma indicando a sequência de cálculos realizados no método S-QM/MM. .55

Figura 6. Ilustração dos efeitos dos diferentes procedimentos na largura de linha do espectro total.... 63 Figura 7. Orbitais de KS envolvidos na primeira transição intensa para os processos de A1F e A2F. 65 Figura 8. Comparação da largura de linha total obtida para o espectro de A2F com o valor experimental relacionado com o espectro de A1F.

Figura 9. Espectros de A2F obtidos considerando os cinco procedimentos adotados e utilizando as aproximações PCM e ASEC para o solvente. Os dados experimentais foram extraídos da referência [127].

Figura 10: Estrutura química da (a) para-betaína e (b) ortho-betaína.

Figura 11. Seção de choque de absorção de dois fótons em função do ângulo torcional $\phi$ entre os anéis para a para-betaína (esquerda) e ortho-betaína (direita)..... .75

Figura 12. Propriedades eletrônicas diretamente relacionadas com o modelo de dois níveis para a seção de choque de A2F em função do ângulo $\phi$ entre os anéis. Energia de transição $\Delta E$, dipolo de transição $\mu g e$, dipolo permanente do estado fundamental $\mu g g$ e dipolo permanente do estado excitado нee.

Figura 13. Quantidade de carga $\mathrm{q}_{\mathrm{t}}$ transferida durante a excitação e distância de deslocamento R entre os pontos de decréscimo e acréscimo da carga em função do ângulo $\phi$ entre os anéis. .77 Figura 14. Orbitais moleculares envolvidos na excitação eletrônica da pBT obtidos quando $\phi$ assume os valores de 0,30 e $90^{\circ}$.

Figura 15. Seção de choque de absorção de dois fótons $\sigma$ obtida utilizando o modelo de dois níveis e teoria de resposta quadrática em função da carga $\mathrm{q}_{\mathrm{t}}$ transferida durante a excitação eletrônica. .79 
Tárcius N. Ramos

Figura 16. Energia de transição $\Delta E$, momento de dipolo permanente do estado fundamental $\mu g g$ e do estado excitado $\mu e e$ e momento de dipolo de transição entre o estado fundamental e excitado $\mu g e$ obtidos para a pBT (esquerda) e para a oBT (direita) em diferentes valores de intensidade de campo elétrico. $\mathrm{G}_{\mathrm{F}}$ : Geometria obtida incluindo efeitos do campo F; $\mathrm{G}_{\mathrm{R}}$ : Geometria obtida na condição de molécula isolada; wF: Propriedades eletrônicas obtidas considerando o campo F; nF: Propriedades eletrônicas obtidas na condição de molécula isolada.

Figura 17. Quantidade de carga transferida durante a excitação $\mathrm{q}_{\mathrm{t}}$ e distância de deslocamento R entre os pontos de decréscimo e acréscimo da carga obtidas para a pBT (esquerda) e para a oBT (direita) em diferentes valores de intensidade de campo elétrico. $\mathrm{G}_{\mathrm{F}}$ : Geometria obtida incluindo efeitos do campo F; $\mathrm{G}_{\mathrm{R}}$ : Geometria obtida na condição de molécula isolada; $\mathrm{wF}$ : Propriedades eletrônicas obtidas considerando o campo F; nF: Propriedades eletrônicas obtidas na condição de molécula isolada.

Figura 18. Centros de decréscimo e acréscimo de cargas (superior) e diferença das densidades eletrônicas entre os estados fundamental e excitado (inferior) para a pBT. A cor ciano representa a densidade negativa e a roxa a densidade positiva. A unidade de $\mathrm{F}$ é $10^{-4} \mathrm{u}$.a.

Figura 19. Seção de choque de A2F obtidas para a pBT (esquerda) e para a oBT (direita) em diferentes valores de intensidade de campo elétrico. $\mathrm{G}_{\mathrm{F}}$ : Geometria obtida incluindo efeitos do campo $\mathrm{F} ; \mathrm{G}_{\mathrm{R}}$ :

Geometria obtida na condição de molécula isolada; wF: Propriedades eletrônicas obtidas considerando o campo F; nF: Propriedades eletrônicas obtidas na condição de molécula isolada. 83

Figura 20. Estrutura química dos compostos 7 (em azul) e 10 (em vermelho) [160,161]. 87

Figura 21. Seção de choque de A2F. Adaptado da referência [161]. 88

Figura 22. Diagrama de energia dos orbitais moleculares do composto 10. As cores indicam a energia dos orbitais moleculares: vermelho - incluídos nos cálculos com excitações duplas, azuis - incluídos nos cálculos com excitações simples e pretas somente por referência.

Figura 23. Geometrias de mínima energia obtidas com B3LYP/6-31G(d) para os compostos isolados.

Figura 24. Espectros vibracionais infravermelho (IR) e Raman obtidos com B3LYP/6-31G(d) para os compostos isolados.

Figura 25. Geometrias sem as cadeias hidrofílicas. Chamadas neste texto de geometrias reduzidas... 91 Figura 26. Espectros de A1F obtidos com diferentes funcionais DFT para os compostos completos (linhas cheias) e para os compostos reduzidos (linhas pontilhadas). As intensidades foram normalizadas em relação aos máximos obtidos com B3LYP para os sistemas completos. Os dados experimentais foram obtidos em solução aquosa em todos os painéis.

Figura 27. Simulação do espectro de A1F comparando os compostos completo (linhas cheias) e reduzido (linhas pontilhadas). Os espaços ativos selecionados foram: 7 - OC=31(31) e $\mathrm{NO}=40(34) ; 10$ - $\mathrm{OC}=31(34)$ e $\mathrm{NO}=43(38)$ para compostos completos (reduzidos). Adotamos $\Gamma=0.6 \mathrm{eV}$.

Figura 28. Simulação do espectro de A2F com diferentes funcionais DFT e com o método semiempírico considerando excitações simples e duplas utilizando a expressão soma-sobre-estados. Os 
resultados dos compostos completos são apresentados em linhas contínuas e dos compostos reduzidos em linhas pontilhadas, exceto para os últimos quadros, que incluem os efeitos de solventes com PCM, onde as linhas contínuas representam os resultados dos compostos reduzidos. Todos os espectros foram construídos utilizando $\Gamma=0.4 \mathrm{eV}$

Figura 29. Orbitais moleculares envolvidos nos processos de absorção de um e de dois fótons obtidos com CAM-B3LYP para o composto 10. A seta azul indica o processo de A1F e a seta vermelha indica o processo de $\mathrm{A} 2 \mathrm{~F}$. 98

Figura 30. Variação do resultado obtido com o método soma-sobre-estados considerando 50, 100, 150 e 200 estados intermediários para o composto 10. Considerando apenas excitações simples (esquerda) e excitações simples e duplas (direita). 100

Figura 31. Estrutura do cátion stilbazolium e lista dos ânions. *p-toluenosulfonato. 102 Figura 32.(a) Posição relativa do $\mathrm{I}^{-}$e (b) do $\mathrm{pTS}^{-}$ao redor do cátion do complexo 4. As geometrias dos cátions representam única configuração enquanto as distribuições dos ânions representam a sobreposição de 200 configurações. 106

Figura 33. Diferença das densidades eletrônicas dos estados excitado e fundamental $(\Delta \rho=\rho e x-$ $\rho g r$ ) para os complexos $\mathbf{1}$ e $\mathbf{3}$ junto com as respectivas densidades $\rho$ ex e $\rho g r . \Delta \rho$ está representado por isosuperfícies de valor 0.0004 onde a cor azul corresponde a região de decréscimo da densidade eletrônica e a cor violeta representa a região de acréscimo da densidade eletrônica. 108 Figura 34 Distribuição violino dos resultados de $\mu \beta / /$ e da componente $\mu \beta / / x$ para os diferentes complexos, incluindo os valores médios (pontos pretos) e os desvios padrão (linhas pretas). Figura 35. Distribuição violino dos resultados para (a) $\mu \beta / /$ e (b) $\beta H R S$, incluindo os valores médios (pontos pretos), desvio padrão (linhas pretas) e diagrama de caixa (linhas azuis indicando o primeiro quartil $25 \%$, a mediana e o terceiro quartil $75 \%$ ) para o complexo 1

Figura 36. Correlação linear para propriedades moleculares $(\beta x, \beta H R S, \Delta E$, f e $\mu x)$ e os parâmetros geométricos (BLA e a projeção da coordenada $\mathrm{X}_{\mathrm{NA}}$ da distância $\mathrm{d}_{\mathrm{NA}}$ )

Figura 37. Razão entre as respostas EFISHG considerando ou não os efeitos de terceira ordem (em azul) e o termo de terceira ordem $\gamma / /$ (em laranja).....

Figura 38. Convolução do espectro de absorção linear considerando funções Lorentzianas para 3 grupos: (1) estado 1 e $\Gamma=0.6$; (2) estado 2 e $\Gamma=0.35$; e (3) estados 3 a 10 e $\Gamma=0.2$.

Figura 39. Análise dos picos e larguras de linha do espectro obtidos para cada grupo e para o espectro total referente ao espectro apresentado na Figura 38.

Figura 40. Formato do arquivo de exportação. As colunas são separadas por grupos e o na última coluna as intensidades do espectro total. 


\section{LISTAS TABELAS}

Tabela 1. Processos ópticos não lineares frequentemente analisados. Adaptado da referência [50] ... 46 Tabela 2. Resultados obtidos utilizando funções respostas de primeira, segunda e terceira ordem e seus respectivos resíduos. Adaptado da referência [50].

Tabela 3. Síntese dos diferentes procedimentos adotados para a obtenção do espectro de A2F incluindo as respectivas atribuições dos valores de $\Gamma$, das equações utilizadas para obter os espectros e das contribuições presentes em cada procedimento. ${ }^{*} \mathrm{G}_{\mathrm{R}}-$ Geometria rígida, $\mathrm{G}_{\mathrm{F}}-$ Geometria flexível.

Tabela 4. Momento de dipolo do estado fundamental (em Debye). * Resultados obtidos aproximando as moléculas de solvente como cargas pontuais.

Tabela 5. Seção de choque de A2F da pNA em solução. Os valores das energias de transição referentes a intensidade máxima de absorção $(\omega \max )$ e das larguras de linha $\Gamma$ estão em eV e os valores das seções de choque de $\mathrm{A} 2 \mathrm{~F}(\sigma)$ estão em GM. Os resultados experimentais (EXP) foram extraídos das referências [112,127]. Os valores ajustados de $\Gamma f i t$ utilizados em P5 estão apresentados entre parênteses.

Tabela 6. Energia de transição relacionada ao máximo valor da seção de choque de A2F ( $\omega$ max) em $\mathrm{eV}$, largura de linha total $\Gamma$ tot em eV e seção de choque de A2F $(\sigma)$ em GM obtidos de simulações BOMD

Tabela 7. Variação das propriedades eletrônicas relacionadas com o processo de A1F e de A2F da pBT considerando a aproximação PCM. *Entre parênteses apresentamos resultados experimentais [157], ins = insolúvel.

Tabela 8. Energia de transição e momento de dipolo permanente e de transição para a pBT em diferentes aproximações de solventes. $\Delta \mu=\mu g g-\mu e e$. ASEC-n: $\mathrm{n}$ representa o número da iteração no processo de polarização utilizando simulações moleculares, para $n=0$ as cargas utilizadas foram obtidas com a aproximação PCM. *A geometria utilizada nas simulações é a mesma que a utilizada no cálculo com PCM.

Tabela 9. Valores máximos dos espectros de A1F. Resultados obtidos com conjunto de funções base 6-31+G(d) para cálculos utilizando diferentes funcionais DFT. Os resultados para os compostos reduzidos estão entre parênteses. ${ }^{a}$ Os espaços ativos selecionados foram: 7 - OC=31(31) e $\mathrm{NO}=40(34) ; 10-\mathrm{OC}=31(34)$ e $\mathrm{NO}=43(38)$ para compostos completos (reduzidos).

Tabela 10. Energia do máximo de absorção da banda de baixa ( $\Delta E 1)$ e de alta $(\Delta E 2)$ energia, $\Delta E$ em $\mathrm{eV}$, e intensidade da seção de choque de $\mathrm{A} 2 \mathrm{~F}(\sigma-\mathrm{em} \mathrm{GM})$. Resultados dos compostos reduzidos entre parênteses e considerando a aproximação PCM para o solvente água em itálico. 
Tabela 11. Valores EFISHG e HRS obtidos para perturbações dinâmicas com 1907 nm em função do método de otimização de geometria e da posição do ânion. Os valores reportados estão nas unidades $\mu \beta / /$ (10ª.u.), $\beta / /$ (a.u.), $\mu$ (a.u.), $\theta(\mu, \beta)$ (deg) é o ângulo entre os vetores $\mu$ e $\beta, \beta H R S$ (a.u.)... 104 Tabela 12. Valores das respostas EFISHG para perturbações dinâmicas com $1907 \mathrm{~nm}$ em função do método de otimização de geometria e da posição do ânion. Os valores reportados estão na unidades: $\mu \beta / / k T, \gamma / /$ e $\gamma E F I S H G\left(10^{4} \mathrm{au}\right) ; \mu \beta / /$ eff e $\mu \beta / / \exp \left(10^{-48} \mathrm{esu}\right), R 32=3 k T \times \gamma / / \mu \beta / /$. $\mathrm{T}=298.15 \mathrm{~K}, 3 \mathrm{kT}=2.833 \times 10-3$ u.a

Tabela 13. Valores médios e desvio padrão da BLA $(\AA)$ e da distância $d_{\mathrm{NA}}(\AA)$ obtidos das DM. Os valores da BLA das geometrias DFT de mínima energia obtidas estão entre parênteses 106

Tabela 14. Comprimentos de onda (nm) associados com os máximos das absorções. As intensidades teóricas estão normalizadas em referência ao complexo 1 que apresenta o valor de 1.38 para a intensidade máxima. 107

Tabela 15. Valores médios e desvios padrões para as respostas EFISHG e HRS obtidas em $1907 \mathrm{~nm}$.

Tabela 16. Valores médios e desvios padrões das respostas EFISHG obtidas em $1907 \mathrm{~nm} . R 3 / 2=$ $(3 k T \times \gamma / /) / \mu \beta / /$ onde $\mathrm{T}=298.15 \mathrm{~K}, 3 k T=2.833 \times 10^{-3}$ u.a. 


\section{LiSTA DE SIGlaS E ACRônIMOS}

2LM

A1F

$\mathrm{A} 2 \mathrm{~F}$

ASEC

B3LYP

B88

BHandHLYP

BLYP-D3

BOMD

CAM-B3LYP

CHELPG

CPKS

D3

DFT

DM

DMSO

DR

EFISHG

$\mathrm{G}_{\mathrm{F}} / \mathrm{wF}$

GM

$\mathrm{G}_{\mathrm{R}} / \mathrm{nF}$

$\mathrm{G}_{\mathrm{R}} / \mathrm{wF}$

$\mathrm{HF}$

HK

HOMO
Two-level model

Absorção de um fóton

Absorção de dois fótons

Average Solvent Electrostatic Configuration

Funcional DFT

Funcional DFT

Funcional DFT

Funcional DFT com termo de dispersão de Grimme

Born-Oppenheimer Molecular Dynamic

Funcional DFT com correções de longo alcance para o funcional B3LYP

Charges from Electrostatic Potentials using a Grid

Coupled Perturbed Kohn-Sham

Termo de dispersão de Grimme utilizado em conjunto com funcionais DFT

Density Functional Theory

Dinâmica molecular

Dimetilsulfóxido

Taxa de despolarização

Electric Field-Induced Second Harmonic Generation

Geometria e propriedades eletrônicas obtidas na presença de campo elétrico $\mathrm{F}$

Unidade da seção de choque de A2F. 1 u.a. é igual a 1.896788 GM e 1 GM representa $10^{-50} \mathrm{~cm}^{4} \mathrm{~s} /$ photon

Geometria e propriedades eletrônicas obtidas na condição de molécula isolada

Geometria obtida na condição de molécula isolada e propriedades eletrônicas obtidas na presença de campo elétrico $\mathrm{F}$

Hartree-Fock

Hohemberg e Kohn

Highest Occupied Molecular Orbital 


\begin{tabular}{|c|c|}
\hline HRS & Hyper-Rayleigh Scattering \\
\hline INDO & Intermediate Neglect Differential Overlap \\
\hline $\mathrm{KS}$ & Kohn e Sham \\
\hline LSDA & Funcional DFT \\
\hline LUMO & Lowest Unoccupied Molecular Orbital \\
\hline $\mathrm{MC}$ & Monte Carlo \\
\hline MM & Molecular Mechanics \\
\hline MP2 & Teoria de Perturbação de Møller-Plesset de segunda ordem \\
\hline MUSPEC & Multiconfigurational Spectrum Convolution \\
\hline NPT & Ensemble com número de partículas, pressão e temperatura constantes \\
\hline NVT & Ensemble com número de partículas, volume e temperatura constantes \\
\hline oBT & ortho-Betaína \\
\hline ONL & Óptica Não Linear \\
\hline OPLS-AA & Optimized Potentials for Liquid Simulations - All Atom \\
\hline pBT & para-Betaína \\
\hline PCM & Polarizable Continuum Model \\
\hline pNA & Para-Nitroanilina \\
\hline pós-HF & pós-Hartree-Fock \\
\hline $\mathrm{QM} / \mathrm{MM}$ & Quantum Mechanics / Molecular Mechanics \\
\hline QR & Teoria de resposta quadrática \\
\hline RG & Runge e Gross \\
\hline SCF & Self Consistent Field \\
\hline SHG & Second Harmonic Generation \\
\hline $\mathrm{SPC} / \mathrm{E}$ & Campo de força para água \\
\hline S-QM/MM & Sequential-Quantum Mechanics / Molecular Mechanics \\
\hline TC & Transferência de carga \\
\hline TD-DFT & Time-Dependent Density Functional Theory \\
\hline ZINDO & INDO com parametrização espectroscópica realizado por M. Zerner \\
\hline ZINDO/S & Cálculos realizados com ZINDO considerando excitações simples \\
\hline ZINDO/SD & Cálculos realizados com ZINDO considerando excitações simples e duplas \\
\hline
\end{tabular}




\section{SUMÁRIO}

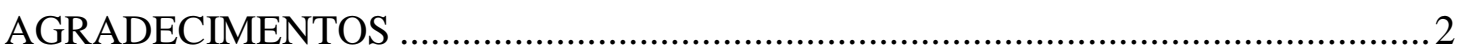

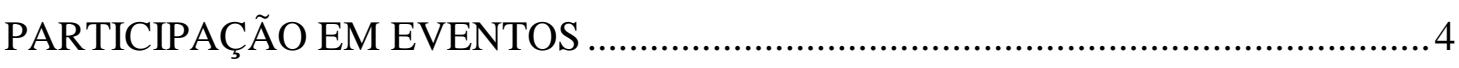

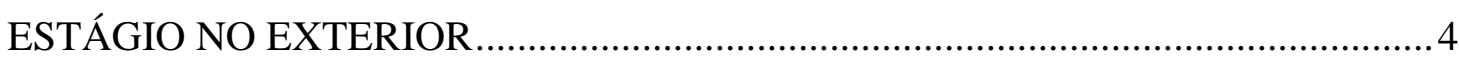

PRODUÇÃO DURANTE O DOUTORADO .........................................................

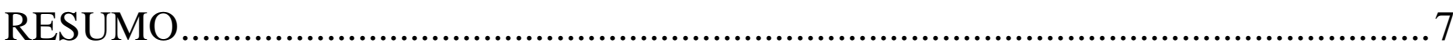

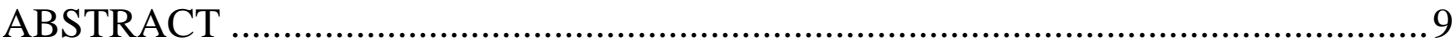

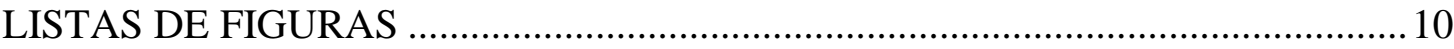

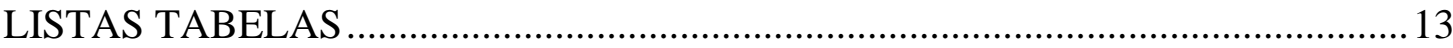

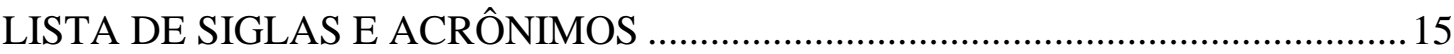

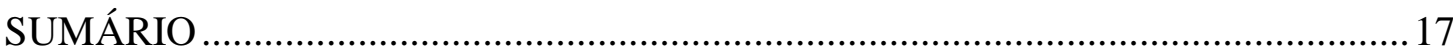

CAPÍTULO 1 INTRODUÇÃO ....................................................................... 19

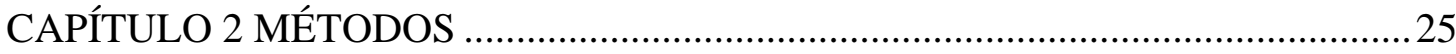

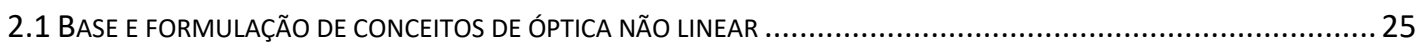

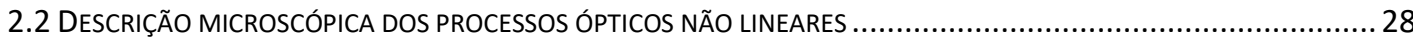

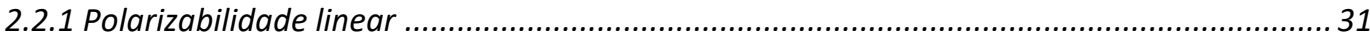

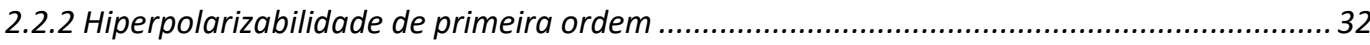

2.2.3 Hiperpolarizabilidade de segunda ordem ....................................................................... 33

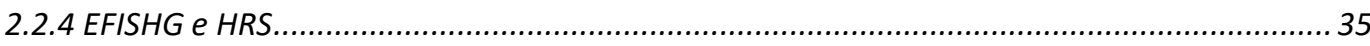

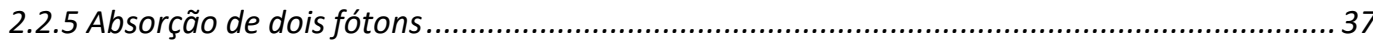

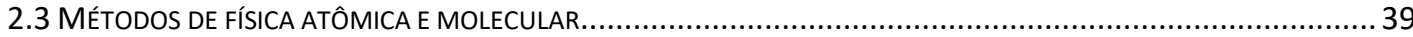

2.3.1 Teoria do funcional da densidade ............................................................................... 41

2.3.2 Teoria do funcional da densidade dependente do tempo................................................. 43

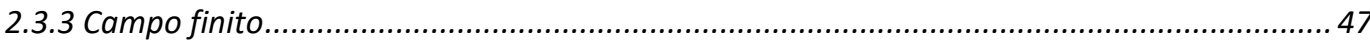

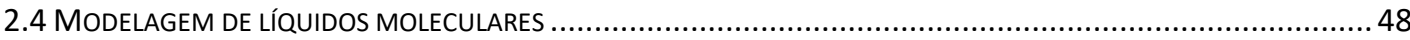

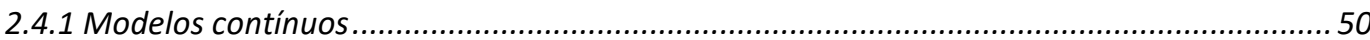

2.4.2 Amostragem de Monte Carlo Metropolis ........................................................................ 51

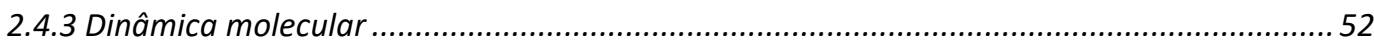

2.4.4 Metodologia Sequential - Quantum Mechanics / Molecular Mechanics ........................... 55 


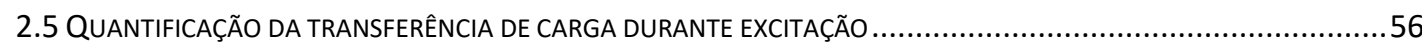

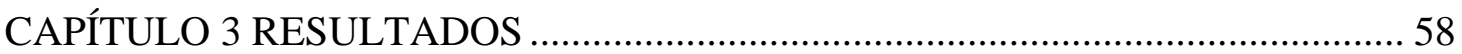

3.1 ALARGAMENTO DE LINHA ESPECTRAL PARA SIMULAÇÃO DA SEÇÃO DE CHOQUE DE ABSORÇÃO DE DOIS FÓTONS DA

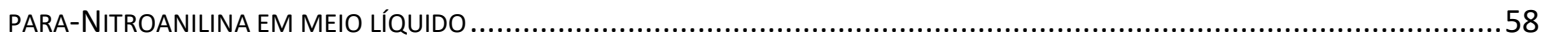

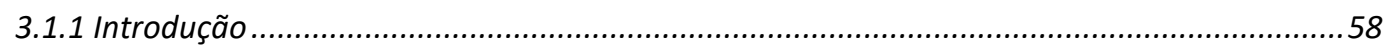

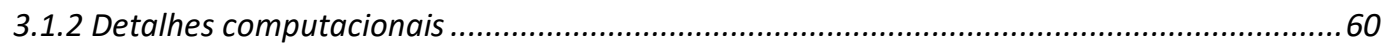

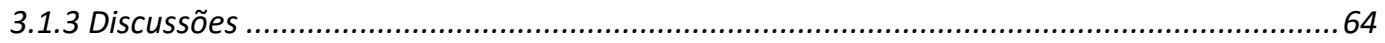

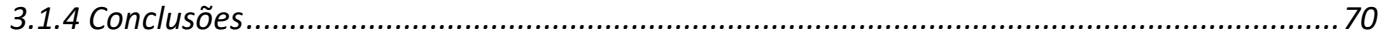

3.2 EFEITOS ESTRUTURAIS E DE SOLVENTE NA DESCRIÇÃO DA ABSORÇÃO DE DOIS FÓTONS DE PIRIDÍNIO N-FENOLATO .. 72

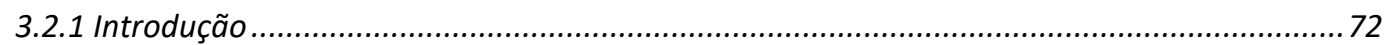

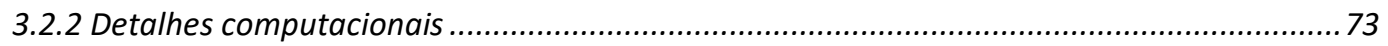

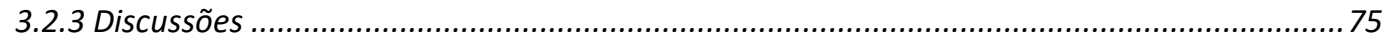

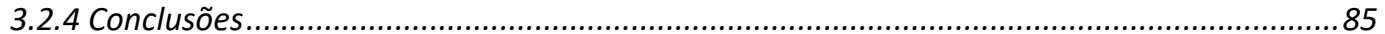

3.3 ESPECTRO DE ABSORÇÃO DE DOIS FÓTONS DE SISTEMAS MOLECULARES GRANDES.......................................86

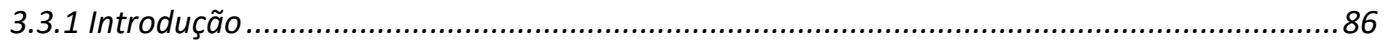

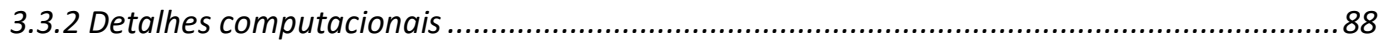

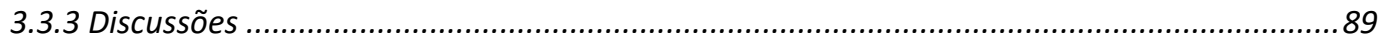

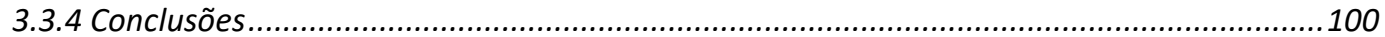

3.4 DESVENDANDO A RESPOSTA EFISHG DE COMPLEXOS DE STILBAZOLIUM FORMADOS POR PARES IÔNICOS EM

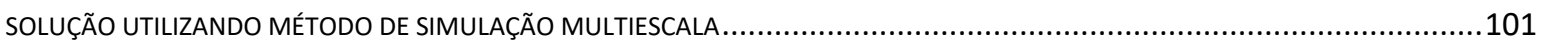

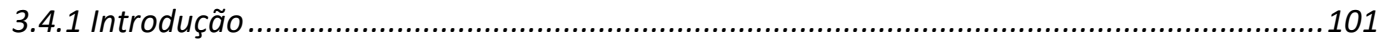

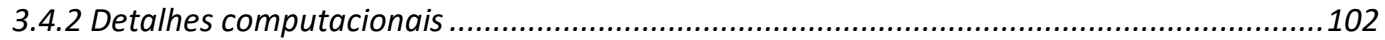

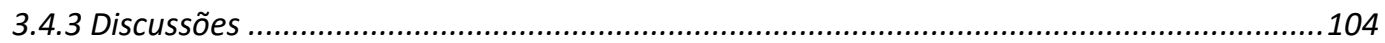

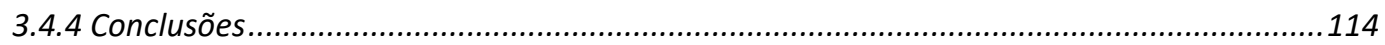

CAPÍTULO 4 CONCLUSÕES E PERSPECTIVAS ................................................ 115

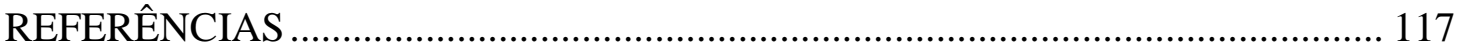

ANEXO A. MULTICONFIGURATIONAL SPECTRUM CONVOLUTION ...... 129

ANEXO B. ARTIGOS PUBLICADOS ............................................................... 134 


\section{Capítulo 1 INTRODUÇÃo}

A interação da radiação eletromagnética com a matéria é tema de grande interesse desde os primórdios da humanidade, englobando desde fenômenos presentes em nosso cotidiano como reflexão e refração da luz até o desenvolvimento de formas de energia limpa como células fotovoltaicas. Essas interações radiação-matéria ocorrem em ampla faixa do espectro eletromagnético, sendo responsáveis por muitos fenômenos ópticos. Em escala microscópica, as excitações vibracionais e eletrônicas ocorrem devido às interações moleculares com a radiação de energia equivalente àquela da região entre o infravermelho e o ultravioleta do espectro eletromagnético.

Do ponto de vista microscópico as interações da luz com a matéria ocorrem entre moléculas e fótons. Dessas interações resultam diversos fenômenos como: excitações vibracionais - quando a energia do fóton é convertida em energia térmica; espalhamento elástico - quando o fóton muda de direção ao interagir com a molécula; efeito fotoelétricos quando a energia do fóton é utilizada para remover um elétron da matéria; entre outros fenômenos. Compreender essas interações permite manipular esses fenômenos e consequentemente desenvolver tecnologias.

O processo de absorção de um fóton (A1F) com energia $\omega$, adotando $\hbar=1$, conduz um sistema atômico/molecular de um estado inicial, com energia $E_{0}$, para um estado final após uma excitação eletrônica, onde a energia do sistema molecular é $E_{0}+\omega$. Por outro lado, no processo de absorção multifotônico envolvendo $n$ fótons o sistema visita $n$-1 estados intermediários, também chamados estados virtuais, antes de acessar um estado final. A absorção de dois fótons (A2F) é um processo óptico onde dois fótons, com energias $\omega_{1}$ e $\omega_{2}$, são simultaneamente absorvidos pelo sistema atômico/molecular levando-o a um estado final energeticamente excitado. Neste processo a energia dos fótons absorvidos é responsável pela excitação de um elétron, onde a energia do sistema é $E_{0}+\omega_{1}+\omega_{2}$. Uma ilustração dos processos de A1F e A2F está apresentada na Figura 1. O processo de A2F foi pioneiramente previsto por GöppertMayer [1,2] em 1931 e está relacionado com a parte imaginária da hiperpolarizabilidade de segunda ordem (ou susceptibilidade elétrica de terceira ordem). Devido ao fato da intensidade 
da A2F variar com o quadrado da intensidade da radiação incidente o processo de A2F foi confirmado experimentalmente 30 anos depois por Kaiser e Garrett [3] após o advento de sistemas lasers. Devido à dependência quadrática com a intensidade da radiação incidente a A2F é um processo óptico não linear (ONL).

A1F

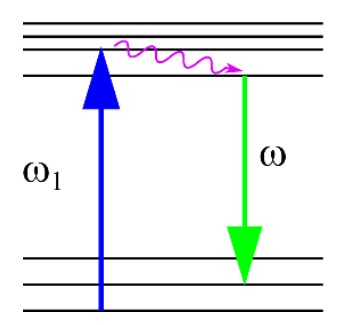

$\mathrm{A} 2 \mathrm{~F}$

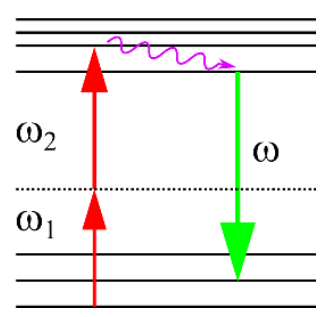

Figura 1. Diagrama de absorção de um fóton de energia $\omega_{1}$ e de dois fótons de energias $\omega_{1}$ e $\omega_{2}$.

Atualmente, com a disponibilidade de lasers de maior intensidade e pulsos de menor duração temporal é possível evitar efeitos térmicos e desenvolver tecnologias que exploram tal processo óptico não linear em diversas áreas do conhecimento, tais como: armazenamento de dados em estruturas tridimensionais [4-6], microscopia por fluorescência e imagiamento in vivo [7-9], microfabricação [10], terapia fotodinâmica [11,12], limitação óptica [13] e conversão de luz laser (up-conversion) [14]. Um aspecto preliminar e muito importante para o contínuo desenvolvimento de aplicações tecnológicas é a caracterização das propriedades ONL de novos compostos, buscando compreender os mecanismos envolvidos nesses processos e desenvolvendo compostos com maior taxa de A2F [15-17]. Algumas dessas aplicações estão ilustradas na Figura 2. A exemplo disso, hoje sabe-se que a elevada resposta óptica não linear de um sistema está com frequência relacionada com o grau/nível da transferência de carga (TC) promovida durante a excitação [18].

As transições eletrônicas resultantes dos processos de A1F e de A2F em moléculas centrossimétricas possuem caráter espectroscópicos distintos, mas complementares, de tal forma que transições proibidas por A1F são permitidas por A2F e vice-versa. Tal característica contribui na descrição da estrutura eletrônica dessa categoria de moléculas. Isso é possível devido às regras de seleção, relacionadas com as simetrias dos orbitais, desses processos serem diferentes [19]. Para que um processo de A2F puro ocorra é importante que as energias dos fótons incidentes estejam longe de possíveis ressonâncias com estados eletrônicos moleculares, evitando assim a manifestação da A1F e subsequentes excitações entre estados excitados [20].

Outro fenômeno ONL é a geração do segundo harmônico (Second Harmonic Generation - SHG), o qual pode ser utilizado para duplicar a frequência da luz laser. Devido à 
sensibilidade à simetria molecular, utilizando a técnica SHG é possível realizar bioimagem [21] ou projetar dispositivos lógicos de escala molecular [22]. A técnica da geração de segundo harmônico induzida por campo elétrico (Electric Field-Induced Second Harmonic Generation - EFISHG) já é bem estabelecida e muito utilizada na determinação da primeira e a segunda hiperpolarizabilidades de compostos em soluções.

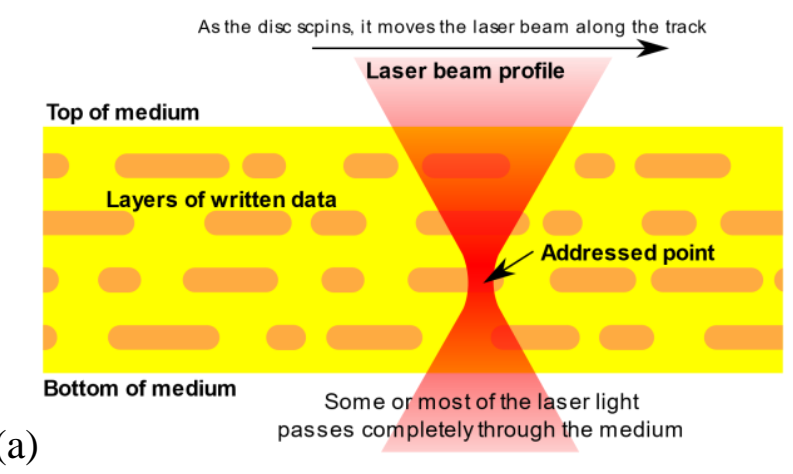

\section{1-photon vs. 2-photon}

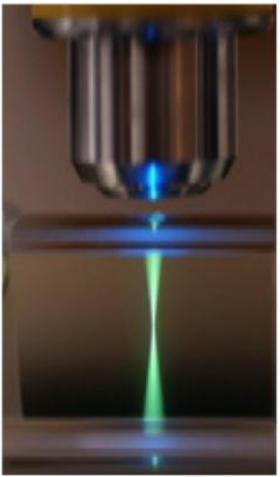

Fluorescence from (c)

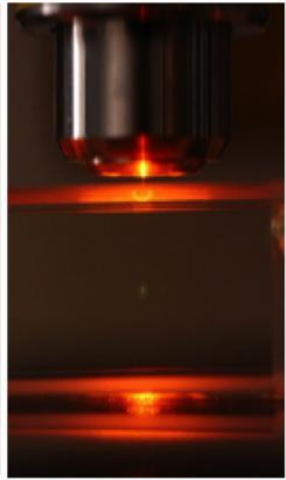

Fluorescence from focal spot only
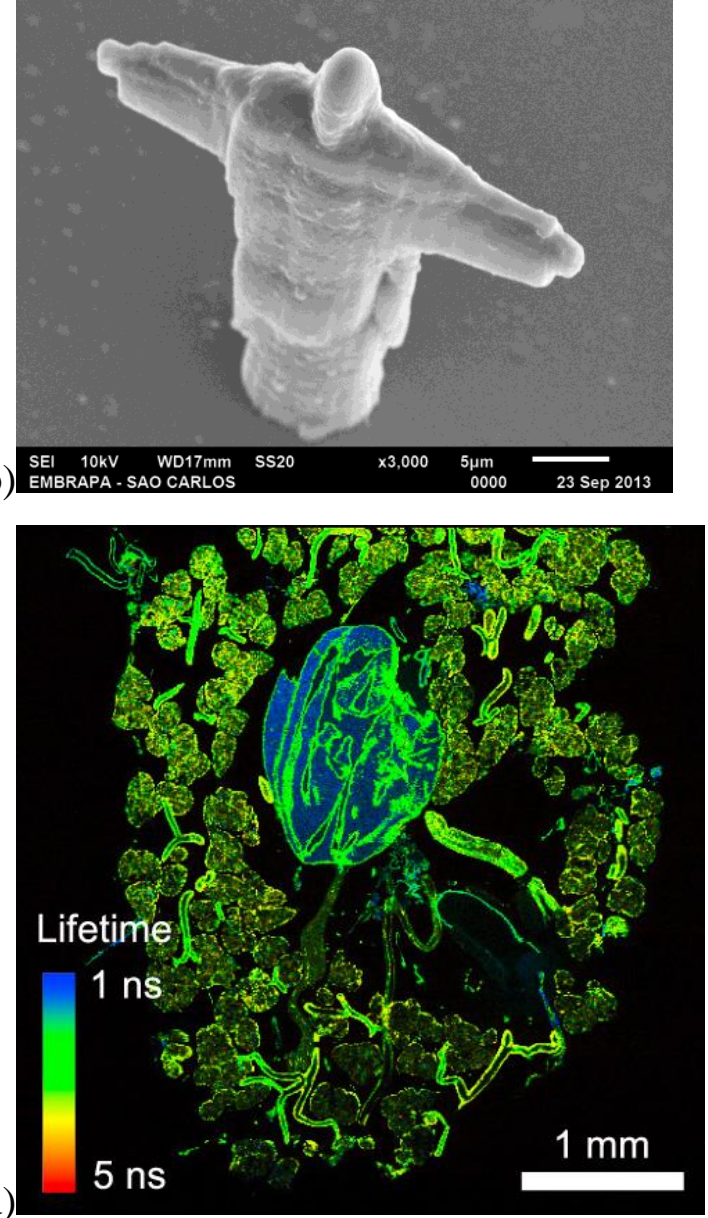

Figura 2. (a) Representação de um disco óptico de armazenamento tridimensional (em amarelo) e das trilhas de gravação (em laranja) [23]. (b) Microfabricação do Cristo Redentor em material polimérico [24]. (c) Resolução da fluorescência promovida pelos processos de absorção de um e de dois fótons [25]. (d) bioimagiamento da concentração de cloreto em glândulas salivares [26].

A técnica EFISHG requer a aplicação de um campo eletrostático externo para criar uma orientação preferencial, portanto não pode ser utilizada para investigar compostos carregados devido à indefinição do momento dipolo molecular [27]. No entanto, além de muitas aplicações para moléculas neutras, a técnica EFISHG tem sido utilizada recentemente para determinar as respostas ONL de complexos neutros constituídos por pares ânion-cátion. Tais complexos requerem o uso de solventes com baixos valores de constante dielétrica para favorecer a associação dos íons e evitar a dissociação. Esta estratégia de engenharia molecular foi demonstrada primeiramente no ano de 2000 para moléculas amphiphilic polyenic push-pull [28] 
e aplicada no ano de 2014 a chaves moleculares (do termo inglês molecular switches) acionadas por variações de pH [29]. Embora algumas medidas utilizando a técnica EFISHG já tenham sido relatadas para pares de íons, pouco tem sido feito para sua modelagem usando métodos de química teórica [30]. Portanto, utilizar simulações computacionais da técnica EFISHG em pares iônicos é um campo de pesquisa em aberto.

Dados experimentais e resultados teóricos evidenciam que em sistemas moleculares orgânicos as interações com o meio solvente podem desempenhar importante papel sobre as propriedades da A2F [31-33]. Contudo, grande parte desses estudos teóricos realizados para descrever os processos ópticos lineares e não lineares em solução incluem os efeitos de solvente no cálculo da estrutura eletrônica de forma implícita [34-38]. Algumas abordagens mais recentes propõem a utilização de metodologias multiescala combinando métodos de mecânica quântica e mecânica molecular (Quantum Mechanics / Molecular Mechanics - QM/MM) para estudar os efeitos de solvente sobre a A1F [39]. Mais recentemente, os métodos QM/MM passaram a ser aplicados no estudo de processos ONL de moléculas orgânicas e permitiram obter descrições mais precisas de tais processos em meio líquido [30,40-47]. Apresentamos mais detalhes sobre a inclusão dos efeitos de solventes na estrutura eletrônica de moléculas na seção 2.4 - Modelagem de líquidos moleculares.

Dentre os diversos processos de interação da radiação com a matéria, neste trabalho serão abordados processos ONLs relacionados com os termos de segunda e terceira ordens, dando ênfase ao processo de A2F. Para incluir os efeitos de solventes nas propriedades eletrônicas consideramos as aproximações Born-Oppenheimer Molecular Dynamics (BOMD), Dinâmica Molecular Clássica (DM), amostragem de Monte Carlo Metropolis (MC), Polarizable Continuum Model (PCM) e campos eletrostáticos. O objetivo do trabalho foi descrever de forma precisa os mecanismos envolvidos no processo de A2F buscando melhores descrições da seção de choque de A2F de cromóforos em meio solvente. Além disso, estratégias alternativas para aumentar a intensidade das transições permitidas por A2F e abordagens para a realização de estudos da $\mathrm{A} 2 \mathrm{~F}$ em moléculas com grande quantidade de átomos foram também exploradas.

Este texto está organizado da seguinte maneira: inicialmente introduzimos os conceitos e as formulações da óptica linear e não linear seguido de uma descrição relacionada às propriedades de A2F e a resposta EFISHG de moléculas orgânicas. Em seguida abordaremos alguns aspectos da física atômica e molecular segundo a formulação da mecânica quântica e finalmente encerramos o capítulo de métodos com a descrição de alguns modelos de líquidos 
moleculares. Seguindo, serão apresentadas as seções dos estudos realizados. Cada seção terá sua própria contextualização, detalhes computacionais, apresentação de resultados, discussões e conclusões. Finalmente, as conclusões e perspectivas do trabalho são apresentados no último capítulo. Salientamos que este texto utiliza a convenção americana da separação de números decimais.

O primeiro estudo apresentado no capítulo de resultados aborda diferentes formas de considerar os efeitos de solventes na descrição da seção de choque de A2F da molécula paraNitroanilina. Apresentamos uma discussão sobre como atribuir o valor do parâmetro relacionado com o alargamento espectral. As interações do soluto com os solventes foram incluídas de forma explícita utilizado simulações BOMD e MC e de forma implícita com a aproximação PCM. Mostramos que a inclusão de moléculas explicitas dos solventes é essencial para descrever a seção de choque de A2F. Além disso, para construir o espectro de absorção devemos atribuir um valor de largura de linha relacionado com o alargamento espectral, sendo que geralmente o valor desse parâmetro é atribuído de forma arbitrária. Dentre as possíveis e já bem conhecidas formas de se obter um valor de largura de linha para ser usado, os resultados obtidos apresentaram tendências distintas para o valor da seção de choque de A2F nos diferentes solventes considerados. Assim, propomos uma forma de ajustar a largura do espectro teórico com o espectro experimental. Neste procedimento de ajuste, centenas de configurações extraídas de simulações moleculares são consideradas para construir o espectro de absorção médio, de tal modo que a largura da banda deste espectro médio seja igual ao valor da largura de linha experimental. Os resultados obtidos com este ajuste forneceram as melhores descrições da seção de choque de A2F. Mostramos ainda que o valor obtido através desse ajuste reproduz bem a contribuição homogênea do alargamento espectral para simulações realizadas com geometrias rígidas. Portanto, além de obter boa descrição da seção de choque de A2F, o procedimento nos possibilitou atribuir o valor da largura de linha de forma não arbitrária.

Seguindo, no segundo estudo analisamos os valores das seções de choque de A2F das moléculas para-betaína e ortho-betaína considerando: variações estruturais devido a torções induzidas; variações da amplitude de um campo eletrostático externo; e considerando a interação soluto-solvente com simulações de MC e com PCM. Os resultados da seção de choque de A2F obtidos utilizando o modelo simplificado de dois níveis apresentaram boa concordância com os resultados obtidos utilizando o método baseado na teoria de resposta quadrática, embora seja evidenciado no caso da ortho-betaína a existência de contribuições destrutivas de outros estados reduzindo a seção de choque de A2F. Mostramos ainda que é possível utilizar campos 
elétricos externos para modular a intensidade da $\mathrm{A} 2 \mathrm{~F}$ ao mesmo tempo que o processo de $\mathrm{A} 1 \mathrm{~F}$ é inibido. Este resultado pode ser utilizado para aumentar a intensidade da A2F e, ao mesmo tempo, evitar os efeitos de engrandecimento devido ao processo de A1F.

Na seção seguinte, apresentamos o estudo teórico dos espectros de absorção linear e não linear de dois compostos sintetizados com elevado potencial para bioimagiamento por fluorescência por A2F [48]. Esses compostos são constituídos por mais de 200 átomos e são um desafio para cálculos de A2F utilizando métodos de química computacional mais acurados. Portanto, confrontar os resultados obtidos com métodos de baixo custo computacional e aqueles oriundos de métodos menos aproximados é de grande relevância para a modelagem teórica dessa categoria de moléculas. A utilização de métodos semi-empíricos considerando excitações simples e duplas resultou em uma descrição mais precisa das seções de choque de A2F comparativamente com cálculos mais rigorosos baseados em teoria de resposta quadrática. Portanto, o estudo realizado evidencia o excelente custo benefício atingido ao utilizar métodos semi-empíricos para a obtenção da seção de choque de A2F de moléculas com tamanhos hoje ainda inacessíveis a outros métodos computacionalmente mais custosos.

A última seção do capítulo de resultados expõe o trabalho realizado em período de estágio no exterior onde as respostas não lineares de segunda e terceira ordem de diversos complexos compostos por pares ânion-cátion foram avaliadas. Utilizando DM, mostramos para pares iônicos em meio líquido que a resposta EFISHG é modulada pela posição relativa entre os íons. Esta modulação é controlada pela variação da direção do momento de dipolo causado pela distribuição dos sítios atômicos carregados e não por variações das componentes da primeira hiperpolarizabilidade.

Durante o período de doutorado iniciamos o desenvolvimento de um software para obter o espectro de A2F utilizando contribuições de diversas configurações. No Anexo A apresentamos brevemente o programa Multiconfigurational Spectrum Convolution MUSPEC. A principal utilidade deste programa é a obtenção e análise de espectros de absorção linear e não linear para conjuntos de configurações extraídas de simulações moleculares de forma interativa e visual. Tais análises podem ser facilmente estendidas para outros estudos que envolva a convolução de funções Gaussianas ou Lorentzianas desde que fornecidos as respectivas energias e intensidades necessárias para a convolução das funções de alargamento. 


\section{Capítulo 2 MÉTODOS}

Neste capítulo abordamos os conceitos e as formulações teóricas necessárias para o desenvolvimento dos estudos sobre óptica não linear de moléculas orgânicas em solução realizados neste trabalho. As interações da luz com a matéria serão analisadas em processos ONLs de segunda e de terceira ordem, relacionando teoria e experimento. Uma breve introdução sobre as técnicas de física computacional utilizadas para a obtenção da estrutura eletrônica e diferentes formas de aproximação para descrever as interações soluto-solvente complementam esse capítulo. Para estudos mais profundos aconselhamos a leitura das referências citadas no decorrer do texto.

\subsection{Base e formulação de conceitos de óptica não linear}

A matéria é constantemente irradiada por ondas eletromagnéticas proveniente de fontes naturais, como o sol, ou de fontes artificiais, como antenas de televisão. Dessas interações, observamos os fenômenos de reflexão e refração dentro do espectro visível e diversos outros processos “invisíveis" como a transmissão/recepção de sinais de rádio, de televisão e recentemente de internet. Geralmente, a intensidade desses processos varia linearmente com a intensidade da radiação e são considerados processos ópticos lineares. Por outro lado, fenômenos ópticos cujas intensidades variam com maiores potências da intensidade da radiação (como com o quadrado da intensidade) são classificados como processos ópticos não lineares e deram origem à área de pesquisa denomina Óptica Não Linear (ONL). Para mais detalhes sobre processos ópticos lineares e não lineares, assim como sobre as metodologias e técnicas experimentais mais empregadas na área recomendamos a leitura dos livros [19,49-53] e dos artigos científicos [11,54-59].

Essencialmente, os efeitos ONLs são evidentes quando o campo elétrico da radiação e o campo elétrico interatômico são comparáveis. Para isso, geralmente, é necessário irradiar o material com um feixe de intensidade da ordem $100 \mathrm{GW} / \mathrm{cm}^{2}$. Contudo, devido às ressonâncias da energia do fóton incidente com os níveis de energia eletrônico do composto, os efeitos ONL podem ser observados mesmo para radiações de menor intensidade. 
$\mathrm{Na}$ escala macroscópica, quando a matéria é irradiada por um feixe de luz descrito por um campo externo $\boldsymbol{E}(t)$, os processos de absorção podem ser analisados pela taxa de energia absorvida do feixe por unidade de volume do meio

$$
\left\langle\frac{d}{d t}\left(\frac{\text { Energia absorvida }}{\text { Volume }}\right)\right\rangle_{\text {tempo }}=\langle\boldsymbol{j} \cdot \boldsymbol{E}\rangle
$$

Para sistemas não magnéticos onde não há cargas nem correntes livres, a densidade de corrente $(\boldsymbol{j})$ induzida no meio pode ser expressa por

$$
\boldsymbol{j}=\frac{\partial \boldsymbol{P}}{\partial t}+c \nabla \times \boldsymbol{M}-\frac{\partial}{\partial t} \nabla \times \boldsymbol{Q}+\cdots
$$

onde os termos da expansão multipolar estão relacionados, com a polarização do dipolo elétrico $\partial \boldsymbol{P} / \partial t$, dipolo magnético $c \nabla \times \boldsymbol{M}$ e quadrupolo elétrico $\partial / \partial t(\nabla \times \boldsymbol{Q})$. As propriedades ou processos ONL abordados neste trabalho são, em geral, predominantemente relacionados aos termos do dipolo elétrico, por esta razão os demais termos da expansão serão desconsiderados.

No regime de altas intensidades, a polarização elétrica induzida $\boldsymbol{P}(t)$ pode ser descrita por uma série de potências em função do campo elétrico aplicado $\boldsymbol{E}(t)$ da radiação

$$
\begin{aligned}
\boldsymbol{P}(t) & =\chi^{(1)} \boldsymbol{E}(t)+\chi^{(2)} \boldsymbol{E}^{2}(t)+\chi^{(3)} \boldsymbol{E}^{3}(t)+\cdots \\
& =\boldsymbol{P}^{(1)}(t)+\boldsymbol{P}^{(2)}(t)+\boldsymbol{P}^{(3)}(t)+\cdots
\end{aligned}
$$

As quantidades macroscópicas $\chi^{(n)}$ são conhecidas como susceptibilidades elétricas de $n$-ésima ordem, sendo elas tensores de grau $n+1$, e $\boldsymbol{P}^{(n)}(t)$ são os correspondentes termos da expansão da polarização elétrica

Os processos de absorção óptica estão relacionados com os termos de polarização da expansão, apresentada na equação (3), que apresentam contribuições na mesma frequência do campo incidente. Os termos com frequências múltiplas da frequência do campo incidente estão associados com a geração de harmônicos. Dessa forma, para radiações oscilantes, somente os termos de polarização ímpares dessa expansão contribuem nos processos de absorção óptica. Com propósito demonstrativo considere a aplicação de um campo monocromático $\boldsymbol{E}(t)=$ $E \cos (\omega t)$.

$$
\begin{aligned}
& \boldsymbol{P}^{(1)}(t)=\chi^{(1)} E \cos (\omega t) \\
& \boldsymbol{P}^{(2)}(t)=\chi^{(2)}(E \cos (\omega t))^{2}=\frac{\chi^{(2)} E^{2}(1+\cos (2 \omega t))}{2}
\end{aligned}
$$




$$
\boldsymbol{P}^{(3)}(t)=\chi^{(3)}(E \cos (\omega t))^{3}=\frac{\chi^{(3)} E^{3}(3 \cos (\omega t)+\cos (3 \omega t))}{4}
$$

Combinando as equações (1), (2) e (3) é possível obter as expressões matemáticas que representam o processo de $\mathrm{A} 1 \mathrm{~F}$ e de $\mathrm{A} 2 \mathrm{~F}$ como apresentadas, respectivamente, pelas equações (5) e (6).

$$
\begin{aligned}
& \left\langle\frac{\partial \boldsymbol{P}^{(1)}(t)}{\partial t} \cdot \boldsymbol{E}(t)\right\rangle=2 \omega \operatorname{Im}\left[\chi^{(1)}\right] \boldsymbol{E}(t)^{2} \\
& \left\langle\frac{\partial \boldsymbol{P}^{(3)}(t)}{\partial t} \cdot \boldsymbol{E}(t)\right\rangle=6 \omega \operatorname{Im}\left[\chi^{(3)}\right] \boldsymbol{E}(t)^{4}
\end{aligned}
$$

Para obter as seções de choque de A2F associamos a variação da intensidade do feixe incidente com os coeficientes $a_{i}$ de absorção. Dessa forma, temos $a_{1}$ relacionado com A1F, $a_{2}$ com a A2F e assim sucessivamente. Sabendo que a intensidade da radiação é proporcional ao quadrado do campo elétrico podemos relacionar os coeficientes de absorção $a_{i}$ com a atenuação do feixe de luz ao atravessar o material

$$
\begin{aligned}
\frac{d I}{d z}=-\left(a_{1} I+a_{2} I^{2}+a_{3} I^{3}+\cdots\right) & \\
=- & {\left[\left(\frac{2 \pi \hbar \omega}{n c} \operatorname{Im}\left[\chi^{(1)}\right]\right) I+\left(\frac{24 \pi^{2} \hbar^{2} \omega}{n^{2} c^{2}} \operatorname{Im}\left[\chi^{(3)}\right]\right) I^{2}\right.} \\
& \left.+\left(\frac{160 \pi^{3} \hbar^{3} \omega}{n^{3} c^{3}} \operatorname{Im}\left[\chi^{(5)}\right]\right) I^{3}+\cdots\right]
\end{aligned}
$$

Do ponto de vista microscópico, podemos analisar os efeitos de campos elétricos externos na polarização molecular utilizando a variação do momento de dipolo induzido $\Delta \mu(t)$. Geralmente, $\Delta \mu(t)$ é expressa como uma expansão em série de Taylor em função do campo elétrico

$$
\Delta \mu(t)=\boldsymbol{\alpha} \boldsymbol{E}(t)+\frac{1}{2} \boldsymbol{\beta} \boldsymbol{E}^{2}(t)+\frac{1}{6} \boldsymbol{\gamma} \boldsymbol{E}^{3}(t)+\cdots
$$

onde $\boldsymbol{\alpha}$ é a polarizabilidade linear e $\boldsymbol{\beta}$ e $\boldsymbol{\gamma}$ são as hiperpolarizabilidades de primeira e segunda ordem respectivamente. A relação entre as grandezas macroscópicas $\left(\chi^{(n)}\right)$ e microscópicas $(\boldsymbol{\alpha}, \boldsymbol{\beta}, \boldsymbol{\gamma})$ podem ser obtidas comparando as equações (3) e (8). Da mesma forma como as susceptibilidades foram relacionadas com os processos de absorção, relacionamos os processos de A1F com $\boldsymbol{\alpha}$ e de A2F com $\boldsymbol{\gamma}$.

A estrutura eletrônica de sistemas atômicos e moleculares pode ser descrita utilizando estados estacionários obtidos a partir da solução da equação de Schrödinger. Estes estados 
estacionários também são chamados de estados reais ou de níveis de energia atômico/molecular. A excitação eletrônica destes estados ocorre quando o sistema atômico/molecular absorve fótons com energia localizada no espectro ultravioleta e visível. Além disso, para que o processo de A1F ocorra, a conservação de energia e de momento devem ser satisfeitas.

Quando a energia do fóton é menor que a necessária para excitar um elétron para outro nível de energia o sistema pode capturar o fóton formando um complexo composto pela molécula + fóton em um estado meta estável chamado de estado virtual. Esse estado virtual pode ser compreendido como um aprisionamento do fóton pela molécula de forma que não viole o princípio de incerteza. Enquanto o complexo está nesse estado virtual, outros fótons podem se incorporar ao sistema criando outros estados virtuais. O aprisionamento de múltiplos fótons neste estado metaestável pode resultar em (i) absorções multifotônicas - quando a soma da energia dos fótons absorvidos for igual a uma das energias de excitação para outro nível eletrônico, (ii) geração de harmônicos - através de mecanismos de espalhamento - quando a soma da energia dos fótons absorvidos for diferente das energias de excitação do sistema.

Embora alta densidade de fótons seja requerida para a manifestação de processos ONLs, nota-se que em processos multifotônicos a radiação incidente pode estar localizada na região infravermelha do espectro favorecendo diferentes aplicações. Na Figura 3 apresentamos quatro diagramas de energia, relacionados com: absorção de um fóton; espalhamento elástico; absorção de dois fótons; e a geração do segundo harmônico.

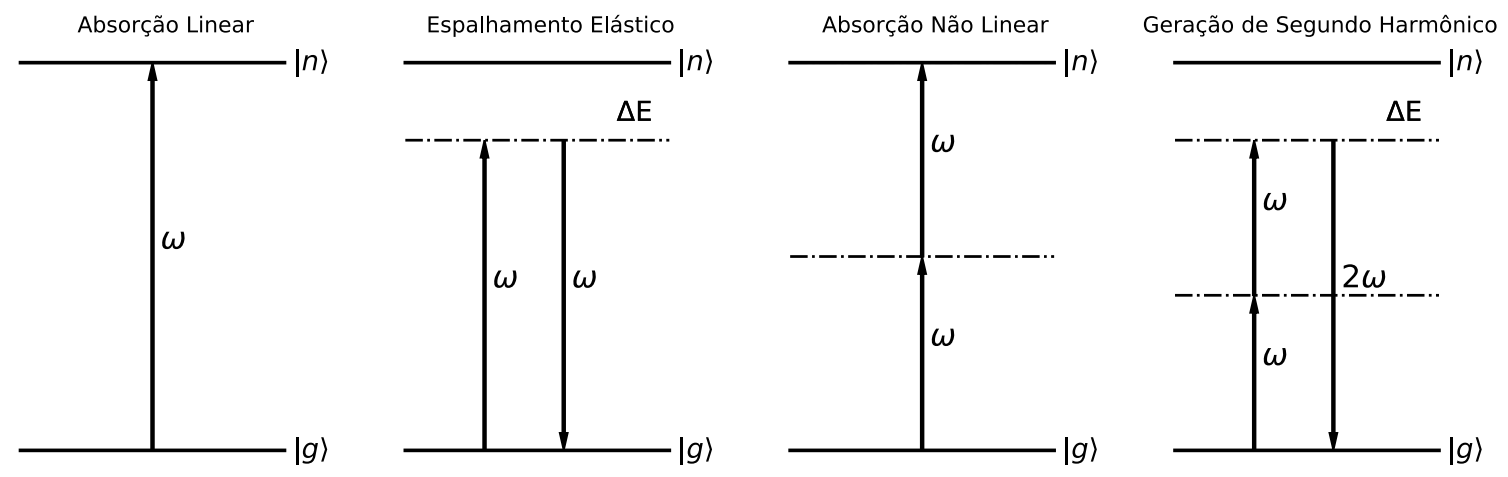

Figura 3. Representação diagramática dos níveis de energia molecular (linhas contínuas), dos estados virtuais (linhas tracejadas) e dos processos de absorção e emissão de fótons (setas). $\omega$ representa a energia do fóton e $\Delta E$ a diferença entre as energias do estado $|n\rangle$ e do estado virtual.

\subsection{Descrição microscópica dos processos ópticos não lineares}

Nesta seção apresentamos as expressões matemáticas que descrevem a interação da matéria com a radiação em nível microscópico utilizando a teoria de perturbação dependente 
do tempo. As soluções para as (hiper)polarizabilidades também são conhecidas como funções resposta. Nessa etapa assumimos conhecida a solução exata do sistema não perturbado. Para simplificar e padronizar a notação adotaremos o símbolo “^” para identificar operadores quânticos e omitiremos a dependência da posição.

A equação de Schrödinger dependente do tempo é a forma pela qual um vetor de estado $|\Psi(\mathrm{t})\rangle$ evolui temporalmente pela aplicação do operador Hamiltoniano $\widehat{H}(\mathrm{t})=\widehat{H_{0}}+\widehat{V}(t)$, onde $\widehat{H_{0}}$ é o Hamiltoniano do sistema não perturbado e $\widehat{V}(t)$ representa uma pequena perturbação no tempo.

$$
i \hbar \frac{\partial}{\partial t}|\Psi(\mathrm{t})\rangle=\widehat{H}(\mathrm{t})|\Psi(\mathrm{t})\rangle=\left(\widehat{H_{0}}+\widehat{V}(t)\right)|\Psi(t)\rangle .
$$

Representando $|\Psi(t)\rangle$ pela expansão do conjunto completo $\{|n\rangle\}$ de auto estados do operador $\widehat{H_{0}}$, com autovalores $E_{n}$, temos, agora, como objetivo encontrar os coeficientes $d_{n}(t)$ que satisfaçam

$$
|\Psi(t)\rangle=\sum_{n} d_{n}(t) e^{-i E_{n} t / \hbar}|n\rangle .
$$

Os coeficientes $d_{n}(t)$ representam pequenas perturbações temporais e são expandidos em ordens de grandeza, como apresentado na equação

$$
d_{n}(t)=d_{n}^{(0)}+d_{n}^{(1)}(t)+d_{n}^{(2)}(t)+d_{n}^{(3)}(t)+\cdots,
$$

Os valores dos coeficientes são obtidos de forma iterativa após a substituição da equação (11) na equação (10) seguida da aplicação dos operadores

$$
\left(i \hbar \frac{\partial}{\partial t}-\widehat{H}\right)\left\langle m\left|\mathrm{e}^{i E_{m} t / \hbar}\right| \Psi(t)\right\rangle
$$

A expressão matemática para os coeficientes é dada pela equação (13)

$$
i \hbar \frac{\partial}{\partial t} d_{m}^{(N)}(t)=\sum_{n}\langle m|\hat{V}(t)| n\rangle e^{i \omega_{m n} t} d_{n}^{(N-1)}(t),
$$

onde o sobrescrito (N) é a ordem da expansão do coeficiente e $\omega_{m n}=\left(E_{m}-E_{n}\right) / \hbar$ é a frequência relacionada com a energia de transição entre os estados $n$ e $m$. Para satisfazer a condição inicial define-se o coeficiente independente do tempo $d_{n}^{(0)}=\delta_{0 n}$.

Considerando a perturbação $\widehat{V}(t)=-\hat{\mu}_{\alpha} E_{\alpha}(t) e^{\epsilon t}$ (o fator $\epsilon$ é um infinitesimal positivo cuja função é garantir uma perturbação adiabática e nula para $t \rightarrow-\infty$ ) e o campo elétrico externo interagente $E_{\alpha}(t)=\sum_{\omega} E_{\alpha}^{\omega} e^{-i \omega t}$, representado pela combinação linear de campos 
monocromáticos oscilando em frequência $\omega$ no eixo molecular $\alpha^{1}$, definimos os coeficientes $d_{m}^{(N)}(t)$ como

$$
\begin{gathered}
d_{m}^{(1)}(t)=-\frac{1}{i \hbar} \int_{-\infty}^{t} \sum_{n, \omega_{1}}\left\langle m\left|\hat{\mu}_{\alpha} E_{\alpha}^{\omega_{1}} e^{-i \omega_{1} t^{\prime}} e^{\epsilon t^{\prime}}\right| n\right\rangle e^{i \omega_{m n} t^{\prime}} \delta_{0 n} d t^{\prime} \\
=\frac{1}{\hbar} \sum_{\omega_{1}} \frac{\left\langle m\left|\hat{\mu}_{\alpha}\right| 0\right\rangle E_{\alpha}^{\omega_{1}}}{\omega_{m 0}-\omega_{1}-i \epsilon} e^{i\left(\omega_{m 0}-\omega_{1}\right) t} e^{\epsilon t} \\
d_{m}^{(2)}(t)=-\frac{1}{i \hbar} \int_{-\infty}^{t} \sum_{n, \omega_{1}}\left\langle m\left|\hat{\mu}_{\alpha} E_{\alpha}^{\omega_{1}} e^{-i \omega_{1} t^{\prime}} e^{\epsilon t^{\prime}}\right| n\right\rangle e^{i \omega_{m n} t^{\prime}} \\
\times \frac{1}{\hbar} \sum_{\omega_{2}} \frac{\left\langle n\left|\hat{\mu}_{\beta}\right| 0\right\rangle E_{\beta}^{\omega_{2}}}{\omega_{n 0}-\omega_{2}-i \epsilon} e^{i\left(\omega_{n 0}-\omega_{2}\right) t^{\prime}} e^{\epsilon t^{\prime}} d t^{\prime} \\
=\frac{1}{\hbar^{2}} \sum_{\omega_{1}, \omega_{2}} \sum_{n} \frac{\left\langle m\left|\hat{\mu}_{\alpha}\right| n\right\rangle\left\langle n\left|\hat{\mu}_{\beta}\right| 0\right\rangle E_{\alpha}^{\omega_{1}} E_{\beta}^{\omega_{2}}}{\left(\omega_{m 0}-\omega_{1}-\omega_{2}-i 2 \epsilon\right)\left(\omega_{n 0}-\omega_{2}-i \epsilon\right)} \\
d_{m}^{(3)}(t)=-\frac{1}{i \hbar} \int_{-\infty}^{t} \sum_{n, \omega_{1}}\left\langle e^{i\left(\omega_{m 0}-\omega_{1}-\omega_{2}\right) t} e^{2 \epsilon t}\right. \\
\times \frac{1}{\hbar^{2}} \sum_{\omega_{2}, \omega_{3}} \sum_{p} \frac{\left\langle n\left|\hat{\mu}_{\alpha} E_{\alpha}^{\omega_{1}} e^{-i \omega_{1} t^{\prime}} e^{\epsilon t^{\prime}}\right| n\right\rangle e^{i \omega_{m n} t^{\prime}}}{\left(\omega_{n 0}-\omega_{2}-\omega_{3}-i 2 \epsilon\right)\left\langle p\left|\hat{\mu}_{\gamma}\right| 0\right\rangle E_{\beta}^{\omega_{2}} E_{\gamma}^{\omega_{3}}} \\
\sum_{\left.\omega_{p 0}-\omega_{3}-i \epsilon\right)} e^{i\left(\omega_{n 0}-\omega_{2}-\omega_{3}\right) t^{\prime}} e^{2 \epsilon t^{\prime}} d t^{\prime} \\
\sum_{\omega_{1}, \omega_{2}, \omega_{3}} \frac{\left\langle m\left|\hat{\mu}_{\alpha}\right| n\right\rangle\left\langle n\left|\hat{\mu}_{\beta}\right| p\right\rangle\left\langle p\left|\hat{\mu}_{\gamma}\right| 0\right\rangle E_{\alpha}^{\omega_{1}} E_{\beta}^{\omega_{2}} E_{\gamma}^{\omega_{3}}}{\left(\omega_{m 0}-\omega_{1}-\omega_{2}-\omega_{3}-i 3 \epsilon\right)\left(\omega_{n 0}-\omega_{2}-\omega_{3}-i 2 \epsilon\right)\left(\omega_{p 0}-\omega_{3}-i \epsilon\right)} \\
\times e^{i\left(\omega_{m 0}-\omega_{1}-\omega_{2}-\omega_{3}\right) t} e^{3 \epsilon t} \quad
\end{gathered}
$$

substituindo esses coeficientes nas equações (17) podemos encontrar a polarizabilidade e as hiperpolarizabilidades que serão expressas a seguir pela equação (18).

A equação (10) reescrita utilizando as expressões (11) e (13) leva a

$$
\begin{aligned}
& |\Psi(t)\rangle=\left|\Psi^{(0)}(t)\right\rangle+\left|\Psi^{(1)}(t)\right\rangle+\left|\Psi^{(2)}(t)\right\rangle+\left|\Psi^{(3)}(t)\right\rangle+\cdots \\
& \left|\Psi^{(N)}(t)\right\rangle=\sum_{n} d_{n}^{(N)}(t) e^{-i E_{n} t / \hbar}|n\rangle .
\end{aligned}
$$

A polarização molecular, e consecutivamente a polarizabilidade e as hiperpolarizabilidades, estão relacionadas com o valor esperado do momento de dipolo. Portanto, podemos obter os termos de n-ésima ordem do valor esperado do momento de dipolo como

\footnotetext{
${ }^{1}$ As letras gregas serão utilizadas como sub índice para representar os eixos moleculares $\left(E_{\alpha}\right)$, os tensores serão grifados em negrito $(\boldsymbol{\beta})$ e os elementos dos tensores, definidos na próxima seção, serão representados com sub índices e parâmetros de frequências $\left(\gamma_{\alpha \beta \gamma \delta}\left(-\omega_{\sigma} ; \omega_{1}, \omega_{2}, \omega_{3}\right)\right)$.
} 


$$
\begin{aligned}
\langle\Psi(t)|\hat{\mu}| \Psi(t)\rangle & =\left\langle\Psi^{(0)}|\hat{\mu}| \Psi^{(0)}\right\rangle \\
& +\left\langle\Psi^{(0)}|\hat{\mu}| \Psi^{(1)}\right\rangle+\left\langle\Psi^{(1)}|\hat{\mu}| \Psi^{(0)}\right\rangle \\
& +\left\langle\Psi^{(0)}|\hat{\mu}| \Psi^{(2)}\right\rangle+\left\langle\Psi^{(1)}|\hat{\mu}| \Psi^{(1)}\right\rangle+\left\langle\Psi^{(2)}|\hat{\mu}| \Psi^{(0)}\right\rangle \\
& +\left\langle\Psi^{(0)}|\hat{\mu}| \Psi^{(3)}\right\rangle+\left\langle\Psi^{(1)}|\hat{\mu}| \Psi^{(2)}\right\rangle+\left\langle\Psi^{(2)}|\hat{\mu}| \Psi^{(1)}\right\rangle \\
& +\left\langle\Psi^{(3)}|\hat{\mu}| \Psi^{(0)}\right\rangle \\
& +\ldots \\
& =\langle\hat{\mu}\rangle^{(0)}+\langle\hat{\mu}\rangle^{(1)}+\langle\hat{\mu}\rangle^{(2)}+\langle\hat{\mu}\rangle^{(3)}+\cdots
\end{aligned}
$$

\subsubsection{Polarizabilidade linear}

A polarizabilidade linear está relacionada com a correção de primeira ordem do valor esperado do momento de dipolo. Portanto, omitindo algumas passagens matemáticas, $\langle\hat{\mu}\rangle^{(1)}$ é dado por

$$
\begin{aligned}
& \langle\hat{\mu}\rangle^{(1)}=\left\langle\Psi^{(0)}|\hat{\mu}| \Psi^{(1)}\right\rangle+\left\langle\Psi^{(1)}|\hat{\mu}| \Psi^{(0)}\right\rangle \\
& =\frac{1}{\hbar} \sum_{\omega_{1}} \sum_{n}\left[\frac{\left\langle 0\left|\hat{\mu}_{\alpha}\right| n\right\rangle\left\langle n\left|\hat{\mu}_{\beta}\right| 0\right\rangle}{\omega_{n 0}-\omega_{1}-i \epsilon}+\frac{\left\langle 0\left|\hat{\mu}_{\beta}\right| n\right\rangle\left\langle n\left|\hat{\mu}_{\alpha}\right| 0\right\rangle}{\omega_{n 0}+\omega_{1}+i \epsilon}\right] E_{\beta}^{\omega_{1}} e^{-i \omega_{1} t} e^{\epsilon t}
\end{aligned}
$$

Comparando as equações (8) e (19) relacionamos as componentes do tensor de polarizabilidade $\boldsymbol{\alpha}$ com a formulação da mecânica quântica. O parâmetro infinitesimal $\epsilon$ utilizado para evitar singularidades durante a integração e divergências da função resposta pode ser removido nesta etapa fazendo $\epsilon \rightarrow 0$. Portanto os elementos do tensor $\boldsymbol{\alpha}$ são dados por

$$
\alpha_{\alpha \beta}(-\omega ; \omega)=\frac{1}{\hbar} \sum_{n}\left[\frac{\left\langle 0\left|\hat{\mu}_{\alpha}\right| n\right\rangle\left\langle n\left|\hat{\mu}_{\beta}\right| 0\right\rangle}{\omega_{n 0}-\omega}+\frac{\left\langle 0\left|\hat{\mu}_{\beta}\right| n\right\rangle\left\langle n\left|\hat{\mu}_{\alpha}\right| 0\right\rangle}{\omega_{n 0}+\omega}\right] .
$$

A equação (20) além de representar a polarizabilidade está relacionada com as energias de excitações eletrônicas e com os momentos de dipolo de transição. As singularidades que surgem quando $\omega= \pm \omega_{n 0}$, chamadas de ressonâncias, são utilizadas para obter as energias de excitações utilizando o resíduo da função resposta descrita pela equação (20). O momento de dipolo de transição entre o estado fundamental e o estado excitado $f$, relacionado com a probabilidade de excitação entre esses estados, pode ser obtido pelo resíduo da função

$$
\lim _{\omega \rightarrow \omega_{f o}}\left(\omega_{f 0}-\omega\right) \alpha_{\alpha \beta}(-\omega ; \omega)=\left\langle 0\left|\hat{\mu}_{\alpha}\right| f\right\rangle\left\langle f\left|\hat{\mu}_{\beta}\right| 0\right\rangle .
$$

Uma consequência negativa ao assumir a perturbação como um campo elétrico clássico é a ausência do tempo de vida dos autos estados $|n\rangle$. Uma forma fenomenológica de incluir esse 
efeito é incorporar a taxa de decaimento populacional, $\Gamma_{n 0}$, na energia de excitação fazendo $\omega_{n 0} \rightarrow \omega_{n 0}+i \Gamma_{n 0} / 2$. O espectro de absorção linear está relacionado com a parte imaginaria da equação (20) e desta definição de taxa de decaimento é fácil verificar que a parte imaginária representa uma função do tipo Lorentziana.

\subsubsection{Hiperpolarizabilidade de primeira ordem}

Substituindo agora a equação (15) em (17) e (18) podemos obter a expressão matemática que representa a correção de segunda ordem da polarização. Para garantir a simetria de $\boldsymbol{\beta}$ introduz-se o operador $1 / 2 \sum \mathbb{P}_{1,2}$, o qual realiza a soma dos termos obtidos pela permutação dos pares $\left(\beta, \omega_{1}\right)$ e $\left(\gamma, \omega_{2}\right)$ sem alterar o resultado numérico.

$$
\begin{aligned}
& \langle\hat{\mu}\rangle^{(2)}=\left\langle\Psi^{(0)}|\hat{\mu}| \Psi^{(2)}\right\rangle+\left\langle\Psi^{(1)}|\hat{\mu}| \Psi^{(1)}\right\rangle+\left\langle\Psi^{(2)}|\hat{\mu}| \Psi^{(0)}\right\rangle \\
& =\frac{1}{2 \hbar^{2}} \sum_{\omega_{1}, \omega_{2}} \sum_{\mathbb{P}_{1,2}} \sum_{n, p}\left[\frac{\left\langle 0\left|\hat{\mu}_{\alpha}\right| n\right\rangle\left\langle n\left|\hat{\mu}_{\beta}\right| p\right\rangle\left\langle p\left|\hat{\mu}_{\gamma}\right| 0\right\rangle}{\left(\omega_{n 0}-\omega_{1}-\omega_{2}-i 2 \epsilon\right)\left(\omega_{p 0}-\omega_{2}-i \epsilon\right)}\right. \\
& +\frac{\left\langle 0\left|\hat{\mu}_{\beta}\right| n\right\rangle\left\langle n\left|\hat{\mu}_{\alpha}\right| p\right\rangle\left\langle p\left|\hat{\mu}_{\gamma}\right| 0\right\rangle}{\left(\omega_{n 0}+\omega_{1}+i \epsilon\right)\left(\omega_{p 0}-\omega_{2}-i \epsilon\right)} \\
& \left.+\frac{\left\langle 0\left|\hat{\mu}_{\gamma}\right| p\right\rangle\left\langle p\left|\hat{\mu}_{\beta}\right| n\right\rangle\left\langle n\left|\hat{\mu}_{\alpha}\right| 0\right\rangle}{\left(\omega_{n 0}+\omega_{1}+\omega_{2}+i 2 \epsilon\right)\left(\omega_{p 0}+\omega_{2}+i \epsilon\right)}\right] E_{\beta}^{\omega_{1}} E_{\gamma}^{\omega_{2}} e^{-i\left(\omega_{1}+\omega_{2}\right) t} e^{2 \epsilon t} .
\end{aligned}
$$

Comparando as equações (22) e (8) e novamente assumindo condições não ressonantes podemos identificar

$$
\beta_{\alpha \beta \gamma}\left(-\omega_{\sigma} ; \omega_{1}, \omega_{2}\right)=\frac{1}{\hbar^{2}} \sum \mathbb{P}_{-\sigma, 1,2} \sum_{n, p} \frac{\left\langle 0\left|\hat{\mu}_{\alpha}\right| n\right\rangle\left\langle n\left|\hat{\mu}_{\beta}\right| p\right\rangle\left\langle p\left|\hat{\mu}_{\gamma}\right| 0\right\rangle}{\left(\omega_{n 0}-\omega_{\sigma}\right)\left(\omega_{p 0}-\omega_{2}\right)}
$$

onde $\omega_{\sigma}=\omega_{1}+\omega_{2}$ e $\sum \mathbb{P}_{-\sigma, 1,2}$ é a soma de seis termos gerados pela permutação entre os pares $\left(\alpha,-\omega_{\sigma}\right),\left(\beta, \omega_{1}\right)$ e $\left(\gamma, \omega_{2}\right)$.

A expressão (23) contém informações sobre as energias de excitação, momentos de dipolo de transição do estado fundamental para estados excitados, momentos de dipolo de transição entre estados excitados e sobre os momentos de dipolo permanente. Algumas dessas propriedades podem ser extraídas utilizando os resíduos simples e duplos das funções respostas. $\mathrm{O}$ resíduo simples na condição de $\omega_{1}=-\omega_{f 0} / 2$ representa os elementos da matriz de transição de dois fótons 


$$
\begin{aligned}
\lim _{\omega_{2} \rightarrow \omega_{f 0}}\left(\omega_{f 0}-\omega_{2}\right) \beta_{\alpha \beta \gamma}\left(-\omega_{\sigma} ; \omega_{1}, \omega_{2}\right) & \\
= & \frac{1}{\hbar^{2}} \sum_{n}\left[\frac{\left\langle 0\left|\hat{\mu}_{\alpha}\right| n\right\rangle\left\langle n\left|\hat{\mu}_{\beta}\right| f\right\rangle}{\left(\omega_{n f}-\omega_{1}\right)}+\frac{\left\langle 0\left|\hat{\mu}_{\beta}\right| n\right\rangle\left\langle n\left|\hat{\mu}_{\alpha}\right| f\right\rangle}{\left(\omega_{n 0}+\omega_{1}\right)}\right]\left\langle f\left|\hat{\mu}_{\gamma}\right| 0\right\rangle .
\end{aligned}
$$

Considerando agora o resíduo duplo com $\omega_{1}=-\omega_{f 0}$ e $\omega_{2}=\omega_{g 0}$, com $|g\rangle$ representando um estado excitado, então se conhecermos o momento de dipolo de transição entre o estado fundamental e o estado excitado $|f\rangle$, obtemos utilizando a equação (25): o momento de dipolo de transição entre estados excitados quando $|f\rangle \neq|g\rangle$; ou o momento de dipolo permanente do estado excitado quando $|f\rangle=|g\rangle$.

$$
\begin{array}{r}
\lim _{\omega_{1} \rightarrow-\omega_{f 0}}\left(\omega_{f 0}+\omega_{1}\right)\left[\lim _{\omega_{2} \rightarrow \omega_{g 0}}\left(\omega_{g 0}-\omega_{2}\right) \beta_{\alpha \beta \gamma}\left(-\omega_{\sigma} ; \omega_{1}, \omega_{2}\right)\right] \\
=\left\langle 0\left|\hat{\mu}_{\beta}\right| f\right\rangle\left\langle f\left|\left(\hat{\mu}_{\alpha}-\left\langle 0\left|\hat{\mu}_{\alpha}\right| 0\right\rangle \delta_{f g}\right)\right| g\right\rangle\left\langle g\left|\hat{\mu}_{\gamma}\right| 0\right\rangle
\end{array}
$$

\subsubsection{Hiperpolarizabilidade de segunda ordem}

Substituindo a equação (16) nas equações (17) e (18) obtemos a expressão matemática que representa a correção de terceira ordem da polarização. Para garantir a simetria de $\gamma$ introduz-se o operador $1 / 6 \sum \mathbb{P}_{1,2,3}$ o qual realiza a soma dos termos obtidos pela permutação dos pares $\left(\beta, \omega_{1}\right),\left(\gamma, \omega_{2}\right)$ e $\left(\delta, \omega_{3}\right)$ sem alterar o resultado numérico.

$$
\begin{aligned}
& \langle\hat{\mu}\rangle^{(3)}=\left\langle\Psi^{(0)}|\hat{\mu}| \Psi^{(3)}\right\rangle+\left\langle\Psi^{(1)}|\hat{\mu}| \Psi^{(2)}\right\rangle+\left\langle\Psi^{(2)}|\hat{\mu}| \Psi^{(1)}\right\rangle+\left\langle\Psi^{(3)}|\hat{\mu}| \Psi^{(0)}\right\rangle \\
& =\frac{1}{6 \hbar^{3}} \sum_{\omega_{1}, \omega_{2}, \omega_{3}} \sum \mathbb{P}_{1,2,3} \sum_{n, m, p} \\
& {\left[\frac{\left\langle 0\left|\hat{\mu}_{\alpha}\right| n\right\rangle\left\langle n\left|\hat{\mu}_{\beta}\right| m\right\rangle\left\langle m\left|\hat{\mu}_{\gamma}\right| p\right\rangle\left\langle p\left|\hat{\mu}_{\delta}\right| 0\right\rangle}{\left(\omega_{n 0}-\omega_{1}-\omega_{2}-\omega_{3}-i 3 \epsilon\right)\left(\omega_{m 0}-\omega_{2}-\omega_{3}-i 2 \epsilon\right)\left(\omega_{p 0}-\omega_{3}-i \epsilon\right)}\right.} \\
& +\frac{\left\langle 0\left|\hat{\mu}_{\beta}\right| n\right\rangle\left\langle n\left|\hat{\mu}_{\alpha}\right| m\right\rangle\left\langle m\left|\hat{\mu}_{\gamma}\right| p\right\rangle\left\langle p\left|\hat{\mu}_{\delta}\right| 0\right\rangle}{\left(\omega_{n 0}+\omega_{1}+i \epsilon\right)\left(\omega_{m 0}-\omega_{2}-\omega_{3}-i 2 \epsilon\right)\left(\omega_{p 0}-\omega_{3}-i \epsilon\right)} \\
& +\frac{\left\langle 0\left|\hat{\mu}_{\gamma}\right| m\right\rangle\left\langle m\left|\hat{\mu}_{\beta}\right| n\right\rangle\left\langle n\left|\hat{\mu}_{\alpha}\right| p\right\rangle\left\langle p\left|\hat{\mu}_{\delta}\right| 0\right\rangle}{\left(\omega_{n 0}+\omega_{1}+\omega_{2}+i 2 \epsilon\right)\left(\omega_{m 0}+\omega_{2}+i \epsilon\right)\left(\omega_{p 0}-\omega_{3}-i \epsilon\right)} \\
& \left.+\frac{\left\langle 0\left|\hat{\mu}_{\delta}\right| p\right\rangle\left\langle p\left|\hat{\mu}_{\gamma}\right| m\right\rangle\left\langle m\left|\hat{\mu}_{\beta}\right| n\right\rangle\left\langle n\left|\hat{\mu}_{\alpha}\right| 0\right\rangle}{\left(\omega_{n 0}+\omega_{1}+\omega_{2}+\omega_{3}+i 3 \epsilon\right)\left(\omega_{m 0}+\omega_{2}+\omega_{3}+i 2 \epsilon\right)\left(\omega_{p 0}+\omega_{3}+i \epsilon\right)}\right] \\
& \times E_{\beta}^{\omega_{1}} E_{\gamma}^{\omega_{2}} E_{\delta}^{\omega_{3}} e^{-i\left(\omega_{1}+\omega_{2}+\omega_{3}\right) t} e^{3 \epsilon t} .
\end{aligned}
$$

Comparando a equação (26) com a equação (8) e novamente assumindo condições não ressonantes podemos identificar 


$$
\begin{aligned}
& \gamma_{\alpha \beta \gamma \delta}\left(-\omega_{\sigma} ; \omega_{1}, \omega_{2}, \omega_{3}\right) \\
& =\frac{1}{\hbar^{3}} \sum \mathbb{P}_{-\sigma, 1,2,3} \sum_{n, m, p} \frac{\left\langle 0\left|\hat{\mu}_{\alpha}\right| n\right\rangle\left\langle n\left|\hat{\mu}_{\beta}\right| m\right\rangle\left\langle m\left|\hat{\mu}_{\gamma}\right| p\right\rangle\left\langle p\left|\hat{\mu}_{\delta}\right| 0\right\rangle}{\left(\omega_{n 0}-\omega_{\sigma}\right)\left(\omega_{m 0}-\omega_{2}-\omega_{3}\right)\left(\omega_{p 0}-\omega_{3}\right)}
\end{aligned}
$$

onde $\omega_{\sigma}=\omega_{1}+\omega_{2}+\omega_{3}$ e $\sum \mathbb{P}_{-\sigma, 1,2,3}$ é a soma de 24 termos gerados pela permutação entre os pares $\left(\alpha,-\omega_{\sigma}\right),\left(\beta, \omega_{1}\right),\left(\gamma, \omega_{2}\right)$ e $\left(\delta, \omega_{3}\right)$.

A existência de três produtos no denominador da equação (27) resulta na existência de resíduos simples, duplos e triplos. Algumas propriedades eletrônicas podem ser obtidas utilizando as condições ressonantes com os resíduos da função resposta. Considerando o resíduo simples: assumindo $\omega_{1}=\omega_{2}=-\omega_{f 0} / 3$ e fazendo $\omega_{3} \rightarrow \omega_{f 0}$ é possível obter os elementos de matriz de transição de três fótons de mesma energia entre o estado fundamental e excitado (equação (28)). Considerando o resíduo duplo: obtemos os elementos de matriz de transição de dois fótons de mesma energia entre estados excitados fazendo $\omega_{1}=-\omega_{f g} / 2$ nos limites $\omega_{3} \rightarrow \omega_{f 0}$ e $\omega_{2} \rightarrow \omega_{g 0}$ (equação (29)). Considerando novamente o resíduo duplo: obtemos a polarizabilidade $\boldsymbol{\alpha}$ para o estado excitado assumindo os limites $\omega_{3} \rightarrow \omega_{f 0}$ e $\omega_{2} \rightarrow$ $-\omega_{f 0}($ equação (30)).

$$
\begin{aligned}
\lim _{\omega_{3} \rightarrow \omega_{f 0}}\left(\omega_{f 0}-\omega_{3}\right) & \gamma_{\alpha \beta \gamma \delta}\left(-\omega_{\sigma} ; \omega_{1}, \omega_{2}, \omega_{3}\right) \\
= & \frac{1}{\hbar^{3}} \sum \mathbb{P}_{-\sigma, 1,2} \sum_{n, m} \frac{\left\langle 0\left|\hat{\mu}_{\alpha}\right| n\right\rangle\left\langle n\left|\hat{\mu}_{\beta}\right| m\right\rangle\left\langle m\left|\hat{\mu}_{\gamma}\right| f\right\rangle}{\left(\omega_{n f}-\omega_{1}-\omega_{2}\right)\left(\omega_{m f}-\omega_{2}\right)}\left\langle f\left|\hat{\mu}_{\delta}\right| 0\right\rangle \\
\lim _{\omega_{2} \rightarrow \omega_{g 0}}\left(\omega_{g 0}-\right. & \left.\omega_{2}\right)\left[\lim _{\omega_{3} \rightarrow \omega_{f 0}}\left(\omega_{f 0}-\omega_{3}\right) \gamma_{\alpha \beta \gamma \delta}\left(-\omega_{\sigma} ; \omega_{1}, \omega_{2}, \omega_{3}\right)\right] \\
& =\frac{1}{\hbar^{3}} \sum_{n}\left[\frac{\left\langle g\left|\hat{\mu}_{\alpha}\right| n\right\rangle\left\langle n\left|\hat{\mu}_{\beta}\right| f\right\rangle}{\left(\omega_{n f}-\omega_{1}\right)}\right. \\
& +\frac{\left.\left\langle g\left|\hat{\mu}_{\beta}\right| n\right\rangle\left\langle n\left|\hat{\mu}_{\alpha}\right| f\right\rangle\right]\left\langle 0\left|\hat{\mu}_{\gamma}\right| g\right\rangle\left\langle f\left|\hat{\mu}_{\delta}\right| 0\right\rangle .}{\left(\omega_{n g}+\omega_{1}\right)} \\
\lim _{\omega_{2} \rightarrow-\omega_{f 0}}\left(\omega_{f 0}+\right. & \left.\omega_{2}\right)\left[\lim _{\omega_{3} \rightarrow \omega_{f 0}}\left(\omega_{f 0}-\omega_{3}\right) \gamma_{\alpha \beta \gamma \delta}\left(-\omega_{\sigma} ; \omega_{1}, \omega_{2}, \omega_{3}\right)\right] \\
& =\frac{1}{\hbar^{3}} \sum_{\mathbb{P}_{-\sigma, 1}\left[\sum_{m \neq f} \frac{\left\langle f\left|\hat{\mu}_{\alpha}\right| m\right\rangle\left\langle m\left|\hat{\mu}_{\beta}\right| f\right\rangle}{\left(\omega_{m f}-\omega_{1}\right)}\right.} \\
& \left.-\sum_{n \neq 0} \frac{\left.\left\langle 0\left|\hat{\mu}_{\alpha}\right| n\right\rangle\left\langle n\left|\hat{\mu}_{\beta}\right| 0\right\rangle\right]\left\langle 0\left|\hat{\mu}_{\gamma}\right| f\right\rangle\left\langle f\left|\hat{\mu}_{\delta}\right| 0\right\rangle}{\left(\omega_{n 0}-\omega_{1}\right)}\right]
\end{aligned}
$$


As expressões acima são válidas considerando energias distantes das regiões de ressonância e é de grande importância destacar o caso ressonante. Nessa condição, novamente, introduz-se a taxa de decaimento populacional de forma fenomenológica fazendo $\omega_{f 0} \rightarrow \omega_{f 0}+$ $i \Gamma_{f 0} / 2$. No caso ressonante, existe somente a contribuição relacionada com a parte imaginária de $\gamma_{\alpha \beta \gamma \delta}(-\omega ; \omega,-\omega, \omega)$. Quando $\omega_{1}=\omega_{3}=-\omega_{2}$ e assumindo $\omega_{1}=\omega_{f 0} / 2=\omega$ podemos relacionar a parte imaginária da segunda hiperpolarizabilidade como o produto de elementos da matriz de transição de dois fótons.

$$
\begin{aligned}
\operatorname{Im}\left\{\gamma_{\alpha \beta \gamma \delta}(-\omega ; \omega,-\omega, \omega)\right\} & =\frac{1}{\hbar} \frac{2}{\Gamma_{f 0}}\left(\frac{1}{\hbar} \sum_{n}\left[\frac{\left\langle 0\left|\hat{\mu}_{\alpha}\right| n\right\rangle\left\langle n\left|\hat{\mu}_{\beta}\right| f\right\rangle}{\left(\omega_{n 0}-\omega\right)}+\frac{\left\langle 0\left|\hat{\mu}_{\beta}\right| n\right\rangle\left\langle n\left|\hat{\mu}_{\alpha}\right| f\right\rangle}{\left(\omega_{n 0}-\omega\right)}\right]\right) \\
& \times\left(\frac{1}{\hbar} \sum_{n}\left[\frac{\left\langle f\left|\hat{\mu}_{\gamma}\right| n\right\rangle\left\langle n\left|\hat{\mu}_{\delta}\right| 0\right\rangle}{\left(\omega_{n 0}-\omega\right)}+\frac{\left\langle f\left|\hat{\mu}_{\delta}\right| n\right\rangle\left\langle n\left|\hat{\mu}_{\gamma}\right| 0\right\rangle}{\left(\omega_{n 0}-\omega\right)}\right]\right)
\end{aligned}
$$

A análise das funções resposta é uma forma de obter propriedades de estados excitados utilizando a função de onda do estado fundamental. Com esta abordagem podemos obter, utilizando resíduos das funções respostas, o valor total da soma-sobre-estados evitando erros de truncamento. Contudo, erros devido a limitações da completude das funções bases e das representações dos orbitais moleculares pelos métodos de estrutura eletrônica ainda estão presentes.

\subsubsection{EFISHG e HRS}

A técnica experimental conhecida como Electric Field-Induced Second Harmonic Generation (EFISHG) foi desenvolvida para obter a primeira hiperpolarizabilidade de compostos em solução. Esta técnica consiste em aplicar um campo elétrico estático para quebrar a simetria de inversão espacial do meio. Essa redução de simetria ocorre devido ao alinhamento do momento de dipolo molecular com o campo elétrico. Resumidamente podemos afirmar que o resultado obtido com a técnica EFISHG representa o valor da hiperpolarizabilidade orientada na mesma direção do momento de dipolo permanente do estado fundamental da molécula. Embora não seja possível utilizar essa técnica em sistemas carregados ela tem fornecido resultados interessantes para complexos de pares iônicos. As expressões matemáticas abaixo descrevem as informações experimentais fornecidas pela técnica EFISHG [60]: 


$$
\begin{aligned}
\gamma_{E F I S H G} & =\langle\gamma(-2 \omega ; \omega, \omega, 0)\rangle \\
& =\gamma_{/ /}(-2 \omega ; \omega, \omega, 0)+\frac{\mu \beta_{/ /}(-2 \omega ; \omega, \omega)}{3 k T} \\
& =\frac{\left[\mu \beta_{/ /}(-2 \omega ; \omega, \omega)\right]_{e f f}}{3 k T}
\end{aligned}
$$

onde $\mu$ é o módulo do momento de dipolo permanente do estado fundamental, $\gamma_{E F I S H G}$ é a resposta EFISHG completa, $\mathrm{k}$ é a constante de Boltzmann e T é a temperatura absoluta. O termo $\gamma_{/ /}(-2 \omega ; \omega, \omega, 0)$ é a contribuição de terceira ordem projetada sobre o momento de dipolo $\mu$. Esse termo é definido pela equação

$$
\begin{aligned}
\gamma_{/ /}(-2 \omega ; \omega ; \omega ; 0) & =\gamma_{/ /} \\
& =\frac{1}{15} \sum_{\zeta, \eta}\left(\gamma_{\zeta \zeta \eta \eta}+\gamma_{\zeta \eta \eta \zeta}+\gamma_{\zeta \eta \zeta \eta}\right) \\
& =\frac{1}{15} \sum_{\zeta, \eta}\left(2 \gamma_{\zeta \zeta \eta \eta}+\gamma_{\zeta \eta \eta \zeta}\right)
\end{aligned}
$$

O termo $\beta_{/ /}(-2 \omega ; \omega, \omega) / 3 k T$ é a contribuição de segunda ordem da resposta EFISHG projetada sobre o momento de dipolo $\mu$, chamada de $\beta$ paralelo. Tal termo tem sido representado como $\beta_{\text {EFISHG }}(-2 \omega ; \omega, \omega)=\beta_{/ /}(-2 \omega ; \omega, \omega)=\beta / /$ :

$$
\beta_{/ /}(-2 \omega ; \omega, \omega)=\frac{1}{5} \frac{1}{\mu} \sum_{\alpha} \mu_{\alpha} \sum_{\beta}\left(\beta_{\alpha \beta \beta}+\beta_{\beta \alpha \beta}+\beta_{\beta \beta \alpha}\right)=\frac{3}{5} \frac{1}{\mu} \sum_{\alpha} \mu_{\alpha} \beta_{\alpha}
$$

onde $\mu_{\alpha}$ é a $\alpha$-ésima componente cartesiana do momento de dipolo e $\beta_{\alpha}$ é a $\alpha$-ésima componente cartesiana do vetor de hiperpolarizabilidade. A resposta efetiva $\left[\mu \beta_{/ /}\right]_{\text {eff }}$ pode ser obtida reagrupando os termos de segunda e terceira ordem, como definido na equação (32) permitindo comparação direta entre resultados teóricos e experimentais. Essa resposta efetiva é necessária quando as medidas experimentais são realizadas numa única temperatura, impossibilitando, do ponto de vista experimental, desacoplar os termos de segunda e terceira ordem.

Outra técnica para obter informações sobre a primeira hiperpolarizabilidade é conhecida como Hyper-Rayleigh Scattering (HRS), onde a luz espalhada é analisada num ângulo de $90^{\circ}$ em relação a direção de propagação do feixe incidente. Dessa análise é possível obter a taxa de despolarização (DR) a qual podemos inferir sobre o caráter multipolar (dipolar, quadrupolar etc.) da molécula. Essas propriedades são representadas por [61] 


$$
\begin{gathered}
\beta_{H R S}(-2 \omega ; \omega, \omega)=\sqrt{\left\langle\beta_{Z Z Z}^{2}\right\rangle+\left\langle\beta_{Z X X}^{2}\right\rangle} \\
\left\langle\beta_{Z Z Z}^{2}\right\rangle=\frac{1}{105} \sum_{\zeta \eta \chi}\left(2 \beta_{\zeta \eta \chi}^{2}+\beta_{\zeta \eta \eta} \beta_{\zeta \chi \chi}+4 \beta_{\zeta \zeta \eta} \beta_{\eta \chi \chi}+4 \beta_{\zeta \zeta \eta} \beta_{\chi \chi \eta}+4 \beta_{\zeta \eta \chi} \beta_{\eta \zeta \chi}\right) \\
\left\langle\beta_{Z X X}^{2}\right\rangle=\frac{1}{105} \sum_{\zeta \eta \chi}\left(6 \beta_{\zeta \eta \chi}^{2}+3 \beta_{\zeta \eta \eta} \beta_{\zeta \chi \chi}-2 \beta_{\zeta \zeta \eta} \beta_{\eta \chi \chi}-2 \beta_{\zeta \zeta \eta} \beta_{\chi \chi \eta}-2 \beta_{\zeta \eta \chi} \beta_{\eta \zeta \chi}\right) \\
D R=\frac{\left\langle\beta_{Z Z Z}^{2}\right\rangle}{\left\langle\beta_{Z X X}^{2}\right\rangle}
\end{gathered}
$$

Note que subíndices minúsculos se referem ao sistema de coordenadas molecular e os subíndices maiúsculos ao sistema de coordenadas do laboratório.

\subsubsection{Absorção de dois fótons}

Podemos encontrar a probabilidade $P$ da transição entre o estado fundamental $|0\rangle$ e o estado excitado $|f\rangle$ observando, da equação (10), que $P_{0 \rightarrow f}=\left|d_{f}(t)\right|^{2}$. A transição pode ocorrer pelo processo de $\mathrm{A} 1 \mathrm{~F}$, relacionado $\operatorname{com} d_{f}^{(1)}(t)$, ou pelo processo de $\mathrm{A} 2 \mathrm{~F}$, relacionado com $d_{f}^{(2)}(t)$. Quando o tempo $t$ é muito maior que $2 \pi / \omega_{f 0}$ é possível afirmar que o termo entre colchetes na equação (39) resulta numa distribuição estreita centrada em $\omega_{f 0}=\omega_{1}+\omega_{2}$. Isso significa que a A2F ocorre somente quando a soma da energia dos fótons incidentes é igual a energia da transição. Combinando somas em índices mudos para obter uma equação simétrica definimos o elemento da matriz de transição $S_{\alpha \beta}$ (entre o estado fundamental e o estado final $f$ ) como apresentado na equação (40). É interessante notar a similaridade entre as equações (40) e (24). Pela probabilidade de transição de dois fótons se relacionar com o quadrado de $d_{f}^{(2)}(t)$ ela apresenta uma dependência de quarta potência com o campo perturbativo, levando a uma dependência quadrática com a intensidade da radiação incidente.

$$
\begin{gathered}
d_{f}^{(2)}(t)=\frac{1}{\hbar^{2}} \sum_{\omega_{1}, \omega_{2}} \sum_{n} \frac{\left\langle f\left|\hat{\mu}_{\alpha}\right| n\right\rangle\left\langle n\left|\hat{\mu}_{\beta}\right| 0\right\rangle}{\omega_{n 0}-\omega_{2}} E_{\alpha}^{\omega_{1}} E_{\beta}^{\omega_{2}}\left[\frac{e^{i\left(\omega_{f 0}-\omega_{1}-\omega_{2}\right) t}}{\omega_{f 0}-\omega_{1}-\omega_{2}}\right] . \\
S_{\alpha \beta}=\frac{1}{\hbar} \sum_{n}\left[\frac{\left\langle 0\left|\hat{\mu}_{\alpha}\right| n\right\rangle\left\langle n\left|\hat{\mu}_{\beta}\right| f\right\rangle}{\omega_{n 0}-\omega_{1}}+\frac{\left\langle 0\left|\hat{\mu}_{\beta}\right| n\right\rangle\left\langle n\left|\hat{\mu}_{\alpha}\right| f\right\rangle}{\omega_{n 0}-\omega_{2}}\right] .
\end{gathered}
$$

Do ponto de vista macroscópico a seção de choque de A2F $\sigma$ depende do número de fótons absorvidos por unidade de tempo $d n_{p} / d t$ e do quadrado do fluxo de fótons $F^{2}$, onde $F=E E c / 2 \pi \omega$. Pode-se obter a taxa de fótons absorvidos computando a razão entre a taxa de variação da energia (equação (6)) e a frequência do feixe incidente 


$$
\sigma=\frac{d n_{p}}{d t} \frac{1}{F^{2}}=\frac{24 \pi^{2} \omega^{2}}{c^{2}} \operatorname{Im}\left\{\chi^{(3)}\right\}
$$

Para relacionar a origem microscópica à detecção macroscópica dos processos de $\mathrm{A} 2 \mathrm{~F}$ é necessário ajustar os dois sistemas de coordenadas, o do laboratório e o do sistema molecular. Sabendo que experimentos raramente são feitos em moléculas individuais, mas em um conjunto de moléculas que para gases ou líquidos não tem orientação preferencial, a relação entre os sistemas de coordenadas deve incluir uma orientação média completa. Adotando a média orientacional apresentada na referência [55], a probabilidade de transição via dois fótons passa a ser

$$
\delta=\langle\delta\rangle=\left\langle\left|S_{\alpha \beta}\right|^{2}\right\rangle=\frac{1}{30} \sum_{\alpha \beta}\left(c_{1} S_{\alpha \alpha} S_{\beta \beta}^{*}+c_{2} S_{\alpha \beta} S_{\alpha \beta}^{*}+c_{2} S_{\alpha \beta} S_{\beta \alpha}^{*}\right)
$$

onde os coeficientes $c_{i}$ estão relacionados com a polarização dos fótons incidentes, sendo todos iguais a 2 para fótons linearmente polarizados de mesma energia $\omega$. Neste caso a notação \langle\rangle representa a média orientacional.

Portanto podemos reescrever $\sigma$ em função da propriedade microscópica $\delta$ como

$$
\sigma=\frac{4 \pi^{3} \omega^{2}}{c^{2}} \delta g\left(2 \omega, \omega_{f 0}, \Gamma_{f 0}\right)
$$

onde $\omega=\omega_{f 0} / 2$ representa a energia dos fótons absorvidos e $c$ é a velocidade da luz no vácuo. A inclusão da função $g\left(2 \omega, \omega_{0}, \Gamma_{f 0}\right)$, moduladora de efeitos de alargamento espectral, é novamente uma forma fenomenológica de introduzir o decaimento populacional e efeitos vibracionais. Como vimos nas seções anteriores a inclusão de decaimento populacional de forma fenomenológica transformando $\omega_{f 0} \rightarrow \omega_{f 0}+i \Gamma_{f 0} / 2$ resulta numa função de alargamento Lorentziana. Portanto, é natural assumir que a função $g\left(2 \omega, \omega_{0}, \Gamma_{f 0}\right)$ seja também uma Lorentziana de largura de linha completa a meia altura $\Gamma_{f 0}$, definida como

$$
g\left(2 \omega, \omega_{0}, \Gamma_{f 0}\right)=\frac{1}{2 \pi} \frac{\Gamma_{f 0}}{\left(2 \omega-\omega_{f 0}\right)^{2}+0.25 \Gamma_{f 0}^{2}} .
$$

Finalmente a seção de choque de A2F na condição de ressonância, $2 \omega=\omega_{f 0}$, pode ser expressa por

$$
\sigma=\frac{8 \pi^{2} \omega^{2}}{15 c^{2} \Gamma_{f 0}} \sum_{\alpha \beta}\left(S_{\alpha \alpha} S_{\beta \beta}^{*}+2 S_{\alpha \beta} S_{\beta \alpha}^{*}\right)
$$


A unidade da seção de choque de A2F é GM em homenagem a Göppert-Mayer. 1 u.a. é igual $1.896788 \mathrm{GM}$ e $1 \mathrm{GM}$ representa $10^{-50} \mathrm{~cm}^{4} \mathrm{~s} /$ photon.

A expressão (40) envolve a soma-sobre-estados e em casos onde poucos estados contribuem majoritariamente no processo de A2F podemos simplificar essa soma utilizando modelos de poucos níveis. A aproximação mais simples considera somente dois níveis de energia: o estado fundamental e um estado excitado final, enquanto aproximações subsequentes consideram três níveis (estado fundamental e dois estados excitados) e assim por diante. A seção de choque de A2F para o modelo de dois níveis é descrita pela equação (46) e envolve o momento de dipolo de transição entre os estados fundamental e excitado $\mu_{0 f}$, a diferença entre os momentos de dipolo permanente destes estados $\Delta \mu_{0 f}$ e o ângulo formado pelos vetores $\mu_{0 f}$ e $\Delta \mu_{0 f}$. Os modelos de poucos níveis, além de possibilitar a obtenção da seção de choque de A2F utilizando métodos mais robustos, permite analisar as contribuições de cada estado de forma individual.

$$
\sigma^{2 L M}=\frac{16 \pi^{3}}{15 c^{2}}\left|\mu_{0 f}\right|^{2}\left|\Delta \mu_{0 f}\right|^{2}\left(2 \cos ^{2}\left(\theta_{\mu, \Delta \mu}\right)+1\right) g\left(2 \omega, \omega_{0}, \Gamma_{f 0}\right)
$$

$\mathrm{O}$ desenvolvimento das equações apresentadas para a seção de choque de A2F considerou fótons incidentes com a mesma energia provenientes de um único feixe laser. Essa definição teórica é consistente com a análise de dados e o aparato experimental da técnica conhecida como Z-scan de único feixe. Em tal técnica o feixe de luz da excitação é transmitido através de uma lente convergente e a posição da amostra investigada é variada ao longo da direção de propagação do feixe de luz (direção Z). Ao ser varrida naquela direção, e em torno da região focal do feixe, a amostra é submetida a um feixe de excitação focalizado cuja intensidade varia ao longo da direção Z. A transmitância não linear da luz incidente através da amostra é então registrada em função da posição da amostra. A partir da variação da transmitância pode-se então estimar o valor do coeficiente de absorção não linear do processo de A2F. Utilizando o valor determinado para esse coeficiente pode-se então, conhecendo a concentração da amostra, determinar a seção de choque de A2F da amostra para um comprimento de onda de excitação específico utilizando as equações (6) e (7).

\subsection{Métodos de física atômica e molecular}

Para estudar propriedades microscópicas e eletrônicas de átomos e moléculas é necessária a formulação da Mecânica Quântica. Bastaria resolver a equação de Schrödinger 
(47) para atingir tais objetivos, porém a matemática envolvida na solução desta equação é complexa e poucos são os problemas solúveis exatamente. Segue-se nessa seção uma breve introdução aos métodos de cálculos de estrutura eletrônica utilizados neste trabalho. Não temos pretensão de substituir os textos já renomados e recomendamos as seguintes leituras para estudos mais aprofundados [50,62-67].

$$
i \hbar \frac{\partial}{\partial t}|\Psi(\mathbf{r}, \mathrm{t})\rangle=\widehat{\mathrm{H}}(\mathbf{r}, \mathrm{t})|\Psi(\mathbf{r}, \mathrm{t})\rangle
$$

Para sistemas moleculares, ao assumir que os movimentos nuclear e eletrônico ocorrem em diferentes escalas de tempo e considerando a ausência de acoplamentos entre diferentes estados eletrônicos ou vibracionais é possível usar a aproximação de Born-Oppenheimer. Nessa aproximação, os movimentos nuclear e eletrônico são desacoplados devido à massa nuclear ser muito maior que a massa eletrônica. Consequentemente, as posições nucleares são incluídas parametricamente na energia eletrônica e na função de onda do estado eletrônico [68].

O método de Hartree-Fock (HF) foi um dos primeiros métodos propostos para resolver a parte eletrônica resultante do desacoplamento entre os núcleos e os elétrons. A função de onda eletrônica é aproximada por um único determinante de Slater [69] cujos elementos de matriz representam o spin e a região espacial dos elétrons, chamados de funções spin-orbitais. Neste método os elétrons são independentes e as interações elétron-elétron representam um campo médio, portanto, parte da energia eletrônica não é contabilizada. A diferença entre a energia obtida com o método HF e a energia exata é chamada de energia de correlação. Do desenvolvimento dessa metodologia surgem as integrais de um e de dois elétrons, cuja solução se dá utilizando um processo iterativo auto consistente conhecido como Self Consistent Field (SCF) [70].

Devido a limitações computacionais da época (em torno de 1950), principalmente relacionadas com o cálculo das integrais de dois elétrons, surgiram os métodos semi-empíricos. Diversas aproximações foram propostas baseadas na aproximação Zero Differential Overlap [71], entre elas o Complete Neglect Differential Overlap [72] e o Intermediate Neglect Differential Overlap com parametrização espectroscópica (INDO/S) [73] que se diferenciam pela quantidade de parâmetros e pela forma como estes parâmetros são obtidos. Embora o poder computacional tenha aumentado expressivamente comparado com o disponível em 1950, a simulação de sistemas mais realistas envolve sempre grande número de átomos e os métodos semi-empíricos ainda hoje são úteis para esses grandes sistemas. 
Tárcius N. Ramos

Por construção, o método HF não inclui energia de correlação eletrônica e para incorporar essa energia negligenciada surgiram os métodos conhecidos como pós-Hartree-Fock (pós-HF). Diversos métodos foram propostos como a teoria de perturbação, a interação de configurações e o Coupled Cluster. Porém esses métodos são custosos computacionalmente e alguns deles escalam com a sétima potência do número $\mathrm{N}$ de elétrons. Outra proposta para incluir a energia de correlação e, também, de reduzir o custo computacional surgiu com o desenvolvimento da Teoria do Funcional da Densidade (Density Functional Theory - DFT). $\mathrm{Na}$ DFT é possível representar a função de onda de $3 \mathrm{~N}$ variáveis, onde $\mathrm{N}$ é o número de elétrons do sistema, por uma densidade eletrônica representada por somente 3 variáveis espaciais.

\subsubsection{Teoria do funcional da densidade}

Os métodos pós-HF tornam-se inviáveis computacionalmente para estudar sistemas moleculares com elevada quantidade de elétrons. Portanto, o desenvolvimento de metodologias capazes de obter a estrutura eletrônica de sistemas moleculares às custas de menor demanda computacional se fez necessária. Na década de 1960, os trabalhos de Hohenberg e Kohn (HK) [74] e de Kohn e Sham (KS) [75] apresentaram o cálculo das propriedades eletrônicas de sistema composto por gás de elétrons utilizando a DFT. A ideia da DFT é buscar uma densidade eletrônica $\rho$ representada por 3 variáveis que reproduza a densidade de uma função de onda de $3 N$ variáveis. Em 1964, HK demonstraram os teoremas que validam a DFT, garantindo uma relação unívoca entre a densidade eletrônica, a função de estado e a energia do estado fundamental utilizando o princípio variacional. No entanto, até aquele momento ainda não havia sido apresentada uma forma de se utilizar a densidade eletrônica para encontrar propriedades moleculares. No ano seguinte, KS apresentaram uma maneira de obter a densidade eletrônica $\rho$ utilizando a abordagem SCF para a solução de funções orbitais $\psi_{i}(\boldsymbol{r})$ com energias $\epsilon_{i}$

$$
\begin{gathered}
(\underbrace{-\frac{1}{2} \nabla^{2}+v^{K S}[\rho]}_{h^{K S}}) \psi_{i}(\boldsymbol{r})=\epsilon_{i} \psi_{i}(\boldsymbol{r}), \\
v^{K S}[\rho]=v(\boldsymbol{r})+\int \frac{\rho\left(\boldsymbol{r}^{\prime}\right)}{\left|\boldsymbol{r}-\boldsymbol{r}^{\prime}\right|} d \boldsymbol{r}^{\prime}+\frac{\delta E_{x c}[\rho]}{\delta \rho}
\end{gathered}
$$

onde $v^{K S}$ é o potencial efetivo de KS formado: pelo potencial externo $v(\boldsymbol{r})$ sentido pelos elétrons devido à presença dos núcleos, pelo termo conhecido como potencial de Hartree, e, finalmente, pelo termo relacionado com o funcional de troca-correlação $\left(E_{x c}[\rho]\right)$.

A solução da equação de KS é a priori exata e o processo SCF é tal que: dada uma densidade $\rho$ inicial, construímos o potencial efetivo $v^{K S}$ e podemos encontrar o estado $\psi$ que 
fornecerá uma nova densidade. O ciclo se repete até atingir um critério de convergência préestabelecido. Além disso, o processo de convergência deve satisfazer o teorema variacional. $\mathrm{O}$ potencial efetivo $v^{K S}$ depende da energia de troca-correlação $\left(E_{x c}[\rho]\right)$, a qual não é conhecida. O termo $E_{x c}[\rho]$ é responsável pelas aproximações no método DFT e não existe um consenso em como o obter. Existem muitas propostas para $E_{x c}[\rho]$ conhecidas como funcionais de troca e correlação ou simplesmente funcionais DFT e a acurácia dos resultados estão ligados à boa descrição do termo de $E_{x c}[\rho]$, sendo o ponto crítico do desenvolvimento da teoria. Os funcionais de troca e correlação B3LYP, BLYP-D3, BHandHLYP, CAM-B3LYP e $\omega B$ B7X-D foram os funcionais utilizados neste trabalho:

- B3LYP [76]: Entre os funcionais mais utilizados atualmente, o B3LYP é um funcional híbrido que mistura a energia de diferentes funcionais DFT com uma contribuição da energia exata de troca obtida com HF. Dado por

$$
E_{x c}^{B 3 L Y P}=a E_{x}^{L S D A}+(1-a) E_{x}^{H F}+b \Delta E_{x}^{B 88}+c E_{c}^{L Y P}+(1-c) E_{c}^{V W N}
$$

onde $a=0.2, b=0.72$ e $c=0.81$ são coeficientes determinados por um ajuste experimental, $E_{x}^{L S D A}$ é o funcional de troca baseado em Local Spin Density Approximation, $E_{x}^{H F}$ é a energia de troca exata de HF, $\Delta E_{x}^{B 88}$ é a correção do gradiente de Becke, $E_{c}^{L Y P}$ é a energia de correlação de Lee, Yang e Par e $E_{c}^{V W N}$ é a energia de correlação Vosko-Wilk-Nusair.

- BLYP-D3 [77-79]: O funcional BLYP é a combinação dos funcionais de troca LSDA corrigido com o gradiente de Becke e de correlação de LYP. Adicionalmente o termo de correção de dispersão D3 [79] é incluído.

$$
E_{x C}^{B L Y P}=E_{x}^{L S D A}+\Delta E_{x}^{B 88}+E_{c}^{L Y P}-E_{D 3}
$$

- CAM-B3LYP [80]: Este funcional utiliza o Coulomb Attenuating Method (CAM) para separar os termos de curto (SR) e de longo (LR) alcance utilizando dois parâmetros $\alpha$ e $\beta$

$$
\frac{1}{r}=\underbrace{\frac{1-[\alpha+\beta \operatorname{erf}(\mu r)]}{r}}_{S R}+\underbrace{\frac{\alpha+\beta \operatorname{erf}(\mu r)}{r}}_{L R} .
$$

Esses dois parâmetros misturam as contribuições do termo de HF no B3LYP tanto para SR quanto para LR. Quando $\alpha=0.2$ e $\beta=0$ recupera-se o funcional B3LYP e assintoticamente $65 \%$ do termo de HF é incluído. Os parâmetros definidos são: $\alpha=0.19, \beta=0.46$ e $\mu=0.33$.

- BHandHLYP [81]: O funcional apresenta uma contribuição dos termos de HF, LSDA e B88 para o termo de troca e o funcional LYP para o termo de correlação 


$$
E_{x C}^{B H \& H L Y P}=0.5 E_{x c}^{L S D A}+0.5 E_{x}^{H F}+0.5 \Delta E_{x}^{B 88}+E_{c}^{L Y P} .
$$

- $\quad \omega B 97 X-D$ [82,83]: A proposta dos autores é ir além da inclusão dos termos de separação de SR/LR (com $\alpha=0$ e $\beta=1$ ), re-otimizando o funcional de Becke B97 [84] e adicionando um termo de dispersão

$$
\begin{gathered}
E_{x c}^{w B 97 X D}=E_{x}^{L R-H F}+0,222036 E_{x}^{S R-H F}+E_{x}^{S R-B 97}+E_{c}^{B 97}+E_{d i s p} \\
E_{d i s p}=-\sum_{i=1}^{N-1} \sum_{j=i+1}^{N} \frac{C_{6}^{i j}}{R_{i j}^{6}} \frac{1}{1+a\left(\frac{R_{i j}}{R_{r}}\right)^{-12}}
\end{gathered}
$$

onde $C_{6}^{i j}$ é o coeficiente de dispersão para o par de átomos $i j$ e $R_{i j}$ a distância entre o mesmo par. $R_{r}$ é a soma do raio de van der Waals do par atômico e $a$ é um parâmetro para controlar a dispersão.

\subsubsection{Teoria do funcional da densidade dependente do tempo}

Devido ao relativo sucesso da DFT para cálculos de estrutura eletrônica do estado fundamental e diante da necessidade de compreender as propriedades dos estados excitados, surgiu a ideia de estender a DFT para o formalismo dependente do tempo. Em 1984, Runge e Gross (RG) [85] demonstraram dependência unívoca entre a densidade dependente do tempo $\rho(\boldsymbol{r}, t)$ e o potencial externo dependente do tempo $v(\boldsymbol{r}, t)$ utilizando a equação de Schrödinger dependente do tempo, assim surgiu a Time Dependent Density Functional Theory (TD-DFT). Para detalhes da demonstração recomenda-se a leitura do artigo original.

Se encontrar a solução para o problema estacionário é um desafio, obter a solução para equações dependentes do tempo é uma tarefa ainda mais complexa. Contudo, em muitos casos não é necessário obter a solução explicitamente dependente do tempo e essa é uma das motivações para o desenvolvimento da TD-DFT. A utilização das funções respostas, como descrita considerando a solução exata e conhecida (ver seção 2.2), é uma possibilidade para obter energias de excitação e momentos de transição entre estados eletrônicos. A teoria das funções de resposta é um método elaborado da teoria da perturbação dependente do tempo. $\mathrm{O}$ formalismo é analiticamente transmissível, ou seja, pode ser aplicado para obter propriedades de estados excitados utilizando funções de onda monodeterminantais como HF e DFT. Uma das vantagens desse formalismo comparado com métodos derivativos é a redução de uma ordem de perturbação, ou seja, a resposta linear está associada com as grandezas de segunda ordem em energia. 
A seguir descrevemos de forma sucinta os procedimentos para obtenção da função resposta no formalismo da TD-DFT e como eles se relacionam com os processos de absorção de um e de dois fótons.

O estado de referência dependente do tempo é obtido aplicando o operador de evolução temporal no estado de referência independente do tempo

$$
|t\rangle=e^{-\widehat{\kappa}(t)}\left|t_{0}\right\rangle
$$

O operador densidade dependente do tempo tem como valor esperado a expressão

$$
\rho(\boldsymbol{r}, t)=\langle t|\hat{\rho}(\boldsymbol{r})| t\rangle=\left\langle t_{0}\left|e^{\widehat{\kappa}(t)} \hat{\rho}(\boldsymbol{r}) e^{-\widehat{\kappa}(t)}\right| t_{0}\right\rangle
$$

onde $\hat{\rho}(\boldsymbol{r})=\sum_{p q} \varphi_{p}^{*}(\boldsymbol{r}) \varphi_{q}(\boldsymbol{r}) \hat{E}_{p q}, \hat{E}_{p q}=\sum_{\sigma} a_{p \sigma}^{\dagger} a_{q \sigma}$ e $a_{r \sigma}^{\dagger}$ e $a_{s \sigma}$ são, respectivamente, os operadores de criação e aniquilação dos spin-orbitais $\varphi_{p}$ e $\varphi_{q}$ com valor de spin de $\sigma$.

Na ausência da perturbação, o operador anti-Hermitiano $\hat{\kappa}(t)$ fornece um fator de fase que é anulado ao obter o valor esperado da densidade. Por outro lado, o operador $\hat{\kappa}(t)$ pode ser expandido em diferentes ordens na presença de uma perturbação

$$
\hat{\kappa}(t)=\hat{\kappa}^{(1)}(t)+\hat{\kappa}^{(2)}(t)+\hat{\kappa}^{(3)}(t)+\cdots
$$

Expandindo a equação (51) com a fórmula de Baker-Campbell-Hausdorff, substituindo a equação (52) e agrupando os termos de acordo com a ordem da expansão temos

$$
\begin{gathered}
\rho(\boldsymbol{r}, t)=\rho\left(\boldsymbol{r}, t_{0}\right)+\rho^{(1)}(\boldsymbol{r}, t)+\rho^{(2)}(\boldsymbol{r}, t)+\rho^{(3)}(\boldsymbol{r}, t)+\cdots \\
\rho\left(\boldsymbol{r}, t_{0}\right)=\sum_{p q} \phi_{p}^{*}(\boldsymbol{r}) \phi_{q}(\boldsymbol{r})\left\langle t_{0}\left|\hat{E}_{p q}\right| t_{0}\right\rangle \\
\rho^{(1)}(\boldsymbol{r}, t)=\sum_{p q} \phi_{p}^{*}(\boldsymbol{r}) \phi_{q}(\boldsymbol{r})\left\langle t_{0}\left|\left[\hat{\kappa}^{(1)}(t), \hat{E}_{p q}\right]\right| t_{0}\right\rangle \\
\rho^{(2)}(\boldsymbol{r}, t)=\sum_{p q} \phi_{p}^{*}(\boldsymbol{r}) \phi_{q}(\boldsymbol{r})\left\{\left\langle t_{0}\left|\left[\hat{\kappa}^{(2)}(t), \hat{E}_{p q}\right]\right| t_{0}\right\rangle\right. \\
\left.+\frac{1}{2}\left\langle t_{0}\left|\left[\hat{\kappa}^{(1)}(t),\left[\hat{\kappa}^{(1)}(t), \hat{E}_{p q}\right]\right]\right| t_{0}\right\rangle\right\}
\end{gathered}
$$


As correções da densidade entram no cálculo SCF como correções do termo de dois elétrons e como correção do termo de troca-correlação.

Portanto, para obter a correção de ordem $n$ da densidade dependente do tempo, temos que encontrar o valor de $\hat{\kappa}$ até a ordem $n$. Para isso, iremos utilizar o método de Ehrenfest para um operador $\hat{Q}(t)=e^{-\widehat{\kappa}(t)} \hat{Q} e^{\widehat{\kappa}(t)}$

$$
\begin{gathered}
\left\langle t\left|i \dot{\hat{Q}}(t)+\left[\hat{Q}(t), \widehat{H}_{0}(t)+\hat{V}(t)\right]\right| t\right\rangle=0 \\
\left\langle t_{0}\left|\left[\hat{Q}, e^{\widehat{\kappa}(t)}\left(\widehat{H}_{0}(t)+\widehat{V}(t)-i \frac{d}{d t}\right) e^{-\widehat{\kappa}(t)}\right]\right| t_{0}\right\rangle=0
\end{gathered}
$$

onde $\widehat{H}_{0}(t)$ é o hamiltoniano do sistema não perturbado e $\widehat{V}(t)$ descreve as interações do sistema com um campo eletromagnético. Assumindo $\hat{Q}$ como operador de energia de excitação $\widehat{E}_{p q}$ e agrupando-os num operador no formato coluna $\widehat{\boldsymbol{q}}=\left\{\widehat{E}_{p q}\right\}$, encontra-se um conjunto de equações não lineares que torna possível obter $\hat{\kappa}(t)$ na representação de frequências $\hat{\kappa}^{\omega}$. Conhecendo os valores de $\hat{\kappa}^{\omega}$ as funções resposta TD-DFT são obtidas para o operador $\hat{A}$ sujeito a perturbações periódicas de $\widehat{B}, \hat{C}, \ldots$ associadas as frequências $\omega_{1}, \omega_{2}, \ldots$.como

$$
\begin{gathered}
\langle\langle\hat{A} ; \hat{B}\rangle\rangle_{\omega_{1}}=\left\langle t_{0}\left|\left[\hat{\kappa}^{\omega_{1}}, \hat{A}\right]\right| t_{0}\right\rangle \\
\langle\langle\hat{A} ; \hat{B}, \hat{C}\rangle\rangle_{\omega_{1}, \omega_{2}}=\left\langle t_{0}\left|\left[\hat{\kappa}^{\omega_{1}, \omega_{2}}, \hat{A}\right]\right| t_{0}\right\rangle+\mathbb{P}_{1,2}\left\langle t_{0}\left|\left[\hat{\kappa}^{\omega_{1}},\left[\hat{\kappa}^{\omega_{2}}, \hat{A}\right]\right]\right| t_{0}\right\rangle
\end{gathered}
$$

A demonstração completa das funções resposta no formalismo TD-DFT estão apresentadas no livro [50].

Para obter a resposta linear, somente os termos de primeira ordem são considerados, para a resposta quadrática considera-se os termos até segunda ordem e assim sucessivamente. A teoria TD-DFT é utilizada para obter o valor esperado do operador $\hat{A}$

$$
\begin{gathered}
\langle\Psi(t)|\hat{A}| \Psi(t)\rangle=\langle\hat{A}\rangle^{(0)}+\langle\hat{A}\rangle^{(1)}+\langle\hat{A}\rangle^{(2)}+\langle\hat{A}\rangle^{(3)}+\cdots \\
\langle\hat{A}\rangle^{(1)}=\sum_{\omega_{1}}\langle\langle\hat{A} ; \hat{B}\rangle\rangle_{\omega_{1}} e^{-i \omega_{1} t} \\
\langle\hat{A}\rangle^{(2)}=\sum_{\omega_{1}, \omega_{2}}\langle\langle\hat{A} ; \hat{B}, \hat{C}\rangle\rangle_{\omega_{1}, \omega_{2}} e^{-i\left(\omega_{1}+\omega_{2}\right) t}
\end{gathered}
$$


Da mesma forma que apresentado na seção 2.2, onde as funções respostas foram obtidas de forma exata, obtemos as contribuições de ordem superior do valor esperado do momento de dipolo utilizando a função de onda TD-DFT

$$
\begin{gathered}
\lim _{\omega \rightarrow \omega_{f 0}}\left(\omega-\omega_{0 f}\right)\langle\langle\hat{A} ; \hat{B}\rangle\rangle_{\omega_{1}}=\langle 0|\hat{A}| f\rangle\langle f|\hat{B}| 0\rangle \\
\lim _{\omega_{2} \rightarrow \omega_{f 0}}\left(\omega_{2}-\omega_{f 0}\right)\langle\langle\hat{A} ; \hat{B}, \hat{C}\rangle\rangle_{\omega_{1}, \omega_{2}}=-P_{12}\left\{\sum_{n} \frac{\langle 0|\hat{A}| n\rangle\langle n|\hat{B}| 0\rangle}{-\omega_{1}+\omega_{n f}}\right\}\langle f|\hat{C}| 0\rangle
\end{gathered}
$$

Ao assumir os operadores $\hat{A}, \hat{B}$ e $\hat{C}$ como o operador de momento de dipolo $\mu_{\alpha}$ obtemos os momentos de transição linear (equação (61)), relacionado com o processo de A1F, e não linear (equação (62)), relacionado com o processo de A2F, entre os estados inicial $|0\rangle$ e final $|f\rangle$.

Na Tabela 1 apresentamos os processos ópticos não lineares mais comuns e na Tabela 2 as propriedades moleculares descritas pelas respostas de primeira, segunda e terceira ordem.

Tabela 1. Processos ópticos não lineares frequentemente analisados. Adaptado da referência [50].

\begin{tabular}{|lc|}
\hline \multicolumn{1}{|c|}{ Processos de segunda ordem } & Frequências \\
\hline Estático & $0 ; 0,0$ \\
Efeito Pockels eletro-óptico & $-\omega ; \omega, 0$ \\
Geração do segundo harmônico & $-2 \omega ; \omega, \omega$ \\
\hline \multicolumn{1}{|c|}{ Processos de terceira ordem } & Frequências \\
\hline Efeito Kerr eletro-óptico & $-\omega ; \omega, 0,0$ \\
Absorção de dois fótons & $-\omega ; \omega,-\omega, \omega$ \\
Geração de segundo harmônico induzido por & $-2 \omega ; \omega, \omega, 0$ \\
campo elétrico & $-3 \omega ; \omega, \omega, \omega$ \\
Geração de terceiro harmônico & \\
\hline
\end{tabular}


Tabela 2. Resultados obtidos utilizando funções respostas de primeira, segunda e terceira ordem e seus respectivos resíduos. Adaptado da referência [50].

\begin{tabular}{|c|c|c|}
\hline Resposta & Resíduo & Resultado \\
\hline$\alpha(-\omega ; \omega)$ & 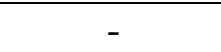 & Polarizabilidade linear. \\
\hline$\langle\langle\hat{A} ; \hat{B}\rangle\rangle_{\omega}$ & $\omega=\omega_{f 0}$ & $\begin{array}{l}\text { Elementos da matriz de transição de um fóton } \\
\text { entre os estados fundamental e excitado } f \text {. }\end{array}$ \\
\hline$\beta\left(-\omega_{\sigma} ; \omega_{1}, \omega_{2}\right)$ & - & Hiperpolarizabilidade de primeira ordem. \\
\hline \multirow[t]{3}{*}{$\langle\langle\hat{A} ; \hat{B}, \hat{C}\rangle\rangle_{(}$} & $\omega_{2}=\omega_{f 0}$ & $\begin{array}{l}\text { Elementos da matriz de transição de dois fótons } \\
\text { entre o estado fundamental excitado } f \text {. }\end{array}$ \\
\hline & $\begin{array}{l}\omega_{1}=-\omega_{f 0} \\
\omega_{2}=\omega_{g 0}\end{array}$ & $\begin{array}{l}\text { Elementos da matriz de transição de um fóton } \\
\text { entre estados excitados } f \text { e } g \text {. }\end{array}$ \\
\hline & $\begin{array}{l}\omega_{1}=-\omega_{f 0} \\
\omega_{2}=\omega_{f 0}\end{array}$ & $\begin{array}{l}\text { Momento de dipolo permanente do estado } \\
\text { excitado } f \text {. }\end{array}$ \\
\hline$\gamma\left(-\omega_{\sigma} ; \omega_{1}, \omega_{2}, \omega_{3}\right)$ & - & Hiperpolarizabilidade de segunda ordem. \\
\hline \multirow[t]{3}{*}{$\langle\langle\hat{A} ; \hat{B}, \hat{C}, \widehat{D}\rangle\rangle_{\omega_{1}, \omega_{2}, \omega_{3}}$} & $\omega_{3}=\omega_{f 0}$ & $\begin{array}{l}\text { Elementos da matriz de transição de três fótons } \\
\text { entre o estado fundamental e excitado } f \text {. }\end{array}$ \\
\hline & $\begin{array}{l}\omega_{2}=-\omega_{f 0} \\
\omega_{3}=\omega_{g 0}\end{array}$ & $\begin{array}{l}\text { Elementos de matriz de transição de dois fótons } \\
\text { entre estados excitados } f \text { e } g \text {. }\end{array}$ \\
\hline & $\begin{array}{l}\omega_{2}=-\omega_{f 0} \\
\omega_{3}=\omega_{f 0}\end{array}$ & Polarizabilidade linear do estado excitado $f$. \\
\hline
\end{tabular}

\subsubsection{Campo finito}

Quando um campo elétrico externo estático é aplicado em uma molécula, suas cargas são polarizadas. A nova configuração de menor energia na presença do campo estático resulta em uma distribuição eletrônica induzindo variações no momento de dipolo molecular. Essa técnica pode ser utilizada para obter as (hiper)polarizabilidades como apresentadas na equação (8) utilizando derivadas da energia total.

Do ponto de vista computacional, a função de onda que descreve esse sistema perturbado tem caráter estacionário, portanto, é possível determinar a energia molecular como o valor esperado de um Hamiltoniano independente do tempo. Podemos então relacionar as variações dessa energia com a expansão da polarização 


$$
\begin{aligned}
& \mu_{\alpha}^{0}=-\left.\frac{\partial \varepsilon(E)}{\partial E_{\alpha}}\right|_{E=0} \\
& \alpha_{\alpha \beta}=-\left.\frac{\partial^{2} \varepsilon(E)}{\partial E_{\alpha} \partial E_{\beta}}\right|_{E=0} \\
& \beta_{\alpha \beta \gamma}=-\left.\frac{\partial^{3} \varepsilon(E)}{\partial E_{\alpha} \partial E_{\beta} \partial E_{\gamma}}\right|_{E=0} \\
& \gamma_{\alpha \beta \gamma \delta}=-\left.\frac{\partial^{4} \varepsilon(E)}{\partial E_{\alpha} \partial E_{\beta} \partial E_{\gamma} \partial E_{\delta}}\right|_{E=0}
\end{aligned}
$$

onde $\varepsilon(E)$ são as energias $\varepsilon$ na presença do campo estático $E$. Essas derivações podem ser obtidas analiticamente ou numericamente. As derivadas numéricas são conhecidas na literatura como método de campo finito. Do ponto de vista computacional é necessário modificar somente o termo de interação de um elétron para incluir a energia de interação dipolar e calcular a energia total do sistema para diferentes valores de intensidade do campo elétrico. Dessa forma, utilizando diferenciação numérica, é possível obter os valores da polarizabilidade e das hiperpolarizabilidades moleculares.

Para ilustrar a ideia desse método vamos considerar uma molécula diatômica homonuclear, a qual tem momento de dipolo permanente nulo. A presença de um campo eletrostático externo induz forças elétricas levando a uma distribuição eletrônica assimétrica. Dessa assimetria da carga resulta um momento de dipolo. Este comportamento é associado a variações da energia $\varepsilon$ e pode ser utilizado para obter as (hiper)polarizabilidades de sistemas moleculares.

\subsection{Modelagem de líquidos moleculares}

As propriedades físico-química de substâncias representam o modo e a intensidade das interações intramoleculares e intermoleculares. As fases sólidas, líquidas e gasosas são resultados das interações intermoleculares, ocasionando alterações em propriedades físicoquímicas. Em fase gasosa, devido à baixa densidade, estas interações são fracas e podemos, frequentemente, considerar que a substância está isolada e livre no espaço. Este é o modelo do gás ideal. Enquanto em fase sólida esta interação intermolecular é intensa, caracterizada pela alta densidade, e aprisiona a substância numa determinada região. Nestas condições também é possível considerar um modelo para um sólido ideal. 
$\mathrm{Na}$ fase líquida, as moléculas interagem fortemente e não estão fixas no espaço. Isto está relacionado com a densidade e o livre caminho médio, os quais assumem valores intermediários entre aqueles do sólido e do gás. Devido a essa complexidade, não existe um modelo de líquido ideal e métodos capazes de descrever as interações presentes nesse ambiente são mais complexos.

Idealmente, incluir muitas moléculas de solvente de forma explícita nos cálculos de estrutura eletrônica é uma forma realista de computar as interações soluto-solvente, porém o custo computacional torna-se elevado impossibilitando tais estudos. Para viabilizar o custo computacional nesses estudos podemos utilizar modelos simplificados para representar o solvente. Nos chamados modelos contínuos, os solventes perdem a estrutura molecular e suas propriedades macroscópicas são utilizadas para descrever as interações soluto-solvente. Assim, o soluto é inserido em uma cavidade de vácuo dentro deste meio contínuo, dielétrico ou condutor, e sofre uma polarização devido aos efeitos eletrostáticos deste meio. Decorrente dessa perda de estruturação das moléculas de solvente as interações específicas, tal como ligações de hidrogênio, são negligenciadas.

Incluir as moléculas de solvente explicitamente nos cálculos de estrutura eletrônica do soluto é uma forma mais realista de computar as interações soluto-solvente, porém, além de aumentar expressivamente o tempo de computação as flutuações termodinâmicas nem sempre estão incluídas. A modelagem molecular utilizando campos de força parametrizados para representar as interações intra e intermoleculares reproduzem o movimento atômico/molecular utilizando representações clássicas da carga, do raio atômico, da distância de ligação etc. Dessa forma é possível obter parâmetros estruturais (como quantidade de moléculas de solvente que realizam ligações de hidrogênio) e macroscópicos (como a taxa de difusão), porém nenhuma informação de propriedades eletrônicas pode ser obtida.

Para equilibrar o custo computacional ao descrever moléculas solvatadas, uma das metodologias mais utilizadas atualmente combina cálculos de estrutura eletrônica com cálculos utilizando campos de força parametrizados. Assim, a região de maior interesse é descrita com o viés da mecânica quântica, enquanto a região periférica do sistema é descrita utilizando a mecânica molecular clássica; esta metodologia é conhecida por Quantum Mechanics / Molecular Mechanics (QM/MM) [86]. Para evitar a parametrização dos campos de força clássicos, podemos calcular as interações utilizando métodos de estrutura eletrônica, contudo o alto custo computacional limita a quantidade de moléculas a serem estudadas. 
Outra proposta para descrever as propriedades eletrônicas de moléculas solvatadas envolve uma abordagem em duas etapas. Na primeira etapa a simulação molecular é realizada (utilizando métodos de estrutura eletrônica ou campos de força clássicos) incluindo efeitos termodinâmicos e em seguida os cálculos de propriedades eletrônicas são realizados em configurações selecionadas da simulação. Este método é conhecido como Sequential-QM/MM (S-QM/MM) [87,88] e foi desenvolvido em nosso grupo de pesquisa.

A seguir, apresentamos aspectos e limitações das técnicas de modelagem de líquidos moleculares utilizadas neste trabalho. Novamente, a descrição apresentada a seguir é sucinta e não pretende substituir as já renomadas referências que serão citadas no decorrer das subseções.

\subsubsection{Modelos contínuos}

No início da década de 1930 surgiram os trabalhos pioneiros de Kirkwood [89] e Onsager [90] que incluíam um meio contínuo para representar os efeitos de líquidos em cálculos de propriedades eletrônicas. Este meio contínuo representava um material dielétrico com a constante dielétrica do respectivo líquido de interesse. O soluto é inserido dentro de uma cavidade esférica de raio $a$ (chamado raio de Onsager, ver lado esquerdo da Figura 4). O próprio autor salienta a dificuldade relacionada à escolha apropriada do valor de $a$. Para melhorar a descrição das interações soluto-solvente incluiu-se um campo de reação auto consistente $[91,92]$ e transformou-se a única cavidade esférica em uma superposição de cavidades esféricas centradas nos núcleos atômicos do soluto (ver lado direito da Figura 4). Tipicamente a cavidade de cada átomo é $20 \%$ maior que o seu raio de Van der Waals. Propôs-se então um ambiente dielétrico polarizável, conhecido por PCM [93], o qual é largamente utilizado nos dias atuais. Para maiores detalhamentos destes métodos recomenda-se o artigo de revisão elaborado por Tomasi e coautores [94] onde os autores apresentam as teorias e as características dos modelos de solventes contínuos utilizados em conjunto com a mecânica quântica.

Uma vez que não são incluídos elétrons adicionais devido ao solvente, a grande vantagem dos métodos contínuos é o custo computacional, que é geralmente apenas um pouco maior que o cálculo do sistema isolado. Estes modelos geralmente apresentam bons resultados quando as interações eletrostáticas são dominantes. Como desvantagem evidencia-se a escolha do modelo de cavitação, a ausência de interações específicas como ligações de hidrogênio e a ausência de efeitos térmicos. 

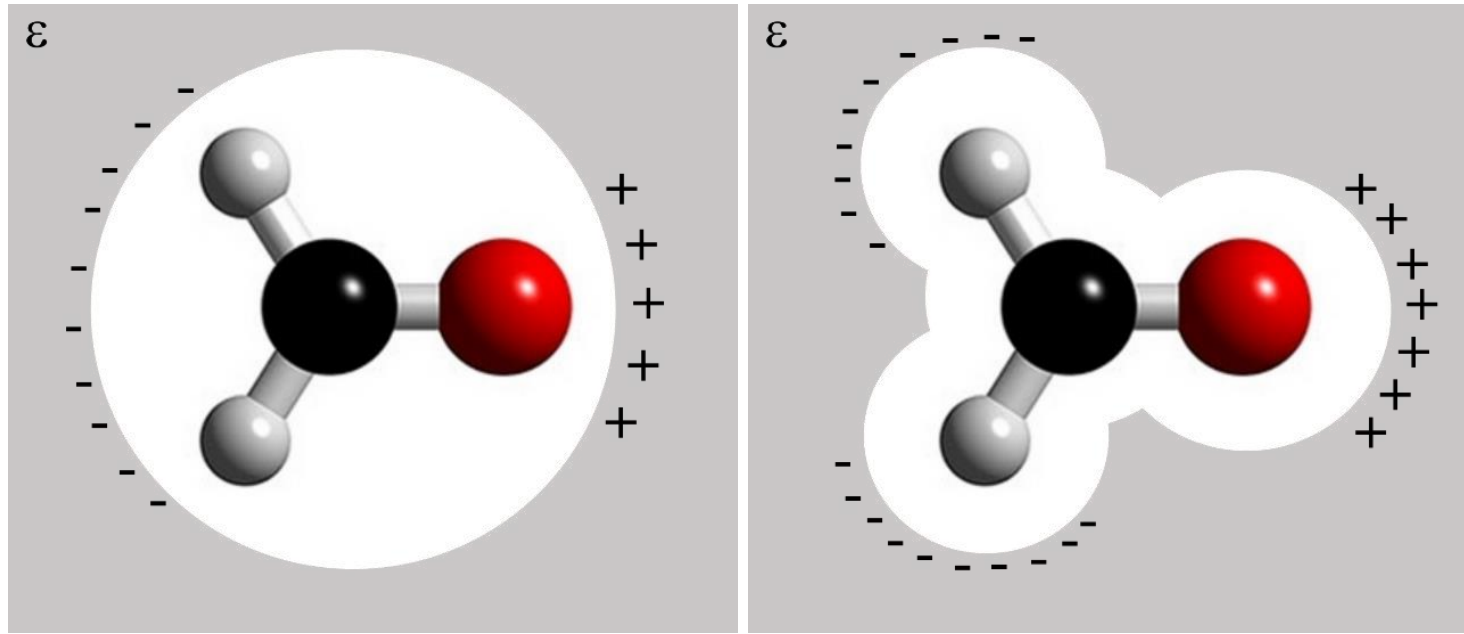

Figura 4. Modelo de cavitação baseado em uma única esfera (lado esquerdo) e na superposição de esferas (lado direito) centradas em cada átomo.

\subsubsection{Amostragem de Monte Carlo Metropolis}

Com a amostragem de Monte Carlo Metropolis (MC) tornou-se possível obter propriedades físicas médias sobre configurações geradas de forma estocástica. Esta abordagem não determinística evita a integração das equações de movimento e com isso é possível encontrar descrições equivalentes as dinâmicas moleculares com um menor custo computacional. Contudo esta abordagem não é adequada para análises envolvendo o movimento atômico em função do tempo, como a reação de transferência de próton por exemplo.

O Método de Monte Carlo Metropolis [95] propõe transformar integrais em médias sobre $N_{\text {tot }}$ valores aleatórios gerados para qualquer distribuição $\rho(x)$, apresentando grande vantagem computacional. Considerando a $i$-ésima configuração gerada como $\xi_{i}$, a relação entre a integral e a média é dada por

$$
F=\int_{a}^{b} d x f(x)=\int_{a}^{b} d x\left(\frac{f(x)}{\rho(x)}\right) \rho(x)=\left\langle\frac{f\left(\xi_{i}\right)}{\rho\left(\xi_{i}\right)}\right\rangle_{N_{t o t}} .
$$

Utilizando a integração apresentada na equação (64) e sendo $\rho(x)$ arbitrário, Metropolis sugeriu uma amostragem preferencial $\rho(x)=\rho_{N V T}$ para aumentar a eficiência computacional. Baseada na seguinte argumentação: Sendo $f(r)$ modulada pela distribuição $\rho_{N V T}=e^{-\beta U(\boldsymbol{r})}$, é mais eficiente realizar a amostragem onde $\rho_{N V T} \neq 0$, onde $\beta=1 / k_{B} T, k_{B}$ é a constante de Boltzmann, T é a temperatura e $U(\boldsymbol{r})$ é a energia potencial em função das coordenadas $\boldsymbol{r}$. As configurações são geradas aleatoriamente e a energia do sistema alterado é o critério de aceitação da nova configuração. Sempre que a energia do novo estado for menor que a do estado 
anterior a configuração é aceita, caso contrário é feito um sorteio aleatório para aceitar ou não o novo estado.

As novas configurações são geradas considerando tentativas de translações com deslocamentos $\delta t$ e de rotações $\delta \theta$ (mantendo as coordenadas na representação cartesiana e definindo um eixo sobre a molécula). Quando a simulação é realizada considerando a pressão constante (ensemble NPT) tentativas de alterar o tamanho $L$ da caixa também são realizadas. Resumidamente, o método MC segue o seguinte algoritmo para mudar de um estado $i$ para outro estado $j$. (1) Calcular $U_{i}$; (2) Transladar e/ou rotacionar uma molécula de $\delta r$ e/ou $\delta \theta$ e aumentar ou diminuir o tamanho da caixa de $\delta L$; (3) Calcular $U_{j}$; (4) Aplicar os critérios de aceitação da nova configuração; (5) Redefinir $U_{i}=U_{j}$ caso a nova configuração seja aceita; e (6) voltar ao passo 2 até atingir um número predefinido de ciclos.

Neste trabalho, as simulações utilizando o algoritmo de MC foram realizadas no programa DICE [96,97]. As moléculas foram consideradas rígidas e livres para serem transladadas e rotacionadas dentro de uma caixa cúbica com condições periódicas de contorno. O potencial de interação $U_{i j}$ entre dois sítios atômicos $i$ e $j$ de diferentes moléculas é formado pela combinação dos potenciais de Lennard-Jones e de Coulomb e está representado pela equação (65). Estes potenciais dependem de quatro parâmetros: $\epsilon_{i j}=\sqrt{\epsilon_{i} \epsilon_{j}}-$ representa a profundidade do potencial de interação; $\sigma_{i j}=\sqrt{\sigma_{i} \sigma_{j}}$ - representa a posição no qual o potencial é zero; da carga $q$ de cada átomo e da distância $r_{i j}$ entre os dois pares. Os subíndices referemse aos sítios atômicos.

$$
U_{i j}=4 \epsilon_{i j}\left[\left(\frac{\sigma_{i j}}{r_{i j}}\right)^{12}-\left(\frac{\sigma_{i j}}{r_{i j}}\right)^{6}\right]+\frac{q_{i} q_{j}}{r_{i j}},
$$

Os valores de $\epsilon_{i}$ e de $\sigma_{i}$ das moléculas simuladas neste trabalho foram definidos pelo campo de força All Atom Optimized Potential for Liquid Simulations (OPLS-AA) [98,99] e as cargas foram estimadas com cálculos de estrutura eletrônica que ajustam os valores de carga nos núcleos atômicos para reproduzir o potencial eletrostático da molécula soluto. Este procedimento tem sido adotado e os resultados obtidos vem mostrando uma boa acurácia [100$103]$.

\subsubsection{Dinâmica molecular}

A dinâmica molecular é fundamentada na integração das equações de movimento de todos os átomos presentes na simulação. Esta abordagem tem sido largamente utilizada para 
sistemas físicos, químicos e biológicos quando as moléculas envolvidas apresentam grande flexibilidade. O conceito da evolução temporal da dinâmica consiste na solução da equação (66) para o $i$-ésimo átomo de massa $M_{i}$ e coordenadas $\boldsymbol{r}_{i}$ na presença de um potencial $U(\boldsymbol{r})$.

$$
M_{i} \ddot{\boldsymbol{r}}_{l}(t)=-\nabla_{\mathrm{i}} U(\boldsymbol{r})
$$

Como o movimento é obtido para cada átomo, a translação das moléculas e a flexibilidade intramolecular estão automaticamente garantidos. Neste sentido, nenhuma precaução extra é necessária para obter tais movimentos.

Vários algoritmos de integração das equações foram desenvolvidos como Verlet [104], Leap-Frog [105] e Velocidade de Verlet [106]. Como as velocidades dos átomos se alteram no decorrer da simulação, é necessário garantir que a temperatura desejada seja mantida. Neste sentido inclui-se os chamados termostatos. Escalonar as velocidades por um fator $\alpha=$ $\sqrt{T / T(t)}$ é uma forma simples de manter a temperatura constante. Porém a inclusão deste e de outros termostatos podem violar a conservação da energia total.

A hipótese de ergodicidade garante que a média temporal é equivalente à média sobre o ensemble devido a probabilidade de amostrar todas as configurações acessíveis. Como na dinâmica molecular realiza-se $n$ incrementos de $\delta t$ e a posição e o momento são conhecidos em todos os instantes, a representação algébrica de propriedades médias pode ser expressa por

$$
\begin{gathered}
\bar{f}=\langle f\rangle_{\text {ensemble }} \\
\bar{f}=\frac{1}{n} \sum_{i=1}^{n} f\left(\boldsymbol{r}\left(t_{i}\right), \boldsymbol{p}\left(t_{i}\right)\right)
\end{gathered}
$$

O potencial $U(\boldsymbol{r})$ pode ser obtido utilizando uma abordagem clássica ou quântica. $\mathrm{O}$ potencial clássico contém contribuições intra e intermoleculares parametrizados e expressos por equações algébricas. As contribuições intramoleculares consideram (i) potenciais harmônicos para representar ligações químicas e ângulos de abertura formados por três átomos, (ii) potenciais periódicos para representar ângulos impróprios e ângulos torcionais formados por quatro átomos. Para os ângulos torcionais além do ângulo periódico $\varphi$ uma fase $\phi$ é considerada para deslocar a posição dos mínimos/máximos da função. As contribuições intermoleculares são representadas utilizando, frequentemente, o potencial de Lennard-Jones somado ao potencial de Coulomb. O potencial $U(\boldsymbol{r})$ é representado por 


$$
\begin{aligned}
U(\boldsymbol{r}) & =\sum_{\text {ligações }} \frac{1}{2} k_{r}\left(\boldsymbol{r}-\boldsymbol{r}_{\mathbf{0}}\right)^{2} \\
& +\sum_{\text {ângulos }} \frac{1}{2} k_{\theta}\left(\theta-\theta_{0}\right)^{2} \\
& +\sum_{\text {impróprios }} \frac{V}{2}[1+\cos (2 \varphi-180)] \\
& +\sum_{\text {Loulomb }} \frac{q_{i} q_{j}}{r_{i j}} \\
& \left.\left.+\sum_{\text {torcionais }} \frac{\sigma_{i j}}{\sum_{i j}}\right)^{12}-\left(\frac{\sigma_{i j}}{r_{i j}}\right)^{6}\right] \\
& \left.\sum_{i j}+\cos (n \varphi-\phi)\right]
\end{aligned}
$$
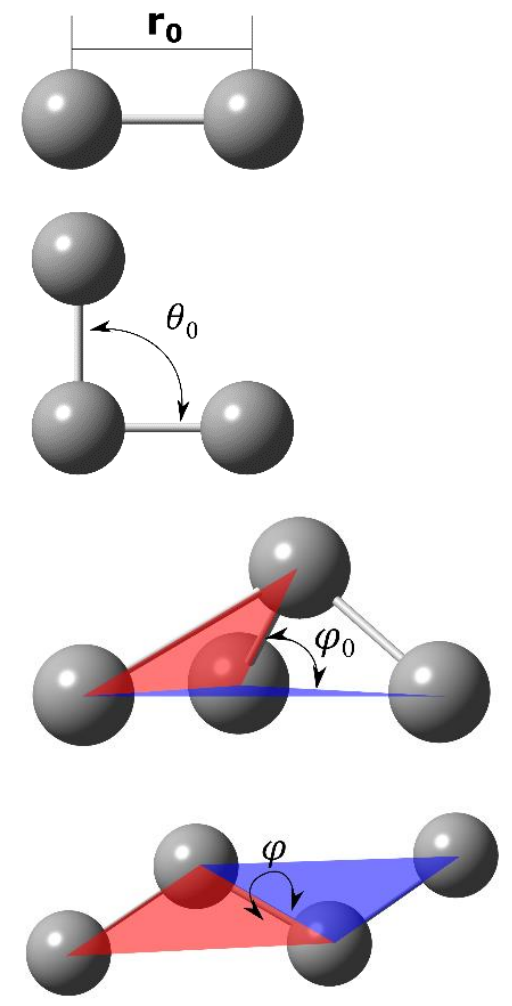

É possível obter o potencial $U(\boldsymbol{r})$ utilizando métodos de estrutura eletrônica em vez de campos de forças clássicos. Nessa situação, quando a propagação dos movimentos nucleares ocorre de forma clássica atribuímos o nome de Born-Oppenheimer Molecular Dynamics (BOMD). Nesta aproximação os núcleos são considerados partículas clássicas e a cada incremento de tempo é necessário realizar um novo cálculo de estrutura eletrônica para computar os valores do potencial $U(\boldsymbol{r})$ e consecutivamente o seu gradiente. Isso torna o custo computacional desta metodologia muito elevado e atualmente apenas centenas de átomos são simulados na escala de tempo de pico-segundos. Por outro lado, nas simulações inteiramente clássicas é possível simular milhares de átomos por microssegundos. Quando $U(\boldsymbol{r})$ é obtido com cálculos de estrutura eletrônica a propagação das posições atômicas são mais precisos que os obtidos com campos de forças parametrizados.

Devido ao alto custo computacional da BOMD, para resolução do problema eletrônico, geralmente a DFT é utilizada considerando aproximações adicionais para obter ganho de 
Tárcius N. Ramos

performance computacional. A utilização de pseudopotenciais para os elétrons internos e um conjunto de funções de ondas planas combinadas com funções gaussianas são algumas aproximações que podem ser realizadas neste sentido.

\subsubsection{Metodologia Sequential - Quantum Mechanics / Molecular Mechanics}

Considerar um líquido molecular, centenas ou milhares de átomos, utilizando métodos de estrutura eletrônica é custoso computacionalmente, por outro lado uma aproximação totalmente clássica não é suficiente para descrever as propriedades eletrônicas. Para tornar este problema factível surgiram os métodos híbridos, que dividem o sistema em duas partes, sendo elas: a região quântica descrevendo a estrutura eletrônica dos sítios atômicos de maior interesse e a região clássica para computar os efeitos de longo alcance. O método S-QM/MM [87,88] foi desenvolvido para separar as etapas clássicas e quânticas, propondo a realização de uma simulação completamente clássica seguida de cálculos quânticos independentes em configurações extraídas da simulação. Para obter valores representativos de propriedades eletrônicas médias é necessário garantir a convergência estatística, sendo que para aumentar a eficiência da convergência das médias os cálculos são realizados sobre configurações estatisticamente descorrelacionadas. Contudo, a correlação estatística somente é calculada ao término da simulação, assim, essa separação em duas etapas evita que cálculos quânticos em configurações correlacionadas sejam realizados e torna esta abordagem computacional mais eficiente. Com esta abordagem os resultados finais podem ser obtidos como médias ou valores mais prováveis, além de se poder analisar a convergência estatística destes valores [87,88]. O fluxograma do método S-QM/MM pode ser observado na Figura 5.

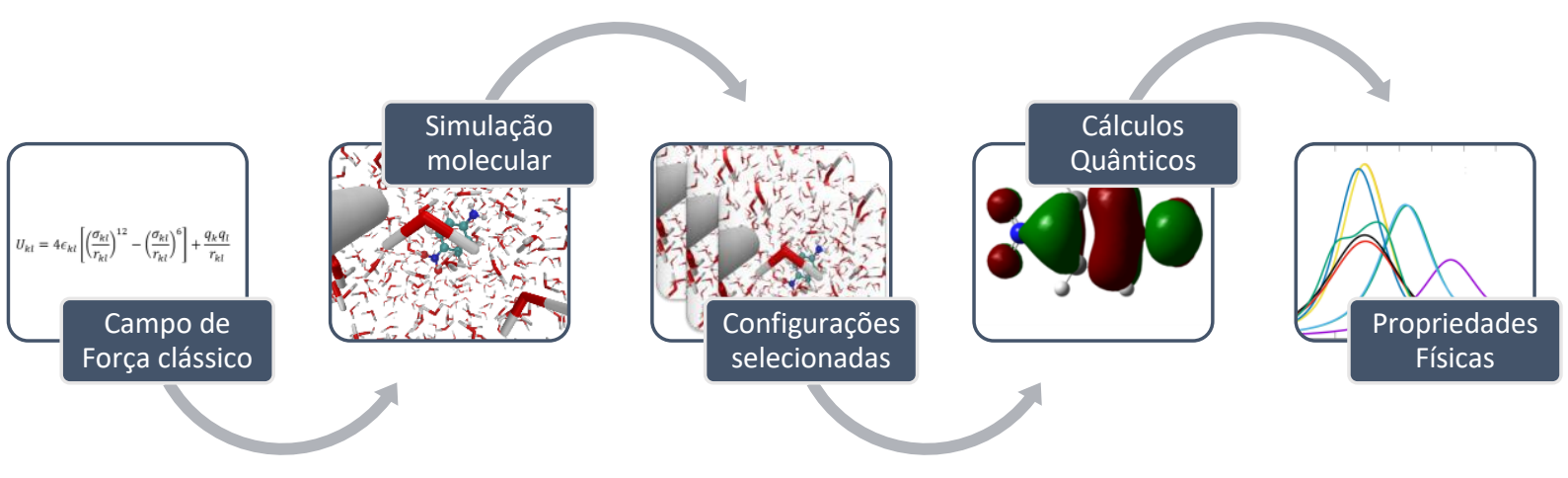

Figura 5. Fluxograma indicando a sequência de cálculos realizados no método S-QM/MM.

Uma proposta eficiente para de se obter as contribuições puramente eletrostática dos efeitos de solventes em uma simulação sequencial foi desenvolvida em nosso grupo de 
pesquisa, o Average Solvent Electrostatic Configuration (ASEC) [107]. Nesta aproximação todos os sítios do solvente incluídos nos cálculos de estrutura eletrônica são considerados como cargas pontuais. Ao invés de realizar um cálculo quântico para cada configuração descorrelacionada selecionada, com o ASEC obtemos o valor equivalente em um único cálculo. No ASEC as configurações são sobrepostas e a carga de cada sítio do solvente é normalizada pela quantidade de configurações utilizadas.

\subsection{Quantificação da transferência de carga durante excitação}

O processo de absorção de dois fótons geralmente é favorecido em transições com alta transferência de carga. Analisar as cargas eletrônicas sobre os sítios atômicos dos estados fundamental e excitado pode não ser a forma mais adequada de computar uma possível transferência de carga pois podem existir densidades delocalizadas. Por outro lado, uma simples análise do mapa das densidades eletrônicas (densidade em cores indicando as cargas positivas e negativas) dos estados envolvidos pode não ser trivial. Uma forma de quantificar a transferência de carga foi proposta analisando a diferença entre os potenciais eletrostáticos do estado fundamental e do estado excitado [108,109]. Neste sentido, propõe-se quatro pontos para realizar essa análise:

- Definição da carga transferida pela integração da função de diminuição da densidade sobre todo o espaço;

- Definição dos baricentros de diminuição e incremento da densidade;

- Definição do comprimento de transferência de carga como a distância entre os baricentros;

- Cálculo do momento de dipolo associado a esta transferência.

Definindo duas funções que representam a diferença positiva $\rho_{+}(r)$ e negativa $\rho_{-}(r)$ entre as densidades eletrônicas do estado excitado $\left(\rho_{E X}\right)$ e fundamental $\left(\rho_{G S}\right)$ como

$$
\begin{gathered}
\Delta \rho(r)=\rho_{E X}-\rho_{G S} \\
\rho_{+}(r)=\left\{\begin{array}{c}
\Delta \rho(r) \text { se } \Delta \rho(r)>0 \\
0 \text { se } \Delta \rho(r)<0
\end{array}\right. \\
\rho_{-}(r)=\left\{\begin{array}{c}
\Delta \rho(r) \text { se } \Delta \rho(r)<0 \\
0 \text { se } \Delta \rho(r)+0
\end{array}\right.
\end{gathered}
$$


A quantidade de carga transferida é obtida integrando uma das duas densidades definidas na equação (69), observando a inversão do sinal entre elas

$$
q_{t}=\int \rho_{+}(r) d r=-\int \rho_{-}(r) d r
$$

Com estas duas funções (equação (69)) os vetores dos baricentros são facilmente obtidos integrando as densidades e posições em todo o espaço

$$
B_{ \pm}(r)=\frac{\int r \rho_{ \pm}(r) d r}{\int \rho_{ \pm}(r) d r}
$$

O deslocamento da carga e é definido como a distância entre os baricentros

$$
R=\left|B_{+}-B_{-}\right|
$$

A diferença entre o momento de dipolo do estado fundamental e excitado é calculado como o produto do deslocamento da carga com a quantidade de carga transferida

$$
|\Delta \mu|=\left|\mu_{E X}-\mu_{G S}\right|=R q_{t}
$$

Este procedimento pode ser utilizado em qualquer nível de cálculo desde que as densidades dos estados fundamental e excitado sejam factíveis. Para calcular o índice de transferência de carga utilizamos um código escrito por Jacquemin [110]. 


\section{Capítulo 3 RESULTADOS}

\subsection{Alargamento de linha espectral para simulação da seção de choque de absorção de dois fótons da para-Nitroanilina em meio líquido}

\subsubsection{Introdução}

Utilizando perturbação dependente do tempo de segunda ordem é possível reduzir a demanda computacional e empregar métodos de química quântica mais rigorosos como Coupled Cluster ou TD-DFT para obter as probabilidades de transição de A2F. Porém, um parâmetro adicional e importante está relacionado com o alargamento espectral. Este parâmetro é essencial para simular a seção de choque de A2F. Frequentemente a função Lorentziana é utilizada para representar esse alargamento espectral de transições eletrônicas e ela depende da largura de linha completa a meia altura $(\Gamma)$. Portanto o valor de $\Gamma$ influencia o valor da seção de choque de A2F. Contudo não existe um consenso em como definir o valor de $\Gamma$. Essa dificuldade em atribuir o valor de $\Gamma$ não é peculiar a obtenção teórica da seção de choque de A2F e uma dependência similar também está presente na seção de choque de A1F e da absortividade molar.

Em cálculos de seção de choque de A2F, frequentemente o valor de $0.1 \mathrm{eV}$ é atribuído de forma arbitrária. Algumas tentativas têm sido realizadas para descrever $\Gamma$ como uma estimativa da variação da energia de transição [41] quando um conjunto de configurações é extraído de simulações moleculares. Uma recente alternativa para reproduzir o formato assimétrico de bandas de absorção combina o alargamento homogêneo proveniente de espectros de $\mathrm{A} 2 \mathrm{~F}$ resolvidos vibracionalmente com a contribuição não homogênea proveniente da variação da energia de transição obtida de simulações moleculares realizados com geometrias rígidas [111]. Contudo, se um valor experimental de $\Gamma$ é conhecido, é possível utilizar essa informação para construir o espectro teórico para o composto isolado e utilizando a aproximação PCM para incluir os efeitos de solventes. Neste trabalho, nós incluímos moléculas de solvente de forma explícita provenientes de configurações extraídas de simulações moleculares. Obtivemos os valores máximos da seção de choque de A2F utilizando cinco formas diferentes de convoluir o espectro, essas formas envolvem: valores experimentais de $\Gamma$ relacionados com o espectro de A1F; valores de $\Gamma$ relacionados com o desvio padrão de energias 
de transição; e uma nova sugestão de utilizar um ajuste para atribuir o valor de $\Gamma$. Neste procedimento de ajuste, o valor de $\Gamma$ atribuído para o conjunto de configurações é tal que o valor da largura de linha do espetro total $\Gamma_{\text {tot }}$ (considerando a contribuição de todas as configurações selecionadas) reproduza um valor previamente definido.

Compostos não centrossimétricos apresentam regras de seleção flexíveis, permitindo que um estado excitado seja acessado tanto pela A1F quanto pela A2F. Considerando esta categoria de compostos, é razoável e plausível assumir o valor de $\Gamma_{\text {tot }}$ como o valor do alargamento obtido do espectro experimental de A1F. Tal aproximação permite simular a seção de choque de $\mathrm{A} 2 \mathrm{~F}$ mesmo quando não existem dados experimentais relacionados com o processo não linear. Por outro lado, esta não deve ser uma boa aproximação se o estado eletrônico de interesse não é acessível por ambos os processos (linear e não linear). O objetivo deste trabalho foi determinar a melhor forma de convoluir o espectro de A2F comparando diferentes procedimentos para atribuir o valor de $\Gamma$ utilizando a metodologia S-QM/MM. As simulações moleculares consideradas incluem modelagem clássica com geometrias rígidas utilizando o método MC e considerando a modelagem quântica com moléculas flexíveis utilizando o método BOMD.

A para-Nitroanilina (pNA) é uma molécula com grande deslocamento solvatocrômico $(0.99 \mathrm{eV})$ entre a fase gasosa e em solução aquosa [112,113]. As propriedades espectroscópicas da pNA têm sido largamente estudadas utilizando métodos de estrutura eletrônica, desde para a pNA isolada até solvatada em meios supercríticos [102,114-122]. A pNA apresenta grupos periféricos doadores e aceitadores de carga e a transição $\pi \rightarrow \pi^{*}$ de menor energia é acessível tanto por processos de A1F um quanto de A2F. Embora as descrições teóricas da energia de transição não reproduzam com boa precisão o deslocamento solvatocrômico utilizando a TDDFT, os resultados obtidos com o funcional CAM-B3LYP têm mostrado relativa concordância com métodos baseados em Coupled Cluster [59,119,120].

Estudos prévios sobre $\mathrm{A} 2 \mathrm{~F}$ da pNA foram realizados utilizando métodos semiempíricos, DFT e Coupled Cluster [120,123,124] e também incluindo efeitos vibracionais [125] e micro hidratação [126]. Os autores destes estudos reportaram valores da seção de choque de A2F entre 25.1 e 107 GM, os quais estão relativamente distantes dos 8 e 12 GM observados experimentalmente, respectivamente, em dioxano e dimetilsulfóxido [127]. É importante destacar que os diferentes valores de $\Gamma$ e de constantes presentes nas expressões dificultam comparações diretas entre valores teóricos da seção de choque de A2F. Recentemente, a seção 
de choque de A2F da pNA em diferentes líquidos foram medidas [127] abrindo possibilidades para investigações teóricas. A baixa solubilidade e o baixo sinal não linear da pNA dificultam obter o espectro de A2F em água e justificam a ausência do valor experimental da seção de choque de $\mathrm{A} 2 \mathrm{~F}$ em água.

Dessa forma, consideramos a pNA em três meios líquidos: 1,4-dioxano (chamado somente como dioxano no decorrer do texto), dimetilsulfóxido (DMSO) e água. As interações da pNA com estes solventes foram consideradas utilizando simulações moleculares com MC e com BOMD. A seção de choque de A2F também foi obtida utilizando aproximação PCM. Como veremos a seguir, o procedimento de ajuste do espectro total mostrou a melhor concordância com os resultados experimentais para a seção de choque de A2F e, além disso, também foi possível obter informações sobre a contribuição homogênea do alargamento espectral. Enfatizamos que nosso trabalho busca obter melhores descrições para a intensidade máxima da seção de choque de A2F e não em descrever detalhadamente a forma da banda de absorção.

\subsubsection{Detalhes computacionais}

Todas as geometrias da pNA foram obtidas utilizando algoritmos de minimização de energia seguidas de cálculos de segunda derivada para confirmar a condição de mínimo utilizando MP2/aug-cc-pVDZ. Os efeitos de solventes foram incluídos neste processo considerando a aproximação PCM, implementado no programa Gaussian [81], para três solventes: dioxano, DMSO e água. As escolhas dos solventes dioxano e DMSO foram devido à existência de resultados experimentais para a seção de choque de A2F [127]. Embora não exista resultados experimentais da $\mathrm{A} 2 \mathrm{~F}$ em água, a escolha deste solvente favorece comparações entre simulações moleculares clássicas e quânticas. O computacionalmente econômico método de MC (considerando campo de força clássico) e o custoso método BOMD (considerando o potencial quântico) foram utilizados para este propósito.

As simulações com moléculas rígidas foram realizadas no programa DICE [96] utilizando o algoritmo de MC. Depois das simulações, um conjunto de configurações estatisticamente descorrelacionadas [87,88] foram selecionadas para cálculos de mecânica quântica, utilizando a metodologia S-QM/MM. Esta abordagem tem mostrado relativo sucesso ao descrever diversas propriedades moleculares incluindo polarização eletrônica [128], espectros de absorção em líquidos, em ambientes complexos como água supercrítica [100-103] e também em proteína [129]. Os valores dos parâmetros de Lennard-Jones para o campo de 
força clássico da pNA foram atribuídos utilizando OPLS-AA e os valores das cargas foram atribuídos utilizando o ajuste do potencial eletrostático CHELPG obtido com MP2/aug-ccpVDZ e com a aproximação PCM para o solvente.

Os campos de forças dos solventes para as simulações moleculares foram adotados como definidos nas referências: dioxano [130], DMSO [131,132] e água (SPC/E) [133]. Caixas cúbicas com condições periódicas de contorno contendo a molécula de pNA mais N moléculas de solvente foram previamente termalizadas e adicionalmente realizamos $2.5 \times 10^{8}$ passos de MC para produzir as configurações S-QM/MM. Adotamos o número N=1000 de moléculas de solvente para as simulações em dioxano e em água e N=500 em DMSO. Todas as simulações foram realizadas no ensemble NPT em condições ambiente $(298.15 \mathrm{~K}$ de temperatura e $1 \mathrm{~atm}$ de pressão). As ligações de hidrogênio foram identificadas considerando distâncias entre os átomos doadores-aceitadores menores que $3.5 \AA$ A ângulos entre doador-aceitador-hidrogênio menores que $40^{\circ}$ e a energia de interação soluto-solvente menores que $-3.0 \mathrm{kcal} \mathrm{mol}^{-1}$ para a simulação em água e $-1.5 \mathrm{kcal} \mathrm{mol}^{-1}$ para a simulação em DMSO. Estes critérios são necessários para excluir moléculas de solvente próximas do soluto que não estão efetivamente realizando ligações de hidrogênio [134,135]. Para cada simulação extraímos um conjunto de 100 configurações estatisticamente descorrelacionadas e o submetemos aos cálculos de estrutura eletrônica para obter as transições de A2F. Também consideramos a aproximação ASEC para computar os efeitos eletrostáticos do solvente na seção de choque de A2F.

As trajetórias das simulações realizadas com BOMD da pNA isolada e em água foram previamente realizadas [121]. Elas foram analisadas neste trabalho para fornecer informações sobre o alargamento espectral da A2F e para comparar os valores da seção de choque de A2F com resultados obtidos com as simulações clássicas com MC. Duas simulações foram realizadas no ensemble NVT: a pNA isolada com os integradores Martyna e Tuckerman para condições não periódicas de contorno [136] e para a pNA com mais 96 moléculas de água em uma caixa cúbica de tamanho $\mathrm{L}=14.59 \AA$ A. A temperatura das simulações foi de $298 \mathrm{~K}$. O funcional BLYP-D3 combinado com o conjunto de funções base MOLOPT-SR [137] foi utilizado para obter a energia eletrônica durante a simulação com BOMD realizada no programa CP2K [138]. Os valores de carga elétrica - 0.786 e 0.393 u.a. foram atribuídos, respectivamente, para os átomos de oxigênio e hidrogênio da água para os cálculos considerando somente as contribuições eletrostáticas do solvente. As energias e intensidades das transições via dois fótons foram obtidas para 200 configurações selecionadas a cada 125 fs dos últimos 25 ps das trajetórias. 
Os cálculos das intensidades de transição de dois fótons foram realizados utilizando teoria de resposta quadrática desenvolvido para o método TD-DFT com o funcional CAMB3LYP como implementado no programa Dalton 2016.2 [139,140]. Adotamos o conjunto de funções base para as moléculas da seguinte forma. (i) aug-cc-pVDZ para a pNA, (ii) 6-31+G(d) para as moléculas de dioxano e de DMSO, (iii) aug-cc-pVDZ para as moléculas de água quando incluímos as somente as moléculas que realizam ligações de hidrogênio, e (iv) 6-31+G(d) para as moléculas de água quando incluímos as 15 moléculas de água mais próximas. As trajetórias das simulações realizadas com BOMD foram fornecidas pelo Prof. Benedito Cabral [121].

$\mathrm{O}$ resultado do método $\mathrm{S}-\mathrm{QM} / \mathrm{MM}$ fornece os valores de propriedades eletrônicas calculadas para as configurações extraídas de simulações moleculares. Geralmente, para construir o espectro de absorção, seja linear ou não linear, são realizados cálculos para obter as energias e intensidades de transição e, também, é necessário considerar um alargamento espectral. A seção de choque de A2F (descrita pela equação (43)) considera apenas o resultado de uma configuração e para combinar os resultados de dezenas (ou até mesmo centenas) de configurações consideramos cinco procedimentos para obter o espectro de A2F da pNA em solventes. Os procedimentos são identificados por Pn onde n=1,2,3,4 e 5, são eles:

- P1 - considera a energia $\overline{\omega_{0 f}}$ e a intensidade $\overline{\omega^{2} \delta_{0 f}}$ média de cada estado excitado assumindo único valor de $\Gamma$, geralmente $\Gamma=\Gamma_{\text {exp }}$;

- $\mathrm{P} 2$ - considera a energia $\overline{\omega_{0 f}}$ e a intensidade $\overline{\omega^{2} \delta_{0 f}}$ média de cada estado excitado assumindo um valor de $\Gamma$ para cada estado excitado como o desvio padrão da energia de transição $\Gamma=\Gamma_{s t d}=\sqrt{8 \ln 2} \times \sigma_{s t d}\left(\omega_{0 f}\right)$;

- P3 - obtém o espectro médio considerando a convolução para cada configuração assumindo único valor de $\Gamma$, geralmente $\Gamma=\Gamma_{\text {exp }}$;

- P4 - obtém o espectro médio considerando a convolução para cada configuração onde cada estado excitado terá o valor de $\Gamma=\Gamma_{s t d}=\sqrt{8 \ln 2} \times \sigma_{s t d}\left(\omega_{0 f}\right)$;

- P5 - obtém o espectro médio considerando a convolução para cada configuração com $\Gamma=\Gamma_{\text {fit }}$ para todas as transições. $\mathrm{O}$ valor de $\Gamma_{\text {fit }}$ é ajustado para que a largura do espectro total $\left(\Gamma_{t o t}\right)$ seja igual ao valor dado da largura $\Gamma$, geralmente $\Gamma_{\text {tot }}=\Gamma_{\text {exp }}$.

Na Figura 6 apresentamos uma ilustração para facilitar a visualização dos efeitos dos diferentes procedimentos na largura de linha do espectro total simulado. 


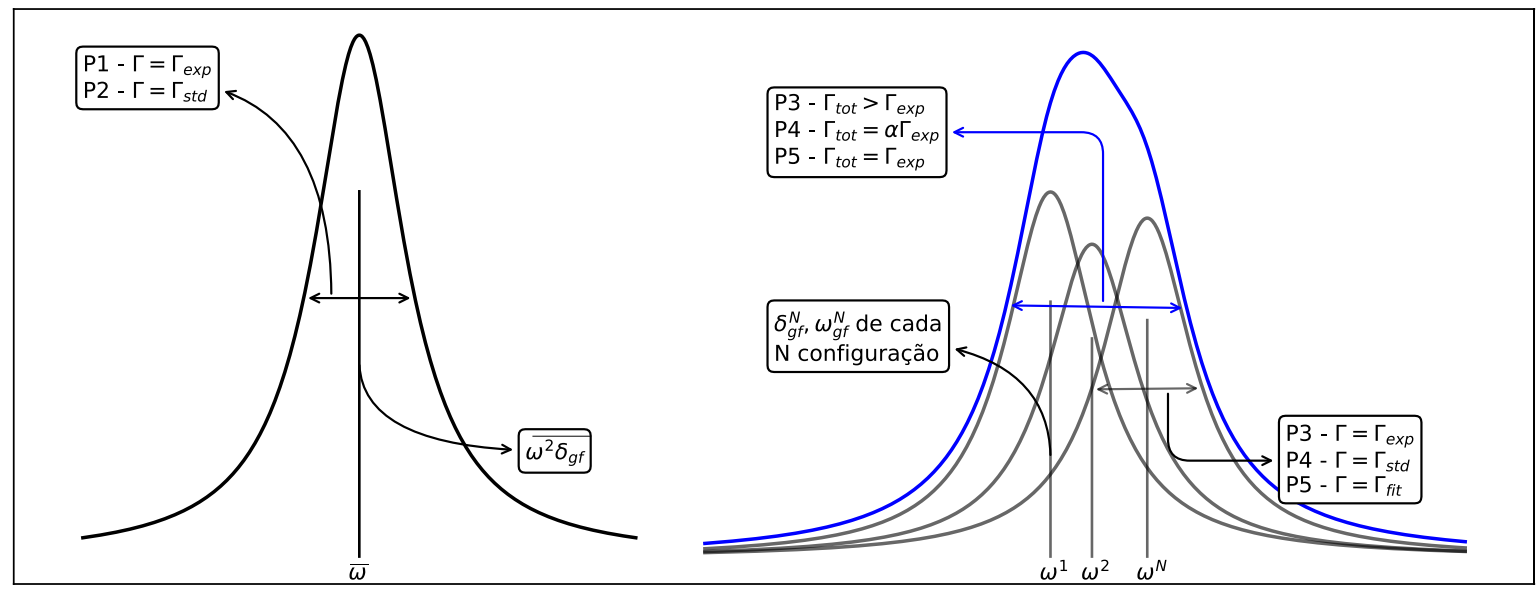

Figura 6. Ilustração dos efeitos dos diferentes procedimentos na largura de linha do espectro total.

Os efeitos de alargamento espectral envolvidos nos processos de absorção têm origem em contribuições homogêneas e não homogêneas. As contribuições homogêneas estão relacionadas com a flexibilidade do soluto enquanto as contribuições não homogêneas são dependentes da interação soluto-solvente. Dentro destas observações é evidente que diferentes formas de realizar simulações moleculares impactam nas contribuições homogêneas e não homogêneas. Por exemplo, ao considerarmos a pNA com geometria rígida durante a simulação molecular estamos negligenciando as contribuições homogêneas em P2 e P4 $\left(\Gamma=\Gamma_{s t d}\right)$. Por outro lado, a contribuição não homogênea é incluída ao menos uma vez em P3 e P4 - uma vez pelo espectro médio e outra vez por $\Gamma=\Gamma_{\text {exp }}$ já conter tal contribuição para P3 e através do $\Gamma=\Gamma_{s t d}$ para P4. O ajuste realizado para P5 é uma forma de evitar tanto a falta quanto o excesso de tais contribuições e deve resultar em melhores descrições do alargamento espectral. Para simulações com BOMD, onde a geometria da pNA é considerada flexível, os espectros obtidos tanto com P1 quanto com P2 incluem ao menos uma vez ambas as contribuições com $\Gamma=\Gamma_{\exp }$ para P1 e devido a ambos os efeitos de alargamento estarem presentes em $\Gamma=\Gamma_{s t d}$ para P2. Já os procedimentos $\mathrm{P} 3$ e P4 incluem duas vezes as duas contribuições, uma vez da mesma forma que P1 e P2 e outra devido à obtenção do espectro médio. Estas considerações estão sumarizadas na Tabela 3 . 
Tabela 3. Síntese dos diferentes procedimentos adotados para a obtenção do espectro de A2F incluindo as respectivas atribuições dos valores de $\Gamma$, das equações utilizadas para obter os espectros e das contribuições presentes em cada procedimento. ${ }^{*} G_{R}-$ Geometria rígida, $G_{F}-$ Geometria flexível.

\begin{tabular}{|c|c|c|c|c|}
\hline & $\Gamma$ & Seção de choque de $\mathrm{A} 2 \mathrm{~F}-\sigma$ & $\begin{array}{l}\text { Contribuição } \\
\text { não } \\
\text { homogênea }\end{array}$ & $\begin{array}{l}\text { Contribuição } \\
\text { homogênea* }\end{array}$ \\
\hline $\mathrm{P} 1$ & $\begin{array}{c}\Gamma_{\text {exp }} \\
\text { (do espectro } \\
\text { experimental) }\end{array}$ & \multirow{2}{*}{$\frac{4 \pi^{3}}{c^{2}} \sum_{f} \overline{\omega^{2} \delta_{0 f}} g\left(2 \omega, \overline{\omega_{0 f}} ; \Gamma\right)$} & Incluído & $\begin{array}{l}\mathrm{G}_{\mathrm{R}} \text { - Incluído } \\
\mathrm{G}_{\mathrm{F}} \text { - Incluído }\end{array}$ \\
\hline $\mathrm{P} 2$ & $\begin{array}{c}\Gamma_{s t d} \\
\text { (dos cálculos de QM) } \\
\end{array}$ & & Incluído & $\begin{array}{c}\mathrm{G}_{\mathrm{R}} \text { - Não incluído } \\
\mathrm{G}_{\mathrm{F}} \text { - Incluído }\end{array}$ \\
\hline P3 & $\begin{array}{c}\Gamma_{\exp } \\
\text { (do espectro } \\
\text { experimental) }\end{array}$ & \multirow{3}{*}{$\frac{4 \pi^{3}}{c^{2}} \frac{1}{N_{\text {conf }}} \sum_{f, N}\left(\omega^{N}\right)^{2} \delta_{0 f}^{N} g\left(2 \omega, \omega_{0 f}^{N} ; \Gamma\right)$} & $\begin{array}{c}\text { Incluído } \\
\text { duas vezes }\end{array}$ & $\begin{array}{c}\mathrm{G}_{\mathrm{R}}-\text { Incluído } \\
\mathrm{G}_{\mathrm{F}}-\text { Incluído duas vezes }\end{array}$ \\
\hline P4 & $\begin{array}{c}\Gamma_{\text {std }} \\
\text { (dos cálculos de QM) }\end{array}$ & & $\begin{array}{c}\text { Incluído } \\
\text { duas vezes }\end{array}$ & $\begin{array}{c}\mathrm{G}_{\mathrm{R}} \text { - Não incluído } \\
\mathrm{G}_{\mathrm{F}} \text { - Incluído duas vezes }\end{array}$ \\
\hline P5 & $\begin{array}{c}\Gamma_{f i t} \\
\text { (do ajuste do espectro } \\
\text { experimental) }\end{array}$ & & Incluído & Incluído (via $\left.\Gamma_{f i t}\right)$ \\
\hline
\end{tabular}

\subsubsection{Discussões}

As geometrias de mínima energia da pNA isolada e com PCM foram confirmadas pelo sinal da segunda derivada da energia. Todas as geometrias obtidas apresentam características planares com os hidrogênios ligados ao nitrogênio formando uma estrutura piramidal. A geometria média obtida da simulação BOMD em água está em boa concordância com a geometria obtida considerando a aproximação PCM.

Os momentos de dipolo do estado fundamental da pNA foram obtidos utilizando MP2 (6.2 D) e CAM-B3LYP (6.8 D), estes resultados concordam com valores teóricos prévios reportados na literatura $(5.2 \mathrm{D}-7.0$ D) $[112,121,125]$. Os resultados incluindo os efeitos de solventes utilizando as aproximações PCM e ASEC estão presentes na Tabela 4. O momento de dipolo do estado fundamental aumenta com a polaridade do solvente, sendo os valores obtidos com ASEC maiores que os obtidos com PCM. Além disso, o valor do momento de dipolo médio obtido para água-BOMD utilizando somente cargas pontuais para representar as moléculas de solvente é equivalente ao valor obtido utilizando ASEC. O momento de dipolo do estado fundamental é estimado ser entre 5.2 D e 6.5 D [118,141] em dioxano.

Pela análise das funções de distribuição radial de pares, nós observamos que a primeira camada de solvatação é composta por 16 moléculas de solvente de dioxano e DMSO, enquanto em água esse número aumenta para 33 e 39 moléculas respectivamente nas simulações com MC (água) e com BOMD (água-BOMD). Os valores médios (e os valores máximos) dos números de moléculas realizando ligações de hidrogênio são 1.9 (3), 3.3 (6) e 3.8 (6) respectivamente para as simulações da pNA em DMSO, água e água-BOMD. Embora as 
moléculas de dioxano sejam consideradas aceptoras de ligações de hidrogênio [142], em nossas análises nenhuma ligação de hidrogênio foi observada entre as moléculas de dioxano do solvente e a pNA.

Tabela 4. Momento de dipolo do estado fundamental (em Debye). * Resultados obtidos aproximando as moléculas de solvente como cargas pontuais.

\begin{tabular}{|c|c|c|c|c|}
\hline & dioxano & DMSO & água & água-BOMD \\
\hline PCM - MP2 & 7.13 & 8.54 & 8.58 & - \\
\hline PCM - CAM-B3LYP & 7.82 & 9.35 & 9.39 & - \\
\hline ASEC - CAM-B3LYP & 8.14 & 9.60 & 9.73 & $11.0 \pm 1.7^{*}$ \\
\hline
\end{tabular}

A transição $\pi \rightarrow \pi^{*}$, permitida em ambos os processos de A1F e A2F, é caracterizada pelos orbitais moleculares HOMO-LUMO ${ }^{2}$. Os orbitais moleculares obtidos são similares para os cálculos da pNA isolada e em solvente. Portanto, na Figura 7 apresentamos somente os orbitais de KS obtidos para a pNA isolada. Para a pNA isolada, obtivemos a energia de transição em 4.31 eV (o resultado experimental é de 4.25 eV [112]) e 6.0 GM para a seção de choque de A2F (utilizando $\Gamma=0.45 \mathrm{eV}$ extraído da referência [112]). Utilizando a aproximação PCM observamos um solvatocromismo de $0.74 \mathrm{eV}$ (entre a pNA isolada e em água) o qual está em razoável concordância com o deslocamento experimental de $0.99 \mathrm{eV}$. O solvatocromismo observado utilizando ASEC é de $0.43 \mathrm{eV}$, indicando que as interações não eletrostáticas contribuem substancialmente na descrição da energia de absorção.

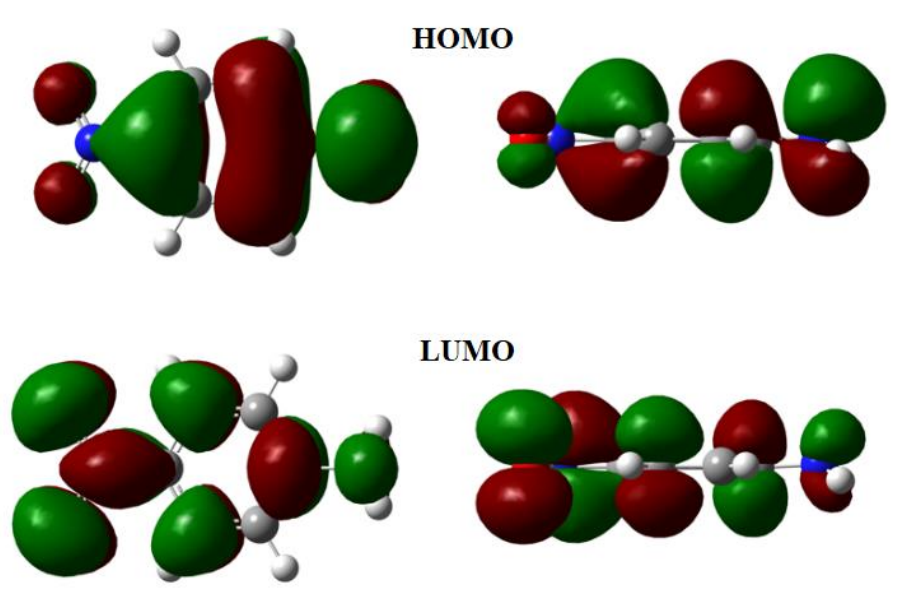

Figura 7. Orbitais de KS envolvidos na primeira transição intensa para os processos de A1F e A2F.

Os valores das amplitudes da seção de choque de A2F, obtidas utilizando a aproximação PCM e assumindo $\Gamma=\Gamma_{\text {exp }}$, foram respectivamente para os solventes dioxano, DMSO e água 
de 14.2, 38.5 e 26.0 GM. A aproximação ASEC para os efeitos eletrostáticos dos solventes se mostrou ineficaz em descrever a amplitude da seção de choque de A2F apresentando valores inferiores aos experimentais e pouco sensíveis aos diferentes solventes. Obtivemos, para a aproximação ASEC os valores 7.5, 8.6 e 5.4 GM, respectivamente, para os solventes dioxano, DMSO e água. Como referência os valores experimentais em dioxano e DMSO são, respectivamente, 8 e 12 GM. Vemos que os resultados obtidos com ASEC e com PCM não reproduzem corretamente a tendência das intensidades.

As aproximações simplificadas dos solventes (PCM e ASEC) não foram suficientes para simular a seção de choque de $\mathrm{A} 2 \mathrm{~F}$ da pNA, exigindo uma abordagem mais detalhada das interações com os solventes como a S-QM/MM. Iremos, novamente, simular a seção de choque de A2F da pNA utilizando os três solventes (dioxano, DMSO e água) considerando de forma explícita algumas moléculas de solvente nos cálculos das intensidades de A2F. As análises realizadas com os resultados obtidas utilizando simulações de MC foram identificadas como: 2- dioxano quando consideramos explicitamente as duas moléculas de dioxano mais próximas; HB-DMSO quando consideramos explicitamente as moléculas de DMSO que fazem ligações de hidrogênio com a pNA; 5-DMSO quando consideramos explicitamente as 5 moléculas de DMSO mais próximas; [HB]15-água quando consideramos explicitamente as [moléculas de água que fazem ligações de hidrogênio com a pNA] 15 moléculas de água mais próximas. Além das moléculas explícitas, as 250 moléculas de solvente mais próximas foram consideradas como cargas pontuais nos cálculos. Para as simulações utilizando BOMD consideramos as moléculas de água que fazem ligações de hidrogênio (HB-água-BOMD) e as demais como cargas pontuais. Ao total foram extraídas 100 configurações estatisticamente descorrelacionadas das simulações com MC e 200 configurações igualmente espaçadas em 125 fs das simulações com BOMD.

Para cada simulação obtivemos os valores máximos da seção de choque de A2F e a respectiva energia dos máximos analisando os espectros construídos considerando cinco procedimentos de convolução espectral. A representação gráfica e os resultados numéricos estão presentes na Figura 9 e na Tabela 5. As energias de transição $\omega_{\max }$, obtidas utilizando os P1-P5, correspondentes aos valores máximos da seção de choque de A2F mostraram variações menores que $0.03 \mathrm{eV}$ para as simulações com as geometrias rígidas e variações menores que 0.1 eV para a simulação com geometrias flexíveis. Por outro lado, as amplitudes da seção de choque de A2F calculadas variaram em mais de 2 vezes entre os diferentes procedimentos. Esta grande variação está principalmente relacionada com a forma de definir os valores de $\Gamma$, 
evidenciando a necessidade de aplicar um protocolo adequado para atribuir o valor de $\Gamma$, e que considere também as limitações devido à escolha da forma de simulação molecular. Tais considerações são mais importantes quando simulações com geometrias rígidas são realizadas, uma vez que o conjunto de configurações extraídos das simulações reproduzem os efeitos das contribuições não homogêneas no alargamento espectral e negligenciam as contribuições homogêneas.

Devido ao uso de um conjunto de configurações, provenientes de simulações com moléculas rígidas, reproduzir somente as contribuições não homogêneas para o alargamento espectral, é fisicamente justificável a utilização do parâmetro de ajuste $\Gamma_{\text {fit }}$ como um complemento para reproduzir o alargamento espectral observado no experimento. Esse parâmetro de ajuste representa de certo modo a contribuição homogênea. Esta é a ideia principal do procedimento de convolução P5, o qual forneceu neste estudo as melhores descrições da seção de choque de A2F. O estado excitado de menor energia da pNA é do tipo $n \rightarrow \pi^{*}$ e dependendo das interações soluto-solvente ele inverte de ordem com o estado intenso $\pi \rightarrow \pi^{*}$ fornecendo dados espúrios para a estimativa de $\Gamma$ considerando o desvio padrão da energia de transição $\left(\Gamma_{s t d}\right)$. Para evitar este efeito seria necessário classificar as transições de todas as configurações para obter uma estimativa mais adequada das médias $\overline{\omega^{2} \delta_{g f}}$ e $\overline{\omega_{g f}}$ e de $\Gamma_{s t d}$ quando aplicável. Embora uma análise visual dos orbitais envolvidos nas transições frequentemente podem ajudar a caracterizar a transição eletrônica, em muitas vezes não é possível identificar com clareza se a transição é do tipo $n \rightarrow \pi^{*}$ ou $\pi \rightarrow \pi^{*}$. Essa é a razão para a largura de linha total obtida com P1 ser maior que o valor experimental. Uma comparação direta mostra que o valor da largura total segue o comportamento $\mathrm{P} 2<\mathrm{P} 4<\mathrm{P} 1<\mathrm{P} 3$, essa informação pode ser observada na Figura 8. 


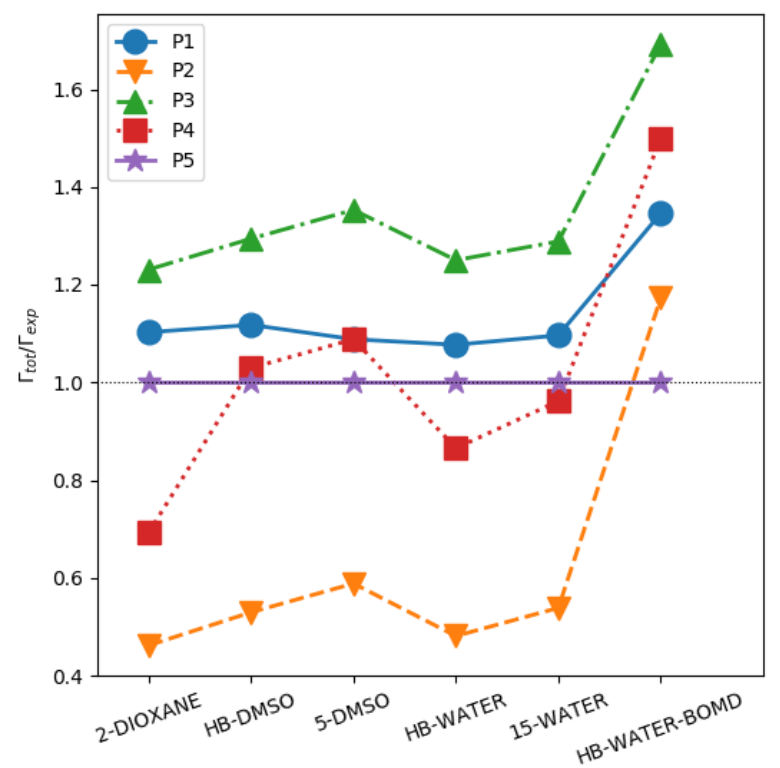

Figura 8. Comparação da largura de linha total obtida para o espectro de A2F com o valor experimental relacionado com o espectro de AlF.

$\mathrm{O}$ aumento da intensidade da seção de choque de A2F entre os solventes dioxano e DMSO foi de $18 \%$ e $10 \%$ respectivamente para P1 e P3, por outro lado, P2 e P4 mostraram tendência contrária com uma redução de 15\%. Os máximos obtidos com P5 resultaram num aumento de $27 \%$ entre os mesmos solventes e representam nossa melhor descrição teórica quando comparada com o aumento de $50 \%$ obtidos dos dados experimentais. Aumentando o número de moléculas explícitas de solvente obtivemos uma pequena variação em direção a valores numericamente mais próximos dos experimentais embora estas pequenas melhorias não justifiquem a demanda computacional adicional. Outro ponto interessante observado para P5 é a boa concordância entre os valores obtidos em água utilizando a "barata" simulação com MC e a "custosa" simulação com BOMD. Adicionalmente obtivemos os espectros de A2F utilizando o funcional B3LYP. Os valores de $\Gamma_{f i t}$ obtidos com este funcional estão em boa concordância com os valores obtidos com o funcional CAM-B3LYP, resultando em diferenças menores que $0.03 \mathrm{eV}$. Por outro lado, os resultados dos máximos da seção de choque de A2F obtidos com o funcional B3LYP são 30\% inferiores. Esta similaridade dos resultados de $\Gamma_{f i t}$ indica, de certo modo, uma boa estimativa das contribuições homogêneas no alargamento espectral. 


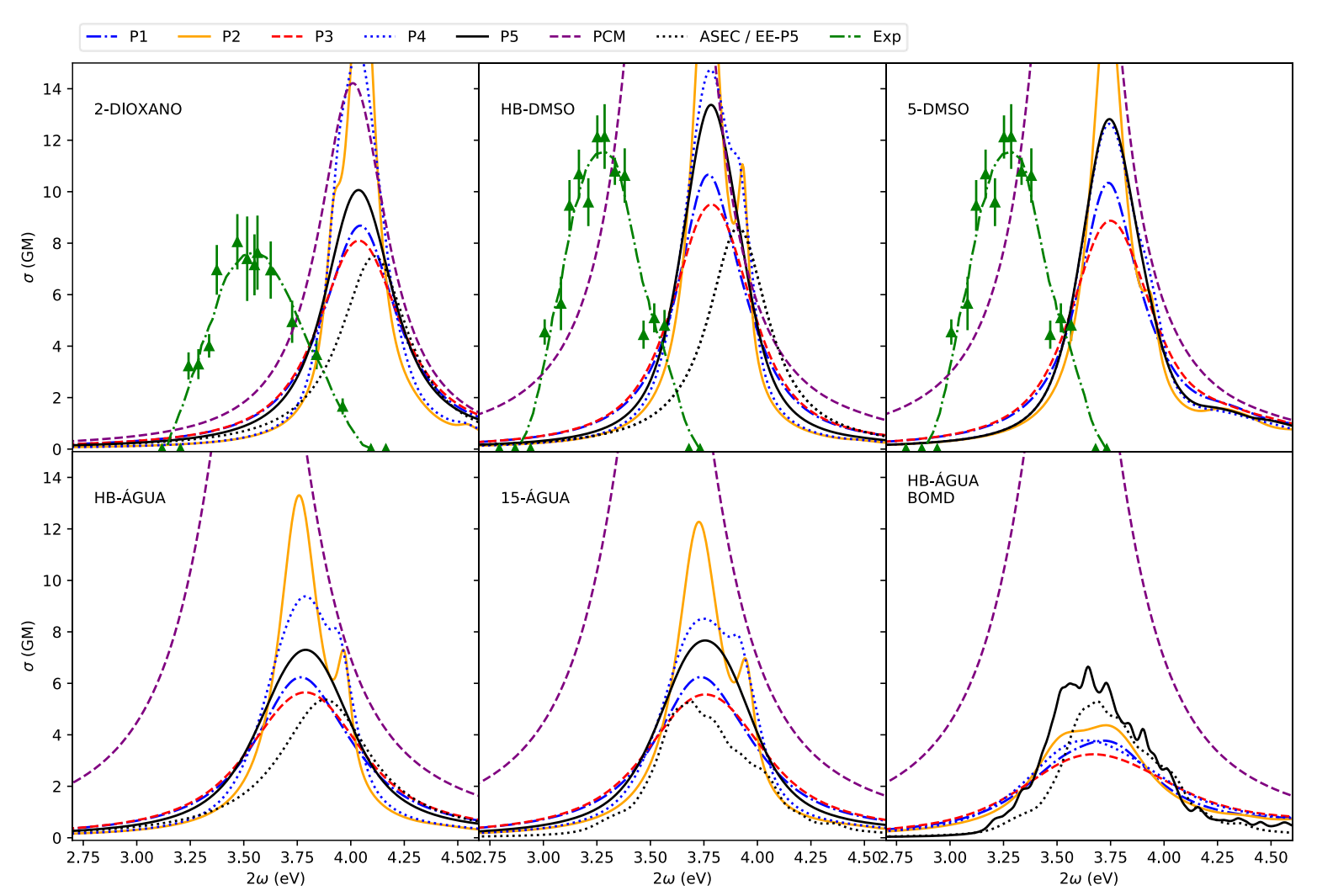

Figura 9. Espectros de A2F obtidos considerando os cinco procedimentos adotados e utilizando as aproximações PCM e ASEC para o solvente. Os dados experimentais foram extraídos da referência [127].

Como mencionado, nós assumimos que $\Gamma_{f i t}$ descreve, ao menos de forma semi quantitativa, a contribuição homogênea do alargamento espectral. Assim, é esperado que o valor de $\Gamma_{f i t}$ seja maior para as simulações realizadas com MC uma vez que nenhuma contribuição homogênea é estimada com geometria rígida. Este comportamento é observado comparando os valores obtidos para $\Gamma_{\text {fit }}$ de $0.37 \mathrm{eV}$ e $0.06 \mathrm{eV}$, respectivamente, para as simulações HB-água e HB-água-BOMD. Duas análises complementares foram realizadas para compreender melhor as contribuições dos alargamentos espectrais. Na primeira análise, identificada como água-isolada, obtivemos os valores das energias de transição e das intensidades de A2F para as configurações HB-água-BOMD considerando somente a pNA (sem a inclusão de moléculas de água), ou seja, a informação referente à solvatação está restrita a efeitos vibracionais. Na segunda análise uma nova simulação BOMD foi realizada para a pNA isolada e a identificamos com o termo "isolada". Comparando o resultado destas duas simulações é possível mensurar os efeitos da solvatação na contribuição homogênea do alargamento espectral. Para essa comparação utilizamos como referência os valores de $\Gamma_{\text {tot }}$ obtidos com P2 (que inclui tanto a contribuição homogênea e não homogênea). Os efeitos de solvente na flexibilidade da pNA reduziram a intensidade da seção de choque de A2F em 4.4 GM entre as simulações isolada e água-isolada indicando grande dependência dos efeitos de 
solvente. Já os resultados para $\Gamma_{\text {tot }}$ obtidos entre estas duas simulações mostram valores da ordem de $0.3 \mathrm{eV}$ que são compatíveis com o valor $\Gamma_{f i t}=0.37$ e $0.33 \mathrm{eV}$ obtidos respectivamente para HB-água e 15-água. Os valores estão apresentados na Tabela 6.

Tabela 5. Seção de choque de A2F da pNA em solução. Os valores das energias de transição referentes a intensidade máxima de absorção $\left(\omega_{\max }\right)$ e das larguras de linha $\Gamma$ estão em eV e os valores das seções de choque de A2F $(\sigma)$ estão em GM. Os resultados experimentais (EXP) foram extraídos das referências [112,127]. Os valores ajustados de $\Gamma_{\text {fit }}$ utilizados em $P 5$ estão apresentados entre parênteses.

\begin{tabular}{|c|c|c|c|c|c|c|c|c|c|}
\hline \multirow{4}{*}{$\begin{array}{c}\text { PCM }^{1} \\
\text { ASEC }^{1,2}\end{array}$} & \multicolumn{3}{|c|}{ 2-dioxano } & \multicolumn{3}{|c|}{ HB-DMSO } & \multicolumn{3}{|c|}{ 5-DMSO } \\
\hline & $\omega_{\max }$ & $\Gamma_{t o t}$ & $\sigma$ & $\omega_{\max }$ & $\Gamma_{t o t}$ & $\sigma$ & $\omega_{\max }$ & $\Gamma_{t o t}$ & $\sigma$ \\
\hline & 4.01 & 0.39 & 14.2 & 3.59 & 0.34 & 38.5 & - & - & - \\
\hline & 4.11 & 0.39 & 7.5 & 3.91 & 0.34 & 8.6 & - & - & - \\
\hline $\mathrm{P} 1$ & 4.04 & 0.43 & 8.7 & 3.77 & 0.38 & 10.7 & 3.74 & 0.37 & 10.3 \\
\hline $\mathrm{P} 2$ & 4.05 & 0.18 & 20.9 & 3.77 & 0.18 & 20.6 & 3.74 & 0.20 & 17.8 \\
\hline P3 & 4.04 & 0.48 & 8.1 & 3.79 & 0.44 & 9.5 & 3.75 & 0.46 & 8.9 \\
\hline P4 & 4.03 & 0.27 & 15.5 & 3.78 & 0.35 & 14.7 & 3.75 & 0.37 & 12.6 \\
\hline P5 & 4.04 & $\begin{array}{c}0.39 \\
(0.30)\end{array}$ & 10.1 & 3.79 & $\begin{array}{c}0.34 \\
(0.21)\end{array}$ & 13.4 & 3.75 & $\begin{array}{c}0.34 \\
(0.20)\end{array}$ & 12.8 \\
\hline EXP & 3.51 & 0.39 & 8 & 3.18 & 0.34 & 12 & 3.18 & 0.34 & 12 \\
\hline & & AB-águ & & & 15-água & & $\mathrm{HB}$ & gua-B & \\
\hline & $\omega_{\max }$ & $\Gamma_{t o t}$ & $\sigma$ & $\omega_{\max }$ & $\Gamma_{t o t}$ & $\sigma$ & $\omega_{\max }$ & $\Gamma_{t o t}$ & $\sigma$ \\
\hline $\mathrm{PCM}^{1}$ & 3.57 & 0.52 & 26.0 & - & - & - & - & - & - \\
\hline $\mathrm{ASEC}^{1,2}$ & 3.88 & 0.52 & 5.4 & - & - & - & 3.69 & 0.52 & 5.3 \\
\hline P1 & 3.77 & 0.56 & 6.2 & 3.74 & 0.57 & 6.2 & 3.72 & 0.70 & 3.8 \\
\hline P2 & 3.76 & 0.25 & 13.3 & 3.73 & 0.28 & 12.3 & 3.73 & 0.61 & 4.4 \\
\hline P3 & 3.79 & 0.65 & 5.7 & 3.75 & 0.67 & 5.6 & 3.67 & 0.88 & 3.2 \\
\hline P4 & 3.79 & 0.45 & 9.4 & 3.75 & 0.50 & 8.51 & 3.63 & 0.78 & 3.8 \\
\hline P5 & 3.79 & $\begin{array}{c}0.52 \\
(0.37)\end{array}$ & 7.3 & 3.75 & $\begin{array}{c}0.52 \\
(0.33)\end{array}$ & 7.7 & 3.65 & $\begin{array}{c}0.52 \\
(0.06)\end{array}$ & 6.7 \\
\hline EXP & 3.26 & 0.52 & - & 3.26 & 0.52 & - & 3.26 & 0.52 & - \\
\hline
\end{tabular}

${ }^{1}$ Os resultados obtidos na aproximação PCM e ASEC foram apresentados somente uma vez para cada solvente.

${ }^{2}$ Os resultados de HB-água-BOMD foram obtidos considerando todas as moléculas do solvente como cargas pontuais obtidos com P5.

Tabela 6. Energia de transição relacionada ao máximo valor da seção de choque de $A 2 F\left(\omega_{\max }\right)$ em eV, largura de linha total $\Gamma_{\text {tot }}$ em eV e seção de choque de A2F $(\sigma)$ em GM obtidos de simulações BOMD.

\begin{tabular}{|c|c|c|c|}
\hline & $\omega_{\max }$ & $\Gamma_{\text {tot }}$ & $\sigma$ \\
\hline isolada & 4.16 & 0.28 & 10.3 \\
\hline água-isolada & 4.01 & 0.33 & 6.9 \\
\hline HB-água-BOMD & 3.73 & 0.61 & 4.4 \\
\hline
\end{tabular}

\subsubsection{Conclusões}

A seção de choque de A2F é uma propriedade importante para aplicações baseadas em processos de A2F. Uma vez que a seção de choque de A2F depende do alargamento espectral, o valor da largura de linha $\Gamma$ não deve ser arbitrariamente escolhido. Neste trabalho diferentes formas de atribuir o valor de $\Gamma$ combinadas com diferentes formas de construir o espectro foram avaliados para simular a seção de choque de A2F da para-Nitroanilina em meio líquido. A diferença entre os valores obtidos para as intensidades de A2F estão relacionadas com 
subestimativa ou superestimativa dos efeitos de alargamento provenientes dos diferentes procedimentos de convolução e de atribuição de valores para $\Gamma$.

O novo procedimento proposto para atribuir o valor de $\Gamma$ impõe que a largura do espectro final de $\mathrm{A} 2 \mathrm{~F}$ seja o mesmo que o obtido no espectro experimental de $\mathrm{A} 1 \mathrm{~F}$ evitando assim possíveis desbalanços dos efeitos de alargamento. Esta imposição está fundamentada na condição do estado excitado de interesse ser acessível tanto pelo processo de A1F quanto pelo processo de $\mathrm{A} 2 \mathrm{~F}$ e não deve ser adequado caso esta condição não seja satisfeita. O procedimento de ajuste consiste em encontrar um valor de $\Gamma$ para centenas de configurações oriundas de simulações moleculares de forma que o espectro médio reproduza $\Gamma_{t o t}=\Gamma_{\text {exp }}$. Este procedimento forneceu os resultados mais precisos para as amplitudes da seção de choque de A2F da pNA em solução.

Para casos onde não existem dados experimentais disponíveis, nossos resultados mostraram que um protocolo combinando simulações com geometrias rígidas e flexíveis pode ser utilizado para estimar o alargamento espectral e assim construir o espetro de A2F. Neste protocolo, a contribuição homogênea do valor de $\Gamma$ de uma transição eletrônica é obtida como o desvio padrão da energia de transição. Essas energias de transição foram computadas para configurações da pNA isolada. Contudo, as configurações foram geradas com BOMD considerando moléculas explícitas de solvente. Construímos o espectro de A2F incluindo a contribuição não homogênea ao convoluir as intensidades de A2F obtidas de configurações extraídas de simulações com geometrias rígidas.

Finalmente, nossos resultados indicam que o uso do procedimento de ajuste para estimar o valor de $\Gamma$ é uma ótima escolha quando a metodologia S-QM/MM é adotada para incluir os efeitos de solventes no estudo do processo da A2F. Neste trabalho apresentamos algumas alternativas para obter o espectro de $\mathrm{A} 2 \mathrm{~F}$ de forma menos arbitrária quanto à definição do valor de $\Gamma$. Acreditamos que estes resultados serão úteis em futuros trabalhos principalmente no que se refere ao desenvolvimento de novos compostos para aplicações que se dão em meio líquido. 


\subsection{Efeitos estruturais e de solvente na descrição da absorção de dois fótons de piridínio N-fenolato}

\subsubsection{Introdução}

Perturbações externas provenientes de interações com o ambiente externo influenciam a geometria e a estrutura eletrônica molecular. Essas perturbações, em nível microscópico, impactam na mobilidade eletrônica resultando em variações na quantidade de carga transferida e no respectivo deslocamento dessa carga durante excitações eletrônicas. As perturbações devido aos efeitos de solventes podem ser incluídas utilizando diversos protocolos, considerando tanto aproximações implícitas para o solvente quanto inclusão de moléculas explícitas de solventes. Por outro lado, utilizar campos elétricos estáticos para aproximar os efeitos de interação eletrostática entre o soluto-solvente é uma alternativa simples e utilizada principalmente para induzir variações entre espécies neutras e zwiterionicas [143-145]. Estudos sobre as polarizabilidades $\boldsymbol{\alpha}, \boldsymbol{\beta}$ e $\boldsymbol{\gamma}$ [146], sobre a A2F mediadas por fônons [147], sobre a modulação da absorção de dois fótons e sobre os efeitos de limitação óptica no fotodiodo HgCdTe [148,149] são alguns exemplos de estudos encontrados na literatura utilizando campos eletrostáticos.

Sabendo que um aumento da transferência de carga durante excitações eletrônicas favorece processos ONLs, portanto, compreender a relação entre esses fenômenos deve facilitar o desenvolvimento de novos compostos com maiores potenciais tecnológicos. Indícios de transferência de carga podem ser obtidos pelo solvatocromismo. Estimativas teóricas da transferência de carga durante excitações eletrônicas podem ser obtidas analisando: (i) o momento de dipolo permanente e o momento de dipolo de transição entres os estados de interesse; e/ou (ii) a densidade eletrônica dos estados inicial e final [108,109].

Os compostos de piridínio N-fenolato Betaína (Figura 10), referenciados nesse texto apenas por para-betaína (pBT) e ortho-betaína (oBT), são potenciais candidatos a aplicações no campo da ONL $[150,151]$. Contudo, as estruturas moleculares desses sistemas dependem do ambiente externo onde se encontram. A diferença dos valores do ângulo torcional entre os anéis é maior para a $\mathrm{oBT}$, indo de $30^{\circ}$ para a oBT isolada para $60^{\circ}$ para a oBT em água $[152,153]$. A variação da probabilidade de transição via dois fótons $(\delta)$ devido a mudanças do ângulo interno de rotação entre os anéis foi previamente estimada utilizando o método semi-empírico GRINDOL para a pBT isolada e em meio aquoso (utilizando método baseado em dipolos de Langevin e Monte Carlo para considerar as interações soluto-solvente) [154]. Utilizando o 
modelo de dois níveis (two-level model - 2LM) e o método soma-sobre-estados considerando 600 estados intermediários, os autores reportaram valores de $\delta$ muito maiores para a pBT isolada do que em água.

Nesse estudo estamos estendendo a análise da A2F em função da torção entre os anéis para a oBT isolada e avaliamos os efeitos de perturbações externas como a aplicação de campos elétricos estáticos. Também consideramos, somente para a pBT, a inclusão das interações soluto-solvente com PCM e realizamos uma polarização iterativa utilizando simulações moleculares e ASEC. Além disso, analisamos as quantidades de carga transferida durante a excitação eletrônica.

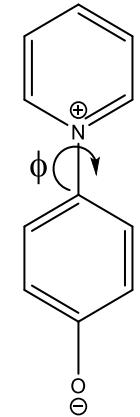

(a)

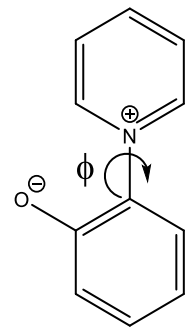

(b)

Figura 10: Estrutura química da (a) para-betaína e (b) ortho-betaína.

\subsubsection{Detalhes computacionais}

Obtivemos as geometrias utilizando o método DFT com o funcional B3LYP e com o conjunto de funções base 6-311++G(d,p). As condições de mínimo na superfície de energia potencial foram confirmadas pelo sinal da segunda derivada da energia, os quais são todos positivos. A influência da estrutura molecular nos processos de A1F e A2F foi avaliada considerando variações induzidas do ângulo torcional $\phi$ entre os anéis. As geometrias com os ângulos torcionais fixos foram re-otimizadas permitindo que os demais graus de liberdade se ajustassem para uma condição de mínimo local. Perturbações com campos estáticos (F) externos foram consideradas para avaliar as propriedades eletrônicas envolvidas no processo de A2F em três condições: (i) geometria molecular obtida na presença de $F\left(G_{F}\right)$ e propriedades eletrônicas obtidas para o composto isolado $(\mathrm{nF})$, identificada como $\mathrm{G}_{\mathrm{F}} / \mathrm{nF}$; (ii) geometria molecular obtida para o composto isolado $\left(\mathrm{G}_{\mathrm{R}}\right)$ e propriedades eletrônicas obtidas na presença de $\mathrm{F}(\mathrm{wF})$, identificada como $\mathrm{G}_{\mathrm{R}} / \mathrm{wF}$; e (iii) geometria molecular e propriedades eletrônicas obtidas na presença de $\mathrm{F}$, identificada como $\mathrm{G}_{\mathrm{F}} / \mathrm{wF}$. Variamos a intensidade de $\mathrm{F}$ em incrementos de $5 \times 10^{-4}$ u.a., até o valor máximo de $120 \times 10^{-4}$ e $150 \times 10^{-4}$ u.a. para a oBT e pBT, 
respectivamente. $\mathrm{O}$ campo $\mathrm{F}$ foi aplicado na mesma direção do momento de dipolo do estado fundamental.

Para o composto pBT obtivemos as geometrias de mínima energia com PCM para solventes de diferentes polaridades. Os solventes água, metanol, etanol, 1-propanol, 2-propanol, acetonitrila e dimetilsulfóxido foram escolhidos devido a existência de resultados experimentais para a energia de transição. Além destes solventes, também consideramos os solventes acetona, diclorometano, clorofórmio, benzeno e tolueno para contemplar solventes com menores polaridades, mesmo sabendo que a pBT não é solúvel nesses solventes.

Simulações moleculares com geometrias rígidas utilizando o método $\mathrm{MC}$ foram realizadas no programa DICE [97] de forma iterativa a fim de obter a polarização eletrônica [155] da pBT em solução aquosa. Os valores dos parâmetros de Lennard-Jones utilizados nas simulações foram definidos pelo campo de força OPLS-AA para a pBT e o campo de força SPC/E [133] para as moléculas de água. Cada simulação foi realizada para 1 molécula de soluto e 1000 moléculas de água. Consideramos $6 \times 10^{7}$ passos de MC no processo de termalização e $3 \times 10^{8}$ passos de MC no processo de produção de configurações para a metodologia S-QM/MM da polarização. O conjunto de cargas utilizado na primeira simulação foi obtido utilizando análise populacional CHELPG com PCM. Note que os valores de cargas atômicas obtidas com PCM não consideram uma amostragem preferencial das moléculas de solvente devido à efeitos termodinâmicos e de ligações específicas. Portanto, nas simulações seguintes o conjunto de cargas foi redefinido utilizando novamente o método CHELPG, mas considerando o solvente descrito pela aproximação ASEC. Mantivemos a geometria molecular, obtida em PCM, em todas as simulações. Ao final de cada simulação selecionamos as 400 moléculas de água mais próximas do soluto de 100 configurações estatisticamente descorrelacionadas para compor o ASEC. No total incluímos $4 \times 10^{4}$ cargas pontuais para representar a distribuição eletrostática média do solvente e obter e conjunto de cargas polarizadas do soluto para a próxima simulação.

Os valores da seção de choque de A2F para os dois compostos foram obtidos em função do ângulo torcional entre os anéis, em função da intensidade de campo elétrico externo e em função dos solventes aproximados com PCM. Os cálculos utilizando a teoria de resposta quadrática (QR) foram realizados no programa Dalton 2016.2 [139,140]. Para obter as propriedades eletrônicas relacionadas com a seção de choque de A2F utilizando 2LM utilizamos o programa Gaussian 09 [81]. Nesta etapa, os cálculos foram realizados utilizando o nível de cálculo CAM-B3LYP/6-311++G(d,p) e adotando o valor de $\Gamma=0.1 \mathrm{eV}$ para a largura de linha completa a meia altura. 


\subsubsection{Discussões}

Consideramos em nossas análises a transição eletrônica para o primeiro estado excitado das betaínas, o qual é do tipo $\pi \rightarrow \pi^{*}$ e envolve os orbitais moleculares HOMO-LUMO. Monitoramos essa transição enquanto alteramos $\phi$ entre $0^{\circ}$ e $180^{\circ}$ em incrementos de $5^{\circ}$, onde em cada valor de $\phi$ permitimos a relaxação da geometria. Como esperado, devido a simetria molecular, observamos um espelhamento das propriedades eletrônicas em $90^{\circ}$.

Os resultados obtidos mostram que a seção de choque de A2F $(\sigma)$ obtida com 2LM reproduz quantitativamente o valor obtido utilizando $\mathrm{QR}$, indicando a predominância de dois estados envolvidos na $\mathrm{A} 2 \mathrm{~F}$. $\mathrm{O}$ valor da seção de choque de $\mathrm{A} 2 \mathrm{~F}$ da $\mathrm{pBT}$, obtido com $\mathrm{QR}$, aumenta de 10 a 43 GM com o aumento do ângulo $\phi$ e apresenta um pequeno decréscimo quando $\phi=90^{\circ}$. Embora os resultados de Zalèsny e coautores [154] mostrem que o valor de $\sigma$ deveria ser nulo neste ponto, a instabilidade estrutural ocasionou uma quebra de simetria devido à flexibilidade dos demais graus de liberdade - proporcionando o valor de $\sigma=40 \mathrm{GM}$. Por outro lado, os valores obtidos com 2LM para a oBT chegaram a ser entre 20 e $30 \%$ maiores que o obtido com $\mathrm{QR}$, indicando que outros estados devem participar reduzindo a intensidade do processo de $\mathrm{A} 2 \mathrm{~F}$. Ainda para a oBT, os resultados obtidos para $\sigma$ mostram relativa estabilidade quando $0<\phi<60$ e observamos um decréscimo de $\sigma$ entre $60<\phi<90$ até inibição total do processo de A2F em $\phi=90$, embora os valores obtidos com 2LM sejam superiores aos obtidos com QR. Assim, podemos utilizar o 2LM para compreender esse decréscimo nessa região. Os resultados estão apresentados na Figura 11.
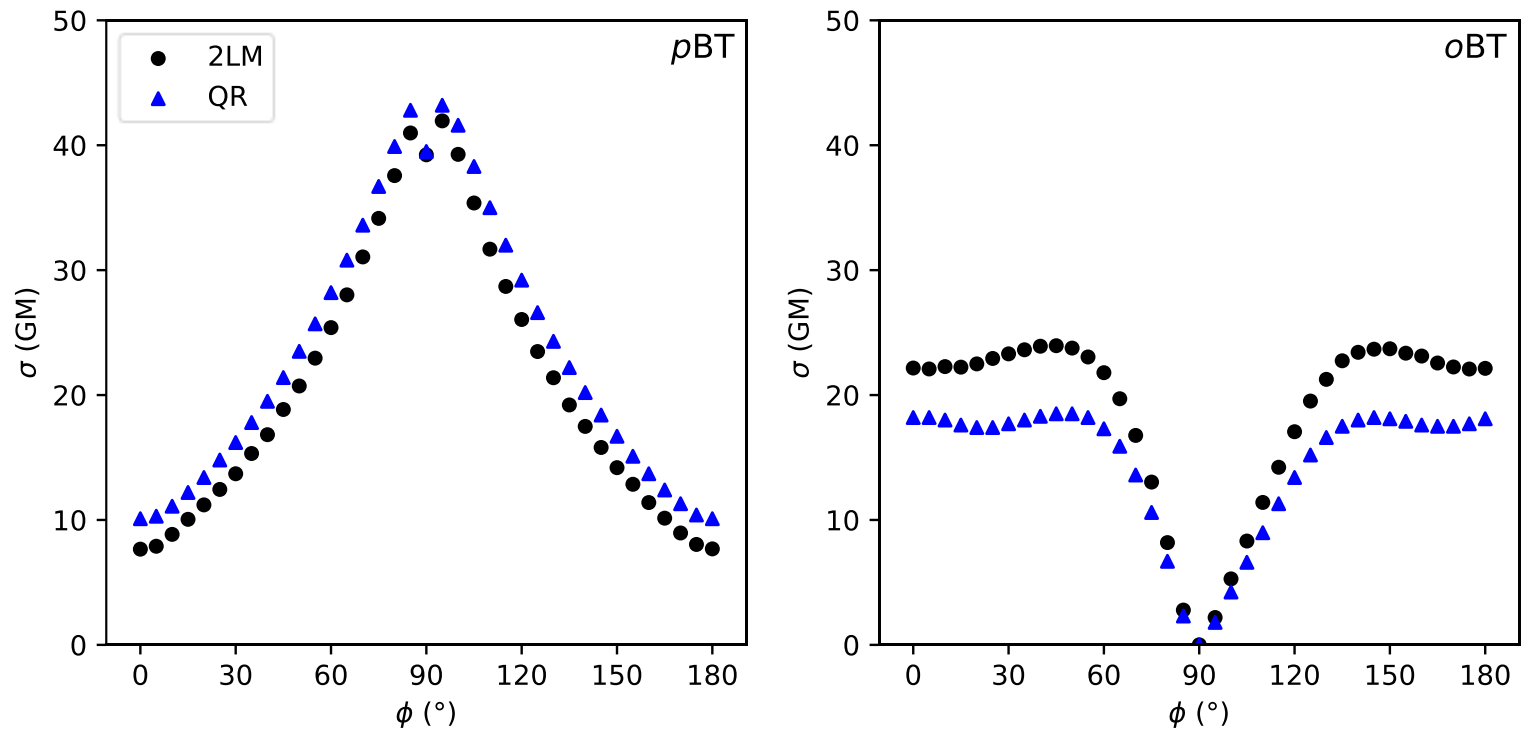

Figura 11. Seção de choque de absorção de dois fótons em função do ângulo torcional $\phi$ entre os anéis para a para-betaína (esquerda) e ortho-betaína (direita). 

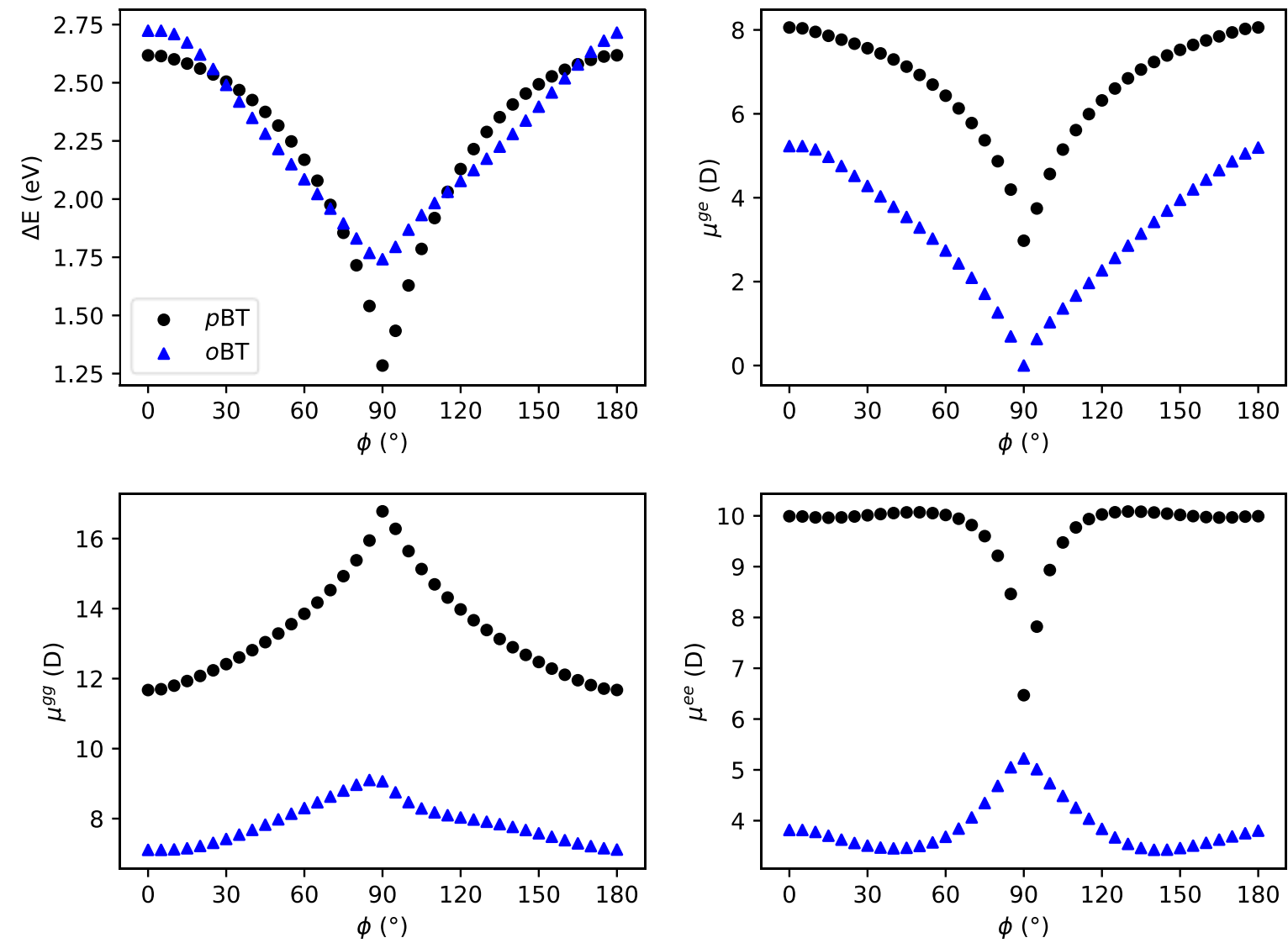

Figura 12. Propriedades eletrônicas diretamente relacionadas com o modelo de dois níveis para a seção de choque de A2F em função do ângulo $\phi$ entre os anéis. Energia de transição $\Delta E$, dipolo de transição $\mu^{g e}$, dipolo permanente do estado fundamental $\mu^{g g}$ e dipolo permanente do estado excitado $\mu^{e e}$.

A aproximação 2LM da seção de choque de A2F está relacionada com o momento de dipolo de transição $\mu^{g e}$ e com os momentos de dipolo permanente do estado fundamental $\mu^{g g}$ e do estado excitado $\mu^{e e}$ da molécula (ver equação (46) na página 39), adicionalmente analisamos as energias de transição $\Delta E$. Os valores obtidos para estas propriedades estão apresentados na Figura 12. Observamos que as propriedades eletrônicas da pBT são mais sensíveis às variações torcionais que as da oBT. O valor da energia $\Delta E$ diminuiu num regime quase linear de 2.72 a $1.74 \mathrm{eV}$ para a oBT enquanto o valor de $\Delta E$ obtido para a pBT varia de 2.62 a $1.28 \mathrm{eV}$ apresentando um decréscimo mais acentuado a partir de $\phi=60^{\circ}$. O mesmo comportamento descendente é observado para $\mu^{g e}$, porém os valores do $\mu^{g e}$ obtidos para a oBT são da ordem de $3 \mathrm{D}$ inferiores aos obtidos para a pBT chegando ao valor zero em $\phi=$ $90^{\circ}$. A pBT apresenta comportamento inverso entre os momentos de dipolo permanente: os valores de $\mu^{g g}$ aumentam continuamente até $\phi=90^{\circ}$ e os valores de $\mu^{e e}$ se mantem aproximadamente constantes até $\phi=60^{\circ}$ quando, então, diminuem abruptamente. Esse comportamento oposto induz uma variação de $8.6 \mathrm{D}$ na diferença $\mu^{e e}-\mu^{g g}$ corroborando com o aumento observado de $\sigma$. Por outro lado, os dipolos permanentes da oBT apresentam o mesmo 
comportamento em função de $\phi$, além disso, a variação $\mu^{e e}-\mu^{g g}$ é pequena. Portanto podemos afirmar, dentro da aproximação de dois níveis, que $\mu^{g e}$ é o principal modulador da intensidade da A2F para a oBT.

No estudo realizado estimamos também a quantidade de carga transferida $\mathrm{q}_{\mathrm{t}}$ durante a excitação e a distância $\mathrm{R}$ entre os pontos de acréscimo e decréscimo dessa carga. $\mathrm{O}$ excesso e a escassez de cargas devem ocorrer, respectivamente, na região dos átomos de nitrogênio e oxigênio, assim, esperamos que $\mathrm{R}$ alcance valores maiores para a pBT do que para a oBT. Por outro lado, a proximidade desses grupos de carga na molécula oBT deve favorecer a mobilidade eletrônica aumentando a quantidade de carga transferida $\mathrm{q}_{\mathrm{t}}$. De fato, esses comportamentos foram confirmados pela análise das densidades eletrônicas dos estados fundamental e excitado e podem ser observados na Figura 13. O aumento de $\mathrm{q}_{\mathrm{t}}$ e de $\mathrm{R}$ em função de $\phi$ também pode ser compreendido analisando os orbitais moleculares HOMO e LUMO da pBT (Figura 14). A torção faz com que o orbital molecular HOMO fique localizado mais próximo ao oxigênio, concentrando a densidade eletrônica, enquanto aumenta a delocalização do orbital LUMO sobre a molécula, criando assim uma densidade eletrônica no outro extremo da pBT.
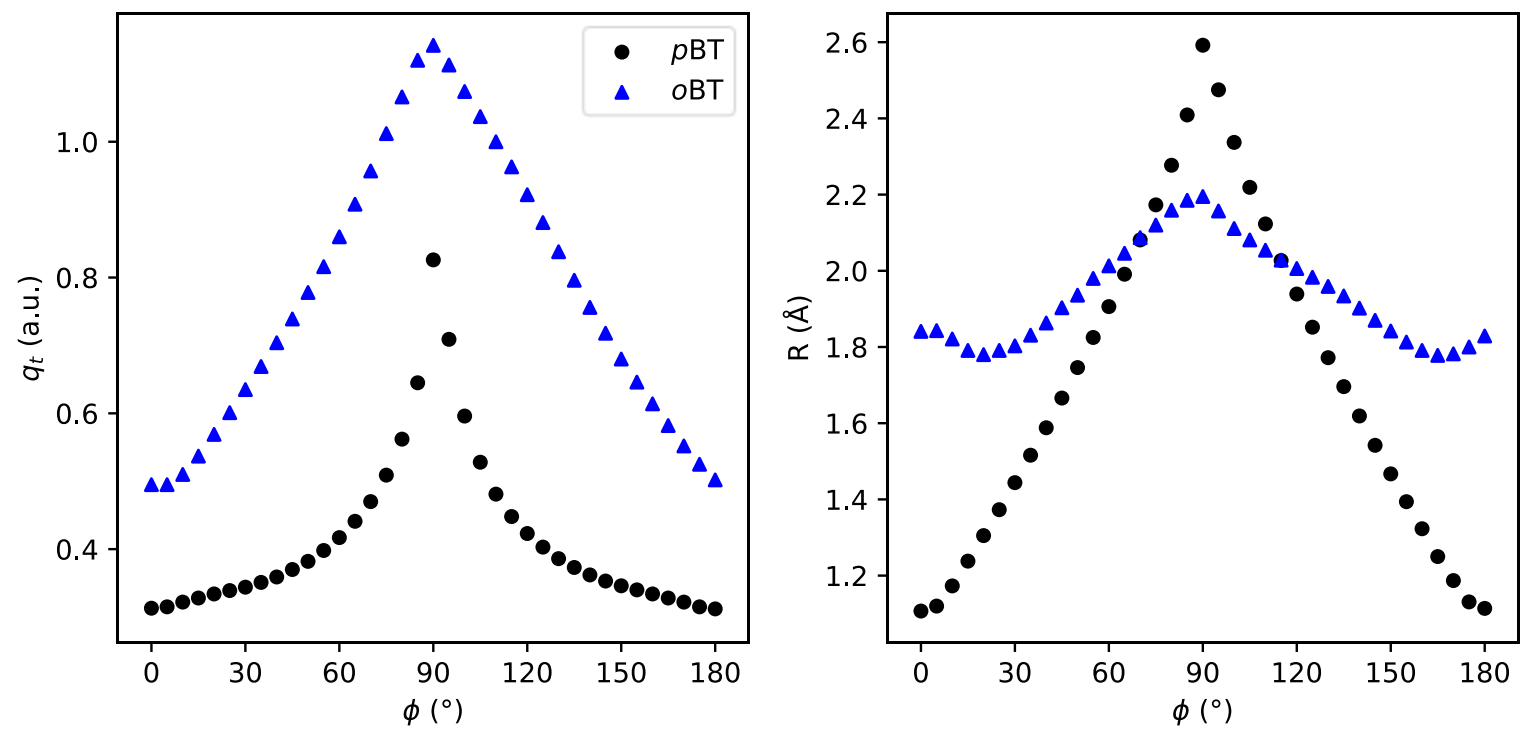

Figura 13. Quantidade de carga $q_{t}$ transferida durante a excitação e distância de deslocamento $R$ entre os pontos de decréscimo e acréscimo da carga em função do ângulo $\phi$ entre os anéis.

Notamos uma relação linear entre o aumento da carga transferida durante a excitação e da seção de choque de A2F para a pBT entre os valores de $0.3<\mathrm{q}_{\mathrm{t}}<0.5$. Por outro lado, os resultados de $\sigma\left(q_{t}\right)$ obtidos para a oBT mostram valores aproximadamente constante entre 0.5 $<\mathrm{q}_{\mathrm{t}}<0.8$ quando ocorre um decréscimo até $\sigma(1.14)=0$. As variações de $\mathrm{q}_{\mathrm{t}}$ foram induzidas 
devido aos diferentes valores de $\phi$, e, portanto, são uma consequência da estrutura geométrica do composto. Os resultados estão sumarizados na Figura 15.

HOMO
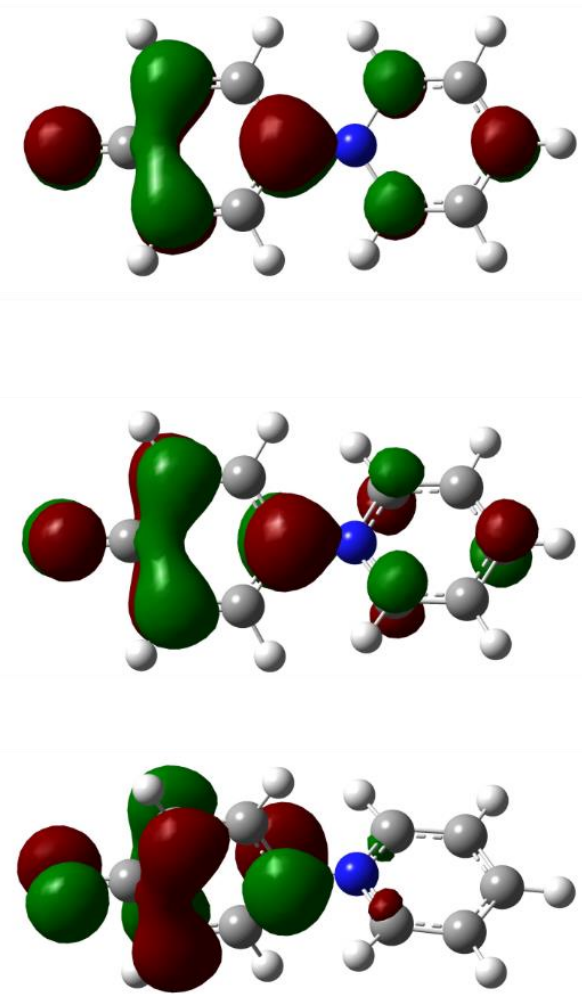

LUMO
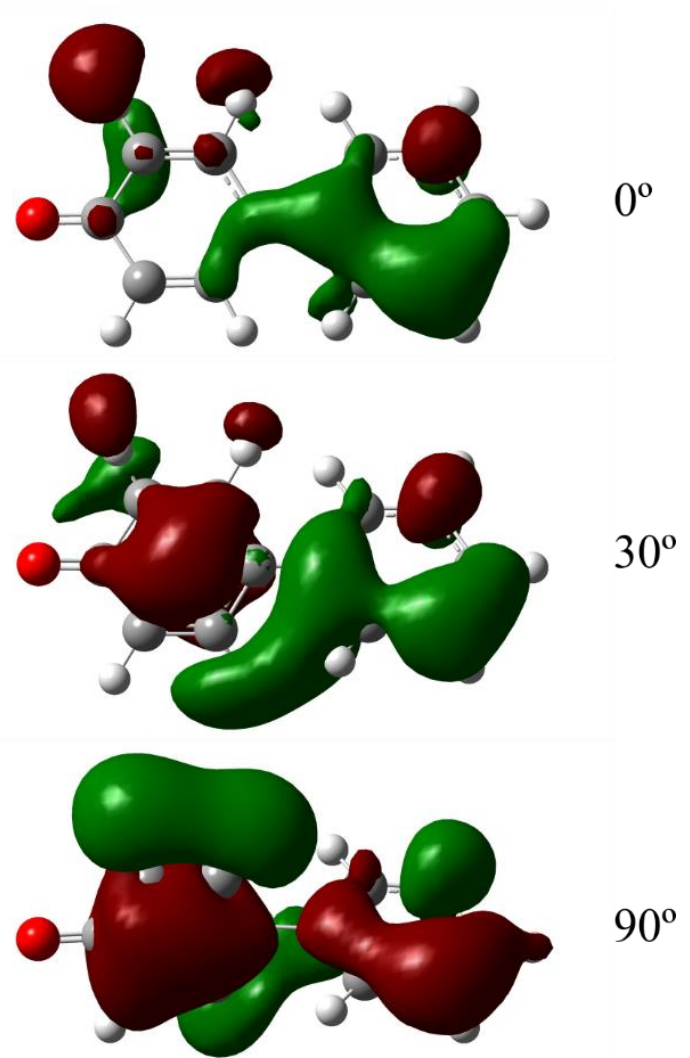

Figura 14. Orbitais moleculares envolvidos na excitação eletrônica da pBT obtidos quando $\phi$ assume os valores de 0 , 30 e $90^{\circ}$.

Na Figura 16 vemos a competição entre os efeitos estruturais e eletrônicos devido à aplicação do campo elétrico externo F. Com o aumento da intensidade do campo F os valores de $\phi$ aumentam de $36^{\circ}$ para $43^{\circ}$ e de $32^{\circ}$ para $54^{\circ}$, respectivamente, para a oBT e para a pBT. Em geral, os resultados obtidos na condição $\mathrm{G}_{\mathrm{F}} / \mathrm{nF}$ mostram a mesma dependência estrutural observada ao induzir diferentes valores de $\phi$. Esse comportamento similar era esperado pois a condição $\mathrm{G}_{\mathrm{F}} / \mathrm{nF}$ considera a influência do campo $\mathrm{F}$ somente para obtenção das geometrias de mínima energia em cada valor de $\mathrm{F}$ enquanto as propriedades eletrônicas foram obtidas para as moléculas isoladas $(\mathrm{F}=0)$. 

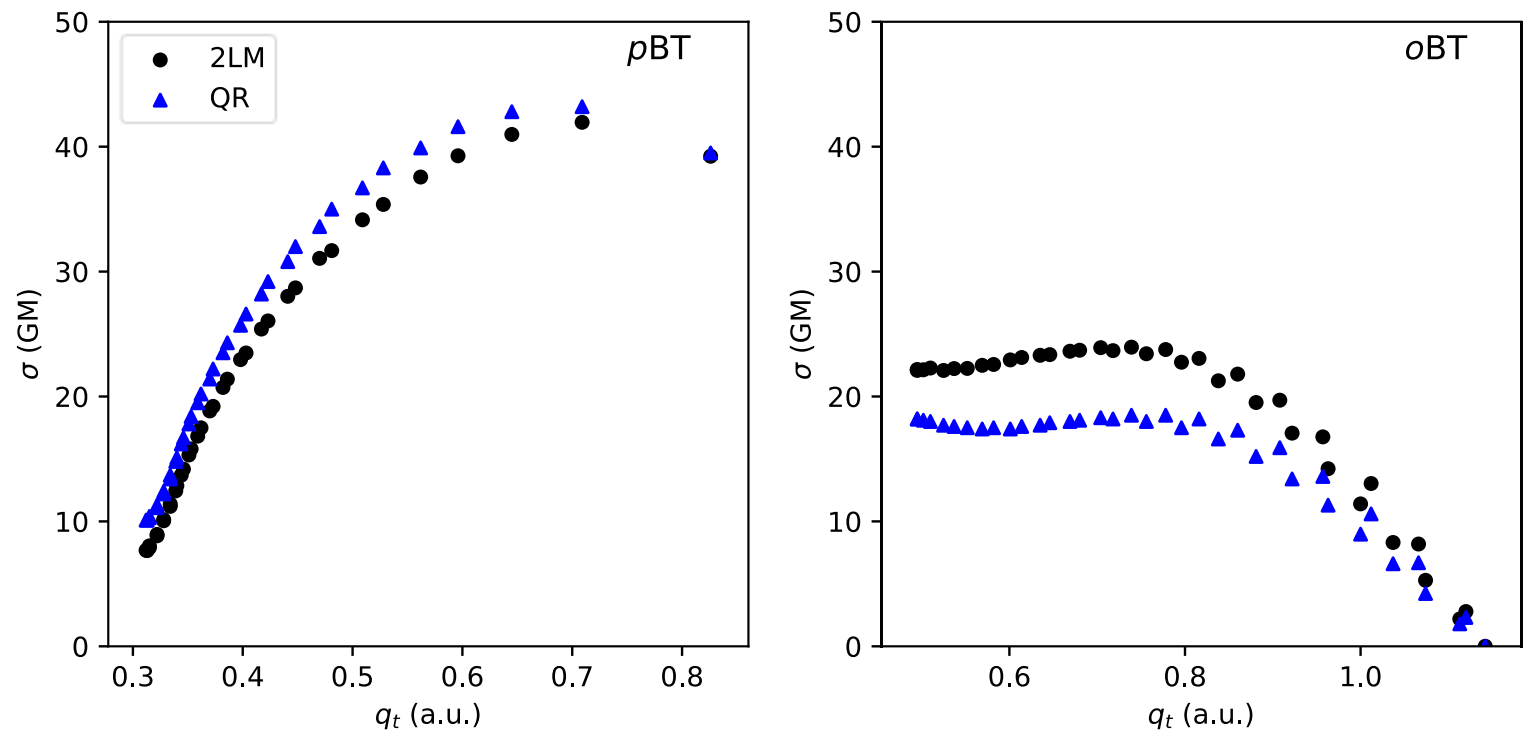

Figura 15. Seção de choque de absorção de dois fótons $\sigma$ obtida utilizando o modelo de dois níveis e teoria de resposta quadrática em função da carga qt transferida durante a excitação eletrônica.

Analisamos as propriedades eletrônicas envolvidas no processo de A2F, mais especificamente as propriedades envolvidas na aproximação 2LM, para diferentes valores de F e observamos algumas características interessantes para $\mathrm{G}_{\mathrm{F}} / \mathrm{wF}$ e $\mathrm{G}_{\mathrm{R}} / \mathrm{wF}$. Para a energia de transição eletrônica, claramente se observa uma competição entre os efeitos geométricos (redução de $\Delta E$ ) e os efeitos devidos à presença do campo $\mathrm{F}$ (aumento de $\Delta E$ ) para a $\mathrm{pBT}$. Quando $\mathrm{F}>0.0105$ u.a. os maiores valores observados para $\Delta E$ foram obtidos com $\mathrm{G}_{\mathrm{F}} / \mathrm{wF}$ enquanto para valores inferiores de $F$ a condição $G_{R} / w F$ apresentava maiores valores. Observamos essa inversão somente para a pBT enquanto a oBT apresenta um comportamento quase linear. Entretanto, o comportamento do momento de dipolo permanente do estado excitado da molécula pBT é o mais intrigante. Enquanto os valores obtidos considerando $\mathrm{G}_{\mathrm{R}} / \mathrm{wF}$ apresenta um comportamento crescente com o aumento de $\mathrm{F}$, os valores obtidos considerando $\mathrm{G}_{\mathrm{F}} / \mathrm{wF}$ são relativamente constantes para $45<\mathrm{F}<100\left(10^{-4}\right.$ u.a. $)$. A presença do campo $\mathrm{F}$ aumenta a separação de carga do estado fundamental e diminui o momento de dipolo de transição $\mu^{g e}$ como consequência da redução da mobilidade eletrônica. Esse comportamento é observado nas três condições simuladas e é mais evidente para os resultados obtidos com $\mathrm{G}_{\mathrm{F}} / \mathrm{wF}$. 

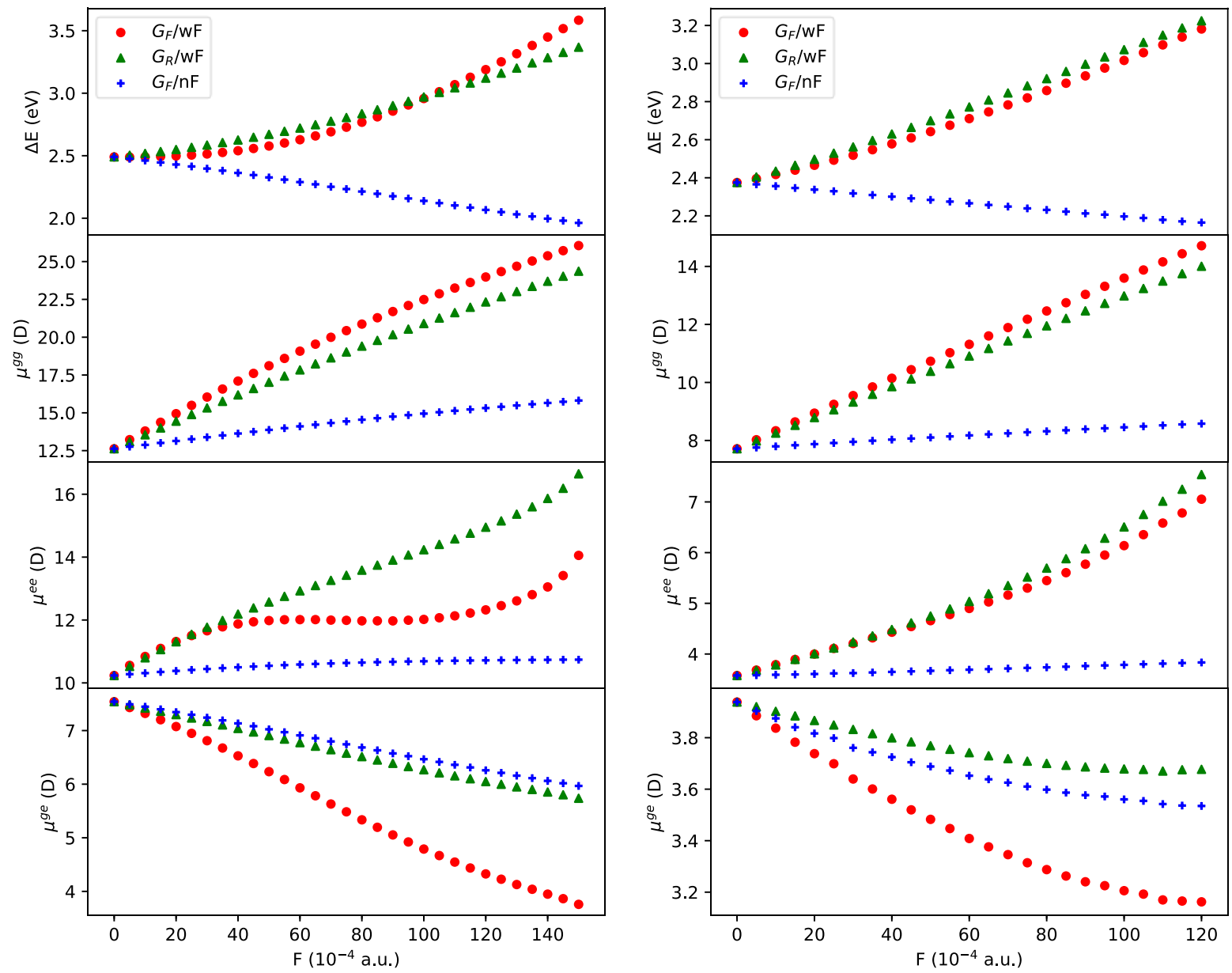

Figura 16. Energia de transição $\Delta E$, momento de dipolo permanente do estado fundamental $\mu^{g g}$ e do estado excitado $\mu^{e e} e$ momento de dipolo de transição entre o estado fundamental e excitado $\mu^{\text {ge }}$ obtidos para a pBT (esquerda) e para a oBT (direita) em diferentes valores de intensidade de campo elétrico. $G_{F}$ : Geometria obtida incluindo efeitos do campo $F$; $G_{R}$ : Geometria obtida na condição de molécula isolada; wF: Propriedades eletrônicas obtidas considerando o campo F; $n F$ : Propriedades eletrônicas obtidas na condição de molécula isolada.

$\mathrm{O}$ aumento da intensidade do campo $\mathrm{F}$ aumenta a polarização eletrônica induzindo maior separação de cargas e consequentemente a distância $\mathrm{R}$ da carga transferida durante a excitação é maior. Como a densidade eletrônica está mais concentrada nas extremidades, são esperados maiores valores de $\mathrm{q}_{\mathrm{t}}$ com o aumento de F. Como consequência desses dois comportamentos, a probabilidade de $\mathrm{A} 1 \mathrm{~F}$, relacionada $\operatorname{com} \mu^{g e}$, é reduzida devido à dificuldade de movimentar essas cargas. A presença do campo elétrico estático também restringe a oscilação da nuvem eletrônica contribuindo na redução da probabilidade de absorção. Novamente, o comportamento de $R$ e de $q_{t}$ em função de variações da geometria $\left(\mathrm{G}_{\mathrm{F}} / \mathrm{nF}\right)$ mostram uma tendência quase linear como observado na análise em função do ângulo $\phi$. Quando o campo F é aplicado na geometria dos compostos isolados $\left(\mathrm{G}_{\mathrm{R}} / \mathrm{wF}\right)$ vemos um aumento em $\mathrm{q}_{\mathrm{t}}$ em relação aos resultados obtidos para $\mathrm{G}_{\mathrm{F}} / \mathrm{nF}$. Os valores obtidos para $\mathrm{q}_{\mathrm{t}}$ são ainda maiores quando ambos os efeitos, geométricos e eletrônicos, devido à presença de $\mathrm{F}$ são considerados. Além disso, observamos que os efeitos geométricos aumentam $\mathrm{q}_{\mathrm{t}}$ em pouco mais 
de $0.1 e$ indicando maior sensibilidade devido à presença de $\mathrm{F}$. Os resultados estão apresentados na Figura 17. Podemos ver que o aumento da distância R, devido à perturbação de $\mathrm{F}$, leva o centro de decréscimo de carga na direção do oxigênio ao mesmo tempo que leva o centro de acréscimo de carga para além do nitrogênio. Os centros de acréscimo e decréscimo de carga durante a transição eletrônica e a variação positiva $\left(\rho_{+}\right.$, roxo $)$e negativa $\left(\rho_{-}\right.$, ciano) da densidade de carga induzida podem ser observadas na Figura 18 para a pBT em diferentes amplitudes de F.
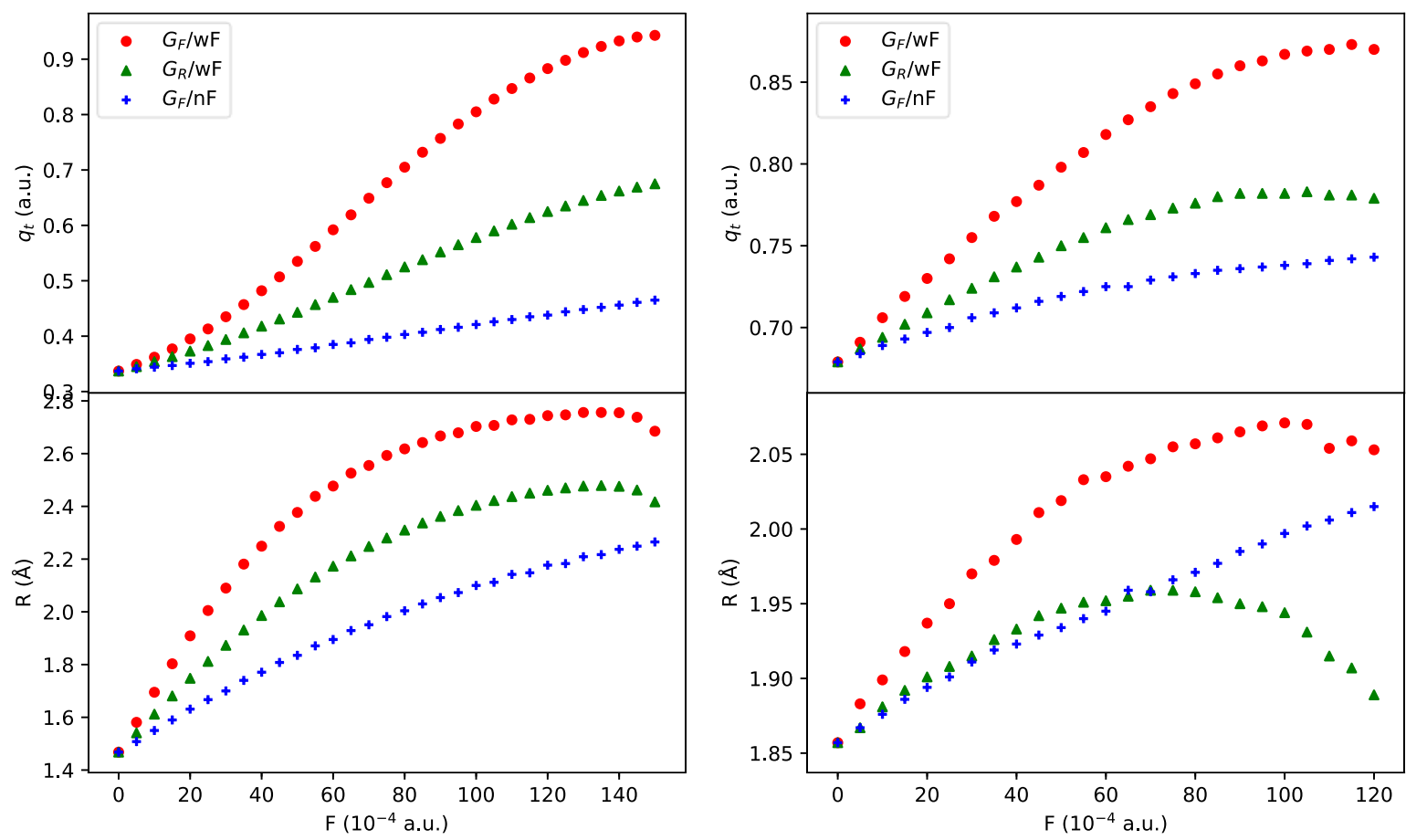

Figura 17. Quantidade de carga transferida durante a excitação $q_{t}$ e distância de deslocamento $R$ entre os pontos de decréscimo e acréscimo da carga obtidas para a pBT (esquerda) e para a oBT (direita) em diferentes valores de intensidade de campo elétrico. $G_{F}$ : Geometria obtida incluindo efeitos do campo $F$; $G_{R}$ : Geometria obtida na condição de molécula isolada; $w F$ : Propriedades eletrônicas obtidas considerando o campo $F ; n F$ : Propriedades eletrônicas obtidas na condição de molécula isolada.

Vemos na Figura 19 que F modula a amplitude da seção de choque de A2F. O efeito do campo elétrico é mais efetivo em $\mathrm{G}_{\mathrm{F}} / \mathrm{wF}$, como esperado, embora também apresente um efeito relevante quando desconsiderado o efeito estrutural $\left(\mathrm{G}_{\mathrm{R}} / \mathrm{wF}\right)$. Os valores obtidos para a seção de choque de $\mathrm{A} 2 \mathrm{~F}$ em função de $\mathrm{F}$ apresentam grande aumento até atingir um ponto máximo, a partir de então a interação com o campo elétrico externo passa a contribuir negativamente. Esse comportamento é observado tanto para pBT quanto para a oBT. Em números, obtidos utilizando $\mathrm{QR}$, vemos um aumento de $\sigma(0)=16$ para valor máximo de $\sigma(100)=87$ GM para a pBT considerando $\mathrm{G}_{\mathrm{F}} / \mathrm{wF}$. Para a oBT esse aumento é menos expressivo, indo de $\sigma(0)=18$ para $\sigma(75)=22$ GM. A aproximação 2LM para a pBT deslocou o valor máximo de $\sigma$ para maiores amplitudes de F além de apresentar maiores valores. Analisando estes resultados, podemos 
compreender o valor máximo de $\sigma$ como a combinação de dois fatores: (i) a retomada do crescimento do momento de dipolo permanente do estado excitado após $\mathrm{F}=100$ ( $10^{-4}$ u.a.), reduzindo a diferença entre os momentos de dipolo permanente; e (ii) o decréscimo do momento de dipolo de transição.
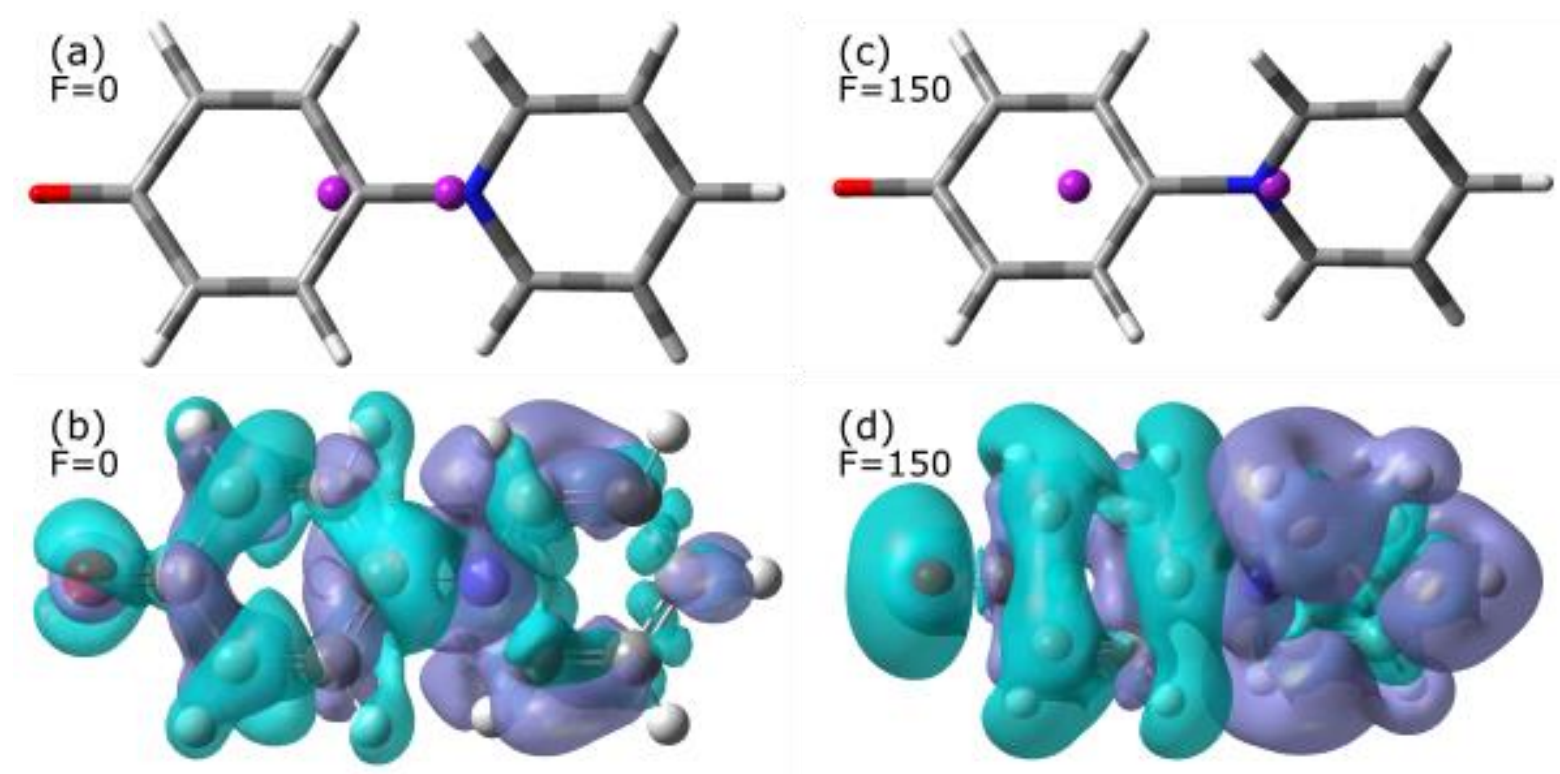

Figura 18. Centros de decréscimo e acréscimo de cargas (superior) e diferença das densidades eletrônicas entre os estados fundamental e excitado (inferior) para a pBT. A cor ciano representa a densidade negativa e a roxa a densidade positiva. A unidade de $\mathrm{F}$ é $10^{-4}$ u.a.

Seguindo para descrições mais realistas de solventes, consideramos a aproximação PCM para solventes de diversas polaridades (utilizamos como base a escala de polaridade $E_{T}^{N}$ proposta por Reichardt [156]). Os resultados estão sumarizados na Tabela 7. É conhecido que a geometria de mínima energia do composto oBT em água não é bem descrita utilizando a aproximação PCM [153]. Considerando o esforço computacional para obter tais geometrias em diversos solventes e que a descrição estrutural foge ao escopo deste trabalho, optamos por estudar somente a pBT com PCM.

Os comprimentos de ligação entre os anéis $\left(\mathrm{R}_{\mathrm{NC}}\right)$ e entre o oxigênio e o anel $\left(\mathrm{R}_{\mathrm{CO}}\right)$, o ângulo torcional $(\phi)$ e as propriedades eletrônicas relacionadas com o processo de A2F da pBT foram analisados em 12 solventes aproximados com PCM. Observamos poucas alterações dos valores obtidos, principalmente para fatores estruturais $\mathrm{R}_{\mathrm{NC}}, \mathrm{R}_{\mathrm{CO}}$ e $\phi$. Além disso, tivemos dificuldade em reproduzir os resultados experimentais [157] para as energias de transição. Os valores das diferenças entre os resultados teóricos e experimentais não foram sistemáticos e não descrevem corretamente os efeitos solvatocrômicos da pBT. Estas diferenças das energias de transição, entre os resultados teóricos e experimentais, chegaram a valores de $-0.52 \mathrm{eV}$ para água e $+0.31 \mathrm{eV}$ para dimetilsulfóxido. Contudo, os solventes clorofórmio, benzeno e tolueno 
apresentaram valores de $\mathrm{q}_{\mathrm{t}} \mathrm{e} \mathrm{R}$ distintos dos demais solventes, indicando baixa polarização eletrônica, como esperado para solventes de baixa polaridade.
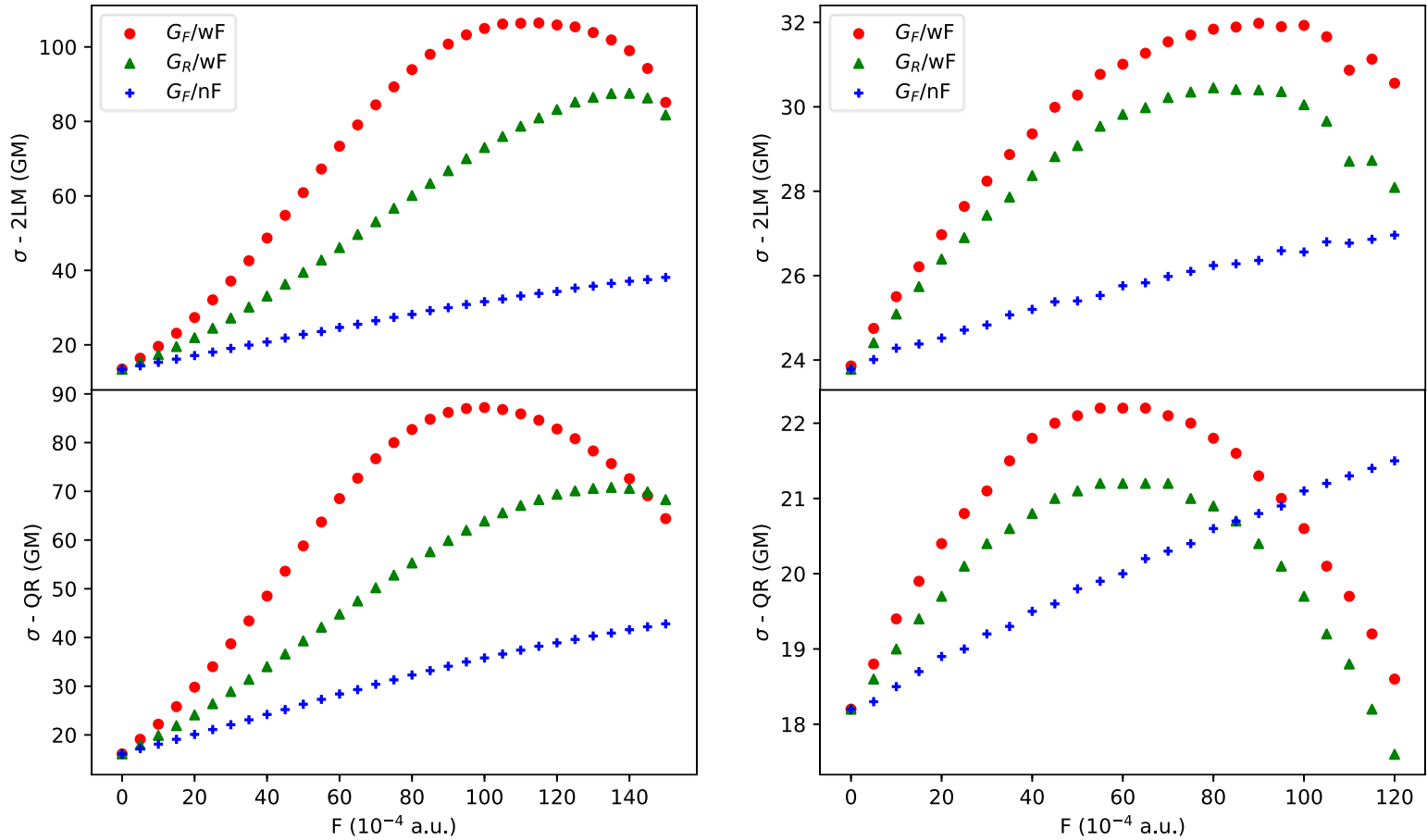

Figura 19. Seção de choque de A2F obtidas para a pBT (esquerda) e para a oBT (direita) em diferentes valores de intensidade de campo elétrico. $G_{F}$ : Geometria obtida incluindo efeitos do campo $F$; $G_{R}$ : Geometria obtida na condição de molécula isolada; wF: Propriedades eletrônicas obtidas considerando o campo F; $n F$ : Propriedades eletrônicas obtidas na condição de molécula isolada.

Em todos os solventes os valores obtidos para $\sigma$ utilizando 2LM foram maiores que os obtidos utilizando o formalismo $\mathrm{QR}$, sendo essas diferenças mais expressivas para solventes de maior polaridade. Apesar dessa diferença, esse modelo simplificado é útil para auxiliar no entendimento dos altos valores de $\sigma$ obtidos, comparativamente com os valores obtidos para os compostos isolados e utilizando aplicações de campo elétrico. Comparamos os valores obtidos em água, utilizando PCM, com os valores obtidos com $\mathrm{F}=85 \times 10^{-4}$ u.a. (por apresentar o ângulo $\phi=45.2^{\circ}$ e energia de transição $\Delta E=2.81 \mathrm{eV}$ semelhante aos valores obtidos com PCM). A diferença na descrição do momento de dipolo permanente do estado excitado é o fator predominante neste grande aumento de $\sigma$. O valor do $\mu^{e e}$ obtido com PCM foi de $6.80 \mathrm{D}$ enquanto o valor obtido com $\mathrm{F}=85 \times 10^{-4}$ u.a foi de $11.87 \mathrm{D}$. O valor obtido para $\mu^{g e}$ também é um fator que aumenta os valores de $\sigma$ obtidos com PCM. Essa análise está apresentada na Tabela 8. 
Tabela 7. Variação das propriedades eletrônicas relacionadas com o processo de AlF e de A2F da pBT considerando a aproximação PCM. *Entre parênteses apresentamos resultados experimentais [157], ins = insolúvel.

\begin{tabular}{|c|c|c|c|c|c|c|}
\hline Solvente & $E_{T}^{N}$ & $\phi\left(^{\circ}\right)$ & $\mathbf{R}_{\mathbf{N C}}(\AA)$ & $\mathbf{R}_{\mathbf{C O}}(\AA)$ & $\Delta \mathbf{E}^{*}(\mathbf{e V})$ & $\mu^{\mathrm{ge}}(\mathrm{D})$ \\
\hline Água & 1.00 & 44.58 & 1.44 & 1.29 & $2.88(3.40)$ & 6.65 \\
\hline Metanol & 0.76 & 44.27 & 1.44 & 1.29 & $2.84(3.15)$ & 6.69 \\
\hline Etanol & 0.65 & 44.08 & 1.44 & 1.29 & $2.82(3.00)$ & 6.72 \\
\hline 1-Propanol & 0.62 & 43.91 & 1.44 & 1.29 & $2.80(2.90)$ & 6.75 \\
\hline 2-Propanol & 0.55 & 43.84 & 1.44 & 1.29 & $2.79(2.81)$ & 6.75 \\
\hline Acetonitrila & 0.46 & 44.32 & 1.44 & 1.29 & $2.84(2.67)$ & 6.69 \\
\hline Dimetilsulfóxido & 0.44 & 44.45 & 1.44 & 1.29 & $2.86(2.55)$ & 6.67 \\
\hline Acetona & 0.36 & 43.91 & 1.44 & 1.29 & 2.80 (ins) & 6.75 \\
\hline Diclorometano & 0.31 & 42.57 & 1.44 & 1.29 & 2.67 (ins) & 6.95 \\
\hline Clorofórmio & 0.26 & 40.37 & 1.43 & 1.28 & 2.53 (ins) & 7.29 \\
\hline Benzeno & 0.11 & 34.69 & 1.41 & 1.27 & 2.39 (ins) & 7.95 \\
\hline Tolueno & 0.10 & 35.16 & 1.42 & 1.27 & 2.40 (ins) & 7.91 \\
\hline Solvente & $\mu^{\mathrm{gg}}(\mathrm{D})$ & $\mu^{\mathrm{ee}}(\mathrm{D})$ & $\sigma^{2 \mathrm{LM}}(\mathrm{GM})$ & $\sigma^{\mathrm{QR}}(\mathbf{G M})$ & $q_{t}$ (u.a.) & $\mathbf{R}(\AA)$ \\
\hline Água & 22.88 & 6.80 & 477.19 & 376.00 & 0.94 & 3.56 \\
\hline Metanol & 22.63 & 6.85 & 465.09 & 371.00 & 0.93 & 3.53 \\
\hline Etanol & 22.49 & 6.88 & 459.22 & 368.00 & 0.92 & 3.52 \\
\hline 1-Propanol & 22.36 & 6.92 & 452.92 & 365.00 & 0.92 & 3.50 \\
\hline 2-Propanol & 22.32 & 6.93 & 450.36 & 364.00 & 0.91 & 3.50 \\
\hline Acetonitrila & 22.66 & 6.84 & 467.45 & 372.00 & 0.93 & 3.53 \\
\hline Dimetilsulfóxido & 22.76 & 6.82 & 471.74 & 374.00 & 0.94 & 3.54 \\
\hline Acetona & 22.36 & 6.92 & 453.29 & 365.00 & 0.92 & 3.50 \\
\hline Diclorometano & 21.45 & 7.27 & 405.32 & 338.00 & 0.87 & 3.41 \\
\hline Clorofórmio & 20.02 & 8.06 & 317.24 & 277.00 & 0.77 & 3.23 \\
\hline Benzeno & 16.89 & 9.94 & 127.40 & 122.00 & 0.54 & 2.70 \\
\hline Tolueno & 17.14 & 9.82 & 139.91 & 133.00 & 0.55 & 2.75 \\
\hline
\end{tabular}

Uma forma iterativa de polarização do soluto em meio líquido utilizando simulações moleculares clássicas tem mostrado sucesso para melhorar a descrição de transições eletrônicas [158,159]. Para essas simulações, o campo de força da pBT foi construído utilizando os parâmetros de Lennard-Jones e o conjunto de cargas obtidas inicialmente com PCM. Ao final da simulação as propriedades eletrônicas envolvidas no processo de A2F foram obtidas considerando a aproximação ASEC proveniente da simulação molecular, esses resultados estão identificados como ASEC-0. Em seguida uma nova simulação foi realizada considerando o conjunto de cargas obtidas com ASEC-0. Novamente as propriedades eletrônicas foram obtidas ao final da simulação, identificado como ASEC-1. Essa atualização das cargas moleculares, nova simulação molecular e subsequentes cálculos da A2F foram realizadas mais uma vez, os 
resultados estão identificados como ASEC-2. Todas as simulações utilizam geometrias rígidas obtidas com PCM.

A polarização iterativa em água resultou em melhor concordância da energia de transição eletrônica da pBT com o valor experimental $(3.40 \mathrm{eV})$ devido a um deslocamento de quase $1 \mathrm{eV}$ para maiores energias. Os momentos de dipolo permanente da $\mathrm{pBT}$, tanto do estado fundamental quanto do excitado, também aumentaram quando comparados com os resultados obtidos com PCM e F $=85 \times 10^{-4}$ u.a. Todavia, o valor da diferença $\Delta \mu$ foi de aproximadamente 11.2 $\mathrm{D}$ nas três iterações, resultando em $\sigma \approx 110 \mathrm{GM}$ na aproximação $2 \mathrm{LM}$ e em $\sigma \approx 80 \mathrm{GM}$ no formalismo QR. Esses resultados corroboram a dificuldade em obter propriedades eletrônicas de estados excitados utilizando TD-DFT com PCM seja para o modelo simplificado 2LM ou para o formalismo QR. Os resultados estão apresentados na Tabela 8.

Tabela 8. Energia de transição e momento de dipolo permanente e de transição para a pBT em diferentes aproximações de solventes. $\Delta \mu=\mu^{g g}-\mu^{e e}$. ASEC- $n$ : $n$ representa o número da iteração no processo de polarização utilizando simulações moleculares, para $n=0$ as cargas utilizadas foram obtidas com a aproximação PCM. *A geometria utilizada nas simulações é a mesma que a utilizada no cálculo com PCM.

\begin{tabular}{|cccccccc|}
\hline & $\begin{array}{c}\Delta \mathbf{E} \\
(\mathbf{e V})\end{array}$ & $\begin{array}{c}\boldsymbol{\mu}^{\mathbf{g g}} \\
(\mathbf{D})\end{array}$ & $\begin{array}{c}\boldsymbol{\mu}^{\mathbf{e e}} \\
(\mathbf{D})\end{array}$ & $\begin{array}{c}\Delta \boldsymbol{\mu} \\
(\mathbf{D})\end{array}$ & $\begin{array}{c}\boldsymbol{\mu}^{\mathbf{g e}} \\
(\mathbf{D})\end{array}$ & $\begin{array}{c}\boldsymbol{\sigma}^{\mathbf{2 L M}} \\
(\mathbf{G M})\end{array}$ & $\begin{array}{c}\boldsymbol{\sigma}^{\mathbf{Q R}} \\
(\mathbf{G M})\end{array}$ \\
\hline F=85x10-4 (u.a.) & 2.81 & 21.29 & 11.97 & 9.31 & 5.20 & 98.02 & 84.80 \\
Água - PCM & 2.88 & 22.88 & 6.80 & 16.08 & 6.65 & 477.19 & 376.00 \\
Água - ASEC-0 & 3.44 & 25.55 & 14.34 & 11.21 & 4.59 & 110.35 & 84.40 \\
Água - ASEC-1 & 3.60 & 26.25 & 15.07 & 11.18 & 4.50 & 108.30 & 80.40 \\
Água - ASEC-2 & 3.64 & 26.46 & 15.27 & 11.19 & 4.49 & 107.27 & 79.00 \\
\hline
\end{tabular}

\subsubsection{Conclusões}

Neste trabalho estudamos os processos de A1F e de A2F dos compostos pBT e oBT analisando variações do ângulo torcional $(\phi)$, considerando perturbações externas causadas por campos elétricos estáticos e efeitos de solventes. Este trabalho é uma extensão de estudos prévios realizados para a pBT em função de $\phi$ e vem a contribuir para a descrição de efeitos estruturais e de solventes sobre o processo de A2F.

Mostramos que as variações do ângulo torcional da pBT e da oBT resultaram em comportamentos iguais para o processo de $\mathrm{A} 1 \mathrm{~F}$ e diferentes para o processo de $\mathrm{A} 2 \mathrm{~F}$. Ambos os compostos apresentaram redução da probabilidade de $\mathrm{A} 1 \mathrm{~F}$ com o aumento de $\phi$ e tal comportamento levou a anulação da A1F para a oBT. Já para o processo de A2F, observamos maior sensibilidade em função de $\phi$ para a pBT com um aumento de 4 vezes conforme o aumento de $\phi$, enquanto a oBT apresentou valores praticamente constantes para o processo de 
A2F até $\phi=60^{\circ}$, a partir do qual um decréscimo acentuado é observado até a intensidade do processo se anular em $\phi=90^{\circ}$.

A representação do solvente com PCM apresentou dificuldades para descrever o momento de dipolo permanente do estado excitado, levando a intensidades de A2F muito elevadas comparativamente com os demais resultados. Por outro lado, a utilização de um banho eletrostático médio com ASEC melhorou a descrição tanto da energia de transição quanto da intensidade de A2F. Assim, a utilização do ASEC para obter a polarização das cargas é uma alternativa interessante para descrever a intensidade da seção de choque de A2F.

A utilização de campos elétricos externos de diferentes intensidades pode ser empregada como modelo para avaliar os efeitos de interações eletrostáticas com o meio solvente. Com o aumento do campo $\mathrm{F}$ observamos um decréscimo da A1F e opostamente observamos um aumento da A2F. Portanto, tal observação nos permite concluir que é possível utilizar de campos elétricos externos para modular a intensidade da $\mathrm{A} 2 \mathrm{~F}$ ao mesmo tempo que o processo de A1F é inibido. Esse controle foi mais efetivo para o composto pBT. Uma vez que espectros de A2F podem ser inconvenientemente alterados devido a efeitos de engrandecimento por A1F, a utilização de campos elétricos para reduzir estes efeitos é uma proposta ainda a ser verificada experimentalmente. Também mostramos, de forma semi quantitativa, que maiores quantidades de carga eletrônica transferida durante a excitação e deslocadas por maiores distâncias levam à maiores valores da seção de choque de A2F.

\subsection{Espectro de absorção de dois fótons de sistemas moleculares grandes}

\subsubsection{Introdução}

No ano de 2005, foi sintetizado um conjunto de moléculas orgânicas cuja fluorescência se encontra na região de $450 \mathrm{~nm}$ [160]. Posteriormente, buscando melhores solubilidades em solventes orgânicos, novos compostos foram sintetizados incluindo cadeias hidrofílicas [161] e dois deles apresentaram altos valores para as seções de choque de A2F ( 400 GM). A solubilidade em água e alta intensidade de A2F indicam potencial aplicações em imagiamento biológico, o que de fato foi verificado [48]. A principal diferença estrutural entre estes dois novos compostos é a existência de estruturas carbônicas para conectar dois anéis presentes na região central de modo a limitar a flexibilidade no núcleo aromático. Utilizaremos a mesma notação que os trabalhos anteriores: 7 para o composto com uma ligação simples entre os anéis 
centrais (azul) e $\mathbf{1 0}$ para o composto com flexibilidade limitada (vermelho), as estruturas químicas desses compostos estão apresentadas na Figura 20.

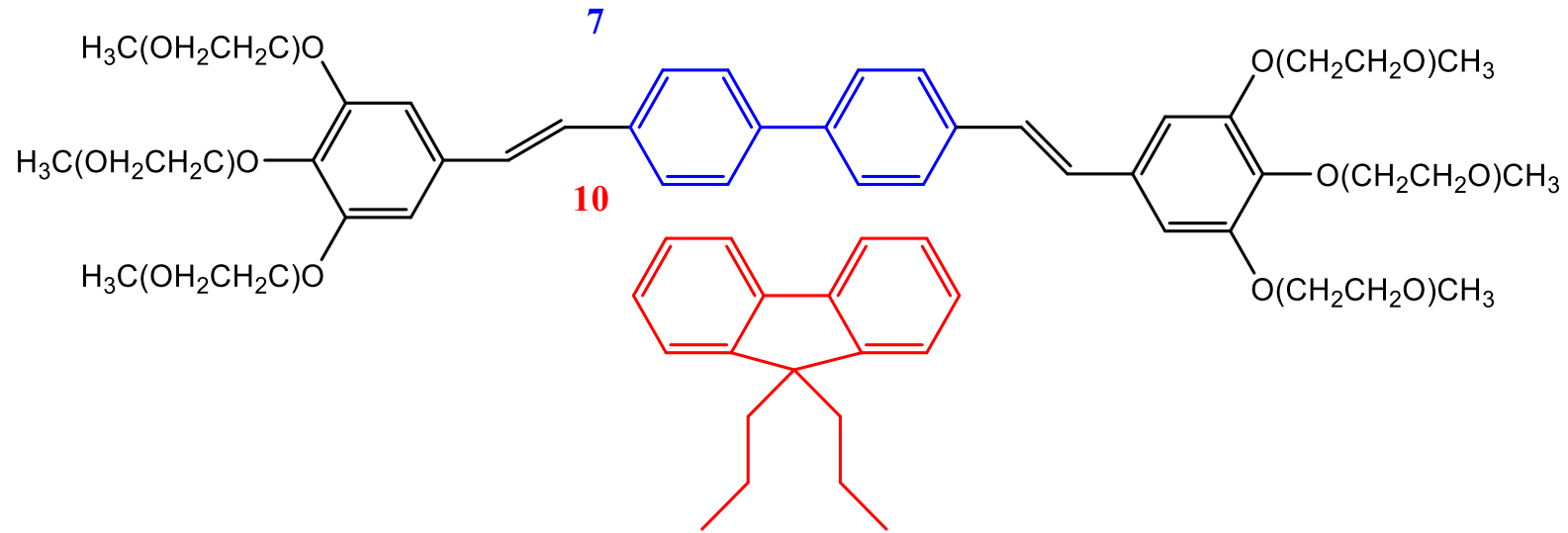

Figura 20. Estrutura química dos compostos 7 (em azul) e 10 (em vermelho) [160,161].

Os valores experimentais da seção de choque de $\mathrm{A} 2 \mathrm{~F}$ foram obtidos utilizando a técnica de fluorescência por excitação de dois fótons. Para isso os autores utilizaram pulsos de $2.6 \mathrm{~ns}$ (Nd:YAG) na região de 450-650 nm e pulsos de femtosegundos (Ti:safira) na região de 700$900 \mathrm{~nm}$. Os valores máximos da seção de choque de A2F obtidos na região de $520 \mathrm{~nm}$ atingem a magnitude de 400 GM [161], evidenciando grande potencial para aplicações diretas. Contudo, devido à combinação dos lasers utilizados no experimento, a região entre 650-720 nm não pode ser caracterizada. Nessa lacuna do espectro existe a expectativa de existência de uma banda de A2F (veja Figura 21) a qual poderia aumentar a eficiência do processo de absorção em energias mais baixas. Neste cenário, a realização de cálculos teóricos é uma alternativa para complementar e descrever este espectro, principalmente na região não caracterizada experimentalmente.

Descrever teoricamente o espectro de A2F dos compostos $\mathbf{7}$ e $\mathbf{1 0}$ é nosso principal objetivo neste estudo. Para isso, investigamos as bandas de absorção dando maior ênfase na região ausente do espectro experimental. A intensidade relativa, as transições eletrônicas e a influência das cadeias hidrofílicas na composição das bandas de absorção também foram discutidas. De forma complementar investigamos o processo de A1F. 


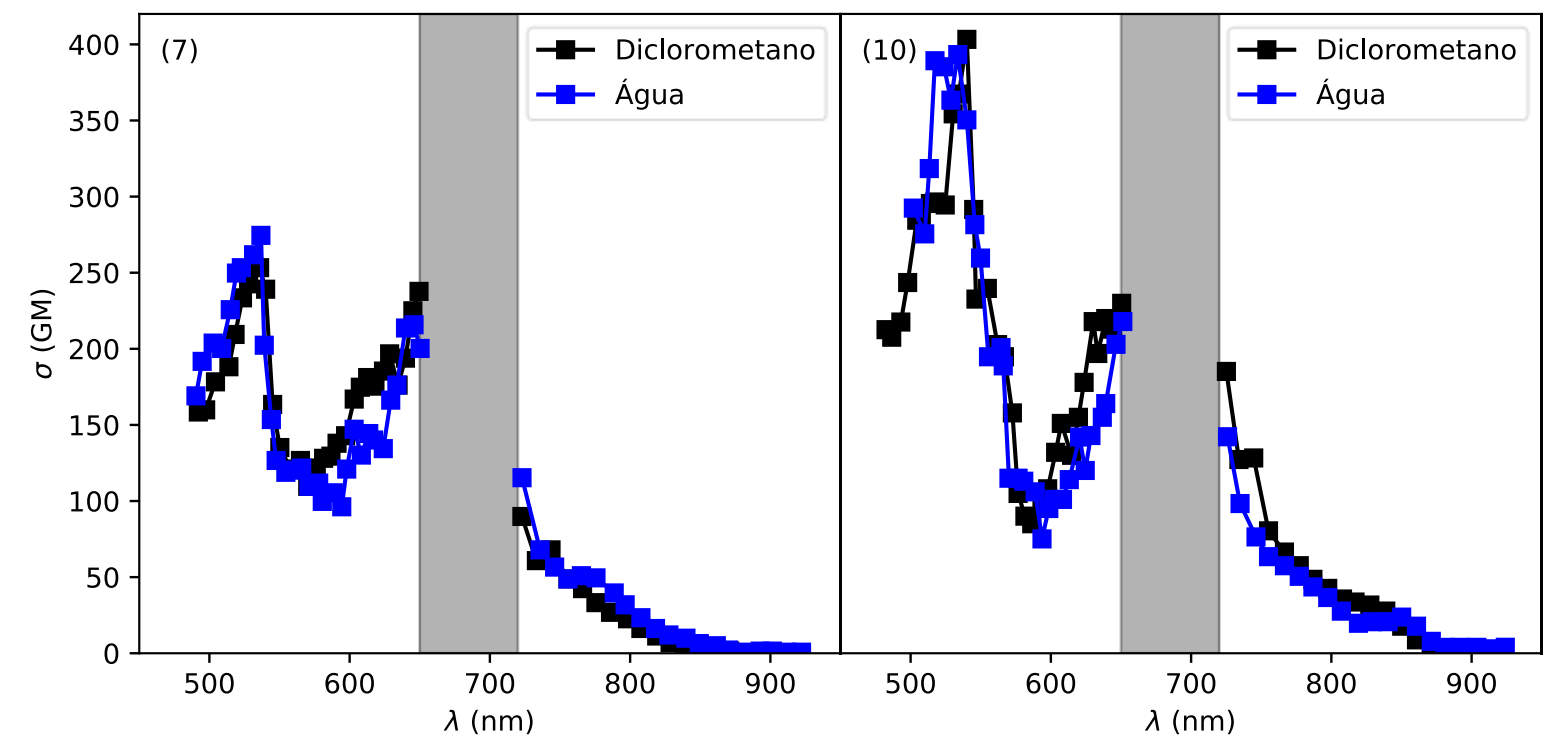

Figura 21. Seção de choque de A2F. Adaptado da referência [161].

\subsubsection{Detalhes computacionais}

Cálculos de otimização de geometria foram realizados para obter geometrias moleculares que representem condições de mínimos na superfície de energia para os compostos isolados e considerando a aproximação PCM para o solvente. Verificamos a condição de mínimo analisando o sinal das frequências vibracionais harmônicas dos compostos. Esses cálculos foram realizados no programa Gaussian 09 [81] utilizando o funcional DFT híbrido B3LYP e o conjunto de funções bases 6-31G(d).

Obtivemos as energias e as intensidades de transição, utilizando o formalismo DFT dependente do tempo com os funcionais B3LYP, BHandHLYP e CAM-B3LYP, para as geometrias moleculares de mínima energia. Uma vez que diferentes funcionais DFT se baseiam em distintas aproximações para as energias de troca e correlação, optamos por esses três funcionais para observar os efeitos dessas contribuições nos espectros. Utilizamos o conjunto de funções bases 6-31+G(d) em todos esses cálculos de absorção eletrônica.

Também utilizamos o método semi-empírico INDO/S implementado no programa ZINDO [162] utilizando excitações simples e duplas, respectivamente identificados no decorrer do texto como ZINDO/S e ZINDO/SD. Para computar as funções de onda dos estados excitados é necessário definir um espaço ativo para as excitações simples e, quando necessário, para as excitações duplas. Definimos o número OC de orbitais ocupados assumindo um número mínimo de 30 orbitais com o vínculo de que a diferença de energia entre os orbitais HOMO$(\mathrm{OC}-1)$ - HOMO-(OC) > $0.1 \mathrm{eV}$. Para definir o número NO de orbitais não ocupados assumimos $\mathrm{NO} \geq \mathrm{OC}$ e a diferença de energia entre os orbitais LUMO-(NO-1) - LUMO-(NO) 
$>0.1 \mathrm{eV}$. Para as excitações duplas foram incluídos os dois orbitais ocupados de maior energia e os dois orbitais não ocupados de menor energia. Na Figura 22, ilustramos a escolha do espaço ativo das excitações no diagrama de energia dos orbitais moleculares do composto $\mathbf{1 0}$. Os orbitais incluídos nas excitações simples foram coloridos em azul e os orbitais incluídos nas excitações duplas foram coloridos em vermelho.

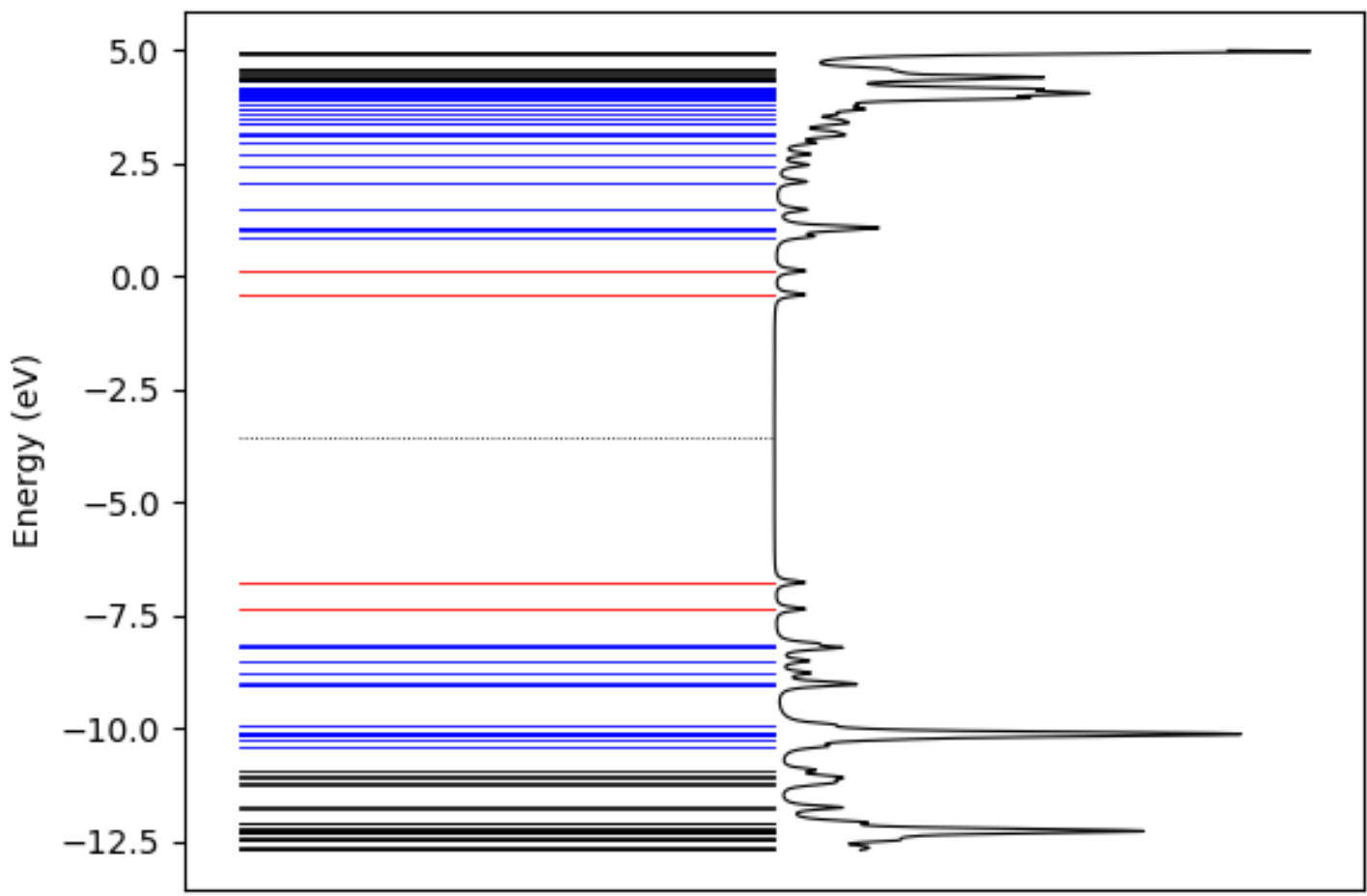

Figura 22. Diagrama de energia dos orbitais moleculares do composto 10. As cores indicam a energia dos orbitais moleculares: vermelho - incluídos nos cálculos com excitações duplas, azuis - incluídos nos cálculos com excitações simples e pretas somente por referência.

Obtivemos a seção de choque de A2F utilizando: 1) a resposta quadrática no formalismo TD-DFT com os funcionais B3LYP, BHandHLYP e CAM-B3LYP e conjunto de funções base 6-31+G(d). Todos os cálculos foram realizados utilizando o programa Dalton 2016.2 [139,140]; e 2) a soma-sobre-estados proposta por Orr [54] para computar a parte imaginária de $\boldsymbol{\gamma}$ realizada no programa PHOTON2 [163]. As energias de transição, os momentos de dipolo permanentes e os momentos de dipolo de transição para 200 estados excitados foram obtidos utilizando o programa ZINDO considerando excitações simples e duplas.

\subsubsection{Discussões}

As imagens representativas das geometrias de mínima energia estão apresentadas na Figura 23. O valor obtido para ângulo de diedro da região central do composto 7 é de $35^{\circ}$. O composto 10 apresenta uma estrutura conectando os dois anéis centrais, evitando possíveis rotações (ângulos de torções) entre estes grupos. 

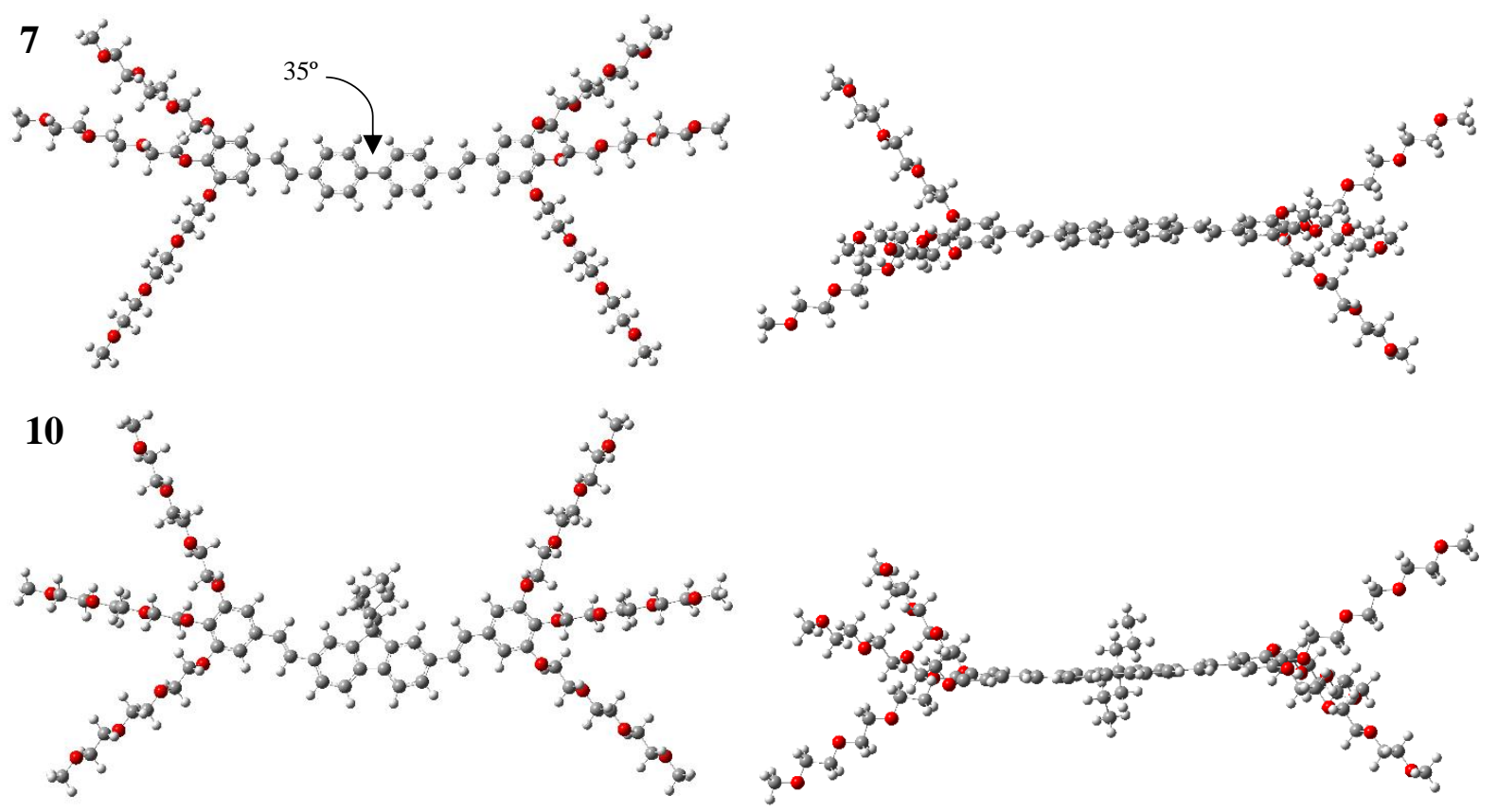

Figura 23. Geometrias de mínima energia obtidas com B3LYP/6-31G(d) para os compostos isolados.

Para complementar a análise infravermelho vibracional (IR), também calculamos o espectro Raman. Pequenas diferenças estruturais foram observadas entre as geometrias dos dois compostos e como consequência dessa similaridade os resultados dos espectros vibracionais obtidos também foram similares. Os espectros apresentados na Figura 24 foram obtidos convoluindo Lorentizianas de larguras de linha de $10 \mathrm{~cm}^{-1}$. Nossas análises mostraram duas regiões de grande intensidade. A primeira delas no espectro infravermelho na região de $1200 \mathrm{~cm}^{-1}$, relacionada com um estiramento assimétrico dos oxigênios das cadeias hidrofílicas. A segunda, no espectro Raman na região de $1650 \mathrm{~cm}^{-1}$, relacionada com uma vibração simétrica dos carbonos envolvendo os quatro anéis e as cadeias que os unem.

Grande parte do nosso estudo relacionado com os processos ópticos de A1F e A2F foi realizado considerando os compostos isolados, devido, principalmente, à similaridade dos espectros de A1F e A2F em diferentes solventes (ver Figura 21). Observamos que os orbitais moleculares envolvidos nas transições eletrônicas intensas de baixa energia não difundem sobre as cadeias hidrofílicas, sendo, portanto, o papel de tais cadeias apenas aprimorar a solubilidade molecular dos compostos. Assim, a fim de reduzir a demanda computacional nos cálculos de estrutura eletrônica, uma escolha natural é remover as cadeias hidrofílicas das geometrias de mínima energia nos cálculos. Por essa razão, introduzimos aqui a nomenclatura "completo" quando consideramos as cadeias hidrofílicas e "reduzido" quando não as consideramos (ver Figura 25) nos cálculos de estrutura eletrônica. A validade dessa aproximação será avaliada 
comparando os espectros teóricos de absorção de A1F e A2F simulados para os compostos completo e reduzido.

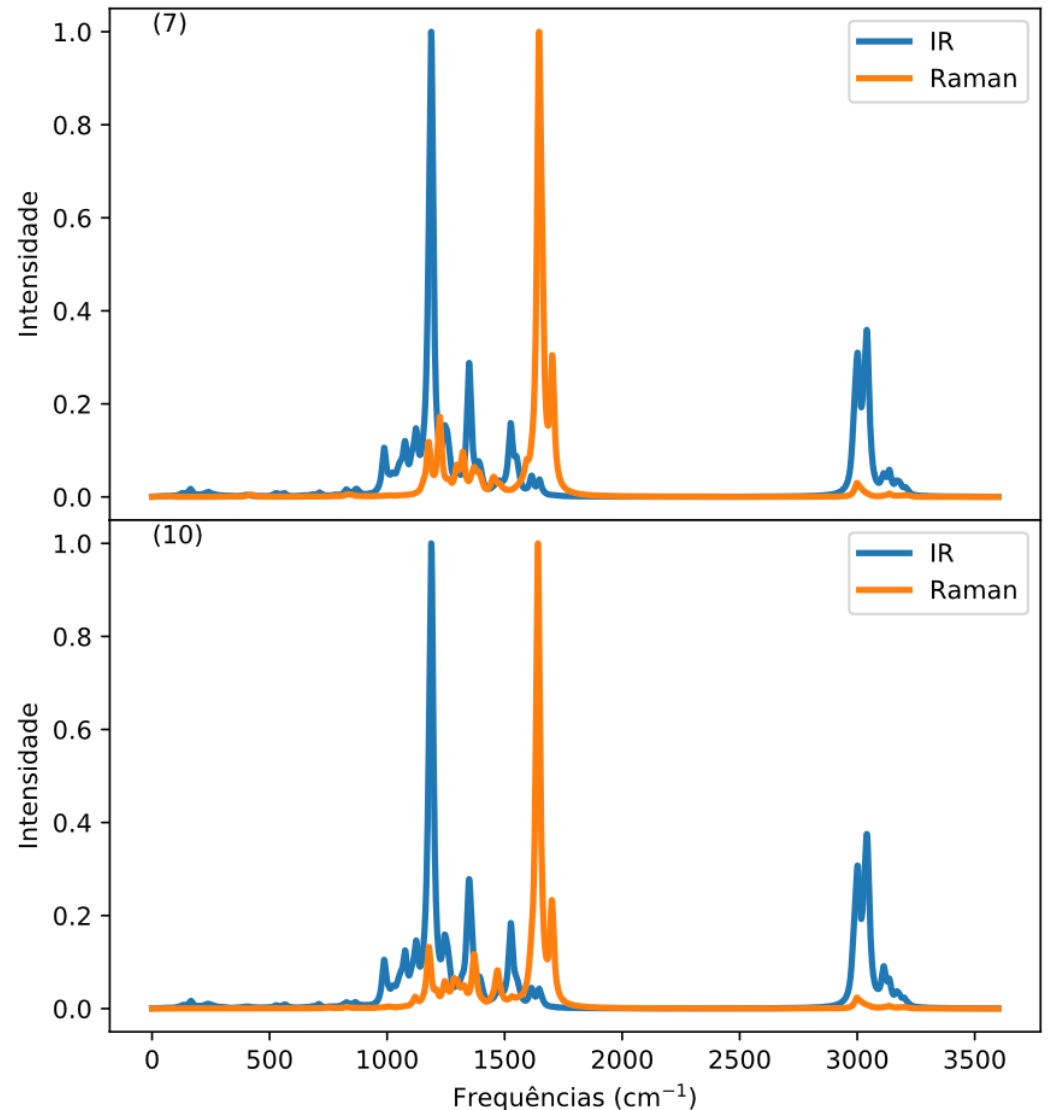

Figura 24. Espectros vibracionais infravermelho (IR) e Raman obtidos com B3LYP/6-31G(d) para os compostos isolados.

Para todos os casos avaliados, a banda de A1F é descrita por uma única transição, sendo essa uma transição do tipo $\pi-\pi^{*}$ para o primeiro estado excitado e que envolve predominantemente os orbitais HOMO-LUMO. Todos os funcionais avaliados podem ser utilizados para descrever as características eletrônicas da transição, embora os resultados obtidos com o funcional B3LYP apresentem valores da energia de transição $0.5 \mathrm{eV}$ menor que o experimental. Também observamos uma excelente concordância das energias de transição obtidas para os compostos completos e reduzidos, com uma redução de $10 \%$ das intensidades para os compostos reduzidos.

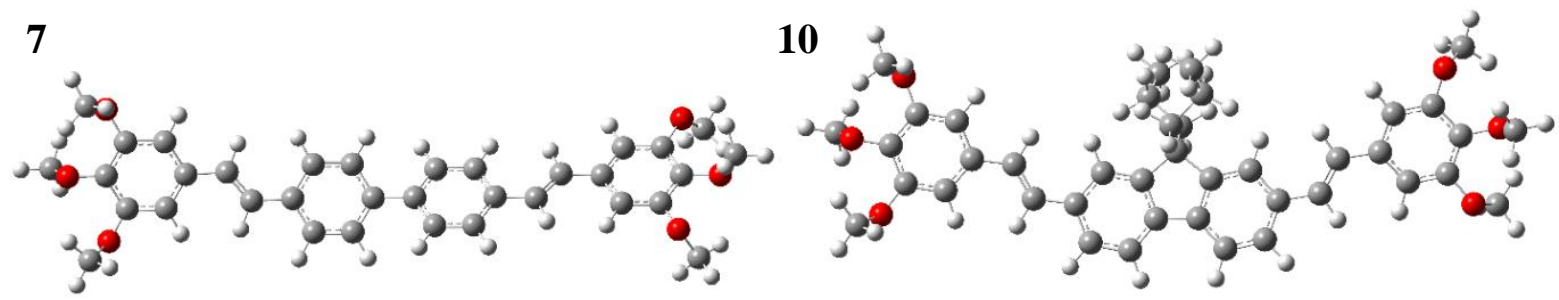


As energias de transição obtidas com o funcional CAM-B3LYP, o qual inclui correções de longo alcance, foram deslocadas em $0.5 \mathrm{eV}$ para maiores energias e tiveram um aumento da intensidade de $15 \%$, comparativamente com os resultados obtidos com o funcional B3LYP. Os resultados obtidos com o funcional BHandHLYP foram similares aos obtidos com o funcional CAM-B3LYP, indicando que maiores contribuições do termo de troca de Hartree-Fock melhoram a descrição quantitativa das energias de transição que compões o espectro de A1F. O deslocamento solvatocrômico obtido entre os compostos isolados e em água, utilizando a aproximação PCM, reduziu o valor da energia de transição em no máximo $0.09 \mathrm{eV}$, mostrando pouca dependência do espectro com o solvente e corroborado com os dados experimentais.

$\mathrm{O}$ espectro de $\mathrm{A} 1 \mathrm{~F}$ experimental para o composto $\mathbf{1 0}$ apresenta estruturas assimétricas e indica a presença de ao menos três transições nessa banda (ver Figura 26). Nossos resultados mostraram diferenças da ordem de $0.5 \mathrm{eV}$ entre a energia do primeiro e do segundo estado excitado, além disso, a transição para o segundo estado excitado não é permitida por dipolo, portanto atribuímos essa estrutura fina do espectro de $\mathrm{A} 1 \mathrm{~F}$ do composto $\mathbf{1 0}$ à efeitos vibracionais. Para essas estruturas serem avaliadas teoricamente seria necessário um estudo do espectro resolvido vibracionalmente ou combinando métodos híbridos QM/MM para amostrar diversas configurações vibracionais. Tais análises demandariam expressivo esforço computacional devido ao tamanho dos compostos e fogem ao objetivo de descrever a banda de baixa energia do espectro de A2F. Por essa razão, não analisamos tais contribuições neste trabalho.

Para construir os espectros de A1F adotamos o valor de $\Gamma=0.6 \mathrm{eV}$, equivalente à largura do espectro experimental, para as Lorentzianas. Normalizamos os espectros em função dos resultados obtidos com B3LYP para o sistema completo. Devido à semelhança entre os orbitais de Kohn-Sham, apresentamos na Figura 29 os orbitais moleculares obtidos com o funcional CAM-B3LYP. O processo de A1F, relacionado com os orbitais HOMO-LUMO segue a seta azul. Os resultados obtidos para a A1F estão sumarizados na Tabela 9 e na Figura 26. 


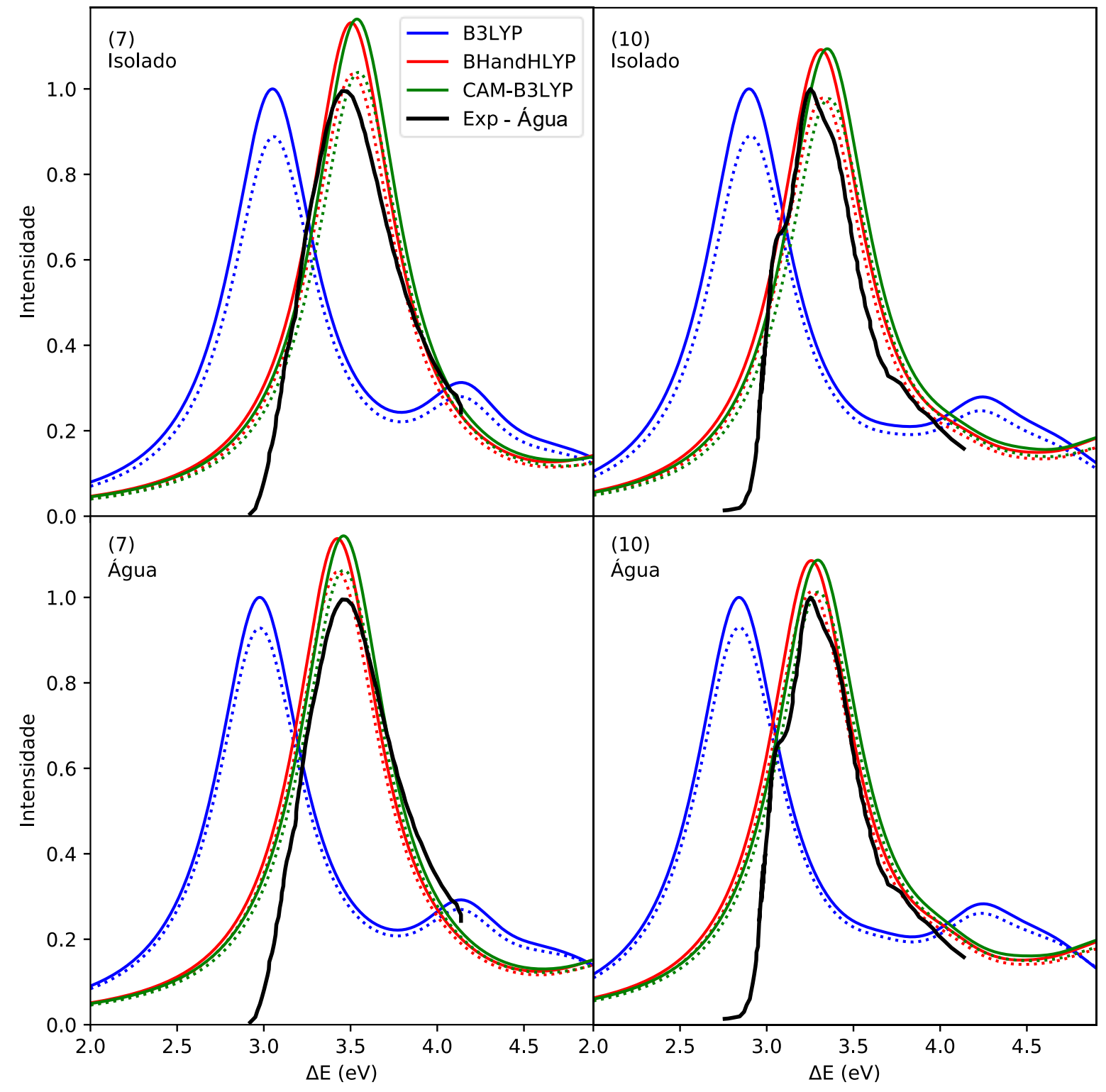

Figura 26. Espectros de AlF obtidos com diferentes funcionais DFT para os compostos completos (linhas cheias) e para os compostos reduzidos (linhas pontilhadas). As intensidades foram normalizadas em relação aos máximos obtidos com B3LYP para os sistemas completos. Os dados experimentais foram obtidos em solução aquosa em todos os painéis.

A mesma estrutura da banda de A1F obtida utilizando os diferentes funcionais DFT foi observado para os resultados obtidos com INDO/S, tanto na aproximação de excitações simples (ZINDO/S) quanto de excitações duplas (ZINDO/SD). Novamente, uma única excitação para o primeiro estado excitado descreve a banda de A1F na região de baixa energia do espectro. A transição para o segundo estado excitado é proibida por dipolo e, além disso, é $0.4 \mathrm{eV}$ mais energética. Os valores absolutos das energias de transição obtidos com ZINDO/S, considerando somente os compostos isolados, foram equivalentes aos obtidos com os funcionais CAMB3LYP e BHandHLYP. Ao incluir as excitações duplas obtivemos intensidades relativas $8 \%$ menores que as obtidas com excitações simples e as energias do máximo de absorção aumentaram em 0.1 eV. Em relação aos valores experimentais obtidos em solução aquosa, 
vemos diferenças de até $0.3 \mathrm{eV}$. Mais uma vez observamos boa concordância dos resultados teóricos, agora obtidos utilizando método semi-empírico para os compostos isolados, com os resultados experimentais obtidos em solução aquosa e em diclorometano. Também ressaltamos que os efeitos devido à inclusão das excitações duplas não alteraram a descrição do espectro de A1F. Nos cálculos consideramos os seguintes espaços ativos para o composto: 7 completo $\mathrm{OC}=31$ e $\mathrm{NO}=40,7$ reduzido $-\mathrm{OC}=31$ e $\mathrm{NO}=34, \mathbf{1 0}$ completo $-\mathrm{OC}=31$ e $\mathrm{NO}=43$ e 10 reduzido - $\mathrm{OC}=34$ e NO=38. Para as excitações duplas incluímos sempre os orbitais HOMO-1, HOMO, LUMO e LUMO+1. Adotamos $\Gamma=0.6$ eV. Os resultados estão apresentados na Figura 27 e na Tabela 9.

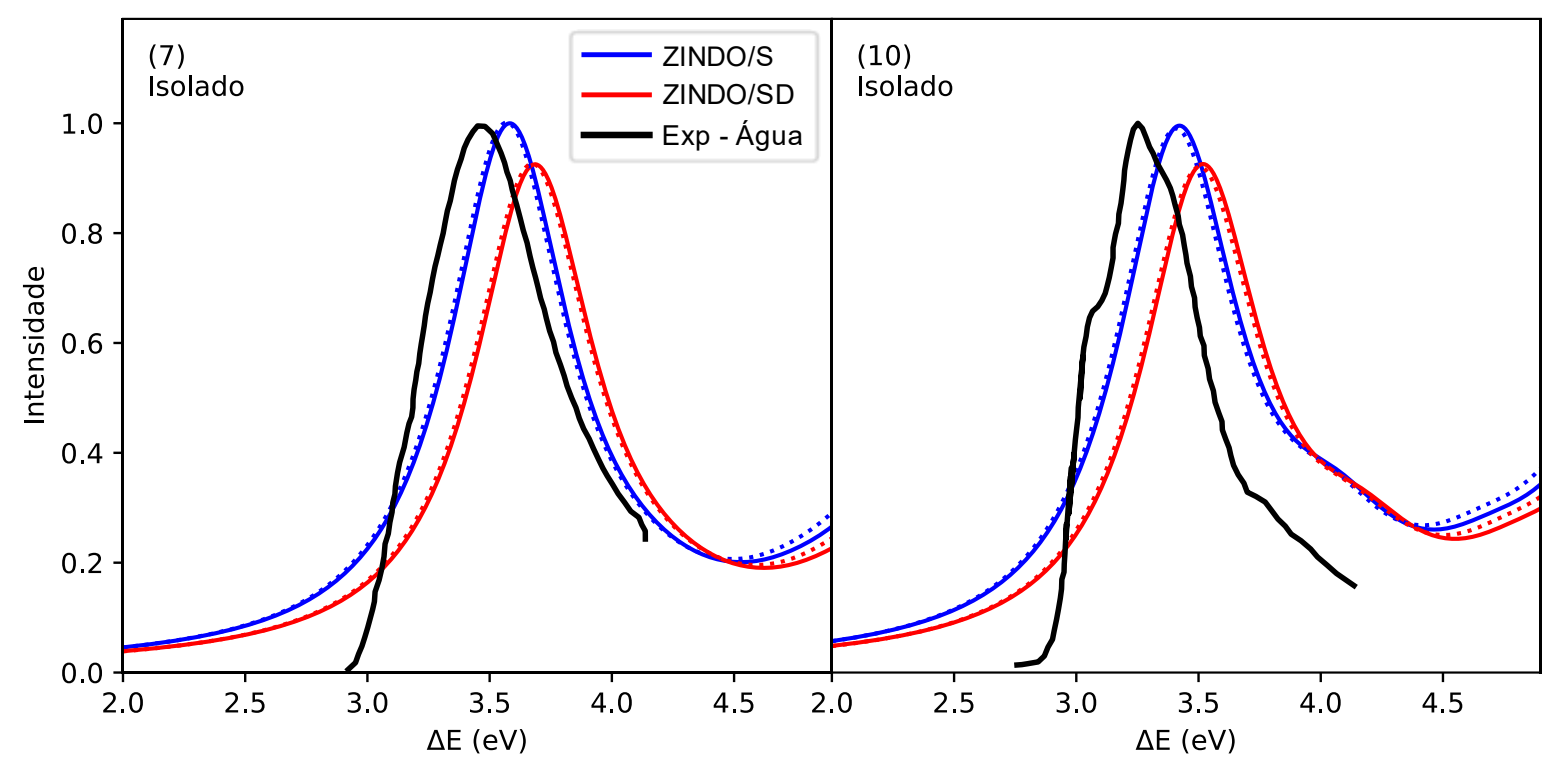

Figura 27. Simulação do espectro de AlF comparando os compostos completo (linhas cheias) e reduzido (linhas pontilhadas). Os espaços ativos selecionados foram: $7-O C=31(31)$ e $N O=40(34) ; 10-O C=31(34)$ e $N O=43(38)$ para compostos completos (reduzidos). Adotamos $\Gamma=0.6 \mathrm{eV}$.

Os resultados das probabilidades de transição de dois fótons foram obtidos para os compostos isolados utilizando diferentes funcionais DFT e utilizando o método INDO/S. Os espectros foram obtidos considerando as 30 transições de menor energia, utilizando DFT; e 200 estados intermediários na soma-sobre-estados, utilizando o método INDO/S. A grande quantidade de átomos, e consecutivamente de elétrons, é fator limitante para a obtenção da A2F utilizando métodos baseados na teoria de resposta de segunda ordem. Além disso, outro fator limitante é a quantidade de pontos necessários para construir a cavidade na aproximação PCM, elevando demasiadamente o custo computacional. Diante dessas dificuldades, as 20 transições eletrônicas de menor energia foram calculadas para os compostos reduzidos com PCM. 
Tabela 9. Valores máximos dos espectros de A1F. Resultados obtidos com conjunto de funções base 6-31+G(d) para cálculos utilizando diferentes funcionais DFT. Os resultados para os compostos reduzidos estão entre parênteses. ${ }^{a}$ Os espaços ativos selecionados foram: $7-O C=31(31)$ e $N O=40(34) ; 10-O C=31(34)$ e $N O=43(38)$ para compostos completos (reduzidos).

\begin{tabular}{|l|c|c|c|}
\hline \multicolumn{1}{|c|}{ Funcional } & Solvente & $\mathbf{7}(\mathrm{eV})$ & $\mathbf{1 0}(\mathrm{eV})$ \\
\hline CAM-B3LYP & Isolado & $3.54(3.55)$ & $3.34(3.36)$ \\
& Água & $3.46(3.46)$ & $3.29(3.30)$ \\
BHandHLYP & Isolado & $3.50(3.51)$ & $3.31(3.32)$ \\
& Água & $3.42(3.42)$ & $3.26(3.26)$ \\
B3LYP & Isolado & $3.05(3.06)$ & $2.90(2.91)$ \\
& Água & $2.97(2.97)$ & $2.84(2.84)$ \\
ZINDO/S & Isolado & $3.58(3.57)$ & $3.42(3.40)$ \\
ZINDO/SD & Isolado & $3.68(3.67)$ & $3.51(3.50)$ \\
EXP & Diclorometano & 3.42 & 3.24 \\
& Água & 3.47 & 3.24 \\
\hline
\end{tabular}

Os resultados obtidos das transições eletrônicas induzidas pela A2F, para todos os métodos aqui avaliados, mostram que a banda de menor energia é descrita por uma única transição envolvendo os orbitais (HOMO-1)-LUMO e HOMO-(LUMO+1) independentemente do valor da energia da transição. Os orbitais obtidos utilizando CAM-B3LYP podem ser vistos na Figura 29. A banda de maior energia é composta por diversas transições eletrônicas, impossibilitando a identificação e caracterização dos orbitais que mais contribuem no espectro. Os resultados obtidos para os sistemas reduzidos mostraram intensidades da ordem de $20 \%$ menores para a banda de baixa intensidade e da ordem de $30-40 \%$ menores para a banda de maior intensidade, comparando com os resultados obtidos para os sistemas completos. Contudo, as energias dos máximos das bandas foram aproximadamente iguais com diferenças menores que $0.04 \mathrm{eV}$. Quando o tensor de transição de dois fótons é obtido utilizando o resíduo da função resposta de segunda ordem, ele contém, de forma indireta, a contribuição de todos os estados excitados intermediários possíveis. Portanto, o fato de o sistema reduzido conter menos orbitais moleculares naturalmente diminui a quantidade de possíveis estados excitados intermediários e impacta diretamente no valor máximo da seção de choque de A2F.

Os resultados obtidos com INDO/S mostram apenas uma banda no espectro de A2F ao considerarmos somente excitações simples (ZINDO/S) e mostram o surgimento da segunda banda ao incluirmos as excitações duplas (ZINDO/SD). Estes resultados evidenciam a importância da inclusão das excitações duplas para descrições mais precisas do espectro de A2F em cálculos utilizando o método INDO/S. As intensidades de A2F relativas entre os compostos completos e reduzidos obtidas com o método soma-sobre-estados mostram diferenças menores que $2 \%$ para a banda de baixa energia e menores que $10 \%$ para a banda de alta energia. Em 
todos os cálculos consideramos 200 estados intermediários garantindo a convergência da propriedade, que será discutida no final desta seção. Apresentamos na Figura 28 os espectros obtidos com $\Gamma=0.4 \mathrm{eV}$, equivalente à largura experimental observada para a banda de alta energia, e na Tabela 10 os valores numéricos para a posição dos máximos das bandas.

Os resultados obtidos com os funcionais DFT com maiores contribuições do termo de troca de Hartree-Fock deslocaram as posições dos máximos dos espectros de A2F em 0.7 eV, para maiores energias, comparativamente aos resultados obtidos com o funcional B3LYP. Acreditamos que esse deslocamento para maiores energias esteja relacionado com uma descrição desbalanceada dos orbitais moleculares devido à maiores contribuições do termo de troca de Hartree-Fock. Lembramos novamente que as intensidades de transição via dois fótons obtidas utilizando o resíduo da função resposta consideram indiretamente a contribuição de todos estados excitados. Portanto, para uma boa descrição das intensidades das transições é necessário boa descrição destes estados e consecutivamente uma boa descrição dos orbitais moleculares. Esse compromisso em descrever os orbitais moleculares de forma balanceada nem sempre é obtido pois, geralmente, a descrição dos orbitais não ocupados de alta energia é negligenciada.

Destacamos a grande dificuldade em descrever os valores da seção de choque de A2F de forma quantitativa para os dois compostos estudados. Enquanto os resultados experimentais para a banda de maior energia são reportados em 275 e 400 GM, respectivamente para os compostos 7 e 10, os resultados teóricos obtidos com PCM atingiram valores acima de 2000 GM para a banda de menor intensidade. Nossos resultados mostram que a banda de menor energia é mais intensa que a banda de maior energia. A razão $\mathrm{X}$ entre as intensidades das bandas obtidas com os funcionais B3LYP, BHandHLYP e CAM-B3LYP são respectivamente 2.6, 2.4 e 3.2 para o composto 7 e 1.6, 1.8 e 2.7 para o composto 10. Outra dificuldade na descrição da A2F utilizando o método TD-DFT refere-se à intensidade relativa entre as bandas. Os resultados sugerem que a banda de menor energia é mais intensa que a de alta energia, mas, geralmente, é esperado que bandas de A2F localizadas em regiões mais energéticas apresentem maiores intensidades. 


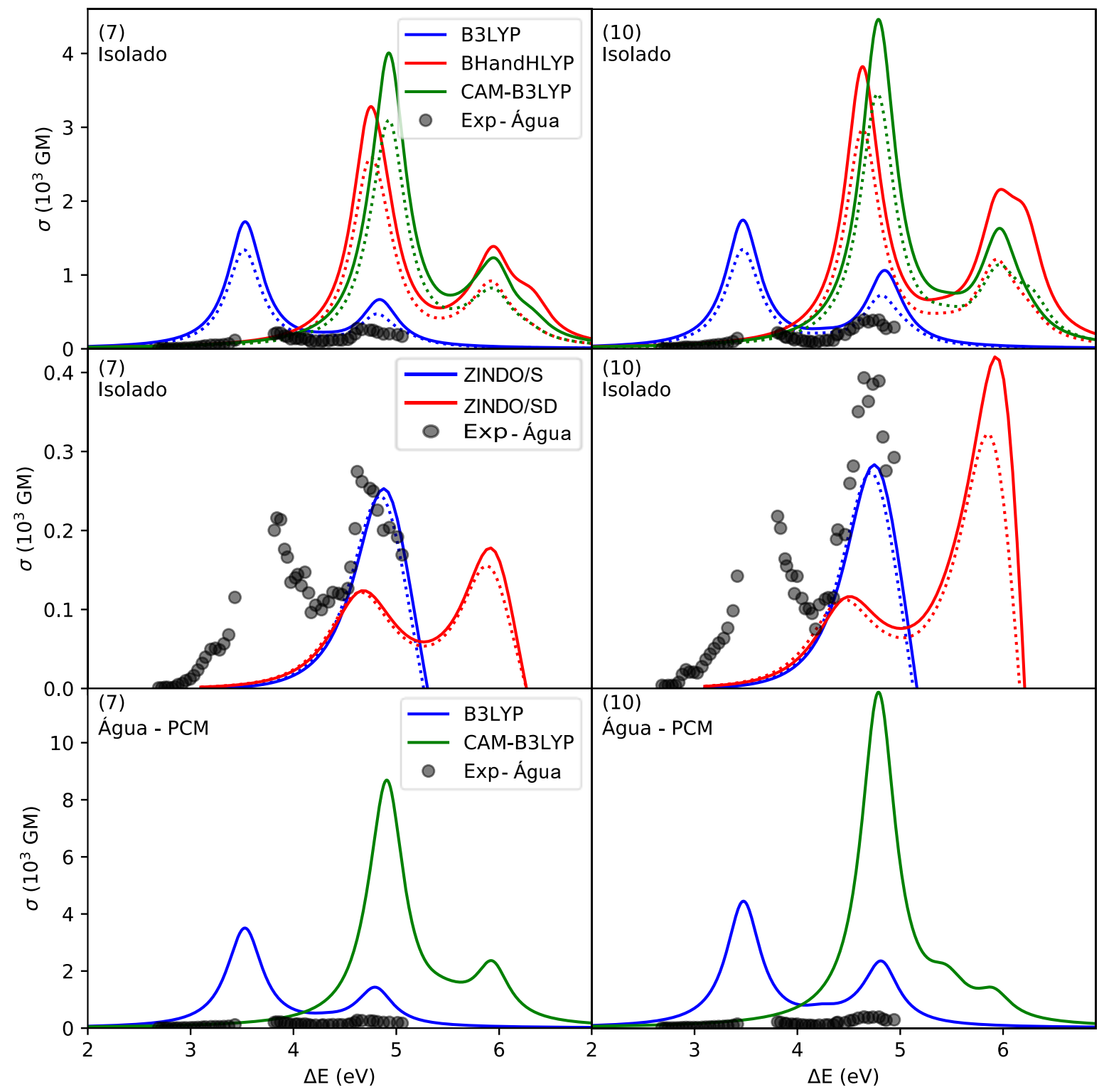

Figura 28. Simulação do espectro de A2F com diferentes funcionais DFT e com o método semi-empírico considerando excitações simples e duplas utilizando a expressão soma-sobre-estados. Os resultados dos compostos completos são apresentados em linhas contínuas e dos compostos reduzidos em linhas pontilhadas, exceto para os últimos quadros, que incluem os efeitos de solventes com PCM, onde as linhas contínuas representam os resultados dos compostos reduzidos. Todos os espectros foram construídos utilizando $\Gamma=0.4 \mathrm{eV}$.

Os resultados obtidos com ZINDO/S mostraram somente uma banda de A2F na região de alta energia e a inclusão das excitações duplas foi essencial para descrever as duas bandas de A2F. Os resultados obtidos com ZINDO/SD mostraram um deslocamento de $1.2 \mathrm{eV}$ na energia do máximo, em relação ao valor experimental, porém, é conhecido que o método INDO/S fornece energias de transições elevadas. Embora a descrição da posição da banda esteja deslocada, os resultados obtidos reproduzem com boa concordância a diferença energética entre bandas de A2F. Diferentemente dos resultados obtidos utilizando TD-DFT, a banda de maior energia obtida com ZINDO/SD resultou em maiores valores da seção de choque de A2F, sendo 1.4 (composto 7) e 3.6 (composto 10) vezes maiores que os valores da banda de baixa energia. 
Além disso, enquanto os resultados obtidos utilizando o método TD-DFT forneceram intensidades extremamente altas (>2000 GM), as intensidades obtidas com ZINDO/SD são 0.65 e 1.05 vezes as experimentais, para os compostos 7 e $\mathbf{1 0}$ respectivamente. Lembramos que os resultados obtidos com ZINDO/S e ZINDO/SD consideram os compostos isolados e que a inclusão dos efeitos de solventes deve melhorar a descrição do espectro de A2F. A implementação do PCM no programa ZINDO utiliza uma única esfera para definir a cavidade do meio contínuo e tal aproximação não é aconselhada para simular as propriedades eletrônicas de compostos alongados, como os avaliados neste trabalho.

Apresentamos na Figura 28 os espectros obtidos atribuindo o valor da largura de linha de $\Gamma=0.4 \mathrm{eV}$ e na Tabela 10 os valores para a posição e intensidade das bandas de baixa $\left(\Delta E_{1}, \sigma_{1}\right)$ e de alta $\left(\Delta E_{2}, \sigma_{2}\right)$ energia.

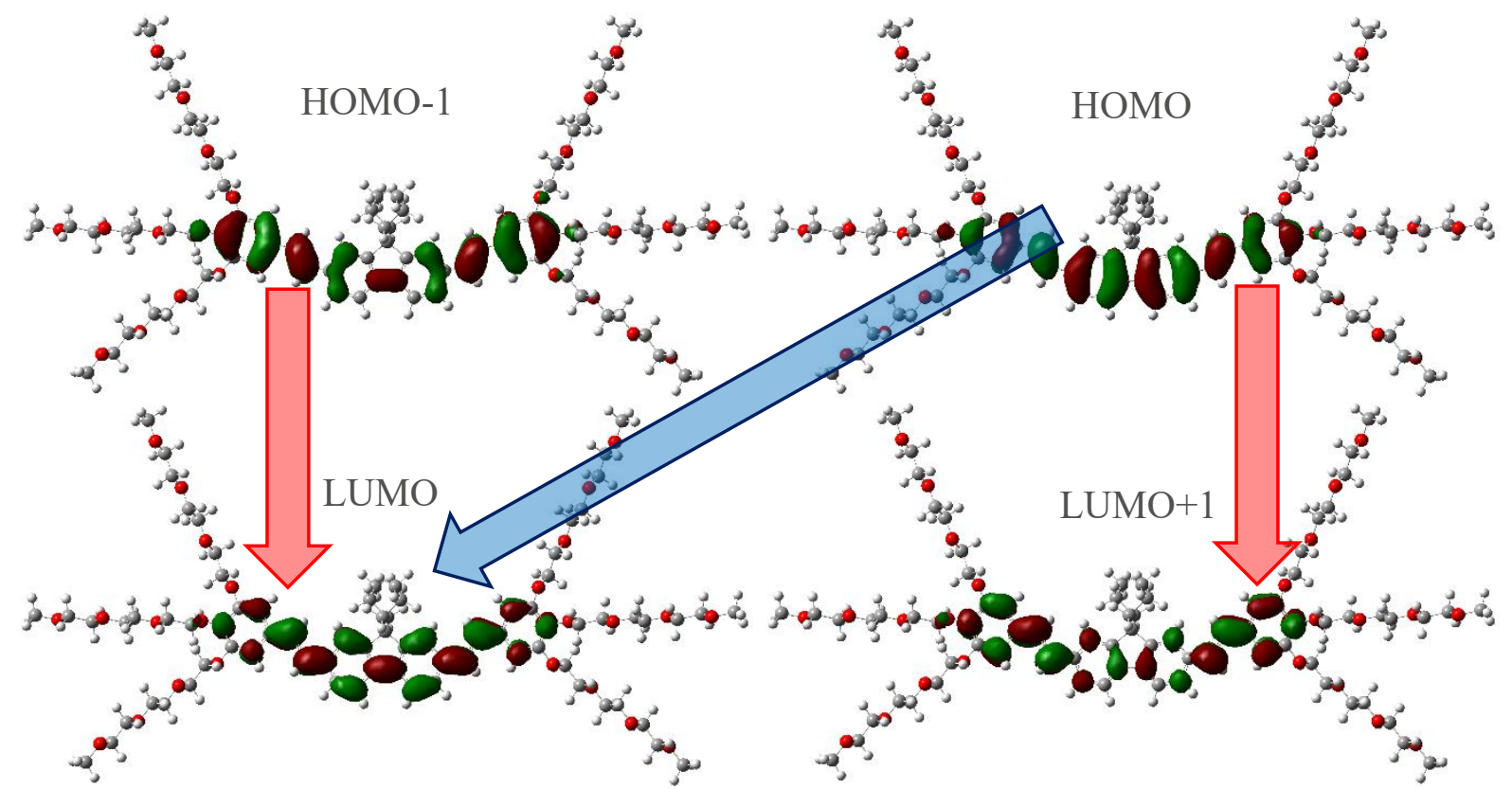

Figura 29. Orbitais moleculares envolvidos nos processos de absorção de um e de dois fótons obtidos com CAM-B3LYP para o composto 10. A seta azul indica o processo de $A 1 F$ e a seta vermelha indica o processo de $A 2 F$. 
Tabela 10. Energia do máximo de absorção da banda de baixa $\left(\Delta E_{1}\right)$ e de alta $\left(\Delta E_{2}\right)$ energia, $\Delta E$ em eV, e intensidade da seção de choque de A2F ( $\sigma-e m G M)$. Resultados dos compostos reduzidos entre parênteses e considerando a aproximação PCM para o solvente água em itálico.

\begin{tabular}{|lcccc|}
\hline $\mathbf{7}$ & $\Delta \mathrm{E}_{1}$ & $\sigma_{1}$ & $\Delta \mathrm{E}_{2}$ & $\sigma_{2}$ \\
\hline B3LYP & 3.54 & 1722 & 4.84 & 668 \\
& $(3.52)$ & $(1345)$ & $(4.82)$ & $(503)$ \\
BHandHLYP & $(3.54)$ & $(3507)$ & $(4.80)$ & $(1436)$ \\
& 4.76 & 3280 & 5.96 & 1388 \\
CAM-B3LYP & $(4.76)$ & $(2587)$ & $(5.96)$ & $(919)$ \\
& 4.92 & 4007 & 5.96 & 1234 \\
& $(4.92)$ & $(3095)$ & $(5.94)$ & $(823)$ \\
ZINDO/S & $(4.92)$ & $(8690)$ & $(5.92)$ & $(2367)$ \\
& 4.88 & 253 & - & - \\
ZINDO/SD & $(4.84)$ & $(243)$ & & \\
& 4.66 & 124 & 5.92 & 178 \\
EXP & $(2.33)$ & $(122)$ & $(5.88)$ & $(156)$ \\
\hline 10 & - & - & 4.76 & 275 \\
\hline B3LYP & $\Delta \mathrm{E}_{1}$ & $\sigma_{1}$ & $\Delta \mathrm{E}_{2}$ & $\sigma_{2}$ \\
& 3.48 & 1744 & 4.86 & 1062 \\
& $(3.48)$ & $(1346)$ & $(4.82)$ & $(725)$ \\
BHandHLYP & $(3.48)$ & $(4449)$ & $(4.82)$ & $(2356)$ \\
& 4.64 & 3821 & 5.98 & 2159 \\
CAM-B3LYP & $(4.64)$ & $(2967)$ & $(5.94)$ & $(1211)$ \\
& 4.78 & 4462 & 5.96 & 1632 \\
ZINDO/S & $(4.78)$ & $(3453)$ & $(5.98)$ & $(1141)$ \\
& $(4.80)$ & $(11770)$ & $(5.88)$ & $(1440)$ \\
ZINDO/SD & 4.74 & 283 & - & - \\
EXP & $(4.70)$ & $(273)$ & & \\
& 4.54 & 116 & 5.92 & 420 \\
& $(4.44)$ & $(113)$ & $(5.84)$ & $(321)$ \\
& - & - & 4.68 & 400 \\
\hline
\end{tabular}

Na Figura 30 apresentamos a variação do resultado obtido com o método soma-sobreestados considerando 50, 100, 150 e 200 estados intermediários para o composto 10. A convergência é mais lenta quando consideramos as excitações duplas, embora os resultados com 100 estados intermediários estejam suficientemente convergidos. 


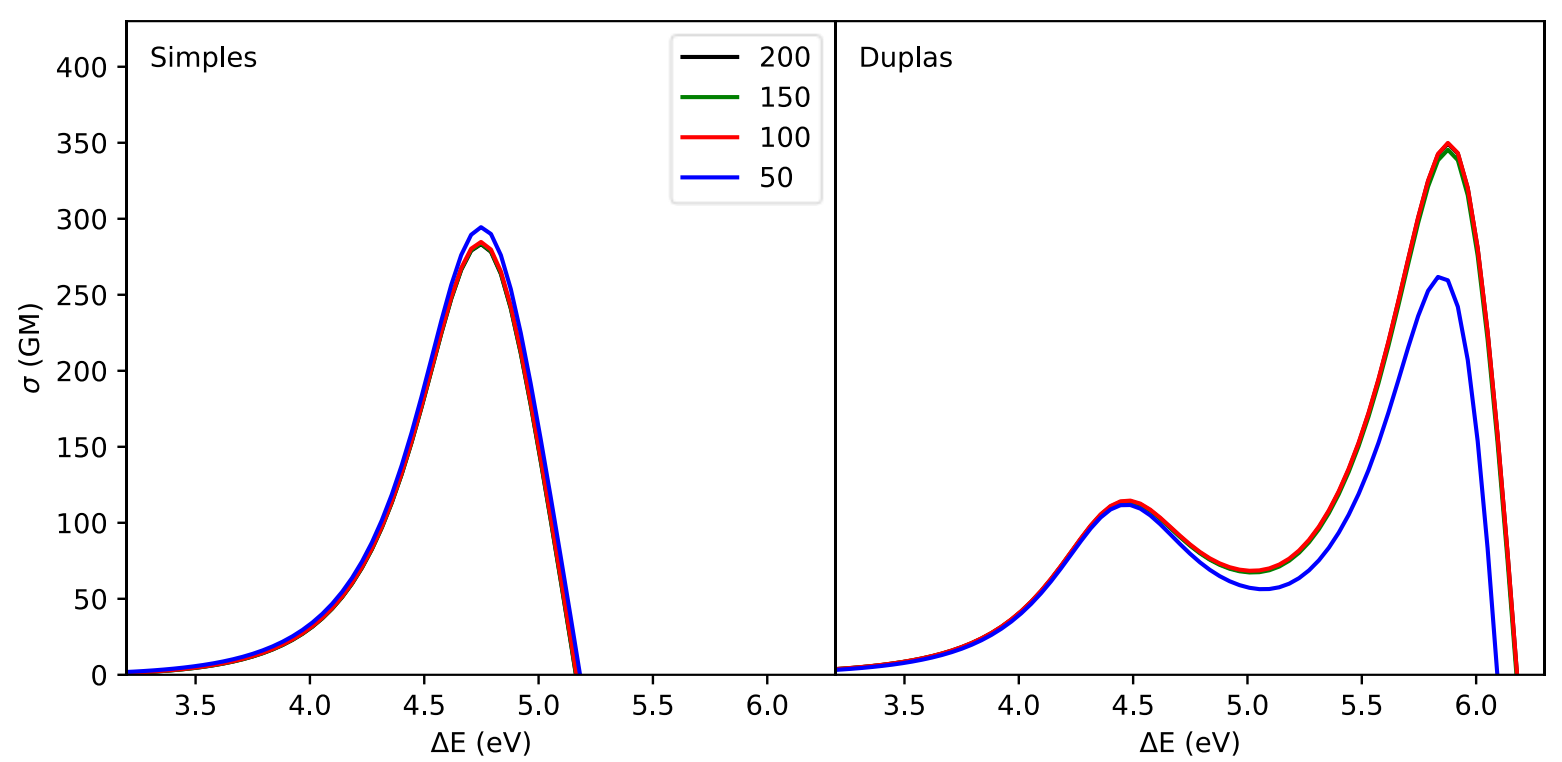

Figura 30. Variação do resultado obtido com o método soma-sobre-estados considerando 50, 100, 150 e 200 estados intermediários para o composto 10. Considerando apenas excitações simples (esquerda) e excitações simples e duplas (direita).

\subsubsection{Conclusões}

Neste trabalho, as intensidades da seção de choque de A2F, as transições eletrônicas e a influência das cadeias hidrofílicas na composição das bandas de A2F foram estudadas teoricamente para complementar a descrição de um estudo experimental disponível na literatura. Os resultados foram obtidos utilizando a teoria de resposta quadrática com DFT e calculando explicitamente a soma-sobre-estados utilizando o método INDO/S. Os efeitos de solventes foram incluídos utilizando a aproximação PCM. Ao desconsiderarmos as cadeias hidrofílicas obtivemos a mesma transição eletrônica, confirmando o papel destas cadeias em aumentar a solubilidade sem alterar a intensidade de A2F.

Obtivemos boa descrição do espectro de A1F utilizando funcionais DFT com maiores contribuições do termo de troca exato de HF, como nos funcionais CAM-B3LYP e BHandHLYP. Por outro lado, o resultado obtido com o funcional B3LYP descreve a posição as bandas de $\mathrm{A} 2 \mathrm{~F}$ em melhor concordância com os resultados experimentais. A banda de A2F de baixa energia, não descrita experimentalmente, envolve transições entre os orbitais (HOMO1)-LUMO e HOMO-(LUMO+1). De forma geral, os resultados obtidos para a seção de choque de A2F com TD-DFT foram extremamente altos, principalmente quando consideradas as interações com o solvente na aproximação PCM.

Os espectros de A2F obtidos utilizando o método INDO/S descrevem os valores máximos da seção de choque de $\mathrm{A} 2 \mathrm{~F}$ e a intensidade relativa entre as bandas de baixa e alta energia. A inclusão das excitações duplas nos cálculos foi essencial para obter tais resultados. 
Além disso, o método INDO/S apresenta outra grande vantagem: o baixo custo computacional. Portanto, a utilização do método INDO/S com excitações simples e duplas é uma excelente alternativa para estudar a seção de choque de A2F de grandes moléculas.

\subsection{Desvendando a resposta EFISHG de complexos de stilbazolium formados por pares iônicos em solução utilizando método de simulação multiescala}

\subsubsection{Introdução}

A técnica experimental EFISHG é conhecida por determinar a primeira $(\boldsymbol{\beta})$ e segunda ( $\boldsymbol{\gamma}$ ) hiperpolarizabilidades de compostos em solução. Para realizar as medidas, campos eletrostáticos são aplicados na amostra para quebrar a isotropicidade e criar uma orientação preferencial. Consequentemente, a técnica EFISHG não pode ser utilizada para investigar compostos carregados devido principalmente à avaliação das componentes de $\beta$ projetadas sobre as componentes do momento de dipolo molecular [27]. Contudo, além de muitas aplicações em moléculas neutras, recentemente a técnica EFISHG foi utilizada para determinar as respostas ONL de complexos neutros constituídos por pares ânion-cátion. Para investigar essa categoria de compostos neutros é necessário utilizar solventes de baixa polaridade, como $\mathrm{CHCl}_{3}$, para favorecer a associação do par e evitar a indesejada dissociação. Isso foi demonstrado pela primeira vez no ano de 2000 para cromóforos anfifílicos [28] e mais recente para chaves moleculares acionadas por variações de pH [29]. Embora existam medidas da resposta EFISHG para complexos neutros, poucos estudos teóricos foram realizados nessa categoria de complexos em solução. Por consequência, os efeitos das espécies carregadas na resposta EFISHG são pouco conhecidos. A dificuldade associada com a inclusão dos efeitos térmicos e ambientais nas geometrias dos complexos e nas posições relativas entre o par ânioncátion são alguns dos motivos para o reduzido número de estudos teóricos já realizados.

Neste trabalho, a relação entre propriedades geométricas e as respostas EFISGH foram avaliadas para cátions de stilbazolium e ânions de diferentes tamanhos (desde átomos inorgânicos como o iodeto até a molécula orgânica $p$-toluenosulfonato). Os cátions de stilbazolium apresentam grande resposta ONL de segunda ordem devido a sua estrutura de conjugação $\pi$. As estruturas químicas dos complexos estão apresentadas na Figura 31. A metodologia multiescala S-QM/MM foi utilizada para: incluir a distribuição do ânion ao redor do cátion e os efeitos térmicos utilizando dinâmicas moleculares clássicas (DM); e para obter as respostas ONL médias. Além disso, quantificamos a correlação estatística entre a estrutura 
molecular e as respostas ONL. No primeiro passo, DM foram realizadas para gerar configurações representativas dos complexos em clorofórmio, e em seguida, no segundo passo, métodos de mecânica quântica (QM) foram utilizados para obter as propriedades ONL nestas configurações. Além disso, análises estatísticas foram realizadas para quantificar as relações entre as propriedades geométricas e eletrônicas. Para as DM utilizamos o campo de força OPLS-AA reparametrizado para reproduzir a geometria de mínima energia obtida utilizando o método DFT. As propriedades ópticas lineares e não lineares foram obtidas para esse conjunto de configurações utilizando os métodos TD-DFT e Coupled Perturbed Kohn-Sham (CPKS). Como será demonstrado, a posição relativa entre o cátion e o ânion são importantes para a descrição da resposta EFISHG devido à habilidade de controlar o momento de dipolo permanente. Por outro lado, as componentes do tensor da primeira hiperpolarizabilidade estão fracamente correlacionadas com o comprimento de conjugação $\pi$ do cátion.

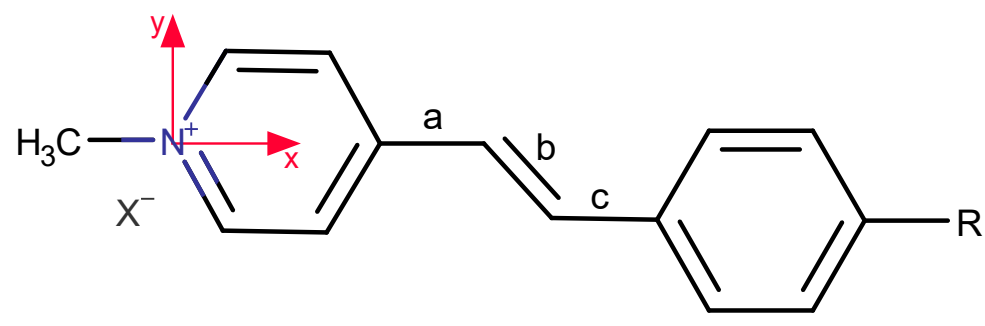

$\begin{array}{lllll}\text { Complexo: } & \mathbf{1}-\mathrm{R}=\mathrm{H} & \mathrm{X}=\mathrm{I} & \mathbf{4 . 1}-\mathrm{R}=\mathrm{N}(\mathrm{n}-\mathrm{Bu})_{2} & \mathrm{X}=\mathrm{CF}_{3} \mathrm{SO}_{3} \\ \mathbf{2}-\mathrm{R}=\text { tert }-\mathrm{Bu} & \mathrm{X}=\mathrm{I} & \mathbf{4 . 2}-\mathrm{R}=\mathrm{N}(\mathrm{n}-\mathrm{Bu})_{2} & \mathrm{X}=\mathrm{ClO}_{4} \\ \mathbf{3}-\mathrm{R}=\mathrm{N}(\mathrm{Me})_{2} & \mathrm{X}=\mathrm{I} & \mathbf{4 . 3}-\mathrm{R}=\mathrm{N}(\mathrm{n}-\mathrm{Bu})_{2} & \mathrm{X}=\mathrm{SCN} \\ \mathbf{4}-\mathrm{R}=\mathrm{N}(\mathrm{n}-\mathrm{Bu})_{2} & \mathrm{X}=\mathrm{I} & \mathbf{4 . 4}-\mathrm{R}=\mathrm{N}(\mathrm{n}-\mathrm{Bu})_{2} & \mathrm{X}=\mathrm{pTS} *\end{array}$

Figura 31. Estrutura do cátion stilbazolium e lista dos ânions. *p-toluenosulfonato.

\subsubsection{Detalhes computacionais}

As DM foram realizadas em uma caixa cúbica periódica adotando o ensemble NPT em condições ambientes $(\mathrm{T}=298.15 \mathrm{~K}$ e $\mathrm{p}=1 \mathrm{~atm})$. As caixas iniciais, contendo um par de íons e 1500 moléculas de clorofórmio, foram previamente termalizadas utilizando o método de $\mathrm{MC}$, com moléculas rígidas, utilizando o programa DICE [97]. Pra cada complexo, a configuração após $2.25 \times 10^{8}$ passos de MC foi utilizada como condição inicial para DM considerando as geometrias flexíveis no programa Gromacs [164,165]. Para avaliar os efeitos do ânion ao redor do cátion, $4.5 \times 10^{8}$ passos de MC foram realizados adicionalmente considerando as geometrias rígidas. As equações das DM, sem restrições geométricas, foram propagadas em passos de $1 \mathrm{fs}$ utilizando o algoritmo leap-frog com duração total de $30 \mathrm{~ns}$ e de 50 ns, respectivamente para os complexos 1-4 e 4.1-4.4. Termalizações adicionais de $5 \mathrm{~ns}$ foram consideradas, portanto, 
selecionamos as configurações dos 25 ns e 45 ns finais, respectivamente para os complexos 14 e 4.1-4.4. Para manter a temperatura constante acoplamos o termostato de escalonamento das velocidades [166] a cada 100 passos de integração e para manter a pressão constante utilizamos o barostato de Parrinello-Rahman [167] a cada 1000 passos. O raio de corte foi definido em 14.0 ̊̊ para interações não ligadas de curto alcance enquanto as correções eletrostáticas de longo alcance foram computadas utilizando o método smooth Particle-Mesh Ewald [168]. O campo de força OPLS-AA proposto por McDonald [169] foi utilizado para o solvente clorofórmio.

As geometrias iniciais para os pares iônicos utilizados nas DM foram obtidas com cálculos de minimização de energia implementado no programa Gaussian 16 [170] utilizando PCM/ $\omega$ B97X-D/aug-cc-pVDZ. O conjunto de funções base aug-cc-pVDZ com pseudo potencial (identificada por aug-cc-pVDZ-PP) foi utilizado para o átomo de iodo em todos os cálculos. O funcional DFT $\omega$ B97X-D [82,83], o qual inclui correções de longo alcance para o termo de troca de HF e correções empíricas de London para as contribuições de dispersão, foi escolhido por apresentar resultados similares aos obtidos utilizando MP2 para os complexos 13. Os parâmetros de equilíbrio do campo de força foram definidos com as distâncias obtidas nas geometrias de mínima energia, enquanto as cargas dos sítios atômicos foram definidas utilizando o ajuste do potencial eletrostático CHELPG em nível de cálculo PCM/M06-2X/augcc-pVDZ. Para cada trajetória, extraímos das simulações moleculares configurações a cada 125 ps e 225 ps para os complexos com e sem o iodeto, respectivamente, totalizando 200 configurações estatisticamente descorrelacionadas para cada simulação. Para a simulação adicional com geometrias rígidas as configurações foram selecionadas a cada $2.25 \times 10^{6}$ passos de MC.

As componentes do tensor $\beta$ e $\gamma$ foram calculadas com PCM/M06-2X/6-311+G(d) utilizando o método TD-DFT para 200 configurações de cada simulação. Definimos o valor de campo elétrico dinâmico de $1907 \mathrm{~nm}$, o qual foi o utilizado nos experimentos. As componentes dos tensores ONLs foram combinados para obter $\beta_{/ /}, \gamma_{/ /}$e $\beta_{H R S}$ como descrito na seção 2.2.4 e, finalmente, calculamos os valores médios das propriedades ONLs. Destas configurações, somente a estrutura molecular dos complexos foram extraídas, portanto, não incluímos moléculas de clorofórmio nos cálculos das propriedades ONL. As interações soluto-solvente foram incluídas com PCM e todos os cálculos foram realizados no programa Gaussian 16 [170]. 


\subsubsection{Discussões}

Todas as geometrias do estado fundamental utilizadas para parametrizar o campo de força OPLS-AA foram obtidas empregando o funcional híbrido $\omega$ B97X-D e o conjunto de funções bases aug-cc-pVDZ(-PP). As condições de mínima energia foram confirmadas pelo sinal positivo das derivadas de segunda ordem da energia. Em todos os cálculos QM, os efeitos do solvente clorofórmio foram aproximados com PCM. A validade das geometrias do estado fundamental com $\omega$ B97X-D foi aferida para os complexos 1-3 por comparação com as geometrias obtidas com MP2. Além das características geométricas, cargas atômicas e respostas ONL também foram comparadas e mostram ótima concordância (ver Tabela 11 eTabela 12). A excelente concordância observada entre os dois conjuntos de geometrias, garante uma descrição confiável da estrutura de mínima energia dos complexos com um menor custo computacional. O caráter iônico dos complexos foi confirmado pelas cargas atômicas calculadas empregando o ajuste eletrostático CHELPG com PCM/M06-2X/aug-cc-pVDZ. De fato, os valores absolutos para a carga de ânions/cátions (dados pelas somas das cargas atômicas) atingem 0.91 para os complexos 1-4 e 0.92, 0.94, 0.95 e 0.95 para os complexos 4.1, 4.2, $\mathbf{4 . 3}$ e 4.4, respectivamente.

Tabela 11. Valores EFISHG e HRS obtidos para perturbações dinâmicas com $1907 \mathrm{~nm}$ em função do método de otimização de geometria e da posição do ânion. Os valores reportados estão nas unidades $\mu \beta_{/ /}\left(10^{3}\right.$ a.u.), $\beta_{/ /}($a.u. $), \mu($ a.u. $), \theta_{(\mu, \beta)}($ deg $)$ é o ângulo entre os vetores $\mu$ e $\beta, \beta_{H R S}$ (a.u.).

\begin{tabular}{|c|c|c|c|c|c|c|c|c|}
\hline Complexo & $\begin{array}{l}\text { Otimização } \\
\text { de geometria }\end{array}$ & $\begin{array}{l}\text { Posição do } \\
\text { ânion }\end{array}$ & $\mu \beta_{/ /}$ & $\beta_{/ /}$ & $\mu$ & $\theta_{(\mu, \beta)}$ & $\beta_{H R S}$ & DR \\
\hline \multirow{4}{*}{1} & MP2 & No plano & 25.00 & 2954 & 8.46 & 58.8 & 4080 & 4.48 \\
\hline & MP2 & Fora do plano & 4.71 & 769 & 6.13 & 81.1 & 3629 & 4.26 \\
\hline & $\omega B 97 X-D$ & No plano & 23.48 & 2659 & 8.83 & 60.1 & 3822 & 4.46 \\
\hline & $\omega B 97 X-D$ & Fora do plano & 4.93 & 750 & 6.57 & 81.0 & 3485 & 4.31 \\
\hline \multirow{4}{*}{2} & MP2 & No plano & 57.54 & 6270 & 9.18 & 44.6 & 6232 & 4.64 \\
\hline & MP2 & Fora do plano & 13.42 & 2151 & 6.24 & 74.1 & 5615 & 4.51 \\
\hline & $\omega B 97 X-D$ & No plano & 54.72 & 5744 & 9.53 & 45.5 & 5796 & 4.63 \\
\hline & $\omega B 97 X-D$ & Fora do plano & 14.45 & 2141 & 6.75 & 73.4 & 5354 & 4.51 \\
\hline \multirow{4}{*}{3} & MP2 & No plano & 196.69 & 19071 & 10.31 & 37.1 & 16746 & 4.78 \\
\hline & MP2 & Fora do plano & 82.76 & 11392 & 7.27 & 58.0 & 15108 & 4.72 \\
\hline & $\omega B 97 X-D$ & No plano & 235.21 & 21326 & 11.03 & 36.5 & 18586 & 4.78 \\
\hline & $\omega B 97 X-D$ & Fora do plano & 110.30 & 14194 & 7.77 & 54.9 & 17320 & 4.76 \\
\hline \multirow{2}{*}{4} & $\omega B 97 X-D$ & No plano & 242.48 & 22975 & 10.55 & 44.5 & 22477 & 4.82 \\
\hline & $\omega B 97 X-D$ & Fora do plano & 135.20 & 17348 & 7.79 & 54.4 & 20867 & 4.80 \\
\hline 4.1 & $\omega B 97 X-D$ & Fora do plano & 155.23 & 20424 & 7.60 & 45.4 & 20259 & 4.86 \\
\hline 4.2 & $\omega B 97 X-D$ & Fora do plano & 135.47 & 18054 & 7.50 & 51.7 & 20305 & 4.86 \\
\hline 4.3 & $\omega B 97 X-D$ & Fora do plano & 94.25 & 13996 & 6.73 & 60.3 & 19770 & 4.78 \\
\hline 4.4 & $\omega B 97 X-D$ & Fora do plano & 114.97 & 20308 & 5.66 & 41.1 & 18804 & 4.83 \\
\hline
\end{tabular}


Tárcius N. Ramos

Tabela 12. Valores das respostas EFISHG para perturbações dinâmicas com 1907 nm em função do método de otimização de geometria e da posição do ânion. Os valores reportados estão na unidades: $\mu \beta_{/ /} / k T$, $\gamma_{/ /}$e $\gamma_{E F I S H G}\left(10^{4}\right.$ au $) ;\left[\mu \beta_{/ /}\right]_{e f f} e$ $\left[\mu \beta_{/ /}\right]_{\text {exp }}\left(10^{-48}\right.$ esu $), R_{3 / 2}=\left(3 k T \times \gamma_{/ /}\right) / \mu \beta_{/ /} . T=298.15 \mathrm{~K}, 3 k T=2.833 \times 10^{-3}$ u.a.

\begin{tabular}{|c|c|c|c|c|c|c|c|}
\hline Complexo & $\begin{array}{l}\text { Otimização } \\
\text { de geometria }\end{array}$ & $\begin{array}{l}\text { Posição do } \\
\text { ânion }\end{array}$ & $\mu \beta_{/ /} / 3 k T$ & $\gamma_{/ /}$ & $\gamma_{E F I S H G}$ & $R_{3 / 2}$ & {$\left[\mu \beta_{/ /}\right]_{e f f}$} \\
\hline \multirow{4}{*}{1} & MP2 & No plano & 883 & 52.1 & 935 & 0.06 & 26.5 \\
\hline & MP2 & Fora do plano & 166 & 56.7 & 223 & 0.34 & 6.3 \\
\hline & $\omega B 97 X-D$ & No plano & 829 & 48.3 & 877 & 0.06 & 24.8 \\
\hline & $\omega B 97 X-D$ & Fora do plano & 174 & 52.0 & 226 & 0.30 & 6.4 \\
\hline \multirow{4}{*}{2} & MP2 & No plano & 2031 & 82.2 & 2114 & 0.04 & 59.9 \\
\hline & MP2 & Fora do plano & 474 & 82.0 & 556 & 0.17 & 15.7 \\
\hline & $\omega B 97 X-D$ & No plano & 1931 & 75.6 & 2007 & 0.04 & 56.9 \\
\hline & $\omega B 97 X-D$ & Fora do plano & 510 & 75.7 & 586 & 0.15 & 16.6 \\
\hline \multirow{4}{*}{3} & MP2 & No plano & 6944 & 200.1 & 7144 & 0.03 & 202.4 \\
\hline & MP2 & Fora do plano & 2922 & 189.6 & 3111 & 0.06 & 88.1 \\
\hline & $\omega \mathrm{B} 97 \mathrm{X}-\mathrm{D}$ & No plano & 8304 & 208.8 & 8513 & 0.03 & 241.1 \\
\hline & $\omega B 97 X-D$ & Fora do plano & 3894 & 200.5 & 4094 & 0.05 & 116.0 \\
\hline \multirow{2}{*}{4} & $\omega B 97 X-D$ & No plano & 8560 & 256.7 & 8817 & 0.03 & 249.7 \\
\hline & $\omega B 97 X-D$ & Fora do plano & 4773 & 248.7 & 5022 & 0.05 & 142.2 \\
\hline 4.1 & $\omega B 97 X-D$ & Fora do plano & 5480 & 227.5 & 5708 & 0.04 & 161.7 \\
\hline 4.2 & $\omega B 97 X-D$ & Fora do plano & 4783 & 230.1 & 5013 & 0.05 & 142.0 \\
\hline 4.3 & $\omega B 97 X-D$ & Fora do plano & 3327 & 228.4 & 3556 & 0.07 & 100.7 \\
\hline 4.4 & $\omega \mathrm{B} 97 \mathrm{X}-\mathrm{D}$ & Fora do plano & 4059 & 221.5 & 4280 & 0.05 & 121.2 \\
\hline
\end{tabular}

Uma boa descrição da alternância do comprimento das ligações (Bond Length Alternation $-\mathrm{BLA}=(a+c) / 2-b$, definida pelas ligações $a, b$, e $c$ na Figura 31) é requerida para obter valores confiáveis para as respostas ópticas lineares e não lineares pois os elétrons $\pi$ conjugados exercem importante papel na descrição de efeitos de campos não locais. Este é o caso para os compostos com caráter push-pull e para pares iônicos. Além da descrição do BLA, a distância entre o cátion e o ânion ( $\left.\mathrm{d}_{\mathrm{NA}}\right)$ foi considerada nas análises. Definida como a distância entre o nitrogênio "positivo" do cátion com o átomo A do ânion, onde A é o átomo I, S, Cl, C e S respectivamente para os ânions $\mathrm{I}^{-}, \mathrm{CF}_{3} \mathrm{SO}_{3}{ }^{-}, \mathrm{ClO}_{4}^{-}, \mathrm{SCN}^{-}$e pTS . Esta distância cátion-ânion é um parâmetro importante para avaliar possíveis correlações com as propriedades eletrônicas. Os valores da BLA e $\mathrm{d}_{\mathrm{NA}}$ obtidos nas DM estão apresentados na Tabela 13, na mesma tabela estão os valores da BLA obtidos para as geometrias DFT de mínima energia.

Os valores da BLA são pouco menores ( 0.02 $⿱$ A) para os complexos $\mathbf{3}$ e 4 comparativamente com os valores obtidos para os complexos 1 e 2. Atribuímos essa diferença à presença do grupo doador dialkylamino. A diferença entre os valores médios da BLA com os valores obtidos para as geometrias DFT de mínima energia são inferiores a $0.003 \AA$ 
comprovando uma boa parametrização do campo de força. Os efeitos dos diferentes ânions nos valores da BLA são menores que $0.01 \AA$ A. Além disso, a mesma tendência foi observada para as geometrias de mínima energia obtidas com $\omega$ B97X-D.

Tabela 13. Valores médios e desvio padrão da BLA $(\AA)$ e da distância $d_{N A}(\AA)$ obtidos das DM. Os valores da BLA das geometrias DFT de mínima energia obtidas estão entre parênteses.

\begin{tabular}{|c|c|c|}
\hline Complexo & BLA & $\mathrm{d}_{\mathrm{NA}}$ \\
\hline $1^{*}$ & - & $4.340 \pm 0.491$ \\
\hline 1 & $0.114 \pm 0.029(0.117)$ & $4.289 \pm 0.412$ \\
\hline 2 & $0.117 \pm 0.031(0.115)$ & $4.280 \pm 0.415$ \\
\hline 3 & $0.099 \pm 0.031(0.098)$ & $4.337 \pm 0.366$ \\
\hline 4 & $0.092 \pm 0.028(0.095)$ & $4.450 \pm 0.496$ \\
\hline 4.1 & $0.095 \pm 0.028(0.094)$ & $4.390 \pm 0.440$ \\
\hline 4.2 & $0.096 \pm 0.030(0.098)$ & $4.400 \pm 0.522$ \\
\hline 4.3 & $0.097 \pm 0.029(0.098)$ & $4.184 \pm 0.535$ \\
\hline 4.4 & $0.102 \pm 0.031(0.101)$ & $4.273 \pm 0.338$ \\
\hline
\end{tabular}

* Cálculos realizados para a simulação com geometrias rígidas.

Durante as DMs a ligação iônica manteve o ânion em volta do cátion como pode ser observado na Figura 32 onde sobrepomos as 200 configurações (somente uma configuração foi utilizada para ilustrar a posição do cátion). Os valores de $\mathrm{d}_{\mathrm{NA}}, \mathrm{A}=\mathrm{I}$, obtidos para os complexos 1-4 quando o iodeto está no mesmo plano que o anel são de 4.5 Å e quando o iodeto está no mesmo plano que o anel do cátion e de 3.7 Å quando o ânion está sobre o anel. A dependência dos valores médios de $\mathrm{d}_{\mathrm{NA}}$ em função dos diferentes ânions é pequena comparada com os valores dos desvios padrão.

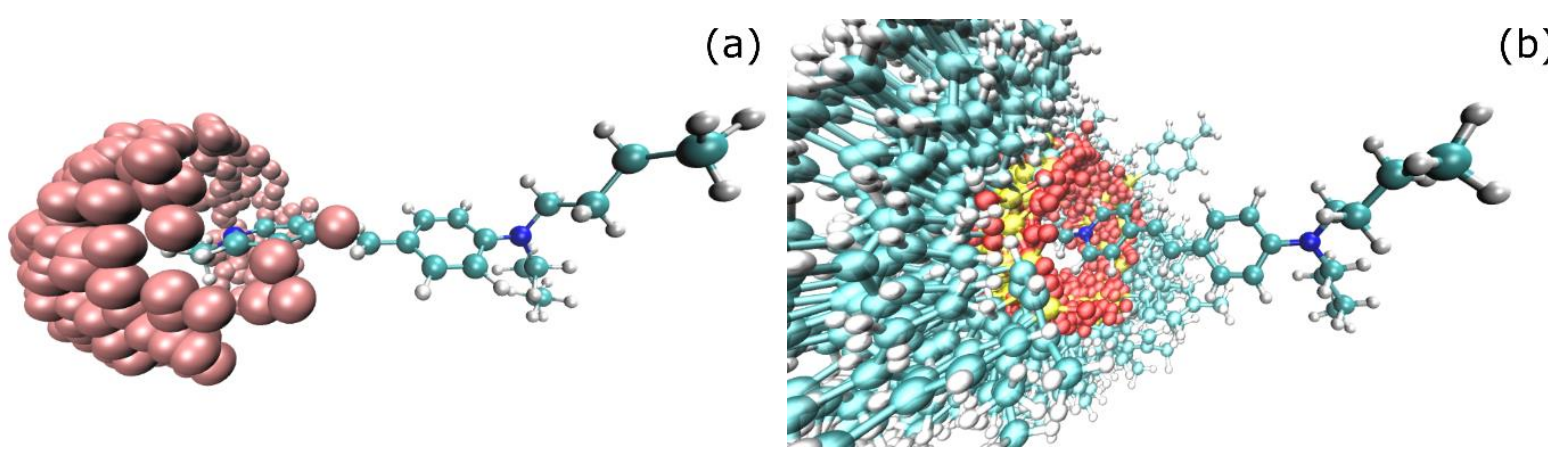

Figura 32.(a) Posição relativa do I' e (b) do pTS ao redor do cátion do complexo 4. As geometrias dos cátions representam única configuração enquanto as distribuições dos ânions representam a sobreposição de 200 configurações.

As transições para os cinco estados excitados de menor energia das 200 configurações foram consideradas para construir os espectros de A1F de cada complexo. Para cada energia de transição e força do oscilador fora associada uma função Lorentziana, para representar o alargamento espectral considerando o valor de $\Gamma=0.4 \mathrm{eV}$ para a largura completa a meia altura. 
Os espectros foram normalizados em referência ao valor máximo de 1.38 obtido para o complexo 1. O comprimento de onda associado com o valor máximo de absorção deslocou de $349 \mathrm{~nm}$ até $445 \mathrm{~nm}$ respectivamente para os complexos 1-4 enquanto a intensidade relativa aumentou em 21\% (ver Tabela 14). As excitações intensas correspondem a transições de transferências de carga do grupo doador fenil ou do anel amino-fenil para o grupo aceitador piridínio (ver Figura 33).

Tabela 14. Comprimentos de onda (nm) associados com os máximos das absorções. As intensidades teóricas estão normalizadas em referência ao complexo 1 que apresenta o valor de 1.38 para a intensidade máxima.

\begin{tabular}{|c|cccc|}
\hline Complexo & $\boldsymbol{\lambda}$ & Intensidade & $\boldsymbol{\lambda}_{\text {exp }}{ }^{\mathbf{a}}$ \\
\hline $\mathbf{1}^{*}$ & 335 & 1.28 & 357 \\
$\mathbf{1}$ & 349 & 1.00 & 357 \\
$\mathbf{2}$ & 356 & 1.08 & 377 \\
$\mathbf{3}$ & 438 & 1.14 & 503 \\
$\mathbf{4}$ & 445 & 1.21 & 519 \\
$\mathbf{4 . 1}$ & 439 & 1.23 & 515 \\
$\mathbf{4 . 2}$ & 438 & 1.23 & 520 \\
$\mathbf{4 . 3}$ & 441 & 1.19 & 515 \\
$\mathbf{4 . 4}$ & 431 & 1.20 & 509 \\
\hline
\end{tabular}

Em todos os casos as transições intensas são do tipo $\pi \rightarrow \pi^{*}$ para o orbital LUMO. Para os complexos 1 e 2 o elétron é excitado do orbital HOMO-3 e para os complexos 3-4.4 o elétron é excitado do orbital HOMO, exceto para o complexo 4.3 quando é excitado a partir do orbital HOMO-2. Notamos que para os complexos 3 e $\mathbf{4}$ o orbital HOMO apresenta contribuição do iodeto contudo essa contribuição é pequena como observado pela variação da densidade eletrônica entre o estado fundamental e excitado $\Delta \rho$ (ver Figura 33). O aumento da intensidade e a redução da energia de transição dos complexos $\mathbf{3}$ e $\mathbf{4}$ para os complexos $\mathbf{1}$ e $\mathbf{2}$ deve levar a um aumento de $\beta$. De fato, dentro da aproximação de dois níveis, as componentes de $\beta$ são diretamente proporcionais ao quadrado do momento de dipolo de transição e inversamente proporcional ao quadrado da energia de excitação. Embora os diferentes ânions não resultem em grandes diferenças no processo de A1F eles apresentam a tendência de aumentarem a energia de excitação com o tamanho do ânion. 


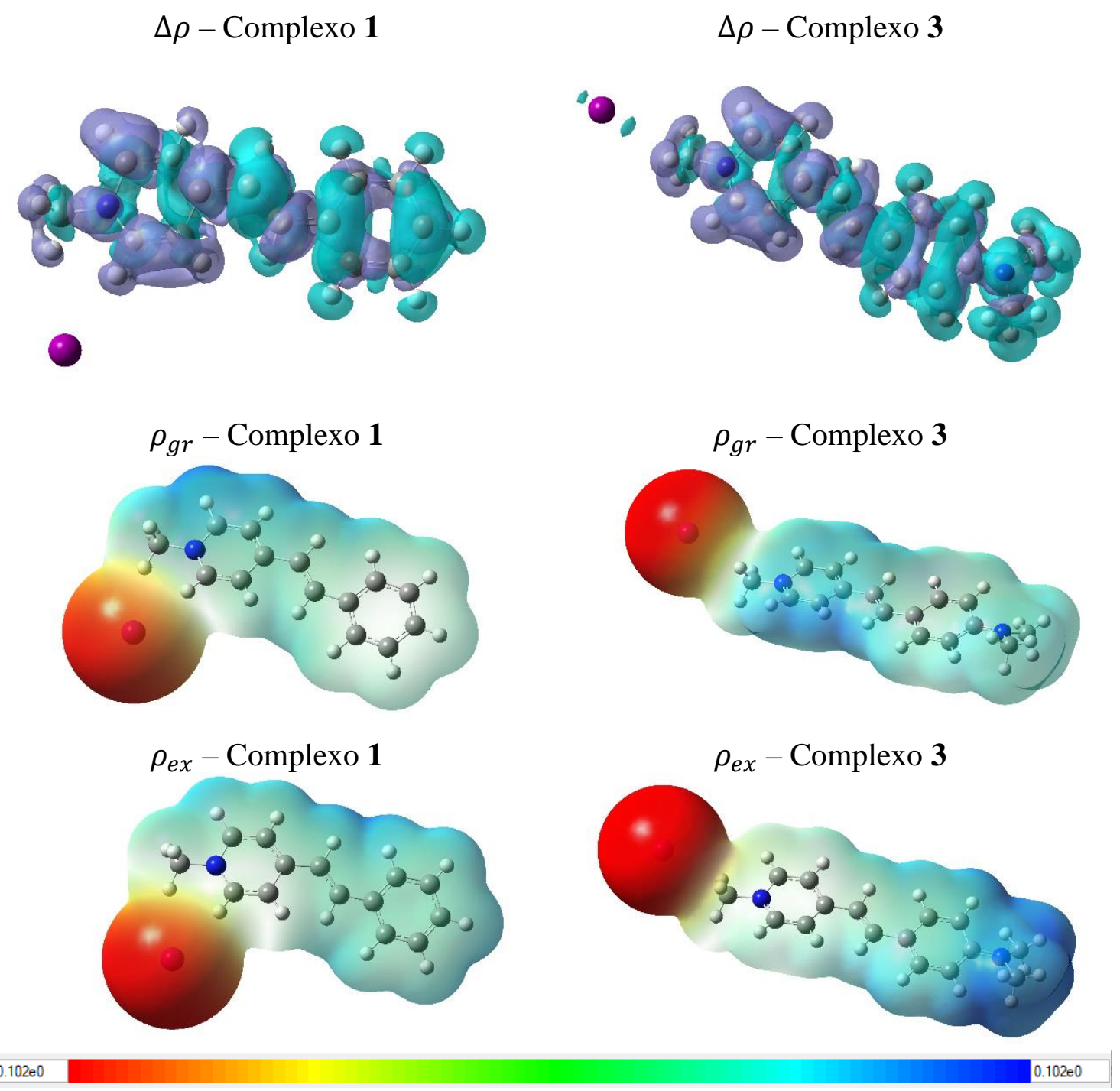

Figura 33. Diferença das densidades eletrônicas dos estados excitado e fundamental $\left(\Delta \rho=\rho_{\text {ex }}-\rho_{\text {gr }}\right)$ para os complexos 1 e 3 junto com as respectivas densidades $\rho_{\text {ex }}$ e $\rho_{\text {gr }}$. $\Delta \rho$ está representado por isosuperfícies de valor 0.0004 onde a cor azul corresponde a região de decréscimo da densidade eletrônica e a cor violeta representa a região de acréscimo da densidade eletrônica.

As energias de transição eletrônica reproduzem a mesma tendência experimental embora estejam deslocadas entre 0.1 a $0.4 \mathrm{eV}$ para maiores energias. Essas diferenças com os valores experimentais estão de acordo com análises de performance para o funcional M06-2X [172]. Em todos os casos, as energias de transição estão distantes das ressonâncias com o campo externo da radiação (1907 nm e 954 nm para o segundo harmônico) evitando efeitos indesejados nas respostas ONL.

Os valores calculados para as respostas EFISHG e HRS estão apresentados na Tabela 15 e na Tabela 16 onde as análises das diferentes contribuições de segunda e terceira ordem são apresentadas. De forma complementar, apresentamos na Figura 34 as distribuições dos valores de $\mu \beta_{/ /}$e da componente $\left[\mu \beta_{/ /}\right]_{x}$ obtidos para os diferentes complexos. Os valores médios de 
$\mu \beta_{/ /}$para os complexos $\mathbf{3}$ e $\mathbf{4}$ são uma ordem de magnitude maiores que os valores médios obtidos para os complexos 1 e $\mathbf{2}$. Este aumento é primeiramente atribuído à presença do grupo doador amino como terminal do anel, corroborando com o comportamento observado nos resultados de A1F. Além disso, essa tendência está de acordo com os dados experimentais [171]. De forma similar, o valor de $\beta_{/ /}$aumenta do complexo 1 para o complexo 4. Finalmente, pequenas diferenças foram observadas nos valores médios de $\mu$, a maior variação ( 20\%) é obtida entre os complexos 1 e 4 .

Os valores obtidos para o ângulo $\theta_{(\mu, \beta)}$ entre os vetores $\mu$ e $\beta$ são pequenos para os complexos com o grupo amino, levando a um aumento de $\beta_{/ /}$para os complexos $\mathbf{3}$ e $\mathbf{4}$ em comparação com os complexos 1 e 2. Considerando os complexos 4-4.4, os valores de $\beta_{/ /}$ aumentam seguindo a ordem $\mathrm{I}^{-}, \mathrm{ClO}_{4}^{-}, \mathrm{CF}_{3} \mathrm{SO}_{3}^{-}, \mathrm{SCN}^{-}$e pTS ${ }^{-}$, embora, considerando o desvio padrão da amostra todos os valores são estatisticamente equivalentes.

Tabela 15. Valores médios e desvios padrões para as respostas EFISHG e HRS obtidas em $1907 \mathrm{~nm}$.

\begin{tabular}{|ccccccc|}
\hline & $\begin{array}{c}\mu \beta_{/ /} \\
\left(10^{3} \text { u.a. }\right)\end{array}$ & $\begin{array}{c}\beta_{/ /} \\
(\text {u.a. })\end{array}$ & $\begin{array}{c}\mu \\
(\text { u.a. })\end{array}$ & $\begin{array}{c}\theta_{(\mu, \beta)} \\
\left({ }^{\circ}\right)\end{array}$ & $\begin{array}{c}\beta_{H R S} \\
(\text { u.a. })\end{array}$ & DR \\
\hline $\mathbf{1}^{*}$ & $12 \pm 17$ & $1377 \pm 1815$ & $8.50 \pm 0.89$ & $71.6 \pm 22.5$ & $3552 \pm 239$ & $3.97 \pm 0.41$ \\
$\mathbf{1}$ & $13 \pm 17$ & $1404 \pm 1895$ & $8.47 \pm 0.98$ & $73.5 \pm 21.3$ & $3908 \pm 616$ & $3.83 \pm 0.45$ \\
$\mathbf{2}$ & $28 \pm 25$ & $3087 \pm 2589$ & $8.64 \pm 0.92$ & $65.5 \pm 20.2$ & $5592 \pm 1024$ & $4.13 \pm 0.39$ \\
$\mathbf{3}$ & $164 \pm 72$ & $16574 \pm 6119$ & $9.64 \pm 1.01$ & $49.9 \pm 13.3$ & $18341 \pm 3699$ & $4.66 \pm 0.13$ \\
$\mathbf{4}$ & $187 \pm 109$ & $18210 \pm 9345$ & $9.81 \pm 1.20$ & $51.6 \pm 17.2$ & $20942 \pm 4856$ & $4.69 \pm 0.18$ \\
$\mathbf{4 . 1}$ & $198 \pm 81$ & $20209 \pm 6202$ & $9.53 \pm 1.35$ & $46.6 \pm 11.7$ & $20746 \pm 4091$ & $4.87 \pm 0.03$ \\
$\mathbf{4 . 2}$ & $186 \pm 96$ & $18563 \pm 7562$ & $9.68 \pm 1.41$ & $50.1 \pm 14.0$ & $20810 \pm 4173$ & $4.87 \pm 0.04$ \\
$\mathbf{4 . 3}$ & $215 \pm 93$ & $21203 \pm 6918$ & $9.78 \pm 1.51$ & $44.2 \pm 10.7$ & $20760 \pm 4691$ & $4.78 \pm 0.11$ \\
$\mathbf{4 . 4}$ & $180 \pm 75$ & $21721 \pm 6250$ & $8.05 \pm 1.48$ & $38.1 \pm 11.2$ & $19483 \pm 4188$ & $4.85 \pm 0.04$ \\
\hline
\end{tabular}

* Cálculos realizados para a simulação com geometria rígida.

Entre os complexos estudados, o 4.4 apresenta o maior valor de $\beta_{/ /}$e baixo valor de $\mu \beta_{/ /}$devido ao menor momento de dipolo observado para o complexo. Ainda, para os complexos 4 a 4.4, a diferença entre os valores médios de $\mu \beta / /$ são pequenas comparativamente com os respectivos desvios padrão. Os desvios padrão de $\mu \beta_{/ /}$representam respectivamente 131, 89 e $44 \%$ dos valores médios para os complexos 1-3 e 58, 41, 52, 43 e $42 \%$ para os complexos 4 a 4.4 .

Os valores de $\beta_{H R S}$ apresentam as mesmas tendências observadas para $\beta_{/ /}$e $\mu \beta_{/ /}$mas os desvios padrão são comparativamente menores (entre 16 e $23 \%$ ) que os valores médios. Note que para compostos com características ONL unidimensionais a relação $\beta_{/ /} \sim 1.45 \beta_{H R S}$ é 
Espectroscopia de Absorção de Dois Fótons em

Moléculas Orgânicas Incluindo Efeitos de Solvente

esperada. Este claramente não é o caso: os complexos $\mathbf{3}$ e $\mathbf{4}$ apresentam amplitudes similares; e os complexos 1 e 2 apresentam valores entre 0.35-0.55. A origem desses valores vem do fato dos vetores $\mu$ e $\beta$ não serem paralelos com grandes valores de $\theta_{(\mu, \beta)}$ e dos complexos não serem unidimensionais. Contudo, as maiores contribuições de $\mu \beta_{/ /}$são provenientes do produto $\mu_{x} \beta_{x}$. Além disso, notamos que os valores de DR são próximos de 5 para os complexos 3-4.4 corroborando com o caráter push-pull destes complexos.

Tabela 16. Valores médios e desvios padrões das respostas EFISHG obtidas em $1907 \mathrm{~nm} . R_{3 / 2}=\left(3 \mathrm{kT} \times \gamma_{/ /}\right) / \mu \beta_{/ /}$onde $T=298.15 \mathrm{~K}, 3 \mathrm{kT}=2.833 \times 10^{-3}$ u. $a$.

\begin{tabular}{|ccccccc|}
\hline & $\begin{array}{c}\mu / / / 3 k T \\
\left(10^{4} \text { u.a. }\right)\end{array}$ & $\begin{array}{c}\gamma_{/ /} \\
\left(10^{4} \text { u.a. }\right)\end{array}$ & $\begin{array}{c}\gamma_{E F I S H G} \\
\left(10^{4} \text { u.a. }\right)\end{array}$ & $R_{3 / 2}$ & $\begin{array}{c}{\left[\mu \beta_{/ /}\right]_{\text {eff }}} \\
\left(10^{-48} \text { esu }\right)\end{array}$ & $\begin{array}{c}{ }^{\mathrm{a}}\left[\mu \beta_{/ /}\right]_{\text {exp }} \\
\left(10^{-48} \mathrm{esu}\right)\end{array}$ \\
\hline $\mathbf{1}^{*}$ & $438 \pm 584$ & $60 \pm 8$ & $499 \pm 583$ & $0.064 \pm 0.767$ & $129 \pm 151$ & $800 ; 300 ; 170$ \\
$\mathbf{1}$ & $460 \pm 612$ & $73 \pm 15$ & $533 \pm 609$ & $-0.332 \pm 6.303^{\mathrm{b}}$ & $138 \pm 158$ & $800 ; 300 ; 170$ \\
$\mathbf{2}$ & $993 \pm 866$ & $97 \pm 16$ & $1091 \pm 868$ & $0.051 \pm 0.750$ & $283 \pm 225$ & $810 ; 600 ; 205$ \\
$\mathbf{3}$ & $5797 \pm 2526$ & $258 \pm 43$ & $6055 \pm 2550$ & $0.044 \pm 0.134$ & $1569 \pm 661$ & $1700 ; 1000 ; 1000$ \\
$\mathbf{4}$ & $6596 \pm 3831$ & $303 \pm 58$ & $6899 \pm 3863$ & $0.026 \pm 0.699$ & $1788 \pm 1001$ & $1900 ; 1400 ; 1090$ \\
$\mathbf{4 . 1}$ & $6973 \pm 2857$ & $272 \pm 48$ & $7245 \pm 2993$ & $0.045 \pm 0.034$ & $1878 \pm 747$ & $1150 ; 710 ; 225$ \\
$\mathbf{4 . 2}$ & $6567 \pm 3372$ & $273 \pm 48$ & $6840 \pm 3394$ & $0.047 \pm 0.046$ & $1773 \pm 880$ & $1950 ; 1200 ; 250$ \\
$\mathbf{4 . 3}$ & $7576 \pm 3267$ & $294 \pm 58$ & $7869 \pm 3303$ & $0.046 \pm 0.020$ & $2039 \pm 856$ & $1800 ; 1800 ; 1790$ \\
$\mathbf{4 . 4}$ & $6351 \pm 2658$ & $265 \pm 45$ & $6616 \pm 2685$ & $0.048 \pm 0.019$ & $1715 \pm 696$ & $1150 ; 780 ; 690$ \\
\hline
\end{tabular}

${ }^{a}$ Os resultados experimentais são dados para as respectivas concentrações: $1 \times 10^{-4} ; 5 \times 10^{-4}$; e $1 \times 10^{-3} \mathrm{M}$.

${ }^{b}$ Os valores de $R_{3 ; 2}$ obtidos considerando: $\operatorname{abs}\left(R_{3 / 2}\right)<5 \rightarrow R_{3 / 2}=0.012 \pm 0.725 ;$ abs $\left(R_{3 / 2}\right)<10 \rightarrow R_{3 / 2}=0.042 \pm$ $1.04 ;$ e $\operatorname{abs}\left(R_{3 / 2}\right) \neq 87.15 \rightarrow R_{3 / 2}=0.104 \pm 1.364$

* Cálculos realizados para a simulação com geometria rígida.

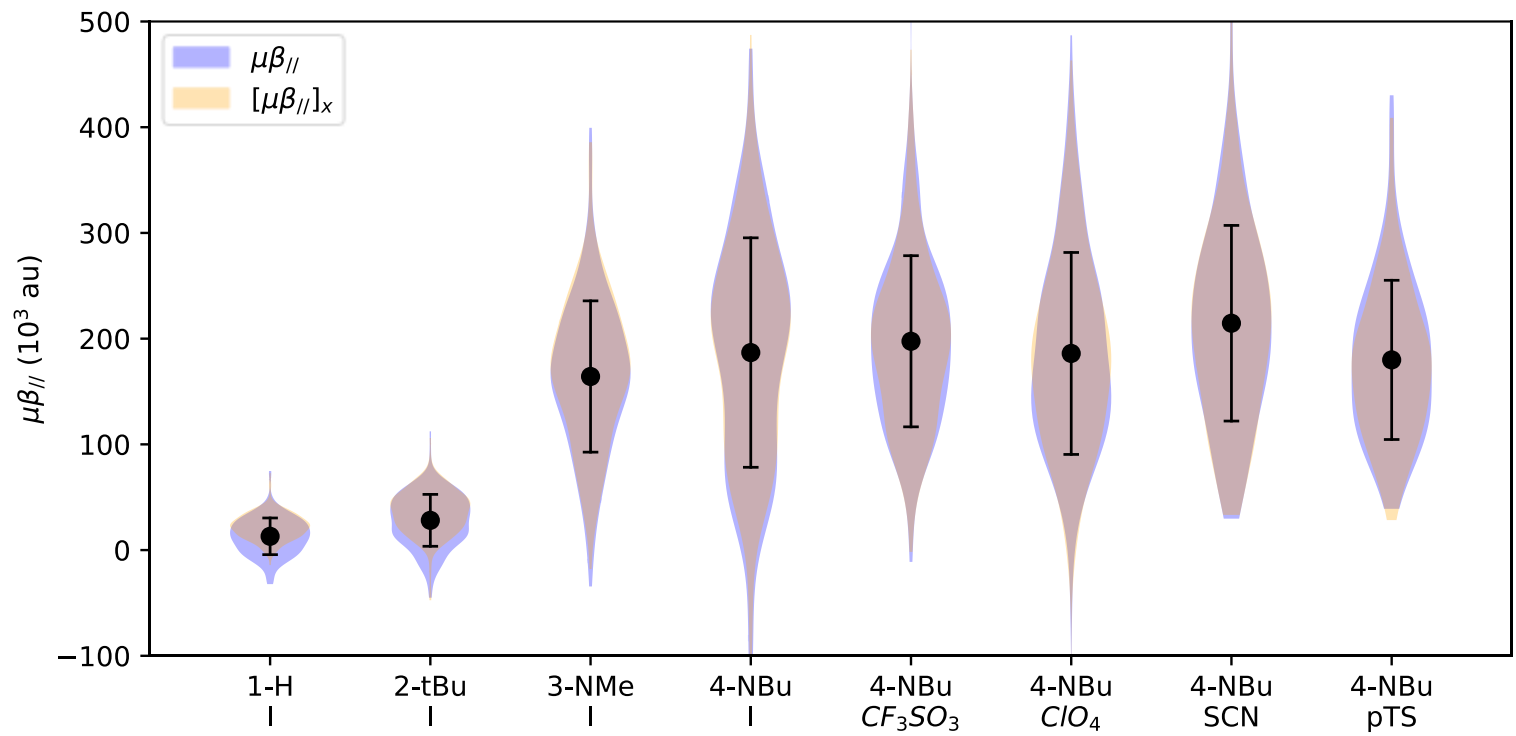

Figura 34 Distribuição violino dos resultados de $\mu \beta_{/ /}$e da componente $\left[\mu \beta_{/ /}\right]_{x}$ para os diferentes complexos, incluindo os valores médios (pontos pretos) e os desvios padrão (linhas pretas). 
Para separar os efeitos da flexibilidade interna dos cátions dos efeitos da mobilidade dos ânions sobre $\beta$ uma simulação adicional para o complexo 1 foi realizada considerando a geometria rígida, identificada com um asterisco $\mathbf{1}^{*}$. As distribuições violino ${ }^{3}$ evidenciam estes efeitos para $\mu \beta_{/ /}$e para $\beta_{H R S}$ e estão apresentadas na Figura 35. Distribuições similares foram obtidas para $\mu \beta_{/ /}$e para $\beta_{H R S}$ em ambas as simulações. Os valores médios de $\mu \beta_{/ /}$de $12 \times 10^{3} \mathrm{e}$ $13 \times 10^{3}$ u.a. foram obtidos respectivamente para as simulações $1^{*}$ e 1. Essa similaridade também foi observada para os valores de $\mu$ e de $\theta_{(\mu, \beta)}$. Também observamos poucas alterações nos valores de $\beta_{/ /}$e esperamos um comportamento similar para $\beta_{H R S}$. De fato, o valor médio de $\beta_{H R S}$ obtido para as geometrias rígidas são da ordem de $10 \%$ inferiores que o valor obtido para as geometrias flexíveis, porém o desvio padrão é duas vezes menor. Embora ambas as simulações não resultem em distribuições Gaussianas, os valores médio e mediana são praticamente equivalentes (ver Figura 35).
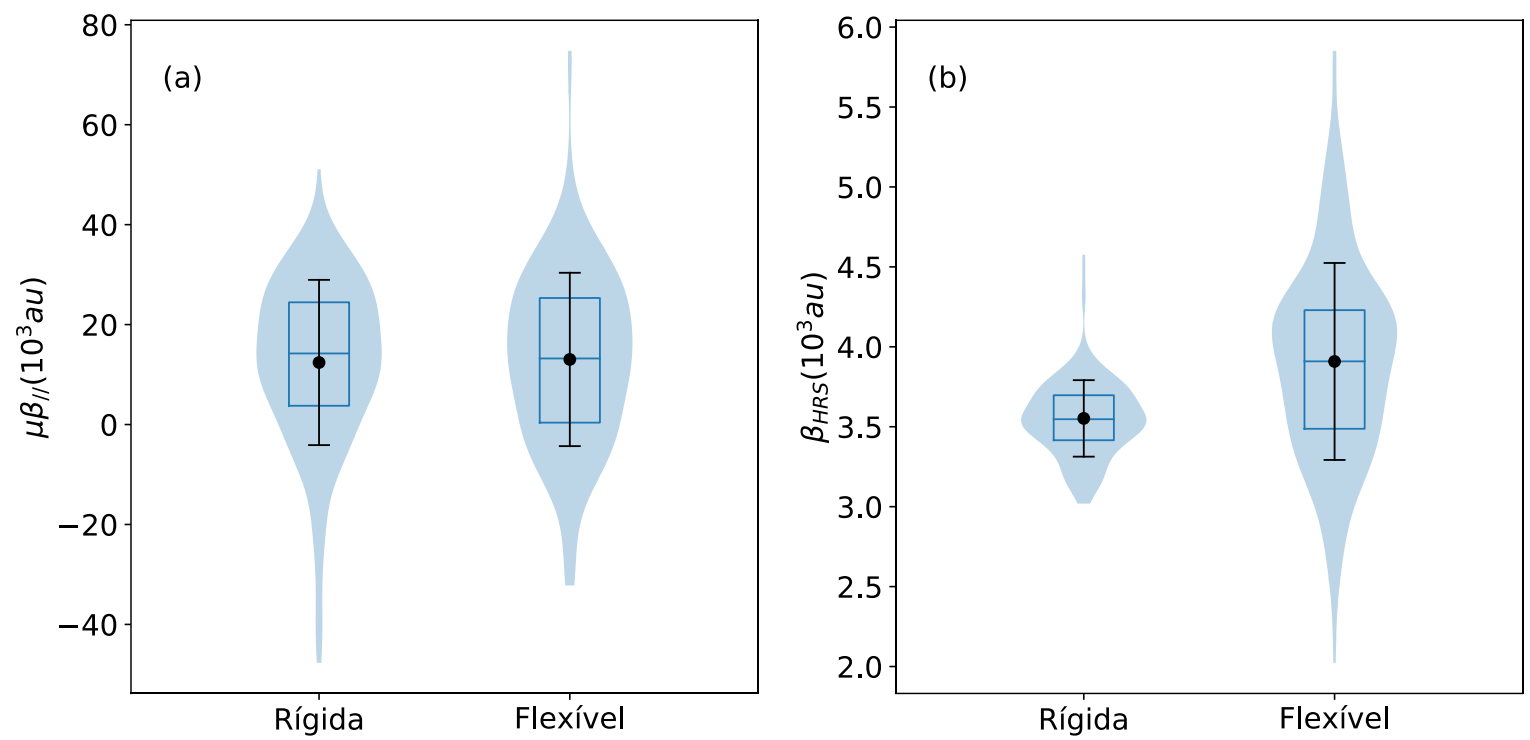

Figura 35. Distribuição violino dos resultados para $(a) \mu \beta_{/ /} e(b) \beta_{H R S}$, incluindo os valores médios (pontos pretos), desvio padrão (linhas pretas) e diagrama de caixa (linhas azuis indicando o primeiro quartil 25\%, a mediana e o terceiro quartil 75\%) para o complexo 1.

Uma vez que queremos compreender os efeitos dos ânions e da flexibilidade dos cátions é natural procurar por correlações entre as propriedades eletrônicas $\left(\beta_{x}, \beta_{H R S}\right.$, e $\left.\mu_{x}\right)$ e parâmetros geométricos (BLA e a projeção da coordenada $\mathrm{X}_{\mathrm{NA}}$ da distância $\mathrm{d}_{\mathrm{NA}}$ ). Análises da correlação linear de Pearson mostram fraca correlação entre $\beta_{x}$ versus BLA e $\beta_{H R S}$ versus BLA, embora $\beta_{H R S}$ apresente valores pouco maiores (ver Figura 36). O sinal negativo do coeficiente $\mathrm{R}$ indica um coeficiente linear negativo na regressão linear. Análises adicionais da correlação

\footnotetext{
${ }^{3}$ A distribuição violino representa a densidade de probabilidade espelhada e é uma forma mais clara para comparar a densidade de probabilidade com valores estatísticos da distribuição.
} 
de $\beta_{x}$ e $\beta_{H R S}$ versus as duas possibilidades de torções entre os dois anéis (rotações sobre as ligações $a$ e $c$ ) indicaram ausência de correlação, com parâmetros inferiores a 0.1. Essa fraca correlação e mesmo a ausência de correlação entre $\beta$ e parâmetros estruturais do cátion sugerem uma relação complexa que não pode ser descrita por análises lineares. Por outro lado, uma correlação quase perfeita foi observada entre $\mu_{x}$ e $\mathrm{X}_{\mathrm{NA}}$ para todos os complexos enquanto nenhuma correlação foi obtida entre $\beta_{x}$ e $X_{\mathrm{NA}}$, o que evidencia que a posição do ânion controla o momento de dipolo e dessa forma controla os valores de $\mu \beta_{/ /}$.
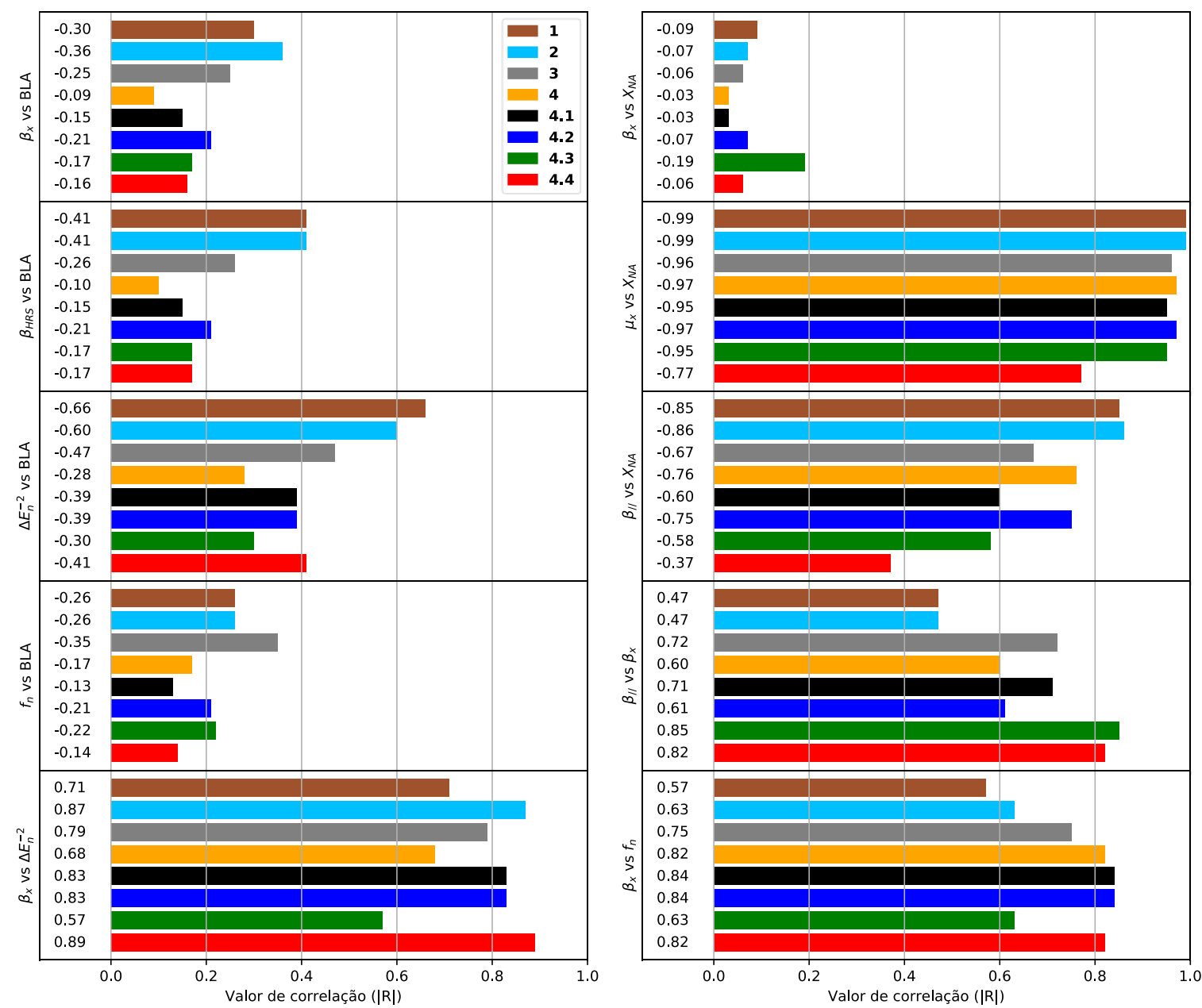

Figura 36. Correlação linear para propriedades moleculares $\left(\beta_{x}, \beta_{H R S}, \Delta E, f\right.$ e $\left.\mu_{x}\right)$ e os parâmetros geométricos (BLA e a projeção da coordenada $X_{N A}$ da distância $d_{N A}$ )

As correlações de BLA e de $\beta_{x}$ com os valores de $\Delta E$, mais especificamente $1 / \Delta E^{2}$, e de $f$, propriedades relacionadas com a excitação eletrônica intensa, também foram avaliadas. Estas comparações são úteis para estimar a contribuição da transição intensa utilizando a aproximação de dois estados, neste modelo simplificado podemos expressar $\beta_{x x x}=$ $\left(\mu_{e x}-\mu_{g r}\right)_{x} f_{x} / \Delta E^{2}$. Nós observamos forte correlação entre as propriedades ópticas lineares e 
não lineares indicando a predominância de dois estados na descrição de $\beta$. Além disso, as propriedades ópticas lineares são moderadamente correlacionadas com os valores de BLA.

No caso da resposta EFISHG, a amplitude global foi decomposta nas contribuições de segunda ordem $\left(\mu \beta_{/ /} / 3 k T\right)$ e de terceira ordem $\left(\gamma_{/ /}\right)$. Da mesma forma que observado para $\beta$ os valores de $\gamma_{/ /}$aumentam na presença do grupo doador amino. Os efeitos dos ânions sobre $\gamma_{/ /}$são pequenos. Ao incluir a contribuição de terceira ordem $\gamma_{/ /}$a resposta total EFISHG aumenta em $10 \%$ e $15 \%$ para os complexos 1 e 2 . Embora os valores de $\gamma_{/ /}$sejam maiores na presença do grupo amino, a resposta $\mu \beta_{/ /}$é ainda maior levando a razões $R_{3 / 2}$ no máximo de 5\%. Estes resultados estão apresentados na Tabela 16 e na Figura 37. Para o complexo 1, parte dos valores de $\theta_{(\mu, \beta)}$ são próximos de $90^{\circ}$ levando a menores contribuições de $\mu \beta_{/ /}$, em especial, quando $\theta_{(\mu, \beta)}=90.034^{\circ}$ o valor de $R_{3 / 2}$ é de -87.15 devido ao pequeno, mas negativo, valor de $\mu \beta_{/ /}$. Assim, este grande valor de $R_{3 / 2}$ pode levar a conclusões imprecisas e obtemos $R_{3 / 2}=0.104 \pm 1.364$ quando negligenciamos este caso.

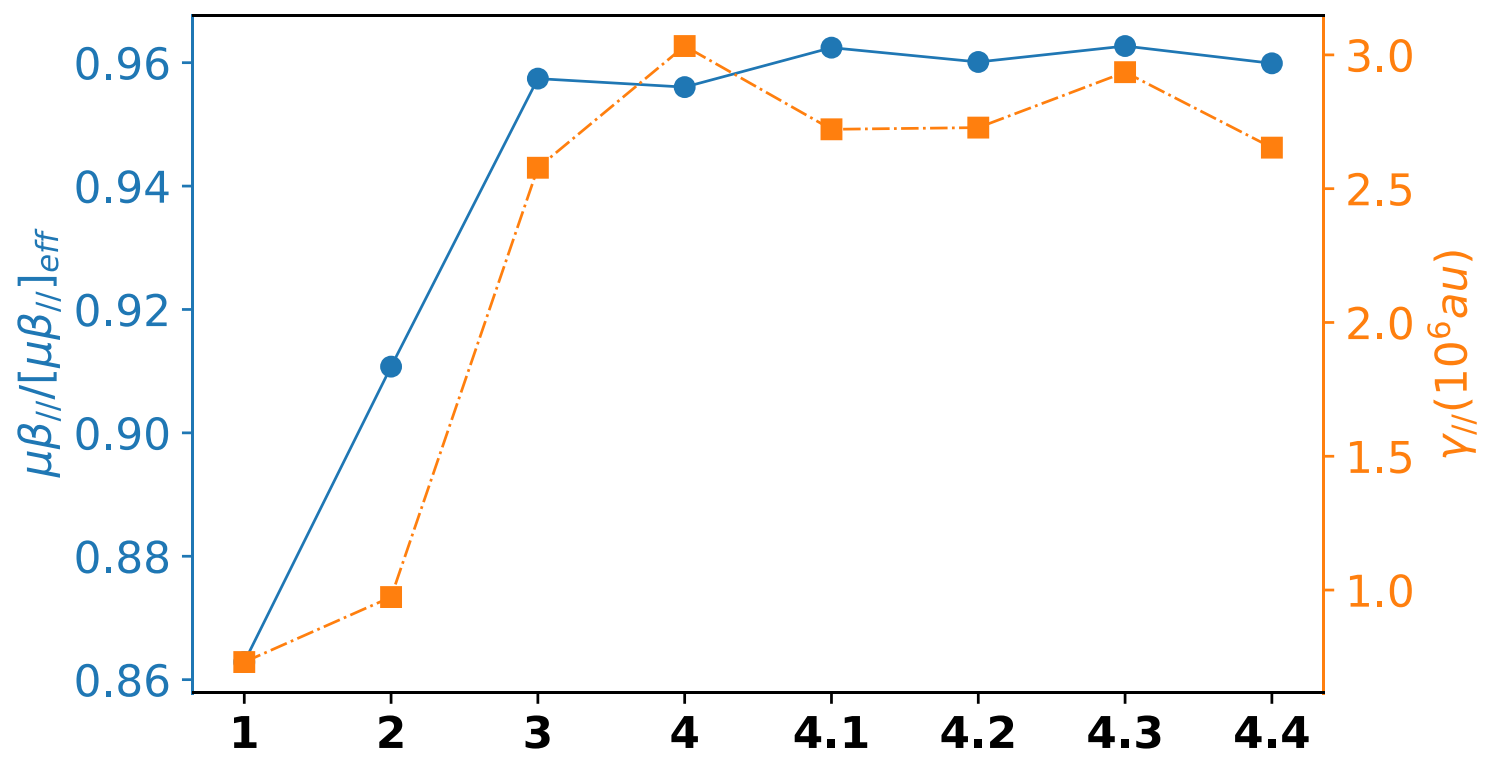

Figura 37. Razão entre as respostas EFISHG considerando ou não os efeitos de terceira ordem (em azul) e o termo de terceira ordem $\gamma_{/ /}$(em laranja).

Os valores EFISHG experimentais mostram sensibilidade devido a concentração dos complexos, dificultando comparações entre os resultados teóricos e experimentais. Essa variação dos resultados experimentais é tipicamente relacionada com a dissociação dos pares, que são dependentes das características dos ânions [171]. Dentro desta perspectiva, os resultados teóricos são contrastantes pois fornecem melhores concordâncias com os valores experimentais obtidos em altas concentrações $\left(1 \times 10^{-3} \mathrm{M}\right)$ para os complexos $\mathbf{1}$ e $\mathbf{2}$, enquanto as 
melhores concordâncias para os complexos $\mathbf{3}$ e $\mathbf{4}$ são observadas em baixas concentrações $\left(1 \times 10^{-3} \mathrm{M}\right)$.

\subsubsection{Conclusões}

Mostramos que nessa classe de complexos formados por pares iônicos a resposta EFISHG é efetivamente modulada pela distribuição espacial do ânion ao redor do cátion. Essa modulação ocorre devido às variações do momento de dipolo e não devido às variações das componentes de $\beta$. Esse comportamento foi demonstrado comparando os resultados obtidos das simulações moleculares com geometrias rígidas e flexíveis e de análises de correlação linear envolvendo as propriedades eletrônicas e os parâmetros geométricos dos complexos. Por ser independente do momento de dipolo a resposta HRS não foi modulada pela distribuição espacial do ânion e, consequentemente, apresenta maior sensibilidade às variações de BLA. A inclusão da contribuição de terceira ordem $\gamma_{/ /}$aumenta a resposta EFISHG em 15\% para os complexos 1 e 2 e em 5\% para os complexos 3-4.4 independentemente das características dos ânions.

Simulações moleculares multiescalas foram essenciais para incluir a distribuição do ânion ao redor do cátion e para incluir as variações da geometria provenientes de efeitos térmicos. Além disso, demonstramos a possiblidade de simular propriedades ONL para compostos formados por pares iônicos, embora considerar os efeitos de dissociações ainda seja desafiador. 


\section{Capítulo 4 Conclusões e Perspectivas}

Neste trabalho, investigamos diversos aspectos relacionados com o processo de A2F em moléculas orgânicas isoladas e em solução. Os efeitos de solventes foram incluídos utilizando modelos simples e não estruturados, como a aproximação PCM, até modelos com moléculas explícitas de solvente utilizando simulações moleculares clássicas e quânticas.

Diferentes formas de construir o espectro de A2F e obter o valor da seção de choque de A2F foram consideradas para cálculos multiescala S-QM/MM. Uma nova proposta de ajuste para a obtenção da largura total da banda de A2F, considerando a contribuição das configurações extraídas das simulações moleculares, resultou em melhores descrições do valor máximo da seção de choque de $\mathrm{A} 2 \mathrm{~F}$ e da intensidade de $\mathrm{A} 2 \mathrm{~F}$ relativa entre diferentes solventes. Essa proposta de atribuir o valor do alargamento espectral de forma não arbitrária será útil principalmente no desenvolvimento de novos compostos para aplicações baseadas no processo de A2F.

Mostramos que é possível utilizar campos elétricos externos para modular a intensidade da A2F. Mais do que isso, para um dos compostos considerados no estudo realizado observamos um aumento da intensidade da $\mathrm{A} 2 \mathrm{~F}$ ao mesmo tempo que uma redução da intensidade de $\mathrm{A} 1 \mathrm{~F}$ é induzida. Tal resultado é particularmente interessante porque ilustra a possibilidade de inibir ou reduzir o efeito indesejável do engrandecimento por ressonância devido a A1F em aplicações baseadas no processo de A2F. Também confirmamos, de forma semi quantitativa, que maiores quantidades de carga eletrônica transferida durante a excitação e deslocadas por maiores distâncias levam à maiores valores da seção de choque de A2F.

A obtenção teórica da seção de choque de A2F para sistemas moleculares grandes é um desafio computacional mesmo com a disponibilidade computacional atual. A utilização do método semi-empírico INDO/S, considerando excitações simples e duplas, descreveu com maior precisão as intensidades absolutas e relativas do espectro de A2F comparativamente com os resultados obtidos com o método de teoria resposta quadrática para TD-DFT. Assim, aliado ao excelente custo benefício, utilizar métodos semi-empíricos para a obtenção da seção de 
choque de A2F permite estudar moléculas inacessíveis a métodos mais rigorosos podendo, ainda, fornecer descrições mais realistas.

Além de todo o enfoque nas propriedades ópticas relacionadas com o processo de A2F também estudamos os efeitos não lineares longe de ressonâncias durante estágio na Universidade de Namur - Bélgica. Mostramos, com simulações moleculares de pares iônicos em meio líquido, que a resposta EFISHG é modulada pela posição relativa entre os íons. Esta modulação é controlada pela variação da direção do momento de dipolo causado pela distribuição dos sítios atômicos carregados.

Neste trabalho avaliamos diferentes metodologias para obter a seção de choque de A2F de moléculas orgânicas em solução. Avaliamos diferentes formas de obter o espectro de A2F utilizando simulações moleculares, computamos os efeitos devido às perturbações com campos eletrostáticos e exploramos o uso de métodos com baixo custo computacional para descrever as propriedades eletrônicas relacionadas com o processo de $\mathrm{A} 2 \mathrm{~F}$ de moléculas orgânicas grandes em solução. Uma vez que aplicações envolvendo biomoléculas ocorre geralmente em meios líquidos, e que tais sistemas apresentam elevada quantidade de átomos, é natural a perspectiva de combinar o conhecimento adquirido para simular a seção de choque de A2F nesse cenário de forma computacionalmente eficiente. 


\section{REFERÊNCIAS}

[1] Göppert-Mayer, M. Über Elementarakte Mit Zwei Quantensprüngen. Ann. der Phys. 1931, 401, 273.

[2] Göppert-Mayer, M. Elementary Processes with Two Quantum Transitions. Ann. der Phys. 2009, 18 (7-8), 466-479. https://doi.org/10.1002/andp.200910358.

[3] Kaiser, W.; Garrett, C. Two-Photon Excitation in $\mathrm{CaF}_{2}$ : Eu ${ }^{2+}$. Phys. Rev. Lett. 1961, 7 (6), 229-231. https://doi.org/10.1103/PhysRevLett.7.229.

[4] Parthenopoulos, D. A.; Rentzepis, P. M. Three-Dimensional Optical Storage Memory. Science 1989, 245 (4920), 843-845. https://doi.org/10.1126/science.245.4920.843.

[5] Li, L.; Wang, P.; Hu, Y.; Lin, G.; Wu, Y.; Huang, W.; Zhao, Q. Novel Carbazole Derivatives with Quinoline Ring: Synthesis, Electronic Transition, and Two-Photon Absorption Three-Dimensional Optical Data Storage. Spectrochim. Acta Part A Mol. Biomol. Spectrosc. 2015, 139, 243-252. https://doi.org/10.1016/j.saa.2014.10.122.

[6] Chen, Y.; Yang, X.; Gao, J. 3D Janus Plasmonic Helical Nanoapertures for PolarizationEncrypted Data Storage. Light Sci. Appl. 2019, 8 (1), 45. https://doi.org/10.1038/s41377019-0156-8.

[7] Denk, W.; Strickler, J. H.; Webb, W. W. Two-Photon Laser Scanning Fluorescence Microscopy. Science 1990, 248 (4951), 73-76. https://doi.org/10.1126/science.2321027.

[8] Zhang, H.; Wang, K.; Xuan, X.; Lv, Q.; Nie, Y.; Guo, H. Cancer Cell-Targeted TwoPhoton Fluorescence Probe for the Real-Time Ratiometric Imaging of DNA Damage. Chem. Commun. 2016, 52 (37), 6308-6311. https://doi.org/10.1039/C6CC02290A.

[9] Larson, D. R.; Zipfel, W. R.; Williams, R. M.; Clark, S. W.; Bruchez, M. P.; Wise, F. W.; Webb, W. W. Water-Soluble Quantum Dots for Multiphoton Fluorescence Imaging in Vivo. Science 2003, $300 \quad$ (5624), $1434-1436$. https://doi.org/10.1126/science.1083780.

[10] Kawata, S.; Sun, H. B.; Tanaka, T.; Takada, K. Finer Features for Functional Microdevices. Nature 2001, 412 (6848), 697-698. https://doi.org/10.1038/35089130.

[11] Bhawalkar, J. D.; He, G. S.; Prasad, P. N. Nonlinear Multiphoton Processes in Organic and Polymeric Materials. Reports Prog. Phys. 1996, 59, 1041-1070. https://doi.org/10.1088/0034-4885/59/9/001.

[12] Schmitt, J.; Heitz, V.; Sour, A.; Bolze, F.; Ftouni, H.; Nicoud, J.-F.; Flamigni, L.; Ventura, B. Diketopyrrolopyrrole-Porphyrin Conjugates with High Two-Photon Absorption and Singlet Oxygen Generation for Two-Photon Photodynamic Therapy. Angew. Chemie Int. Ed. 2015, 54 (1), 169-173. https://doi.org/10.1002/anie.201407537.

[13] Morel, Y.; Irimia, A.; Najechalski, P.; Kervella, Y.; Stephan, O.; Baldeck, P. L.; Andraud, C. Two-Photon Absorption and Optical Power Limiting of Bifluorene Molecule. J. Chem. Phys. 2001, 114 (12), 5391-5396. https://doi.org/10.1063/1.1351160.

[14] Abbotto, A.; Beverina, L.; Bradamante, S.; Facchetti, A.; Pagani, G. A.; Bozio, R.; Ferrante, C.; Pedron, D.; Signorini, R. Design and Synthesis of Heterocyclic MultiBranched Dyes for Two-Photon Absorption. Synth. Met. 2003, 139 (3), 795-797. https://doi.org/10.1016/S0379-6779(03)00281-9.

[15] Kanis, D. R.; Ratner, M. A.; Marks, T. J. Design and Construction of Molecular 
Assemblies with Large Second-Order Optical Nonlinearities. Quantum Chemical Aspects. Chem. Rev. 1994, 94 (1), 195-242. https://doi.org/10.1021/cr00025a007.

[16] Thanthiriwatte, K. S.; Nalin de Silva, K. M. Non-Linear Optical Properties of Novel Fluorenyl Derivatives - Ab Initio Quantum Chemical Calculations. J. Mol. Struct. 2002, 617 (1-3), 169-175. https://doi.org/10.1016/S0166-1280(02)00419-0.

[17] Silva, D. L.; Fonseca, R. D.; Vivas, M. G.; Ishow, E.; Canuto, S.; Mendonca, C. R.; De Boni, L. Experimental and Theoretical Investigation of the First-Order Hyperpolarizability of a Class of Triarylamine Derivatives. J. Chem. Phys. 2015, 142 (6), 064312. https://doi.org/10.1063/1.4906893.

[18] He, X.; Xu, B.; Liu, Y.; Yang, Y.; Tian, W. Effect of Intramolecular Charge Transfer on the Two-Photon Absorption Behavior of Multibranched Triphenylamine Derivations. $J$. Appl. Phys. 2012, 111 (5), 053516. https://doi.org/10.1063/1.3692076.

[19] Boyd, R. W. Nonlinear Optics, 3rd ed.; Academic Press: London, 2008.

[20] Kamada, K.; Ohta, K.; Iwase, Y.; Kondo, K. Two-Photon Absorption Properties of Symmetric Substituted Diacetylene: Drastic Enhancement of the Cross Section near the One-Photon Absorption Peak. Chem. Phys. Lett. 2003, 372 (3-4), 386-393. https://doi.org/10.1016/S0009-2614(03)00413-5.

[21] Campagnola, P. J.; Loew, L. M. Second-Harmonic Imaging Microscopy for Visualizing Biomolecular Arrays in Cells, Tissues and Organisms. Nat. Biotechnol. 2003, 21 (11), 1356-1360. https://doi.org/10.1038/nbt894.

[22] Andréasson, J.; Pischel, U. Molecules with a Sense of Logic: A Progress Report. Chem. Soc. Rev. 2015, 44 (5), 1053-1069. https://doi.org/10.1039/C4CS00342J.

[23] 3D optical data storage https://en.wikipedia.org/wiki/3D_optical_data_storage (accessed Sep 11, 2019).

[24] Tribuzi, V.; Otuka, A. 3D Laser microfabrication on polymeric materials http://www.fotonica.ifsc.usp.br/news/gazette.php?id=131009101010-US (accessed Sep 11, 2019).

[25] 2-photon imaging http://mcb.berkeley.edu/labs2/robey/content/2-photon-imaging (accessed Sep 11, 2019).

[26] Two-Photon Excitation (TPE) https://www.picoquant.com/applications/category/lifescience/two-photon-excitation (accessed Sep 11, 2019).

[27] Levine, B. F.; Bethea, C. G. Second and Third Order Hyperpolarizabilities of Organic Molecules. J. Chem. Phys. 1975, 63 (6), 2666-2682. https://doi.org/10.1063/1.431660.

[28] Alain, V.; Blanchard-Desce, M.; Ledoux-Rak, I.; Zyss, J. Amphiphilic Polyenic PushPull Chromophores for Nonlinear Optical Applications. Chem. Commun. 2000, 6 (5), 353-354. https://doi.org/10.1039/a908717f.

[29] Cariati, E.; Dragonetti, C.; Lucenti, E.; Nisic, F.; Righetto, S.; Roberto, D.; Tordin, E. An Acido-Triggered Reversible Luminescent and Nonlinear Optical Switch Based on a Substituted Styrylpyridine: EFISH Measurements as an Unusual Method to Reveal a Protonation-Deprotonation NLO Contrast. Chem. Commun. 2014, 50 (13), 1608-1610. https://doi.org/10.1039/c3cc48149b.

[30] Pielak, K.; Tonnelé, C.; Sanguinet, L.; Cariati, E.; Righetto, S.; Muccioli, L.; Castet, F.; Champagne, B. Dynamical Behavior and Second Harmonic Generation Responses in Acido-Triggered Molecular Switches. J. Phys. Chem. C 2018, 122, 26160-26168. https://doi.org/10.1021/acs.jpcc.8b08697.

[31] Woo, H. Y.; Hong, J. W.; Liu, B.; Mikhailovsky, A.; Korystov, D.; Bazan, G. C. WaterSoluble [2.2]Paracyclophane Chromophores with Large Two-Photon Action Cross Sections. J. Am. Chem. Soc. 2005, 127 (3), 820-821. https://doi.org/10.1021/ja0440811.

[32] Johnsen, M.; Ogilby, P. R. Effect of Solvent on Two-Photon Absorption by Vinyl Benzene Derivatives. J. Phys. Chem. A 2008, 112 (34), 7831-7839. 
https://doi.org/10.1021/jp8020326.

[33] Nag, A.; Goswami, D. Solvent Effect on Two-Photon Absorption and Fluorescence of Rhodamine Dyes. J. Photochem. Photobiol. A Chem. 2009, 206 (2-3), 188-197. https://doi.org/10.1016/j.jphotochem.2009.06.007.

[34] Frediani, L.; Rinkevicius, Z.; Ågren, H. Two-Photon Absorption in Solution by Means of Time-Dependent Density-Functional Theory and the Polarizable Continuum Model. J. Chem. Phys. 2005, 122 (24), 244104. https://doi.org/10.1063/1.1944727.

[35] Zhao, K.; Ferrighi, L.; Frediani, L.; Wang, C. K.; Luo, Y. Solvent Effects on Two-Photon Absorption of Dialkylamino Substituted Distyrylbenzene Chromophore. J. Chem. Phys. 2007, 126 (20). https://doi.org/10.1063/1.2740641.

[36] Chandra Jha, P.; Wang, Y.; Ågren, H. Two-Photon Absorption Cross-Sections of Reference Dyes: A Critical Examination. ChemPhysChem 2008, 9 (1), 111-116. https://doi.org/10.1002/cphc.200700397.

[37] Vivas, M. G.; Silva, D. L.; De Boni, L.; Bretonniere, Y.; Andraud, C.; Laibe-Darbour, F.; Mulatier, J. C.; Zaleśny, R.; Bartkowiak, W.; Canuto, S.; et al. Revealing the Electronic and Molecular Structure of Randomly Oriented Molecules by Polarized TwoPhoton Spectroscopy. J. Phys. Chem. Lett. 2013, 4 (10), 1753-1759. https://doi.org/10.1021/jz4007004.

[38] Vivas, M. G.; Silva, D. L.; Malinge, J.; Boujtita, M.; Zaleśny, R.; Bartkowiak, W.; Ågren, H.; Canuto, S.; De Boni, L.; Ishow, E.; et al. Molecular Structure - Optical Property Relationships for a Series of Non-Centrosymmetric Two-Photon Absorbing Push-Pull Triarylamine Molecules. Sci. Rep. 2015, 4 (1), 4447. https://doi.org/10.1038/srep04447.

[39] Solvation Effects on Molecules and Biomolecules: Computational Methods and Applications (Challenges and Advances in Computational Chemistry and Physics); Canuto, S., Ed.; Springer, 2008.

[40] Murugan, N. A.; Kongsted, J.; Rinkevicius, Z.; Aidas, K.; Mikkelsen, K. V; Ågren, H. Hybrid Density Functional Theory/Molecular Mechanics Calculations of Two-Photon Absorption of Dimethylamino Nitro Stilbene in Solution. Phys. Chem. Chem. Phys. 2011, 13 (27), 12506. https://doi.org/10.1039/c1cp20611g.

[41] Silva, D. L.; Murugan, N. A.; Kongsted, J.; Rinkevicius, Z.; Canuto, S.; Ågren, H. The Role of Molecular Conformation and Polarizable Embedding for One- and Two-Photon Absorption of Disperse Orange 3 in Solution. J. Phys. Chem. B 2012, 116 (28), 81698181. https://doi.org/10.1021/jp3032034.

[42] Silva, D. L.; Barreto, R. C.; Lacerda, E. G.; Coutinho, K.; Canuto, S. One- and TwoPhoton Absorption of Fluorescein Dianion in Water: A Study Using S-QM/MM Methodology and ZINDO Method. Spectrochim. Acta - Part A Mol. Biomol. Spectrosc. 2014, 119, 63-75. https://doi.org/10.1016/j.saa.2013.04.035.

[43] Paterson, M. J.; Kongsted, J.; Christiansen, O.; Mikkelsen, K. V.; Nielsen, C. B. TwoPhoton Absorption Cross Sections: An Investigation of Solvent Effects. Theoretical Studies on Formaldehyde and Water. J. Chem. Phys. 2006, 125 (18), 184501. https://doi.org/10.1063/1.2363997.

[44] Nielsen, C. B.; Christiansen, O.; Mikkelsen, K. V.; Kongsted, J. Density Functional SelfConsistent Quantum Mechanics/Molecular Mechanics Theory for Linear and Nonlinear Molecular Properties: Applications to Solvated Water and Formaldehyde. J. Chem. Phys. 2007, 126 (15), 154112. https://doi.org/10.1063/1.2711182.

[45] Cardenuto, M. H.; Champagne, B. QM/MM Investigation of the Concentration Effects on the Second-Order Nonlinear Optical Responses of Solutions. J. Chem. Phys. 2014, 141, 234104. https://doi.org/10.1063/1.4903545.

[46] Cardenuto, M. H.; Champagne, B. The First Hyperpolarizability of Nitrobenzene in 
Benzene Solutions: Investigation of the Effects of Electron Correlation within the Sequential QM/MM Approach. Phys. Chem. Chem. Phys. 2015, 17 (36), 23634-23642. https://doi.org/10.1039/C5CP03455H.

[47] Quertinmont, J.; Champagne, B.; Castet, F.; Cardenuto, M. H. Explicit versus Implicit Solvation Effects on the First Hyperpolarizability of an Organic Biphotochrome. J. Phys. Chem. A 2015, 119 (21), 5496-5503. https://doi.org/10.1021/acs.jpca.5b00631.

[48] Hayek, A.; Bolze, F.; Nicoud, J.-F.; Duperray, A.; Grichine, A.; Baldeck, P. L.; Vial, J.C. Two-Photon Water-Soluble Dyes and Their Amine-Reactive Derivatives for TwoPhoton Bio-Imaging Applications. Nonlinear Opt. Quantum Opt. 2006, 35, 155-164.

[49] Shen, Y. R. The Principles of Nonlinear Optics; Wiley-Interscience, 1984.

[50] Non-Linear Optical Properties of Matter; Papadopoulos, M. G., Sadlej, A. J., Leszczynski, J., Eds.; Springer Netherlands: Dordrecht, 2006. https://doi.org/10.1007/14020-4850-5.

[51] Chemla, D. S. Nonlinear Optical Properties of Organic Molecules and Crystals; Academic Press Inc, 1987.

[52] Prasad, P. N.; Williams, D. J. Introduction to Nonlinear Optical Effects in Molecules and Polymers; Wiley-Interscience, 1991.

[53] Cronstrand, P.; Luo, Y.; Ågren, H. Multi-Photon Absorption of Molecules. Adv. Quantum Chem. 2005, 50, 1-22. https://doi.org/10.1016/S0065-3276(05)50001-7.

[54] Orr, B. J.; Ward, J. F. Perturbation Theory of the Non-Linear Optical Polarization of an Isolated System. Mol. Phys. 1971, 20 (3), 513-526. https://doi.org/10.1080/00268977100100481.

[55] Monson, P. R.; McClain, W. M. Polarization Dependence of the Two-Photon Absorption of Tumbling Molecules with Application to Liquid 1-Chloronaphthalene and Benzene. J. Chem. Phys. 1970, 53 (1), 29-37. https://doi.org/10.1063/1.1673778.

[56] McClain, W. M. Excited State Symmetry Assignment Through Polarized Two-Photon Absorption Studies of Fluids. J. Chem. Phys. 1971, 55 (6), 2789-2796. https://doi.org/10.1063/1.1676494.

[57] Birge, R. R.; Bennett, J. A.; Pierce, B. M.; Thomas, T. M. Two-Photon Spectroscopy of the Visual Chromophores. Evidence for a Lowest Excited ${ }^{1} \mathrm{Ag}_{\mathrm{g}}^{-}$-like $\pi-\pi *$ State in AllTrans-Retinol (Vitamin A). J. Am. Chem. Soc. 1978, 100 (5), 1533-1539. https://doi.org/10.1021/ja00473a035.

[58] Birge, R. R.; Pierce, B. M. A Theoretical Analysis of the Two-Photon Properties of Linear Polyenes and the Visual Chromophores. J. Chem. Phys. 1979, 70 (1), 165. https://doi.org/10.1063/1.437217.

[59] Beerepoot, M. T. P.; Friese, D. H.; List, N. H.; Kongsted, J.; Ruud, K. Benchmarking Two-Photon Absorption Cross Sections: Performance of CC2 and CAM-B3LYP. Phys. Chem. Chem. Phys. 2015, 17, 19306-19314. https://doi.org/10.1039/C5CP03241E.

[60] Ledoux, I.; Zyss, J. Influence of the Molecular Environment in Solution Measurements of the Second-Order Optical Susceptibility for Urea and Derivatives. Chem. Phys. 1982, 73 (1-2), 203-213. https://doi.org/10.1016/0301-0104(82)85161-6.

[61] Beaujean, P.; Champagne, B. Coupled Cluster Evaluation of the Second and Third Harmonic Scattering Responses of Small Molecules. Theor. Chem. Acc. 2018, 137 (4), 50. https://doi.org/10.1007/s00214-018-2219-y.

[62] Métodos de Química Teórica e Modelagem Molecular; Morgon, N. H., Coutinho, K., Eds.; Livraria da Física, 2007.

[63] Vianna, J. D. M.; Fazzio, A.; Canuto, S. Teoria Quântica de Moléculas e Sólidos; Livraria da Física, 2004.

[64] Szabo, A.; Ostlund, N. S. Modern Quantum Chemistry: Introduction to Advanced Electronic Structure Theory; Dover, 1996. 
[65] Jensen, F. Introduction to Computational Chemistry; Wiley, 1999.

[66] Koch, W.; Holthausen, M. C. A Chemist's Guide to Density Functional Theory; Wiley, 2001.

[67] Barone, V. Computational Strategies for Spectroscopy: From Small Molecules to Nano Systems; John Wiley \& Sons, 2011. https://doi.org/10.1002/9781118008720.

[68] Born, M.; Oppenheimer, R. Zur Quantentheorie Der Molekeln. Ann. Phys. 1927, 389 (20), 457-484. https://doi.org/10.1002/andp.19273892002.

[69] Slater, J. The Theory of Complex Spectra. Phys. Rev. 1929, 34 (10), 1293-1322. https://doi.org/10.1103/PhysRev.34.1293.

[70] Roothaan, C. C. J. New Developments in Molecular Orbital Theory. Rev. Mod. Phys. 1951, 23 (2), 69-89. https://doi.org/10.1103/RevModPhys.23.69.

[71] Pariser, R.; Parr, R. G. A Semi-Empirical Theory of the Electronic Spectra and Electronic Structure of Complex Unsaturated Molecules. I. J. Chem. Phys. 1953, 21 (3), 466-471. https://doi.org/10.1063/1.1698929.

[72] Pople, J. A.; Santry, D. P.; Segal, G. A. Approximate Self-Consistent Molecular Orbital Theory. I. Invariant Procedures. J. Chem. Phys 1965, 43 (10), 129. https://doi.org/10.1063/1.1701475.

[73] Bene, J. Del; Jaffé, H. H. Use of the CNDO Method in Spectroscopy. I. Benzene, Pyridine, and the Diazines. J. Chem. Phys. 1968, 48 (4), 1807-1813. https://doi.org/10.1063/1.1668915.

[74] Hohenberg, P.; Kohn, W. Inhomogeneous Electron Gas. Phys. Rev. 1964, 136 (1962), 1912-1919. https://doi.org/10.1103/PhysRev.136.B864.

[75] Kohn, W.; Sham, L. J. Self-Consistent Equations Including Exchange and Correlation Effects. Phys. Rev. 1965, 140 (1951). https://doi.org/10.1103/PhysRev.140.A1133.

[76] Becke, A. D. Density-Functional Thermochemistry. III. The Role of Exact Exchange. J. Chem. Phys. 1993, 98 (7), 5648-5652. https://doi.org/10.1063/1.464913.

[77] Becke, A. D. Density-Functional Exchange-Energy Approximation with Correct Asymptotic Behavior. Phys. Rev. A 1988, 38 (6), 3098-3100. https://doi.org/10.1103/PhysRevA.38.3098.

[78] Lee, C.; Yang, W.; Parr, R. G. Development of the Colle-Salvetti Correlation-Energy Formula into a Functional of the Electron Density. Phys. Rev. B 1988, 37 (2), 785-789. https://doi.org/10.1103/PhysRevB.37.785.

[79] Grimme, S.; Antony, J.; Ehrlich, S.; Krieg, H. A Consistent and Accurate Ab Initio Parametrization of Density Functional Dispersion Correction (DFT-D) for the 94 Elements H-Pu. J. Chem. Phys. 2010, 132 (15), 154104. https://doi.org/10.1063/1.3382344.

[80] Yanai, T.; Tew, D. P.; Handy, N. C. A New Hybrid Exchange-Correlation Functional Using the Coulomb-Attenuating Method (CAM-B3LYP). Chem. Phys. Lett. 2004, 393 (2004), 51-57. https://doi.org/10.1016/j.cplett.2004.06.011.

[81] Frisch, M. J.; Trucks, G. W.; Schlegel, H. B.; Scuseria, G. E.; Robb, M. A.; Cheeseman, J. R.; Scalmani, G.; Barone, V.; Mennucci, B.; Petersson, G. A.; et al. Gaussian09. 2009.

[82] Chai, J. Da; Head-Gordon, M. Long-Range Corrected Hybrid Density Functionals with Damped Atom-Atom Dispersion Corrections. Phys. Chem. Chem. Phys. 2008, 10, 66156620. https://doi.org/10.1039/b810189b.

[83] Chai, J. Da; Head-Gordon, M. Systematic Optimization of Long-Range Corrected Hybrid Density Functionals. J. Chem. Phys. 2008, 128, 088106. https://doi.org/10.1063/1.2834918.

[84] Becke, A. D. Density-Functional Thermochemistry .V. Systematic Optimization of Exchange-Correlation Functionals. J. Chem. Phys. 1997, 107 (1997), 8554-8560. https://doi.org/10.1063/1.475007. 
[85] Runge, E.; Gross, E. K. U. Density-Functional Theory for Time-Dependent Systems. Phys. Rev. Lett. 1984, 52 (12), 997-1000. https://doi.org/10.1103/PhysRevLett.52.997.

[86] Warshel, A.; Levitt, M. Theoretical Studies of Enzymic Reactions: Dielectric, Electrostatic and Steric Stabilization of the Carbonium Ion in the Reaction of Lysozyme. J. Mol. Biol. 1976, 103 (2), 227-249. https://doi.org/10.1016/0022-2836(76)90311-9.

[87] Coutinho, K.; Canuto, S. Solvent Effects from a Sequential Monte Carlo - Quantum Mechanical Approach. Adv. Quantum Chem. 1997, 28, 89-105. https://doi.org/10.1016/S0065-3276(08)60209-9.

[88] Coutinho, K.; Canuto, S.; Zerner, M. C. Monte Carlo-Quantum Mechanics Study of the Solvatochromic Shifts of the Lowest Transition of Benzene. J. Chem. Phys. 2000, 112 (22), 9874-9880. https://doi.org/10.1063/1.481624.

[89] Kirkwood, J. G. Theory of Solutions of Molecules Containing Widely Separated Charges with Special Application to Zwitterions. J. Chem. Phys. 1934, 2 (7), 351. https://doi.org/10.1063/1.1749489.

[90] Onsager, L. Electric Moments of Molecules in Liquids. J. Am. Chem. Soc. 1936, 58 (8), 1486-1493. https://doi.org/10.1021/ja01299a050.

[91] Tapia, O.; Goscinski, O. Self-Consistent Reaction Field Theory of Solvent Effects. Mol. Phys. 1975, 29 (6), 1653-1661. https://doi.org/10.1080/00268977500101461.

[92] Rivail, J.-L.; Rinaldi, D. A Quantum Chemical Approach to Dielectric Solvent Effects in Molecular Liquids. Chem. Phys. 1976, 18 (1-2), 233-242. https://doi.org/10.1016/0301-0104(76)87050-4.

[93] Miertuš, S.; Scrocco, E.; Tomasi, J. Electrostatic Interaction of a Solute with a Continuum. A Direct Utilizaion of Ab Initio Molecular Potentials for the Prevision of Solvent Effects. Chem. Phys. 1981, 55 (1), 117-129. https://doi.org/10.1016/03010104(81)85090-2.

[94] Tomasi, J.; Mennucci, B.; Cammi, R. Quantum Mechanical Continuum Solvation Models. Chem. Rev. 2005, 105 (8), 2999-3094. https://doi.org/10.1021/cr9904009.

[95] Metropolis, N.; Rosenbluth, A. W.; Rosenbluth, M. N.; Teller, A. H.; Teller, E. Equation of State Calculations by Fast Computing Machines. J. Chem. Phys. 1953, 21 (6), 10871092. https://doi.org/10.1063/1.1699114.

[96] Coutinho, K.; Canuto, S. DICE(v2.9) A Monte Carlo Program for Molecular Liquid Simulation, University of Sao Paulo, Brazil (2013).

[97] Cezar, H. M.; Canuto, S.; Coutinho, K. DICE(v3.0beta) A Monte Carlo Program for Molecular Liquid Simulation, University of Sao Paulo, Brazil (2018).

[98] Pranata, J.; Wierschke, S. G.; Jorgensen, W. L. OPLS Potential Functions for Nucleotide Bases. Relative Association Constants of Hydrogen-Bonded Base Pairs in Chloroform. J. Am. Chem. Soc. 1991, 113 (8), 2810-2819. https://doi.org/10.1021/ja00008a002.

[99] Jorgensen, W. L.; Maxwell, D. S.; Tirado-Rives, J. Development and Testing of the OPLS All-Atom Force Field on Conformational Energetics and Properties of Organic Liquids. J. Am. Chem. Soc. 1996, 118 (45), 11225-11236. https://doi.org/10.1021/ja9621760.

[100] Coutinho, K.; Guedes, R. C.; Cabral, B. J. C.; Canuto, S. Electronic Polarization of Liquid Water: Converged Monte Carlo-Quantum Mechanics Results for the Multipole Moments. Chem. Phys. Lett. 2003, 369 (3-4), 345-353. https://doi.org/10.1016/S00092614(02)02026-2.

[101] Ludwig, V.; Coutinho, K.; Canuto, S. A Monte Carlo-Quantum Mechanics Study of the Lowest $n-\pi *$ and $\pi-\pi *$ States of Uracil in Water. Phys. Chem. Chem. Phys. 2007, 9 (35), 4907-4912. https://doi.org/10.1039/b704335j.

[102] Cardenuto, M. H.; Coutinho, K.; Cabral, B. J. C.; Canuto, S. Electronic Properties in Supercritical Fluids. Adv. Quantum Chem. 2015, 71, 323-339. 
https://doi.org/10.1016/bs.aiq.2015.03.006.

[103] Silva, D. L.; Murugan, N. A.; Kongsted, J.; Ågren, H.; Canuto, S. Self-Aggregation and Optical Absorption of Stilbazolium Merocyanine in Chloroform. J. Phys. Chem. B 2014, 118 (7), 1715-1725. https://doi.org/10.1021/jp411178h.

[104] Verlet, L. Computer "Experiments" on Classical Fluids. I. Thermodynamical Properties of Lennard-Jones Molecules. Phys. Rev. 1967, 159 (1), 98-103. https://doi.org/10.1103/PhysRev.159.98.

[105] Hockney, R. W. Potential Calculation and Some Applications. Methods Comput. Phys. 1970, 9, 135-211.

[106] Swope, W. C.; Andersen, H. C.; Berens, P. H.; Wilson, K. R. A Computer Simulation Method for the Calculation of Equilibrium Constants for the Formation of Physical Clusters of Molecules: Application to Small Water Clusters. J. Chem. Phys. 1982, 76 (1), 637-649. https://doi.org/10.1063/1.442716.

[107] Coutinho, K.; Georg, H. C.; Fonseca, T. L.; Ludwig, V.; Canuto, S. An Efficient Statistically Converged Average Configuration for Solvent Effects. Chem. Phys. Lett. 2007, 437 (1-3), 148-152. https://doi.org/10.1016/j.cplett.2007.02.012.

[108] Le Bahers, T.; Adamo, C.; Ciofini, I. A Qualitative Index of Spatial Extent in ChargeTransfer Excitations. J. Chem. Theory Comput. 2011, 7 (8), 2498-2506. https://doi.org/10.1021/ct200308m.

[109] Jacquemin, D.; Le Bahers, T.; Adamo, C.; Ciofini, I. What Is the "Best" Atomic Charge Model to Describe through-Space Charge-Transfer Excitations? Phys. Chem. Chem. Phys. 2012, 14 (16), 5383-5388. https://doi.org/10.1039/c2cp40261k.

[110] Jacquemin, D. http://www.sciences.univ-nantes.fr/CEISAM/erc/marches/?p=973.(52) http://www.sciences.univ-nantes.fr/CEISAM/erc/marches/?p=973.(52).

[111] Bednarska, J.; Zaleśny, R.; Tian, G.; Murugan, N. A.; Ågren, H.; Bartkowiak, W. Nonempirical Simulations of Inhomogeneous Broadening of Electronic Transitions in Solution: Predicting Band Shapes in One- and Two-Photon Absorption Spectra of Chalcones. Molecules 2017, 22 (10), 1643. https://doi.org/10.3390/molecules22101643.

[112] Millefiori, S.; Favini, G.; Millefiori, A.; Grasso, D. Electronic Spectra and Structure of Nitroanilines. Spectrochim. Acta Part A Mol. Spectrosc. 1977, 33 (1), 21-27. https://doi.org/10.1016/0584-8539(77)80143-8.

[113] Kovalenko, S. A.; Schanz, R.; Farztdinov, V. M.; Hennig, H.; Ernsting, N. P. Femtosecond Relaxation of Photoexcited Para-Nitroaniline: Solvation, Charge Transfer, Internal Conversion and Cooling. Chem. Phys. Lett. 2000, 323 (3-4), 312-322. https://doi.org/10.1016/S0009-2614(00)00432-2.

[114] Yasukawa, T.; Kimura, T.; Uda, M. An AM1 Study of the Effects of Intermolecular Interactions on Hyperpolarizability of P-Nitroanilines. Chem. Phys. Lett. 1990, 169 (3), 259-262. https://doi.org/10.1016/0009-2614(90)85197-K.

[115] Luo, Y.; Ågren, H.; Vahtras, O.; Jørgensen, P. The Hyperpolarizability Dispersion of Para-Nitroaniline. Chem. Phys. Lett. 1993, 207 (2-3), 190-194. https://doi.org/10.1016/0009-2614(93)87013-S.

[116] Bartkowiak, W.; Lipiński, J. Solvent Effect on the Nonlinear Optical Properties of ParaNitroaniline Studied by Langevin Dipoles-Monte Carlo (LD/MC) Approach. Comput. Chem. 1998, 22 (1), 31-37. https://doi.org/10.1016/S0097-8485(97)00030-2.

[117] Jensen, L.; van Duijnen, P. T. The First Hyperpolarizability of P-Nitroaniline in 1,4Dioxane: A Quantum Mechanical/Molecular Mechanics Study. J. Chem. Phys. 2005, 123 (7), 074307. https://doi.org/10.1063/1.1999633.

[118] Shoute, L. C. T.; Helburn, R.; Kelley, A. M. Solvent Effects on the Resonance Raman and Hyper-Raman Spectra and First Hyperpolarizability of N,N-Dipropyl-pNitroaniline. J. Phys. Chem. A 2007, 111 (7), 1251-1258. 
https://doi.org/10.1021/jp066599s.

[119] Eriksen, J. J.; Sauer, S. P. A.; Mikkelsen, K. V.; Christiansen, O.; Jensen, H. J. A.; Kongsted, J. Failures of TDDFT in Describing the Lowest Intramolecular ChargeTransfer Excitation in Para-Nitroaniline. Mol. Phys. 2013, 111 (9-11), 1235-1248. https://doi.org/10.1080/00268976.2013.793841.

[120] Friese, D. H.; Hättig, C.; Ruud, K. Calculation of Two-Photon Absorption Strengths with the Approximate Coupled Cluster Singles and Doubles Model CC2 Using the Resolution-of-Identity Approximation. Phys. Chem. Chem. Phys. 2012, 14 (3), 11751184. https://doi.org/10.1039/c1cp23045j.

[121] Cabral, B. J. C.; Coutinho, K.; Canuto, S. A First-Principles Approach to the Dynamics and Electronic Properties of p -Nitroaniline in Water. J. Phys. Chem. A 2016, 120 (22), 3878-3887. https://doi.org/10.1021/acs.jpca.6b01797.

[122] Reis, H.; Grzybowski, A.; Papadopoulos, M. G. Computer Simulation of the Linear and Nonlinear Optical Susceptibilities of $\mathrm{p}$-Nitroaniline in Cyclohexane, 1,4-Dioxane, and Tetrahydrofuran in Quadrupolar Approximation. I. Molecular Polarizabilities and Hyperpolarizabilities. J. Phys. Chem. A 2005, 109 (44), 10106-10120. https://doi.org/10.1021/jp052875b.

[123] Beljonne, D.; Wenseleers, W.; Zojer, E.; Shuai, Z.; Vogel, H.; Pond, S. J. K.; Perry, J. W.; Marder, S. R.; Brédas, J. L. Role of Dimensionality on the Two-Photon Absorption Response of Conjugated Molecules: The Case of Octupolar Compounds. Adv. Funct. Mater. 2002, $12 \quad$ (9), 631-641. https://doi.org/10.1002/16163028(20020916)12:9<631::AID-ADFM631>3.0.CO;2-W.

[124] Rudberg, E.; Sałek, P.; Helgaker, T.; Ågren, H. Calculations of Two-Photon ChargeTransfer Excitations Using Coulomb-Attenuated Density-Functional Theory. J. Chem. Phys. 2005, 123 (18), 184108. https://doi.org/10.1063/1.2104367.

[125] Zaleśny, R.; Tian, G.; Hättig, C.; Bartkowiak, W.; Ågren, H. Toward Assessment of Density Functionals for Vibronic Coupling in Two-Photon Absorption: A Case Study of 4-Nitroaniline. J. Comput. Chem. 2015, 36 (15), 1124-1131. https://doi.org/10.1002/jcc.23903.

[126] Nanda, K. D.; Krylov, A. I. The Effect of Polarizable Environment on Two-Photon Absorption Cross Sections Characterized by the Equation-of-Motion Coupled-Cluster Singles and Doubles Method Combined with the Effective Fragment Potential Approach. J. Chem. Phys. 2018, 149 (16), 164109. https://doi.org/10.1063/1.5048627.

[127] Wielgus, M.; Michalska, J.; Samoc, M.; Bartkowiak, W. Two-Photon Solvatochromism III: Experimental Study of the Solvent Effects on Two-Photon Absorption Spectrum of p-Nitroaniline. Dye. Pigment. 2015, 113, 426-434. https://doi.org/10.1016/j.dyepig.2014.09.009.

[128] Rivelino, R.; Cabral, B. J. C.; Coutinho, K.; Canuto, S. Electronic Polarization in Liquid Acetonitrile: A Sequential Monte Carlo/Quantum Mechanics Investigation. Chem. Phys. Lett. 2005, 407 (1-3), 13-17. https://doi.org/10.1016/j.cplett.2005.03.049.

[129] Orozco-Gonzalez, Y.; Manathunga, M.; Marín, M. del C.; Agathangelou, D.; Jung, K.H.; Melaccio, F.; Ferré, N.; Haacke, S.; Coutinho, K.; Canuto, S.; et al. An Average Solvent Electrostatic Configuration Protocol for QM/MM Free Energy Optimization: Implementation and Application to Rhodopsin Systems. J. Chem. Theory Comput. 2017, 13 (12), 6391-6404. https://doi.org/10.1021/acs.jctc.7b00860.

[130] Luhmer, M.; Reisse, J. Molecular Dynamics Simulation Study of the NMR Relaxation of Xenon-131 Dissolved in 1,3-Dioxane and 1,4-Dioxane. J. Magn. Reson. Ser. A 1995, 115 (2), 197-205. https://doi.org/10.1006/jmra.1995.1167.

[131] Zheng, Y.; Ornstein, R. L. A Molecular Dynamics and Quantum Mechanics Analysis of the Effect of DMSO on Enzyme Structure and Dynamics: Subtilisin. J. Am. Chem. Soc. 
1996, 118 (17), 4175-4180. https://doi.org/10.1021/ja9539195.

[132] Vishnyakov, A.; Lyubartsev, A. P.; Laaksonen, A. Molecular Dynamics Simulations of Dimethyl Sulfoxide and Dimethyl Sulfoxide-Water Mixture. J. Phys. Chem. A 2001, 105 (10), 1702-1710. https://doi.org/10.1021/jp0007336.

[133] Berendsen, H. J. C.; Grigera, J. R.; Straatsma, T. P. The Missing Term in Effective Pair Potentials. J. Phys. Chem. 1987, 91 (24), 6269-6271. https://doi.org/10.1021/j100308a038.

[134] Canuto, S.; Coutinho, K. From Hydrogen Bond to Bulk: Solvation Analysis of the n-П* Transition of Formaldehyde in Water. Int. J. Quantum Chem. 2000, 77 (1), 192-198. https://doi.org/10.1002/(SICI)1097-461X(2000)77:1<192::AID-QUA18>3.0.CO;2-2.

[135] Coutinho, K.; Cabral, B. J. C.; Canuto, S. Can Larger Dipoles Solvate Less? SoluteSolvent Hydrogen Bond and the Differential Solvation of Phenol and Phenoxy. Chem. Phys. Lett. 2004, 399 (4-6), 534-538. https://doi.org/10.1016/j.cplett.2004.10.063.

[136] Martyna, G. J.; Tuckerman, M. E. A Reciprocal Space Based Method for Treating Long Range Interactions in Ab Initio and Force-Field-Based Calculations in Clusters. J. Chem. Phys. 1999, 110 (6), 2810-2821. https://doi.org/10.1063/1.477923.

[137] VandeVondele, J.; Hutter, J. Gaussian Basis Sets for Accurate Calculations on Molecular Systems in Gas and Condensed Phases. J. Chem. Phys. 2007, 127 (11), 114105. https://doi.org/10.1063/1.2770708.

[138] VandeVondele, J.; Krack, M.; Mohamed, F.; Parrinello, M.; Chassaing, T.; Hutter, J. Quickstep: Fast and Accurate Density Functional Calculations Using a Mixed Gaussian and Plane Waves Approach. Comput. Phys. Commun. 2005, 167 (2), 103-128. https://doi.org/10.1016/j.cpc.2004.12.014.

[139] Dalton, a molecular electronic structure program, Release Dalton2016.2 (2016), see http://daltonprogram.org http://daltonprogram.org.

[140] Aidas, K.; Angeli, C.; Bak, K. L.; Bakken, V.; Bast, R.; Boman, L.; Christiansen, O.; Cimiraglia, R.; Coriani, S.; Dahle, P.; et al. The Dalton Quantum Chemistry Program System. Wiley Interdisciplinary Reviews: Computational Molecular Science. May 2014, pp 269-284. https://doi.org/10.1002/wcms.1172.

[141] Sinha, H. K.; Thomson, P. C. P.; Yates, K. Excited State Dipole Moments and Polarizabilities of Some Aromatic Alkenes and Alkynes. Can. J. Chem. 1990, 68 (9), 1507-1513. https://doi.org/10.1139/v90-232.

[142] Sharma, D.; Sahoo, S.; Mishra, B. K. Molecular Modeling in Dioxane Methanol Interaction. J. Mol. Model. 2014, 20 (9), 2408. https://doi.org/10.1007/s00894-0142408-0.

[143] Albert, I. D. L.; Marks, T. J.; Ratner, M. A. Rational Design of Molecules with Large Hyperpolarizabilities. Electric Field, Solvent Polarity, and Bond Length Alternation Effects on Merocyanine Dye Linear and Nonlinear Optical Properties. J. Phys. Chem. 1996, 100 (23), 9714-9725. https://doi.org/10.1021/jp960860v.

[144] Manzoni, V.; Coutinho, K.; Canuto, S. An Insightful Approach for Understanding Solvatochromic Reversal. Chem. Phys. Lett. 2016, 655-656, 30-34. https://doi.org/10.1016/j.cplett.2016.05.028.

[145] Kogej, T.; Beljonne, D.; Meyers, F.; Perry, J. W.; Marder, S. R.; Brédas, J. L. Mechanisms for Enhancement of Two-Photon Absorption in Donor-Acceptor Conjugated Chromophores. Chem. Phys. Lett. 1998, 298 (1-3), 1-6. https://doi.org/10.1016/S0009-2614(98)01196-8.

[146] Meyers, F.; Marder, S. R.; Pierce, B. M.; Bredas, J. L. Electric Field Modulated Nonlinear Optical Properties of Donor-Acceptor Polyenes: Sum-Over-States Investigation of the Relationship between Molecular Polarizabilities $(\alpha, \beta, \gamma)$ and Bond Length Alternation. J. Am. Chem. Soc. 1994, 116 (23), 10703-10714. 
https://doi.org/10.1021/ja00102a040.

[147] Garcia, H.; Kalyanaraman, R. Phonon-Assisted Two-Photon Absorption in the Presence of a Dc-Field: The Nonlinear Franz-Keldysh Effect in Indirect Gap Semiconductors. $J$. Phys. B At. Mol. Opt. Phys. 2006, 39 (12), 2737-2746. https://doi.org/10.1088/09534075/39/12/009.

[148] Cui, H. Y.; Li, Z. F.; Liu, Z. L.; Wang, C.; Chen, X. S.; Hu, X. N.; Ye, Z. H.; Lu, W. Modulation of the Two-Photon Absorption by Electric Fields in HgCdTe Photodiode. Appl. Phys. Lett. 2008, 92 (2), 021128. https://doi.org/10.1063/1.2835049.

[149] Cui, H.; Yang, J.; Zeng, J.; Tang, Z. Optical Limiting Using the Two-Photon Absorption Electrical Modulation Effect in HgCdTe Photodiode. Sci. World J. 2013, 2013 (1), 4-7. https://doi.org/10.1155/2013/245310.

[150] Abe, J.; Nemoto, N.; Nagase, Y.; Shirai, Y. Ab Initio MO Investigations of Molecular Structures in the Ground and First Excited States of Heterocyclic Pyridinium Betaine. Chem. Phys. Lett. 1996, 261 (1-2), 18-22. https://doi.org/10.1016/0009-2614(96)00958$\mathrm{X}$.

[151] Paley, M. S.; Harris, J. M. Synthesis and Characterization of Some Pyridinium NPhenoxide Betaine Dyes for Second-Harmonic Generation. J. Org. Chem. 1991, 56 (2), 568-574. https://doi.org/10.1021/jo00002a017.

[152] Ishida, T.; Rossky, P. J. Solvent Effects on Solute Electronic Structure and Properties: Theoretical Study of a Betaine Dye Molecule in Polar Solvents. J. Phys. Chem. A 2001, 105 (3), 558-565. https://doi.org/10.1021/jp0041104.

[153] Hernandes, M. Z.; Longo, R.; Coutinho, K.; Canuto, S. Solute Relaxation on the Solvatochromism of Ortho-Betaine Dyes. A Sequential Monte Carlo/Quantum Mechanics Study. Phys. Chem. Chem. Phys. 2004, 6 (9), 2088-2092. https://doi.org/10.1039/b315806c.

[154] Zaleśny, R.; Bartkowiak, W.; Styrcz, S.; Leszczynski, J. Solvent Effects on Conformationally Induced Enhancement of the Two-Photon Absorption Cross Section of a Pyridinium-N-Phenolate Betaine Dye. A Quantum Chemical Study. J. Phys. Chem. A 2002, 106 (16), 4032-4037. https://doi.org/10.1021/jp0142684.

[155] Georg, H. C.; Coutinho, K.; Canuto, S. Converged Electronic Polarization of Acetone in Liquid Water and the Role in the $\mathrm{n}-\Pi *$ Transition. Chem. Phys. Lett. 2006, 429 (1-3), 119-123. https://doi.org/10.1016/j.cplett.2006.08.047.

[156] Reichardt, C. Solvatochromic Dyes as Solvent Polarity Indicators. Chem. Rev. 1994, 94 (8), 2319-2358. https://doi.org/10.1021/cr00032a005.

[157] González, D.; Neilands, O.; Caroli Rezende, M. The Solvatochromic Behaviour of 2and 4-Pyridiniophenoxides. J. Chem. Soc. Perkin Trans. 2 1999, No. 30, 713-718. https://doi.org/10.1039/a900470j.

[158] Franco, L. R.; Brandão, I.; Fonseca, T. L.; Georg, H. C. Elucidating the Structure of Merocyanine Dyes with the ASEC-FEG Method. Phenol Blue in Solution. J. Chem. Phys. 2016, 145 (19). https://doi.org/10.1063/1.4967290.

[159] Bistafa, C.; Georg, H. C.; Canuto, S. Combining Ab Initio Multiconfigurational and Free Energy Gradient Methods to Study the $\pi-\Pi^{*}$ Excited State Structure and Properties of Uracil in Water. Comput. Theor. Chem. 2014, 1040-1041, 312-320. https://doi.org/10.1016/j.comptc.2014.04.024.

[160] Lincker, F.; Masson, P.; Nicoud, J.-F.; Didier, P.; Guidoni, L.; Bigot, J.-Y. Synthesis and Characterization of Efficient Two-Photon Absorption Chromophores with Increased Dimensionality. J. Nonlinear Opt. Phys. Mater. 2005, 14 (03), 319-329. https://doi.org/10.1142/S0218863505002761.

[161] Hayek, A.; Bolze, F.; Nicoud, J.-F.; Baldeck, P. L.; Mély, Y. Synthesis and Characterization of Water-Soluble Two-Photon Excited Blue Fluorescent 
Chromophores for Bioimaging. Photochem. Photobiol. Sci. 2006, 5 (1), 102-106. https://doi.org/10.1039/B509843B.

[162] Zerner, M. C. ZINDO, A Semi-Empirical Program Package, University of Florida, Gainsesville, FL 32611, 2000.

[163] Barreto, R. C.; Silva, D. L. PHOTON2: A Program to Calculate the Second Order Hyperpolarizability Using the Sum-Over-States (SOS) Method, 1.0, University of São Paulo, Brazil, 2012.

[164] Van Der Spoel, D.; Lindahl, E.; Hess, B.; Groenhof, G.; Mark, A. E.; Berendsen, H. J. C. GROMACS: Fast, Flexible, and Free. J. Comput. Chem. 2005, 26 (16), 1701-1718. https://doi.org/10.1002/jcc.20291.

[165] Berendsen, H. J. C.; van der Spoel, D.; van Drunen, R. GROMACS: A Message-Passing Parallel Molecular Dynamics Implementation. Comput. Phys. Commun. 1995, 91 (1-3), 43-56. https://doi.org/10.1016/0010-4655(95)00042-E.

[166] Bussi, G.; Donadio, D.; Parrinello, M. Canonical Sampling through Velocity Rescaling. J. Chem. Phys. 2007, 126 (1), 014101. https://doi.org/10.1063/1.2408420.

[167] Parrinello, M.; Rahman, A. Crystal Structure and Pair Potentials: A Molecular-Dynamics Study. Phys. Rev. Lett. 1980, 45 (14), 1196-1199. https://doi.org/10.1103/PhysRevLett.45.1196.

[168] Essmann, U.; Perera, L.; Berkowitz, M. L.; Darden, T.; Lee, H.; Pedersen, L. G. A Smooth Particle Mesh Ewald Method. J. Chem. Phys. 1995, 103 (19), 8577-8593. https://doi.org/10.1063/1.470117.

[169] McDonald, N. A.; Carlson, H. A.; Jorgensen, W. L. Free Energies of Solvation in Chloroform and Water from a Linear Response Approach. J. Phys. Org. Chem. 1997, 10 (7), 563-576. https://doi.org/10.1002/(SICI)1099-1395(199707)10:7<563::AIDPOC912>3.0.CO;2-0.

[170] Frisch, M. J.; Trucks, G. W.; Schlegel, H. B.; Scuseria, G. E.; Robb, M. A.; Cheeseman, J. R.; Scalmani, G.; Barone, V.; Petersson, G. A.; Nakatsuji, H.; et al. Gaussian16 Revision A.03. 2016.

[171] Tessore, F.; Cariati, E.; Cariati, F.; Roberto, D.; Ugo, R.; Mussini, P.; Zuccaccia, C.; Macchioni, A. The Role of Ion Pairs in the Second-Order NLO Response of 4-X-1Methylpiridinium Salts. ChemPhysChem 2010, $11 \quad$ (2), 495-507. https://doi.org/10.1002/cphc.200900696.

[172] Laurent, A. D.; Jacquemin, D. TD-DFT Benchmarks: A Review. Int. J. Quantum Chem. 2013, 113 (17), 2019-2039. https://doi.org/10.1002/qua.24438. 



\section{Anexo A. Multiconfigurational Spectrum Convolution}

Espectros de absorção eletrônica têm sido largamente estudados nas últimas décadas, porém os resultados teóricos geralmente fornecem os valores das energias ressonantes e suas respectivas intensidades. A inclusão de efeitos de alargamento espectral é, então, realizada utilizando funções Gaussianas e Lorentzianas em programas de análises. Atualmente existem diversos softwares capazes de realizar a convolução dos resultados teóricos como o GaussView, Chemcraft, GabEdit, Avogadro entre outros. A maioria deles é limitado a leitura de arquivos de saída de programas de química quântica específicos ou de funções de alargamento, contudo, em nenhum deles é possível obter o espectro teórico considerando um conjunto de configurações como necessário para analisar resultados de cálculos S-QM/MM. Em nosso grupo de pesquisa temos um programa chamado Lorentz que é capaz de convoluir funções Lorentizianas para um conjunto de configurações. O objetivo do Lorentz é obter o espectro de A1F e a densidade de orbitais. Devido à ausência de uma ferramenta apropriada para obter o espectro de $\mathrm{A} 2 \mathrm{~F}$ e a necessidade de obter o espectro com diferentes protocolos (como utilizado no estudo da para-Nitroanilina) decidimos desenvolver nossa própria ferramenta, chamada de Multiconfigurational Spectrum Convolution (MUSPEC).

Contando com uma interface gráfica desenvolvida em Python, é possível analisar os resultados dos espectros de forma interativa e de fácil visualização. Nosso programa, chamado MUSPEC, apresenta como principais características as seguintes opções:

- Opção de leitura de arquivos de saída dos programas Gaussian e Dalton para cálculos de absorção de um e de dois fótons obtidas com métodos TD-DFT.

- Leitura de arquivos no formato "\{estado eletrônico\} \{energia\} \{intensidade\}" para possibilitar a utilização considerando resultados de programas / métodos não implementados.

- Leitura de arquivos com múltiplas colunas para graficar no formato (x,y).

- Convoluções utilizando funções Gaussianas ou Lorentzianas;

- Assumindo $\mathrm{N}$ configurações $\operatorname{com} f$ transições cada, onde $m_{0 f}$ é a probabilidade de transição entre os estados fundamental e $f, \Delta E_{0 f}$ é a energia de transição entre 
os estados fundamental e $f$. A barra superior ${ }^{-}$representa a média sobre as $\mathrm{N}$ configurações e $g(\omega, \Delta E ; \Gamma)$ é a função de alargamento espectral. Os 5 procedimentos são dados por:

○ Procedimento 01 - considerando energia e intensidade média para cada transição e usuário fornece o valor da largura de linha $\Gamma$.

$$
I=\sum_{f} \overline{m_{0 f}} g\left(E, \overline{\Delta E_{0 f}} ; \Gamma\right)
$$

○ Procedimento 02 - o mesmo que o Procedimento 01, porém a largura de linha de cada estado é obtida considerando o desvio padrão da energia, $\Gamma_{\text {std }}=\sqrt{8 \ln 2} \times \sigma_{s t d}\left(\Delta E_{o f}\right)$. Somente aplicável quando mais de uma configuração for selecionada.

$$
I=\sum_{f} \overline{m_{0 f}} g\left(E, \overline{\Delta E_{0 f}} ; \Gamma_{\text {std }}\right)
$$

○ Procedimento 03 - resulta no espectro médio de $\mathrm{N}$ configurações onde o usuário fornece o valor da largura de linha.

$$
I=\frac{1}{N_{\text {conf }}} \sum_{f, N} m_{0 f}^{N} g\left(E, \Delta E_{0 f}^{N} ; \Gamma\right)
$$

○ Procedimento 04 - o mesmo que o Procedimento 03, porém a largura de linha de cada estado é obtida considerando o desvio padrão da energia, $\Gamma_{\text {std }}=\sqrt{8 \ln 2} \times \sigma_{s t d}\left(\Delta E_{o f}\right)$. Somente aplicável quando mais de uma configuração for selecionada.

$$
I=\frac{1}{N_{\text {conf }}} \sum_{f, N} m_{0 f}^{N} g\left(E, \Delta E_{0 f}^{N} ; \Gamma_{\text {std }}\right)
$$

○ Procedimento 05 - resulta no espectro médio de $\mathrm{N}$ configurações onde o usuário fornece o valor da largura de linha total do espectro e o programa ajusta o valor da largura de linha $\Gamma_{\text {fit }}$ de cada configuração.

$$
I=\frac{1}{N_{\text {conf }}} \sum_{f, N} m_{0 f}^{N} g\left(E, \Delta E_{0 f}^{N} ; \Gamma_{\mathrm{fit}}\right)
$$

- Ao definir grupos é possível escolher quais estados serão considerados durante a convolução espectral atribuindo diferentes valores de largura de linha para cada grupo. Junto com o espectro total o usuário pode optar por graficar o espectro de cada grupo e as linhas verticais também separadas por grupos. 
- Fatores de escala estão acessíveis para os dois eixos tão bem como fatores de normalização e de conversão de conversão de energia $\left(\mathrm{eV}, \mathrm{cm}^{-1} \mathrm{e} \mathrm{nm}\right)$.

- Análise dos picos e das larguras completas de cada pico encontrado. Utilizando a rotina find_peaks() implementada na biblioteca SciPy.

- Salvar a imagem em diversos formatos diretamente ou exportar os dados dos espectros para graficar em outros programas.

Abaixo apresentamos algumas figuras que ilustram parte destas características. $\mathrm{Na}$ Figura 38 ilustramos a utilização do programa considerando um único arquivo de saída do programa Gaussian com 197 configurações. Dividimos o espectro em 3 grupos assumindo as transições para os estados $1 ; 2 ; 3$ a 10 com respectivos valores de largura de linha 0.6, 0.35 e $0.2 \mathrm{eV}$. Junto com o espectro total incluímos os espectros obtidos para cada grupo em diferentes cores. Dessa forma é possível observar a contribuição de cada grupo no espectro total. A análise dos picos está apresentada na Figura 39.

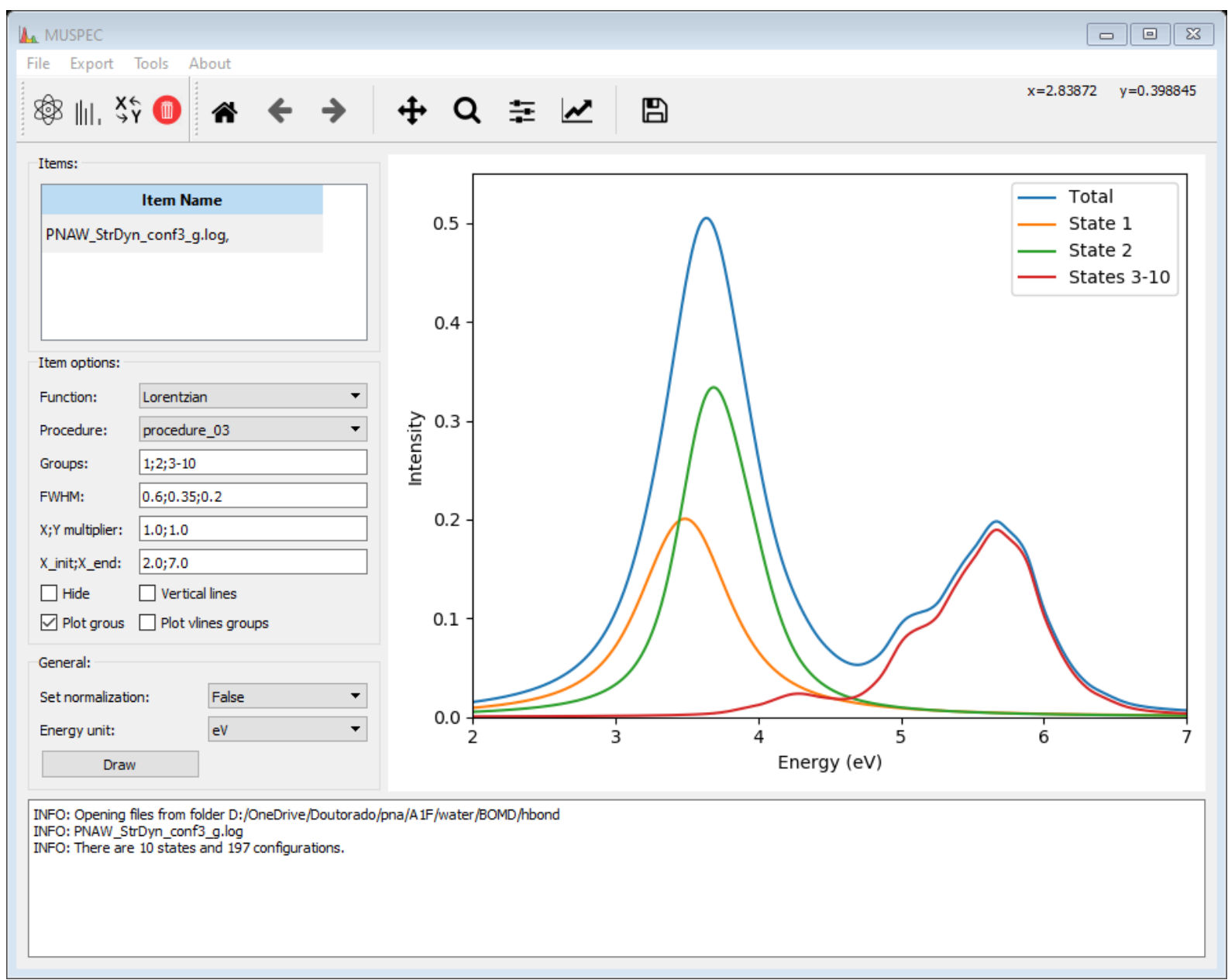

Figura 38. Convolução do espectro de absorção linear considerando funções Lorentzianas para 3 grupos: (1) estado 1 e $\Gamma=$ 0.6; (2) estado 2 e $\Gamma=0.35$; e (3) estados 3 a 10 e $\Gamma=0.2$. 


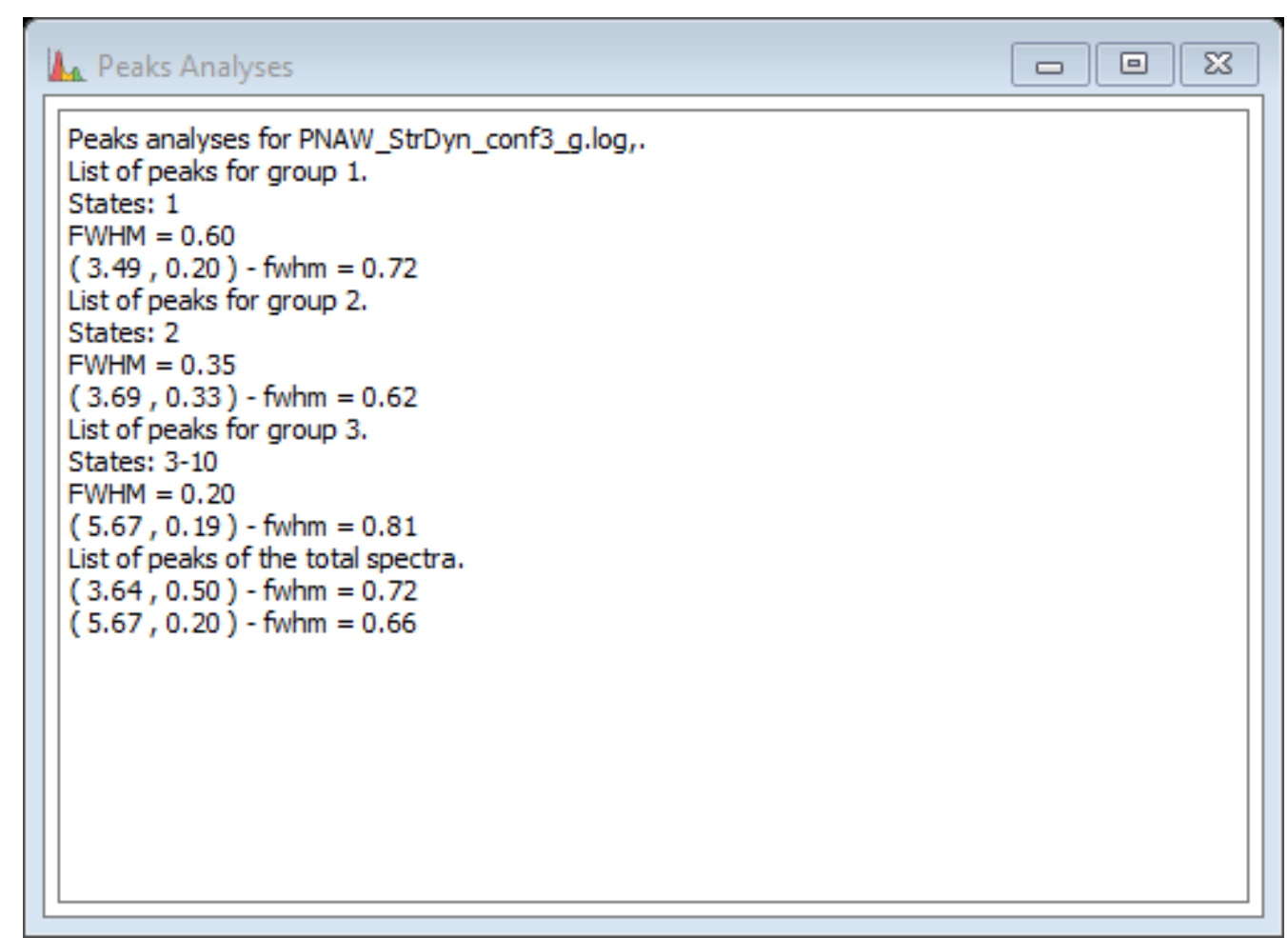

Figura 39. Análise dos picos e larguras de linha do espectro obtidos para cada grupo e para o espectro total referente ao espectro apresentado na Figura 38.

Os espectros obtidos podem ser exportados em arquivos de texto no formato de colunas. A primeira coluna contém os valores de energia e as demais os valores de intensidades para cada grupo e a intensidade do espectro total como pode ser apresentado na Figura 40. A mesma estrutura de colunas será repetida para todos os espectros calculados. 
Tárcius N. Ramos

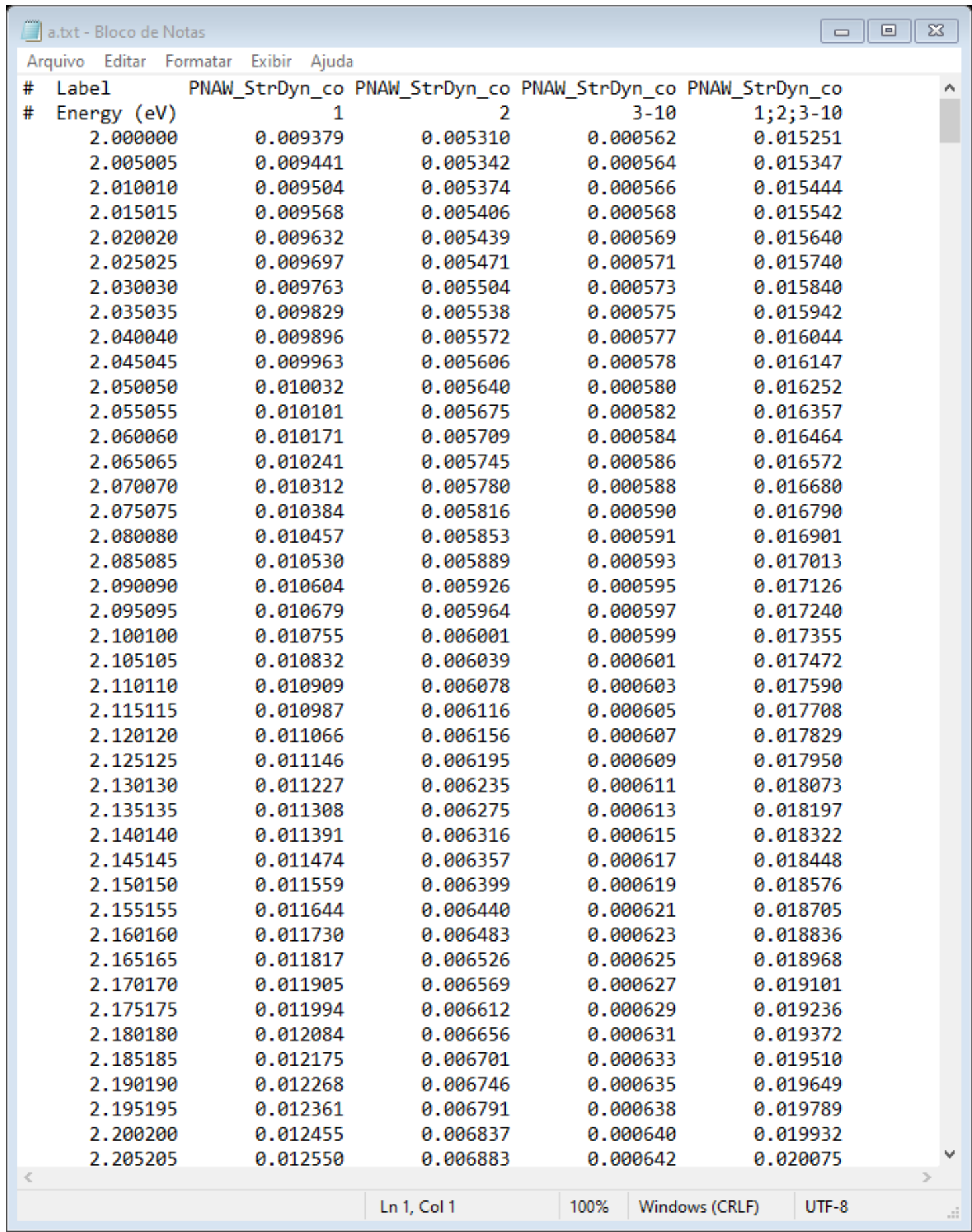

Figura 40. Formato do arquivo de exportação. As colunas são separadas por grupos e o na última coluna as intensidades do espectro total.

O programa está disponibilizado no endereço https://github.com/tarciusnramos/muspec.

Para futuras implementações temos em mente incluir análises de possíveis inversões de transições, aumentar a compatibilidade de leitura de arquivos de saída de programas de química quântica, analisar espectro de distribuição da energia dos orbitais moleculares e deconvoluir espectros experimentais. 


\section{Anexo B. Artigos PUblicados}

Apresentamos a seguir os artigos científicos publicados durante o doutoramento. 


\title{
A theoretical study of the low-lying excited states and the photophysics of dimethoxy curcumin in cyclohexane and acetonitrile
}

\author{
Tárcius N. $\operatorname{Ramos}^{1}\left(\mathbb{D} \cdot\right.$ Sylvio Canuto ${ }^{1}(\mathbb{C}$
}

\begin{abstract}
Two important aspects of the photophysics of dimethoxy curcumin in solvents are theoretically analysed. First, the large Stokes shift in cyclohexane and in acetonitrile is calculated using time-dependent density functional theory with large basis set. Second, a possible explanation is given for the considerable decrease of the nonradiative decay rate in changing from the nonpolar cyclohexane to polar acetonitrile. The solvent is treated using the polarizable continuum model. We analyse different isomers and obtain the absorption transition energies. Similarly, after obtaining the relaxed geometry of the excited state, the emission transition is obtained. In both cases, a red shift of the singlet transition is obtained with increasing solvent polarity. In contrast, the low-lying triplet states are essentially solvent independent. The calculated solvent shift in the emission is in very good agreement with experiment. The Stokes shifts are also in fair agreement with experiment when long-range or dispersion-corrected functionals are used. There are three calculated triplet states lying below the $S_{1}$ state. As they are $\pi \rightarrow \pi^{*}$ states, they cannot participate in the intersystem crossing by the El-Sayed rule. However, a fourth triplet state of $n \rightarrow \pi^{*}$ nature is found only slightly above the Franck-Condon position of the $S_{1}$ state, and the $S_{1} \rightarrow T_{4}$ separation increases with the solvent polarity, this being a possible explanation for the decrease of the nonradiative transition.
\end{abstract}

Keywords Excited state Photophysics - Solvent effects · Stokes shift · Dimethoxy curcumin

Sylvio Canuto canuto@if.usp.br

1 Instituto de Física, Universidade de São Paulo, Cidade Universitária, São Paulo, SP 05508-090, Brazil

\section{Introduction}

Curcumin (CURC) is derived from Curcuma longa, and it has long been used as spice and pigment. It has also been used in a wide range of illness in Asian medicine [1]. Recently, its benign biological properties as antioxidant and anti-inflammatory have been studied [2-8]. The absorption of radiation in the ultraviolet-visible region is related to these biological effects [9]. With the purpose of increasing the biological effects, new compounds have been synthesized and the dimethoxy curcumin (DMC) has been reported to be more promising than CURC [10-12]. The DMC presents two tautomeric forms: the keto-enol form (left picture in Fig. 1) and the diketo form (right picture in Fig. 1). Several interesting studies have been made on the electronic structure of CURC [13-18] and some derivates [19-21] with focus in the tautomerism, deprotonation and photophysics. There are few studies about $\mathrm{DMC}$, and they are focused on ground-state properties and absorptions transition energies [15, 22-24]. There are two interesting aspects of the photophysics of DMC that deserve some attention. First, the emission spectra are characterized by large Stokes shifts, the value being dependent on the solvent used. The second aspect is the large decrease of the nonradiative decay rate when the polarity of the solvent increases. The study of these two aspects is the main subject of the present investigation. In this work, we present a theoretical study of ground and the low-lying excited states of DMC analysing the Stokes shift and the connection between the low-lying singlet and triplet excited states in vacuum and in solvents.

The absorption spectrum of DMC is characterized by strong transition in the region of $410-430 \mathrm{~nm}$ depending on the solvent. In cyclohexane, the transition is located at $410 \mathrm{~nm}(3.02 \mathrm{eV})$, and in acetonitrile, it is red-shifted and 
<smiles></smiles>

Fig. 1 Dimethoxy curcumin tautomers: keto-enol (left) and diketo (right)<smiles>COc1ccc(/C=C/C(=O)CC(=O)/C=C/c2ccc(OC)c(OC)c2)cc1OC</smiles>

located at $419 \mathrm{~nm}(2.96 \mathrm{eV})$. The emission instead appears at $480 \mathrm{~nm}(2.58 \mathrm{eV})$ and $517 \mathrm{~nm}(2.40 \mathrm{eV})$, respectively. Hence, the Stokes shift is $0.44 \mathrm{eV}$ in cyclohexane (nonpolar solvent), while for acetonitrile (polar solvent), it is $0.56 \mathrm{eV}$. This difference in the absorption transition and Stokes shift is a consequence of the different interactions with the solvent. The decrease of the nonradiative decay rate when the polarity of the solvent increases is seen in two specific solvents such as cyclohexane and acetonitrile [23, 24].

Hence, our investigation will consider these two organic solvents: cyclohexane, a nonpolar, and acetonitrile, a polar solvent. In water, both curcumin and dimethoxy curcumin do not fluoresce and these are attributed to an aggregation, forming a nonfluorescent complex [15, 25], and thus are not of interest in this study. Therefore, we will first analyse the possible isomers of DMC, the structure of the ground and singlet excited states, the absorption and emission spectra and, in addition, the low-lying triplet states aiming at a possible understanding of the decrease of the nonradiative decay rate. The use of explicit solvent molecules [26-28] although possible presents an enormous task in this investigation. Several different aspects related to the (1) ground and (2) excited state structures, (3) the absorption and emission transitions, (4) the solvent effects on the Stokes shifts and in the (5) nonradiative transitions are considered. At this stage, some simplification is needed and we will use a continuous representation of the solvent by employing a continuum model $[29,30]$, in particular the polarizable continuum model (PCM) [31, 32] to perform these studies. As we will see, we obtain a qualitative and semiquantitative understanding of the main aspect of the photophysics of DMC.

\section{Computational details}

The geometry optimization of all isomers of DMC was performed using the density functional theory (DFT) method with the three-parameter hybrid functional B3LYP/6-31+G(d,p) [33, 34]. To confirm that the geometries are true minima, the vibrational harmonic frequencies were also calculated. Using these minimum energy structures, the absorption energies and the oscillator strengths were calculated by linear response time-dependent DFT (TD-DFT) [35] using the $6-311++\mathrm{G}(2 \mathrm{~d}, 2 \mathrm{p})$ basis set. In this step, four different hybrid functionals were employed: B3LYP and PBE0 [36] without long-range corrections and CAM-B3LYP [37] and WB97XD [38, 39] that include long-range and dispersion corrections, respectively.

The TD-DFT methodology was also used for the geometry optimizations in the excited state to allow the direct comparison with the ground-state structures. The excited state energies and properties were calculated with the TDDFT in the same calculation level used for obtaining the absorption energies. For inclusion of the solvent effects in excited state properties, the PCM approximation combined with the state-specific methodology [40, 41] was employed. This represents a total relaxation of the solute and the environment. All the calculations were performed with the Gaussian 09 program [42].

The origin of the difference of the nonradiative decay rate in solvent was studied by analysing also triplet states in the solvated condition. The energies of the excited triplet states were also calculated with the Gaussian 09 using TDDFT in the same level of the absorption energies.

\section{Results and discussion}

\subsection{Ground-state geometries}

We first analyse the stability of the possible tautomers. There are four isomers in the keto-enol form and one in the diketo form. Hence, we will consider all these five possibilities. Studies using X-ray indicate that the ketoenol tautomer should be dominant in solution [43]. To obtain the geometries of minimum energy in vacuum, the geometry optimization was carried out using the B3LYP/6-31+G(d,p) level set. The optimized structures are shown in Fig. 2, and the calculated most stable isomer (see below) is shown in evidence (termed D). These geometries are characterized by a planar structure on keto-enol isomers and an out-of-plane structure on diketo form. The structures A, B, C and D differ essentially by the relative position of the $\mathrm{OCH}_{3}$ groups. Structure E corresponds to the diketo form and is largely bent as it is shown in Fig. 2. These final geometries were the starting 
Fig. 2 Optimized geometries of dimethoxy curcumin obtained with B3LYP/6-31+G(d,p) level in vacuum. The calculated more stable isomer (D) is in evidence
A

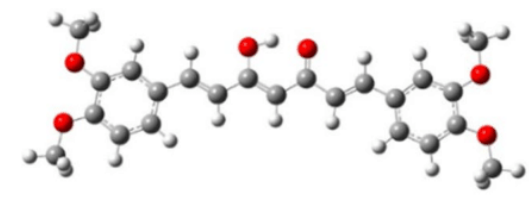

C

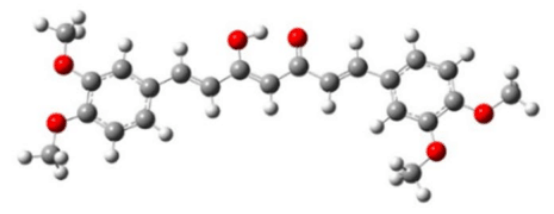

B

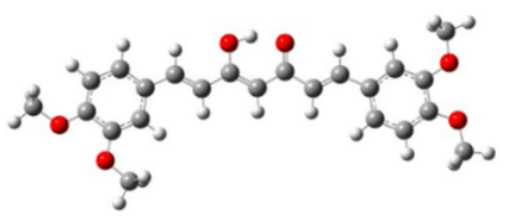

E

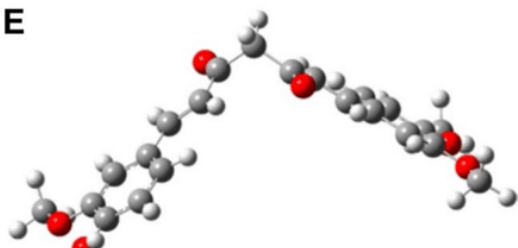

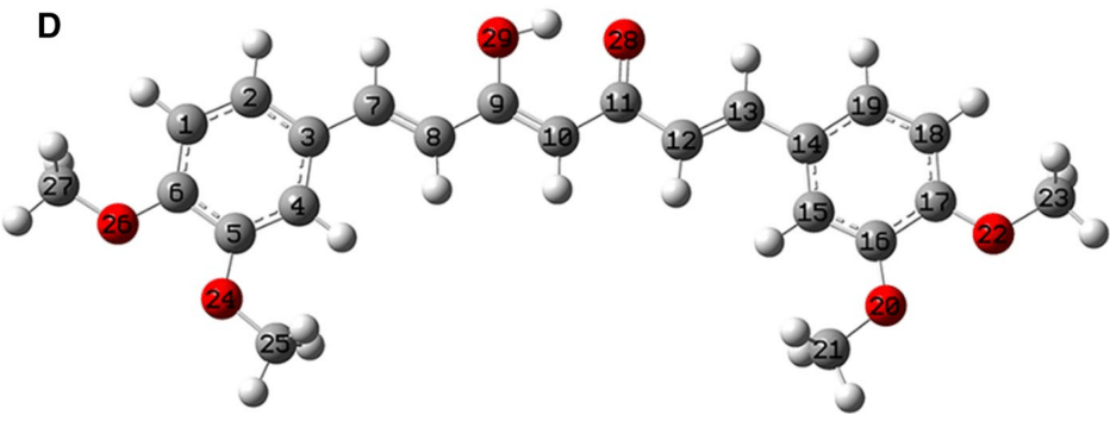

point for the geometry optimization in cyclohexane and acetonitrile environments, with the same calculation level but using the PCM representation of the solvent. Vibration frequency analysis confirms that all structures are true minimum energy. No substantial change was observed comparing the minimum energy structures in different environments. Similarly, for the vibrational modes, these are discernible in essentially three characteristic spectral regions. In the low-frequency side of $\sim 1200 \mathrm{~cm}^{-1}$, we obtain $\mathrm{CH}$ wagging vibrations, around $1630 \mathrm{~cm}^{-1}$ the intense and typical $\mathrm{OH}$ wag and in the high frequency the $\mathrm{OH}$ stretching $\left(\sim 2800 \mathrm{~cm}^{-1}\right)$ and $\mathrm{CH}$ stretches $\left(\sim 3000 \mathrm{~cm}^{-1}\right)$. The internal hydrogen bond present in keto-enol tautomers is a resonant proton transfer between the oxygens, but it is inhibited in protic solvent $[44,45]$. However, due to strong internal interaction and the absence (in cyclohexane) and weak (in acetonitrile) solute-solvent hydrogen interaction, specific solute-solvent interactions were not analysed in this study.

The total free energies were computed using the thermodynamics correction from the harmonic vibrational analysis. In the solvated cases, we also consider the solvation-free energy calculated with the HF/6-31G(d) level set using the united atoms Hartree-Fock cavitation model in PCM. This approach is validated in the study of the solvation-free energy of the emodin molecule in solution where a good agreement is obtained with the results of Monte Carlo simulations [46]. To compare the stability
Table 1 Free energy differences $\Delta \mathrm{G}(\mathrm{kcal} / \mathrm{mol})$ dipole moment $\mu$ (Debye) and relative population $\eta(\%)$ in vacuum, cyclohexane and acetonitrile

\begin{tabular}{|c|c|c|c|c|c|c|c|c|c|}
\hline & \multicolumn{3}{|c|}{ Vacuum } & \multicolumn{3}{|c|}{ Cyclohexane } & \multicolumn{3}{|c|}{ Acetonitrile } \\
\hline & $\Delta \mathrm{G}$ & $\mu$ & $\eta$ & $\Delta \mathrm{G}$ & $\mu$ & $\eta$ & $\Delta \mathrm{G}$ & $\mu$ & $\eta$ \\
\hline A & 0.94 & 3.92 & 10 & 0.42 & 4.60 & 17 & 0.45 & 5.91 & 16 \\
\hline B & 0.42 & 3.75 & 23 & 0.12 & 4.34 & 28 & 0.14 & 5.38 & 26 \\
\hline $\mathrm{C}$ & 0.50 & 3.65 & 20 & 0.31 & 4.21 & 20 & 0.21 & 5.23 & 24 \\
\hline D & 0.00 & 3.45 & 47 & 0.00 & 3.91 & 35 & 0.00 & 4.65 & 34 \\
\hline E & 10.57 & 2.80 & 0 & 8.08 & 3.33 & 0 & 8.01 & 3.38 & 0 \\
\hline
\end{tabular}

For $\Delta \mathrm{G}$ in solution, part of the results were calculated by $\mathrm{HF} / 6-31 \mathrm{G}(\mathrm{d})$ and all other results were calculated with B3LYP/6-31+G(d,p) 
of the isomers, we consider the total free energy variation $(\Delta \mathrm{G})$ between the isomer and the most stable compound (Table 1). The solvent effects in the ground state reduce by half the $\Delta \mathrm{G}$ of keto-enol isomers when compared with vacuum results. The diketo isomer has $\Delta \mathrm{G}$ decreased from $10.57 \mathrm{kcal} / \mathrm{mol}$ in vacuum to 8.08 and $8.01 \mathrm{kcal} /$ mol in cyclohexane and in acetonitrile, respectively. We also compute the relative population of the isomers using the Boltzmann distribution $\left(\eta_{i}=e^{-\Delta G_{i} / k T} / \sum_{i} e^{-\Delta G_{i} / k T}\right)$, where $k$ and $T$ are, respectively, the Boltzmann constant and the temperature. The relative population was calculated at the room temperature $(298.15 \mathrm{~K})$. Our results show that the isomer D is the most stable in all conditions considered. The isomer $\mathrm{B}$ is the second more stable with a difference of $0.42 \mathrm{kcal} / \mathrm{mol}$ in vacuum, 0.12 and $0.14 \mathrm{kcal} / \mathrm{mol}$ in cyclohexane and in acetonitrile, respectively. To specify better the relative energies between the isomers B and D, we have calculated the rotational energy barrier between these two isomers both in vacuum and in acetonitrile. Using the B3LYP/6-31+G(d,p), we obtain the barriers of 6.4 and $7.3 \mathrm{kcal} / \mathrm{mol}$, respectively, in vacuum and acetonitrile. Thus, although the relative minimum energy between the isomers $\mathrm{B}$ and $\mathrm{D}$ is decreased in the solvent, the barrier for interconversion increases instead. But the value of these energy barriers and the relative population $(\eta)$, shown in Table 1 , indicates that isomers A, B, C and D can coexist and justify the structures observed by Galer et al. [43]. The relative free energy variation $(\Delta \mathrm{G})$, dipole moment $(\mu)$ and population data $(\eta)$ of these isomers are presented in Table 1. These results corroborate the experimental finding that the keto-enol form is dominant in solution and that the isomer $\mathrm{D}$ is found to be the most stable

\subsection{Absorption spectra}

To analyse the low-lying excited states, the first six singlet and triplet electronic excited states for all isomers were calculated using the TD-DFT method in the corresponding optimized structures. For all keto-enol isomers, the first singlet excited state $\left(S_{1}\right)$ carries the intensity that characterizes the first observed band. In the diketo form, instead, the $S_{2}$ is the more intense when in vacuum. In solution, all isomers absorb for the $S_{1}$ state that is well described by the HOMO-LUMO transition. In fact, all these low-lying transitions are $\pi \rightarrow \pi^{*}$ except one $n \rightarrow \pi^{*}$, the HOMO-5 that is calculated as the $S_{2}$ or $S_{3}$ depending on the functional. The Kohn-Sham molecular orbitals are similar for vacuum, cyclohexane and acetonitrile and are shown in Fig. 3. The $S_{1}$ state is a $\pi \rightarrow \pi *$ transition and thus expected to exhibit a red shift upon solvation.

Considering the transition energies of all isomers, the absorption spectrum can be obtained using the results for the individual isomers weighted by $\eta$. A comparison of this with the spectrum obtained using only the most stable isomer $\mathrm{D}$ gives the same description of the absorption process in all solvents studied. Therefore, in discussing the results, we consider only the isomer $\mathrm{D}$.

In Table 2 are presented the absorption transition energies and the corresponding oscillator strengths for isomer D. As discussed above, we find a $n \rightarrow \pi^{*}$ among the first three excitations and the position changes depending on the functional adopted. We focus on the CAM-B3LYP model and note that this $n \rightarrow \pi^{*}$ transition corresponds to the $S_{2}$ state in vacuum but the $S_{3}$ in both solvents. This interchange is due to the red shift of the $S_{3}$ state, as expected for a $\pi \rightarrow \pi^{*}$ states. The $n \rightarrow \pi^{*}$ state, instead, as expected,
Fig. 3 Kohn-Sham molecular orbitals in vacuum and in acetonitrile calculated using CAM-B3LYP/6-311++G(d,p)

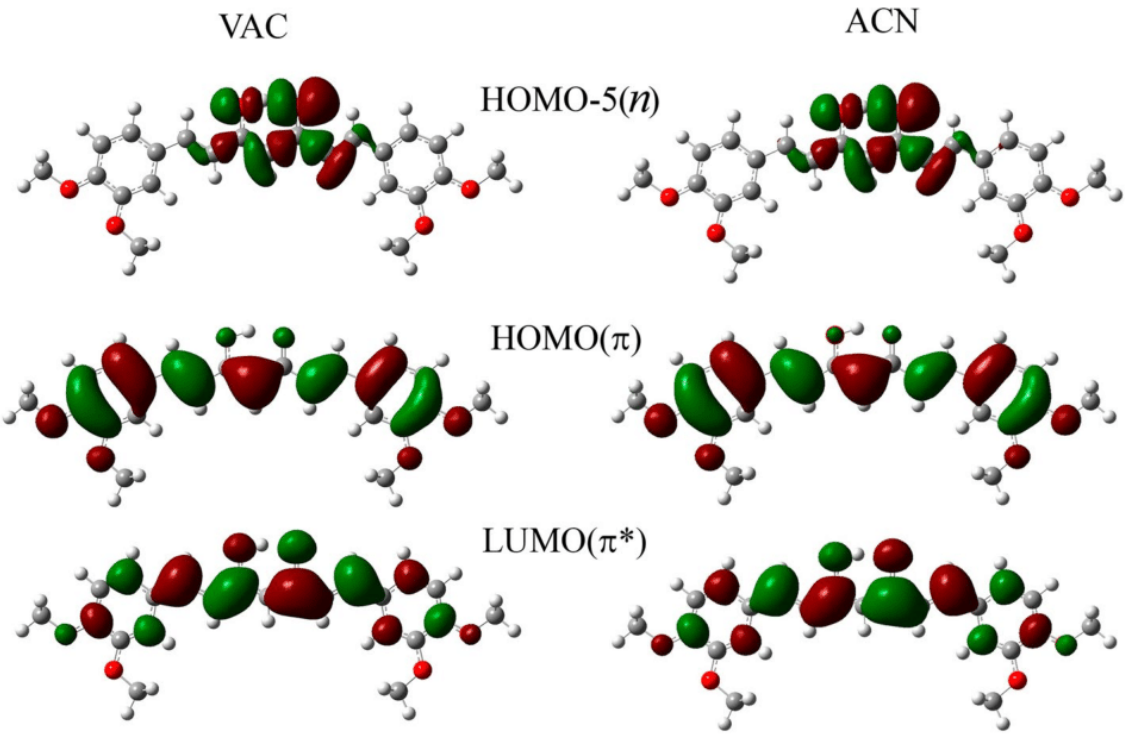


Table 2 Absorption energies of the isomer $\mathrm{D}$ in vacuum, cyclohexane and acetonitrile

\begin{tabular}{|c|c|c|c|c|c|c|c|}
\hline \multirow[t]{2}{*}{ Functional } & \multirow[t]{2}{*}{ Transition } & \multicolumn{2}{|c|}{ Vacuum } & \multicolumn{2}{|c|}{ Cyclohexane } & \multicolumn{2}{|c|}{ Acetonitrile } \\
\hline & & $\Delta \mathrm{E}_{\mathrm{abs}}$ & $f$ & $\Delta \mathrm{E}_{\mathrm{abs}}$ & $f$ & $\Delta \mathrm{E}_{\mathrm{abs}}$ & $f$ \\
\hline \multirow[t]{3}{*}{ B3LYP } & $S_{1}$ & 2.85 & 1.629 & 2.69 & 1.813 & 2.62 & 1.797 \\
\hline & $S_{2}$ & 3.21 & 0.019 & 3.10 & 0.027 & 3.02 & 0.029 \\
\hline & $S_{3}$ & 3.41 & $0.0^{+}$ & 3.46 & $0.0^{+}$ & 3.49 & 0.140 \\
\hline \multirow[t]{3}{*}{ PBE0 } & $S_{1}$ & 2.95 & 1.738 & 2.79 & 1.914 & 2.72 & 1.899 \\
\hline & $S_{2}$ & 3.37 & 0.022 & 3.25 & 0.032 & 3.17 & 0.034 \\
\hline & $S_{3}$ & 3.50 & $0.0^{+}$ & 3.55 & $0.0^{+}$ & 3.62 & $0.0^{+}$ \\
\hline \multirow[t]{3}{*}{ CAM-B3LYP } & $S_{1}$ & 3.30 & 2.043 & 3.13 & 2.196 & 3.07 & 2.194 \\
\hline & $S_{2}$ & 3.85 & $0.0^{+}$ & 3.79 & 0.068 & 3.75 & 0.070 \\
\hline & $S_{3}$ & 3.96 & 0.055 & 3.89 & $0.0^{+}$ & 3.95 & $0.0^{+}$ \\
\hline \multirow[t]{3}{*}{ WB97XD } & $S_{1}$ & 3.36 & 2.077 & 3.19 & 2.229 & 3.14 & 2.231 \\
\hline & $S_{2}$ & 3.81 & $0.0^{+}$ & 3.85 & $0.0^{+}$ & 3.85 & 0.074 \\
\hline & $S_{3}$ & 4.05 & 0.060 & 3.88 & 0.073 & 3.91 & $0.0^{+}$ \\
\hline Experimental & & - & - & 3.02 & - & 2.96 & - \\
\hline
\end{tabular}

The transition energy values are given in $\mathrm{eV}$ and $f$ is the dimensionless oscillator strength. Experimental results are from references $[23,24]$

+ Indicates the $n \rightarrow \pi *$ transition

suffers a blue shift upon solvation. As the $n \rightarrow \pi^{*}$ carries no intensity, the spectrum is dominated by the lowest excited state calculated in vacuum at $3.30 \mathrm{eV}$. In cyclohexane and in acetonitrile, the $S_{1}$ state is obtained at $3.13 \mathrm{eV}$ and $3.07 \mathrm{eV}$, respectively, only $0.1 \mathrm{eV}$ above experiment $[23,24]$. The corresponding calculated bathochromic shift of this $\mathrm{S}_{1}$ state is $0.17 \mathrm{eV}$ in cyclohexane and $0.23 \mathrm{eV}$ in acetonitrile. This is in line with experiment. The calculated solvatochromic shift from cyclohexane to acetonitrile is $0.06 \mathrm{eV}$ in sharp agreement with experiment [23, 24].

Comparing the theoretical and experimental maximum absorption values, the CAM-B3LYP shows the best agreement with an error of $0.11 \mathrm{eV}$ both for cyclohexane and for acetonitrile. In red solid lines of Fig. 4a are shown the energy values for the $\pi \rightarrow \pi^{*}$ singlet states, while the red dashed line indicates the singlet $n \rightarrow \pi^{*}$ transitions.
For subsequent analysis, we also consider the excited triplet states: the $\pi \rightarrow \pi^{*}$ (blue dotted-dashed lines) and the $n \rightarrow \pi^{*}$ (blue dotted lines). There are three $\pi \rightarrow \pi^{*}$ triplet states below $S_{1}$. These states are calculated to be solvent independent, but it can note a pronounced red shift of the $S_{1}$ state.

\subsection{Excited states geometries}

For the excited state, only the isomer D was investigated. The geometries in vacuum and in solution were optimized in the $S_{1}$ excited state using the TD-B3LYP/6-31+G(d,p) calculation level. The vibrational analysis was made to confirm the nature of the minimum energy. Comparing the ground and excited state structures in vacuum, we can observe a torsion of $12^{\circ}$ around the $\mathrm{C} 13-\mathrm{C} 14$ bond (see
Fig. 4 Excited state energies for singlets (red lines) $\pi \rightarrow \pi^{*}$ solid lines, $n \rightarrow \pi *$ dashed lines, and for triplets (blue lines): $\pi \rightarrow \pi^{*}$ dotted-dashed lines, $n \rightarrow \pi^{*}$ dotted lines. In panel (a), the excited state energies calculated in ground-state minimum structures; in (b), the excited state energies calculated in $S_{1}$ geometry. All results calculated with CAM-B3LYP/6$311++\mathrm{G}(2 \mathrm{~d}, 2 \mathrm{p})$

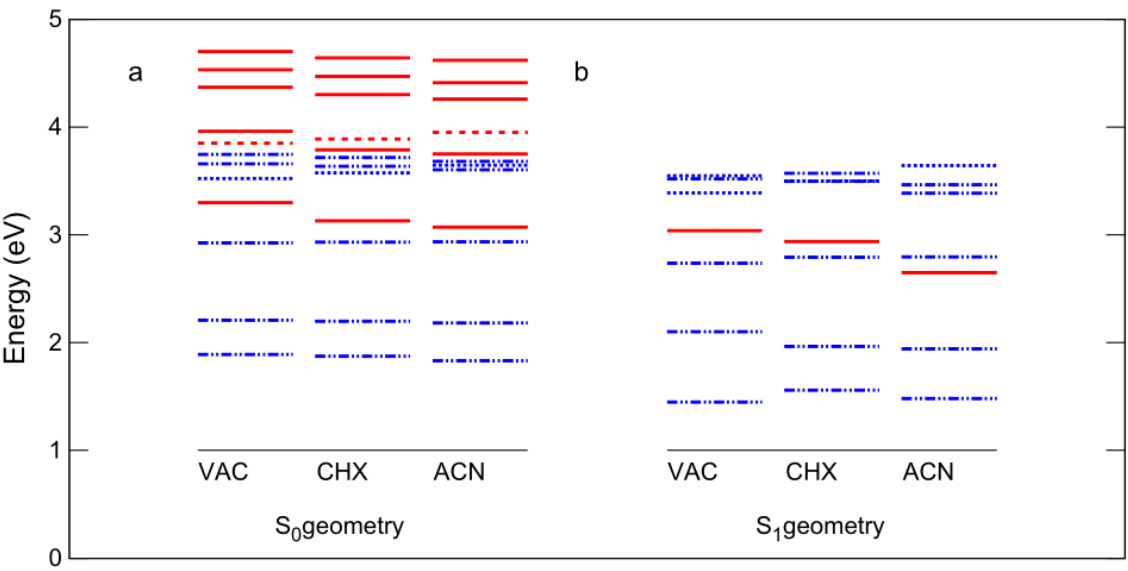


atomic labels in Fig. 2). The bonds in central chain (C3-RC12) increase by $0.05 \AA$, and the intramolecular hydrogen bond increases from 1.553 to $1.588 \AA$. For cyclohexane and for acetonitrile (in parenthesis), the bond distances in chain C3-R-C14 increase until 0.027 (0.029) $\AA$ and the intramolecular hydrogen bond decreases from 1.550 (1.546) $\AA$ to 1.527 (1.527). The torsional angles in solvents are no larger than $1.5^{\circ}$.

The in-vacuum dipole moment of the $S_{1}$ state in the Franck-Condon region is $5.03 \mathrm{D}$ and thus $1.76 \mathrm{D}$ larger than in the ground state (Table 3). We use the notation where $\vec{\mu}\left(S_{x}, S_{y}\right)$ represents the dipole moment of the electronic state $S_{y}$ calculated in the geometry of the $S_{x}$ state. This increase in the dipole moment explains the red shift upon solvation. In acetonitrile, this dipole increase is 3.25 D. Barik and Priyadarsini [23] analysed their experimental results in the framework of the Lippert-Mataga equation to estimate an excited state dipole moment increased by ca. $4.9 \mathrm{D}$ compared to the ground state. A comparison of the variation of the dipole moment vector upon excited state relaxation is also interesting, and these are shown in Fig. 5. After excitation, the dipole moment of the $S_{1}$ state in the Franck-Condon region has essentially the same direction as in the fully relaxed conformation in solution, with a small difference in the in-vacuum case. This slight change originates in the torsion of $12^{\circ}$ around the
C13-C14 bond, described above. However, the absolute value changes and this is best obtained by the vectorial difference $\mu_{x}^{y}=\left|\vec{\mu}\left(S_{x}, S_{y}\right)-\vec{\mu}\left(S_{x}, S_{x}\right)\right|$ that gives information about the charge rearrangement upon transition. These are also given in Table 3. In vacuum and in acetonitrile, we observe more charge transfer during the emission process $\left(\mu_{0}^{1}<\mu_{1}^{0}\right)$. However, in cyclohexane, there is more charge transfer during the absorption than the emission $\left(\mu_{0}^{1}>\mu_{1}^{0}\right)$.

\subsection{Emission transitions}

\subsubsection{The Stokes shift}

The energies of the vertical emission transitions $S_{1} \rightarrow S_{0}$ were calculated in the solvated cases using the state-specific methodology [40, 41]. All these calculations were made in the optimized geometry of the $S_{1}$ state, and the optimizations were made in vacuum and in solvent. We thus assume that the lifetime of the excited state is large enough to allow for the structural relaxation as indeed suggested by experiment [23, 24]. But, of course, the emission may begin before the full geometry relaxation. Table 4 shows the calculated results. We focus again on the results obtained using CAM-B3LYP/6-311++G(2d,2p). The emission in vacuum is calculated as $3.04 \mathrm{eV}$ with a Stokes shift of $0.26 \mathrm{eV}$. In cyclohexane, the emission is
Table 3 Ground and excited state dipole moments calculated in different electronic and structural conditions and the difference in a vertical transition, calculated with CAM-B3LYP/6 $311++\mathrm{G}(2 \mathrm{~d}, 2 \mathrm{p})$

Fig. 5 Orientation of the calculated dipole moment in the ground state (top), first excited state in the Franck-Condon geometry (middle) and fully relaxed first excited state for vacuum and acetonitrile

\begin{tabular}{llllllll}
\hline & $\mu\left(S_{0}, S_{0}\right)$ & $\mu\left(S_{0}, S_{1}\right)$ & $\mu\left(S_{1}, S_{1}\right)$ & $\mu\left(S_{1}, S_{0}\right)$ & $\mu_{0}^{1}$ & $\mu_{1}^{0}$ & $\left|\vec{\mu}^{e}-\vec{\mu}^{g}\right|$ \\
\hline VAC & 3.27 & 5.03 & 5.03 & 4.42 & 2.19 & 3.03 & 1.95 \\
CHX & 3.69 & 5.95 & 6.59 & 4.43 & 2.77 & 2.54 & 3.28 \\
ACN & 4.34 & 7.59 & 9.54 & 6.22 & 3.90 & 4.03 & 6.01 \\
\hline
\end{tabular}

$\vec{\mu}^{e}=\vec{\mu}\left(S_{0}, S_{0}\right)$ and $\vec{\mu}^{g}=\vec{\mu}\left(S_{1}, S_{1}\right)$ Values in Debye

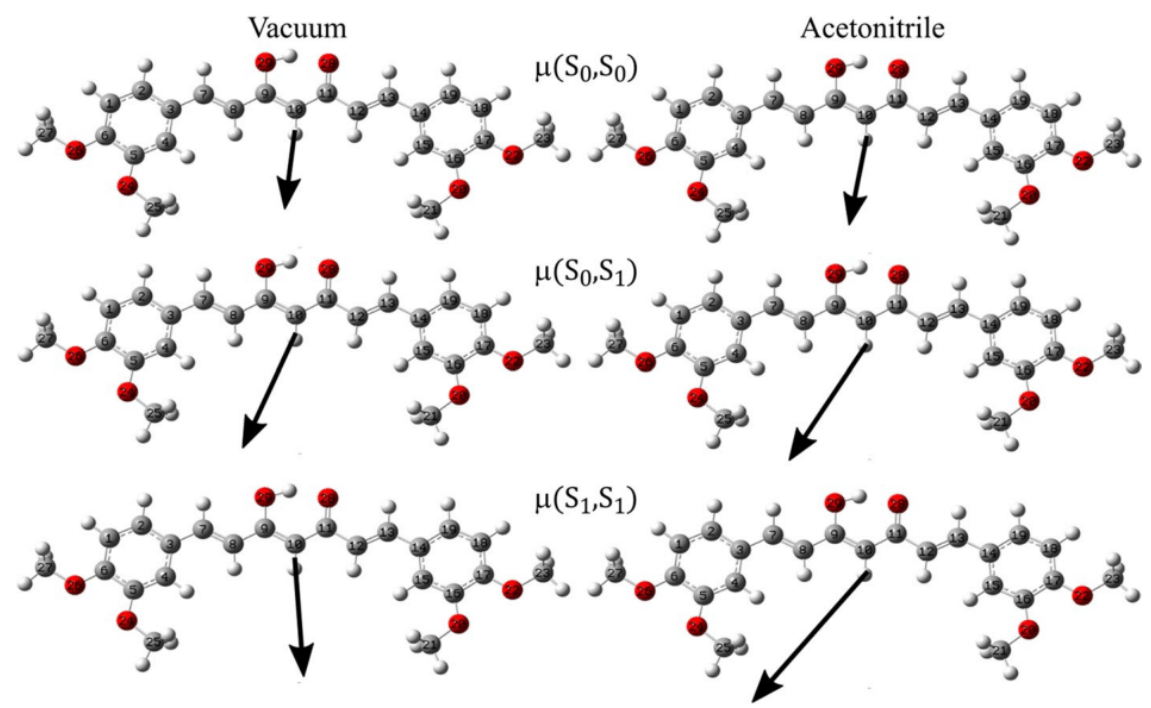


Table 4 Transition energy from the $S_{1}$ to the ground state and the calculated Stokes shift for the isomer D in vacuum, in cyclohexane and in acetonitrile

\begin{tabular}{|c|c|c|c|c|c|c|c|}
\hline \multirow[t]{2}{*}{ Functional } & \multicolumn{2}{|c|}{ Vacuum } & \multicolumn{2}{|l|}{ Cyclohexane } & \multicolumn{2}{|l|}{ Acetonitrile } & \multirow[t]{2}{*}{$\Delta \mathrm{E}_{\mathrm{CHX}}-\Delta \mathrm{E}_{\mathrm{ACN}}$} \\
\hline & $\Delta \mathrm{E}$ & Stokes Shift & $\Delta \mathrm{E}$ & Stokes shift & $\Delta \mathrm{E}$ & Stokes shift & \\
\hline B3LYP & 2.50 & 0.35 & 2.51 & 0.18 & 1.91 & 0.71 & 0.60 \\
\hline PBE0 & 2.63 & 0.32 & 2.65 & 0.14 & 2.11 & 0.61 & 0.54 \\
\hline CAM-B3LYP & 3.04 & 0.26 & 2.94 & 0.19 & 2.65 & 0.42 & 0.29 \\
\hline WB97XD & 3.10 & 0.26 & 3.00 & 0.19 & 2.73 & 0.41 & 0.27 \\
\hline Experimental & - & - & $2.59(2.62)$ & $0.44(0.40)$ & $2.40(2.38)$ & $0.56(0.58)$ & $0.19(0.24)$ \\
\hline
\end{tabular}

The transition energy values are in eV. Experimental results are from Ref. [23] and in parenthesis Ref. [24] red-shifted by $0.1 \mathrm{eV}$ and is obtained at $2.94 \mathrm{eV}$. This gives a Stokes shift of $0.19 \mathrm{eV}$, less than the experimental value of $0.40-0.43 \mathrm{eV}$. We note that the origin of this discrepancy is the large value of the emission energy at $2.94 \mathrm{eV}$ compared with the experiment at $2.59-2.62 \mathrm{eV}$. The result for acetonitrile is slightly better with an emission at $2.65 \mathrm{eV}$, compared with experiment at $2.38-2.40 \mathrm{eV}$. This leads to a Stokes shift of $0.42 \mathrm{eV}$ in better agreement with the experimental value of $0.56-0.58 \mathrm{eV}$. The solvent effect on the emission transition, $\Delta \mathrm{E}_{\mathrm{CHX}}-\Delta \mathrm{E}_{\mathrm{ACN}}$, is, however, calculated with the CAM-B3LYP/6-311++G(2d,2p) model in reasonably good agreement with experiment. One may note that the B3LYP and PBE0 present results that are of lower quality compared to the CAM-B3LYP and WB97XD with long-range and dispersion corrections. In general, the Stokes shift is better obtained for acetonitrile and the solvent shift for the emission is well described.

\subsubsection{Nonradiative decay}

We finally analyse possible reasons for the decrease in the nonradiative decay rate in changing from cyclohexane to acetonitrile. An important ingredient is the solvent effects on the triplet states leading to a possible intersystem crossing process [23, 24, 47]. It has been noted before, in the case of curcumin, that the $S_{1}$ state is red-shifted with increased solvent polarity, whereas the $T_{1}$ state is essentially solvent independent [47]. With this, it has been suggested [23] that in the related DMC, the energy barrier to cross from the singlet to the triplet is increased, thus decreasing the rate of intersystem crossing. We now consider the calculated triplet states of DMC, shown in Fig. 4a. The relative locations of the lowest singlet and triplet states calculated at the Franck-Condon region of the absorption (i.e., in the $\mathrm{S}_{0}$ equilibrium geometry) are shown. It can be seen that the $S_{1}$ state is close to $T_{3}$, not $T_{1}$. But all the three lowest triplet states are essentially insensitive to solvent. In vacuum, the $S_{1}$ state lies above the $T_{3}$ state with a separation that is $0.38 \mathrm{eV}$. But the closest $n \rightarrow \pi^{*}$ state to $S_{1}$ is $T_{4}$, located $0.22 \mathrm{eV}$ above.

Table 5 presents the energies of the lowest four triplet states calculated in the $S_{0}$ Franck-Condon region.
Table 5 Calculated lowest triplet states $(\mathrm{eV})$ of DMC in the FranckCondon region of the $S_{0}$ state in vacuum, cyclohexane and acetonitrile using CAM-B3LYP/6-311 + $\mathrm{G}(2 \mathrm{~d}, 2 \mathrm{p})$

\begin{tabular}{llll}
\hline State & Vacuum & Cyclohexane & Acetonitrile \\
\hline$S_{1}\left(\pi \rightarrow \pi^{*}\right)$ & 3.30 & 3.13 & 3.07 \\
$T_{1}\left(\pi \rightarrow \pi^{*}\right)$ & 1.89 & 1.87 & 1.83 \\
$T_{2}\left(\pi \rightarrow \pi^{*}\right)$ & 2.21 & 2.20 & 2.18 \\
$T_{3}\left(\pi \rightarrow \pi^{*}\right)$ & 2.92 & 2.93 & 2.94 \\
$T_{4}\left(n \rightarrow \pi^{*}\right)$ & 3.52 & 3.57 & $3.60\left(\pi \rightarrow \pi^{*}\right)$ \\
& & & $3.66\left(n \rightarrow \pi^{*}\right)$ \\
\hline
\end{tabular}

For comparison, the in-vacuum $\mathrm{T}_{1}$ result for curcumin is $1.95 \mathrm{eV}$ [47]

For triplet states obtained within the PCM representation of the solvent, it may also be convenient to perform state-specific calculation. These were also performed and will be discussed after presenting the results with linear response given in Table 5. It can be seen that the three triplet states lying lower than the first excited singlet state $S_{1}$ are all of $\pi \rightarrow \pi^{*}$ nature. By the El-Sayed rule, the intersystem crossing between $S_{1}$ and these triplet states is forbidden. The lowest triplet $n \rightarrow \pi^{*}$ state is $T_{4}$ that is close but slightly above $S_{1}$. The energy difference in vacuum is $0.22 \mathrm{eV}$ allowing for vibrational overlap. Thus, this is the natural candidate for the intersystem crossing and to originate the nonradiative transition. Whereas $T_{1}-T_{3}$ are insensitive to the solvent, the $T_{4}$ state is blue-shifted showing an increased singlet-triplet $S_{1} \rightarrow T_{4}$ separation. In acetonitrile, a fourth $\pi \rightarrow \pi^{*}$ state appears and the lowest $n \rightarrow \pi^{*}$ state becomes $T_{5}$. Hence, it is expected that by increasing the solvent polarity, there is a decrease in the nonradiative transition by the difficult in reaching the $n \rightarrow \pi^{*}$ triplet from the $S_{1}$ state. Figure $4 \mathrm{~b}$ shows the relative position of the lowest singlet and triplet state from the relaxed geometry of the $S_{1}$ state. Now, in acetonitrile there is a reversal where now the $S_{1}$ state lies lower than the $T_{3}$ state, but again these are $\pi \rightarrow \pi^{*}$ states. Considering now the results obtained with state-specific calculations, the numerical changes compared to the linear response are small and in some cases only minor. $\mathrm{T}_{1}, \mathrm{~T}_{2}$ and $\mathrm{T}_{3}$ are essentially unaltered. The triplet $n \rightarrow \pi^{*}$ state that is to take part in the 
photophysics is calculated essentially the same in cyclohexane but slightly increases the separation with respect to the $S_{1}$ state in the case of acetonitrile (calculated now at $3.72 \mathrm{eV}$ ), thus favouring more the argument already used from the linear response results. The picture is qualitatively the same, with the increase of the separation between the $S_{1}$ and the $n \rightarrow \pi^{*}$ triplet state. This seems to give a possible explanation for the increased fluorescence observed with the increased solvent polarity.

\section{Conclusions}

The electronic structure of dimethoxy curcumin was theoretically studied in vacuum and in two solvents: cyclohexane and acetonitrile. The keto-enol tautomer was found to be more stable than the diketo form as expected by previous studies. Despite having different isomers, the sole use of the most stable isomer reproduces well the absorption spectrum. The absorption is characterized by an increase in the dipole moment leading to an expected red shift of the $S_{0} \rightarrow S_{1}\left(\pi-\pi^{*}\right)$ transition. The calculated solvation shifts both in the absorption and in emission are well described. The calculated Stokes shifts are also in fair agreement with experimental values for acetonitrile but less accurate for cyclohexane. Possible improvements can be obtained by the explicit use of solvent molecules. Four different density functionals are used in the calculations, and systematically better agreement is obtained with those that include long-range (CAM-B3LYP) and dispersion correction (WB97XD). Three excited triplet states are found to be lower than the intense $S_{1}$ state, and these are essentially solvent independent as opposed to the $S_{1}$ state that is redshifted. All these states are of $\pi \rightarrow \pi^{*}$ nature, and intersystem crossing is forbidden by the El-Sayed rule. A fourth triplet state of $n \rightarrow \pi^{*}$ nature is suggested to be responsible by the intersystem crossing from the $S_{1}$ state. By increasing the solvent polarity, this $n \rightarrow \pi^{*}$ state separates more from the $S_{1}$ state decreasing the singlet-triplet intersystem transition, hence decreasing the nonradiative emission. This characteristic is one of our contentions for the increase in the fluorescence rate of dimethoxy curcumin with increased solvent polarity.

Acknowledgements We thank Dr. Y. Orozco-Gonzales and Dr. Marcelo H. Cardenuto for discussions. This work is partially supported by grant 2013/01,871-5 from São Paulo Research Foundation (FAPESP), CNPq, CAPES and INCT-FCx.

\section{References}

1. Benzie I (2011) Herbal medicine: biomolecular and clinical aspects. CRC Press, Boca Raton
2. Sharma OP (1976) Antioxidant activity of curcumin and related compounds. Biochem Pharmacol 25:1811-1812. doi:10.1016/0006-2952(76)90421-4

3. Khopde M, Priyadarsini S, Venkatesan KI, Rao MNA P (1999) Free radical scavenging ability and antioxidant efficiency of curcumin and its substituted analogue. Biophys Chem 80:85-91. doi:10.1016/S0301-4622(99)00070-8

4. Banerjee A, Kunwar A, Mishra B, Priyadarsini KI (2008) Concentration dependent antioxidant/pro-oxidant activity of curcumin. Chem Biol Interact 174:134-139. doi:10.1016/j. cbi.2008.05.009

5. Sun Y-M, Zhang H-Y, Chen D-Z, Liu C-B (2002) Theoretical elucidation on the antioxidant mechanism of curcumin: a DFT Study. Org Lett 4:2909-2911. doi:10.1021/ol0262789

6. Chignell CF, Bilskj P, Reszka KJ et al (1994) Spectral and photochemical properties of curcumin. Photochem Photobiol 59:295302. doi:10.1111/j.1751-1097.1994.tb05037.x

7. Goel A, Kunnumakkara AB, Aggarwal BB (2008) Curcumin as "Curecumin": from kitchen to clinic. Biochem Pharmacol 75:787-809. doi:10.1016/j.bcp.2007.08.016

8. Balasubramanian K (2006) Molecular orbital basis for yellow curry spice curcumin's prevention of Alzheimer's disease. J Agric Food Chem 54:3512-3520. doi:10.1021/jf0603533

9. Hegge AB, Bruzell E, Kristensen S, Tønnesen HH (2012) Photoinactivation of Staphylococcus epidermidis biofilms and suspensions by the hydrophobic photosensitizer curcumin Effect of selected nanocarrier: studies on curcumin and curcuminoides XLVII. Eur J Pharm Sci 47:65-74. doi:10.1016/j. ejps.2012.05.002

10. Ohtsu H, Xiao Z, Ishida J et al (2002) Antitumor agents. 217. curcumin analogues as novel androgen receptor antagonists with potential as anti-prostate cancer agents. J Med Chem 45:50375042. doi: $10.1021 / \mathrm{jm} 020200 \mathrm{~g}$

11. Lin L, Shi Q, Nyarko AK et al (2006) Antitumor agents. 250. Design and synthesis of new curcumin analogues as potential anti-prostate cancer agents. J Med Chem 49:3963-3972. doi:10.1021/jm051043z

12. Amolins MW, Peterson LB, Blagg BSJ (2009) Synthesis and evaluation of electron-rich curcumin analogues. Bioorg Med Chem 17:360-367. doi:10.1016/j.bmc.2008.10.057

13. Benassi R, Ferrari E, Lazzari S et al (2008) Theoretical study on Curcumin: a comparison of calculated spectroscopic properties with NMR, UV-vis and IR experimental data. J Mol Struct 892:168-176. doi:10.1016/j.molstruc.2008.05.024

14. Shen L, Ji H-F (2007) Theoretical study on physicochemical properties of curcumin. Spectrochim Acta A Mol Biomol Spectrosc 67:619-623. doi:10.1016/j.saa.2006.08.018

15. Bong P (2000) Spectral and photophysical behaviors of curcumin and curcuminoids. Bull Chem Soc 21:81-86

16. Khopde SM, Indira Priyadarsini K, Palit DK, Mukherjee $T$ (2007) Effect of solvent on the excited-state photophysical properties of curcumin. Photochem Photobiol 72:625-631. doi:10.1562/0031-8655(2000)0720625

17. Haris P, Mary V, Aparna P et al (2017) A comprehensive approach to ascertain the binding mode of curcumin with DNA. Spectrochim Acta A Mol Biomol Spectrosc 175:155-163. doi:10.1016/j.saa.2016.11.049

18. Pérez E, Ibarra IA, Guzmán A, Lima E (2017) Hybrid pigments resulting from several guest dyes onto $\gamma$-alumina host: a spectroscopic analysis. Spectrochim Acta A Mol Biomol Spectrosc 172:174-181. doi:10.1016/j.saa.2016.04.017

19. Bonab MI, Sardroodi JJ, Ebrahimzadeh AR, Mehrnejad F (2017) A computational study of the electronic structure and the chemical activity of curcumin and some novel curcuminoids by density functional theory. J Iran Chem Soc 14:357-364. doi:10.1007/ s13738-016-0984-x 
20. Anjomshoa S, Namazian M, Noorbala MR (2016) The effect of solvent on tautomerism, acidity and radical stability of curcumin and its derivatives based on thermodynamic quantities. J Solut Chem 45:1021-1030. doi:10.1007/s10953-016-0481-y

21. Margar SN, Rhyman L, Ramasami P, Sekar N (2016) Fluorescent difluoroboron-curcumin analogs: an investigation of the electronic structures and photophysical properties. Spectrochim Acta A Mol Biomol Spectrosc 152:241-251. doi:10.1016/j. saa.2015.07.064

22. Priyadarsini KI, Maity DK, Naik GH et al (2003) Role of phenolic $\mathrm{O}-\mathrm{H}$ and methylene hydrogen on the free radical reactions and antioxidant activity of curcumin. Free Radic Biol Med 35:475-484. doi:10.1016/S0891-5849(03)00325-3

23. Barik A, Priyadarsini KI (2013) Solvent dependent photophysical properties of dimethoxy curcumin. Spectrochim Acta A Mol Biomol Spectrosc 105:267-272. doi:10.1016/j.saa.2012.12.036

24. Nardo L, Andreoni A, Bondani M et al (2012) Studies on curcumin and curcuminoids. XLVI. Photophysical properties of dimethoxycurcumin and bis-dehydroxycurcumin. J Fluoresc 22:597-608. doi:10.1007/s10895-011-0995-z

25. Jasim F, Ali F (1992) A novel and rapid method for the spectrofluorometric determination of curcumin in curcumin spices and flavors. Microchem J 214:209-214

26. Rocha WR, Martins VM, Coutinho K, Canuto S (2002) Solvent effects on the electronic absorption spectrum of formamide studied by a sequential Monte Carlo/quantum mechanical approach. Theor Chem Acc 108:31-37. doi:10.1007/s00214-002-0353-y

27. Coutinho K, Canuto S (2000) Solvent effects in emission spectroscopy: a Monte Carlo quantum mechanics study of the $\mathrm{n} \leftarrow \pi *$ shift of formaldehyde in water. J Chem Phys 113:9132. doi:10.1063/1.1320827

28. Georg HC, Coutinho K, Canuto S (2005) A sequential Monte Carlo quantum mechanics study of the hydrogen-bond interaction and the solvatochromic shift of the $n-\pi^{*}$ transition of acrolein in water. J Chem Phys 123:1-8. doi:10.1063/1.2033750

29. Kirkwood JG (1934) Theory of solutions of molecules containing widely separated charges with special application to zwitterions. J Chem Phys 2:351. doi:10.1063/1.1749489

30. Onsager L (1936) Electric moments of molecules in liquids. J Am Chem Soc 58:1486-1493

31. Tapia O, Goscinski O (1975) Self-consistent reaction field theory of solvent effects. Mol Phys 29:1653-1661. doi:10.1080/00268977500101461

32. Tomasi J, Mennucci B, Cammi R (2005) Quantum mechanical continuum solvation models. Chem Rev 105:2999-3094. doi:10.1021/cr9904009

33. Becke AD (1993) A new mixing of Hartree-Fock and local density-functional theories. J Chem Phys 98:1372. doi: $10.1063 / 1.464304$

34. Lee C, Yang W, Parr RG (1988) Development of the Colle-Salvetti correlation-energy formula into a functional of the electron density. Phys Rev B 37:785-789. doi:10.1103/PhysRevB.37.785
35. Marques MAL, Gross EKU (2004) Time-dependent density functional theory. Annu Rev Phys Chem 55:427-455. doi:10.1146/annurev.physchem.55.091602.094449

36. Adamo C, Barone V (1999) Toward reliable density functional methods without adjustable parameters: the PBE0 model. J Chem Phys 110:6158. doi:10.1063/1.478522

37. Yanai T, Tew DP, Handy NC (2004) A new hybrid exchangecorrelation functional using the Coulomb-attenuating method (CAM-B3LYP). Chem Phys Lett 393:51-57. doi:10.1016/j. cplett.2004.06.011

38. Da Chai J, Head-Gordon M (2008) Long-range corrected hybrid density functionals with damped atom-atom dispersion corrections. Phys Chem Chem Phys 10:6615-6620. doi:10.1039/ b810189b

39. Da Chai J, Head-Gordon M (2008) Systematic optimization of long-range corrected hybrid density functionals. J Chem Phys 128:88106. doi:10.1063/1.2834918

40. Caricato M, Mennucci B, Tomasi J et al (2006) Formation and relaxation of excited states in solution: a new time dependent polarizable continuum model based on time dependent density functional theory. J Chem Phys 124:124520. doi:10.1063/1.2183309

41. Scalmani G, Frisch MJ, Mennucci B et al (2006) Geometries and properties of excited states in the gas phase and in solution: theory and application of a time-dependent density functional theory polarizable continuum model. J Chem Phys 124:94107. doi:10.1063/1.2173258

42. Frisch MJ, Trucks GW, Schlegel HB, et al (2009) Gaussian09

43. Galer P, Golobič A, Koller J et al (2013) Structures in solid state and solution of dimethoxy curcuminoids: regioselective bromination and chlorination. Chem Cent J 7:107. doi:10.1186/1752-153X-7-107

44. Kong X, Brinkmann A, Terskikh V et al (2016) Proton probability distribution in the $\mathrm{O} \cdots \mathrm{H} \cdots \mathrm{O}$ low-barrier hydrogen bond: a combined solid-state NMR and quantum chemical computational study of dibenzoylmethane and curcumin. J Phys Chem B 120:11692-11704. doi:10.1021/acs.jpcb.6b08091

45. Santin LG, Toledo EM, Carvalho-Silva VH et al (2016) Methanol solvation effect on the proton rearrangement of curcumin's enol forms: an $\mathrm{Ab}$ initio molecular dynamics and electronic structure viewpoint. J Phys Chem C 120:19923-19931. doi:10.1021/acs. jpcc.6b02393

46. da Cunha AR, Duarte EL, Lamy MT, Coutinho K (2014) Protonation/deprotonation process of Emodin in aqueous solution and pKa determination: uV/Visible spectrophotometric titration and quantum/molecular mechanics calculations. Chem Phys 440:6979. doi:10.1016/j.chemphys.2014.06.009

47. Shen L, Ji H-F, Zhang H-Y (2005) A TD-DFT study on triplet excited-state properties of curcumin and its implications in elucidating the photosensitizing mechanisms of the pigment. Chem Phys Lett 409:300-303. doi:10.1016/j.cplett.2005.05.023 


\title{
Oxazole Dyes with Potential for Photoluminescence Bioprobes: A Two-Photon Absorption Study
}

\author{
Luis M.G. Abegão, ${ }^{\dagger}$ Ruben D. Fonseca, ${ }^{\ddagger} \S$ Tárcius N. Ramos, ${ }^{\|}$Florence Mahuteau-Betzer, ${ }^{\perp}$ \\ Sandrine Piguel, ${ }^{\perp, \#}$ José Joatan R., Jr., ${ }^{\dagger}$ Cleber R. Mendonça, ${ }^{\ddagger}$ Sylvio Canuto, ${ }^{\|}$Daniel L. Silva, ${ }^{\text {II }}$ \\ and Leonardo De Boni* $*$ ॠ० \\ ${ }^{\dagger}$ Departamento de Física, Universidade Federal de Sergipe, 49100-000 São Cristovão, Sergipe, Brazil \\ ${ }^{\ddagger}$ Instituto de Física de São Carlos, Universidade de São Paulo, CP 369, 13560-970 São Carlos, São Paulo, Brazil \\ ${ }^{\S}$ Universidad de la Costa, Departamento de Ciencias Naturales y Exactas, 58\#55-66, 080002 Barranquilla, Colombia \\ "Instituto de Física, Universidade de São Paulo, Rua do Matão 1371, 05508-090 São Paulo, São Paulo, Brazil \\ ${ }^{\perp}$ Institut Curie, PSL Research University, CNRS, INSERM, UMR9187-U1196, F-91405 Orsay, France \\ \#Université Paris Sud, Université Paris-Saclay, F-91405 Orsay, France \\ "Departamento de Ciências da Natureza, Matemática e Educação, Universidade Federal de São Carlos, Rod. Anhanguera-Km 174, \\ 13600-970 Araras, São Paulo, Brazil
}

Supporting Information

ABSTRACT: In this work, six $\pi$-conjugated oxazole compounds dissolved in dichloromethane were characterized with linear and nonlinear optical measurements. Z-scan with femtosecond laser pulses was employed to determine the two-photon absorption (TPA) spectra. Other photophysical parameters, such as absorbance, solvatochromism, lifetime fluorescence, and fluorescence anisotropy, were evaluated with linear optical techniques. The experimental TPA cross section spectra were adjusted by the sum-over-states (SOS) model, by which important parameters such as transition dipole moments and broadening parameters were determined. To better understand the TPA spectra of the oxazole compounds, quantum-chemical calculations using the response function formalism and the density functional theory level of theory were performed. Using the results provided by the quantum-chemical calculations and the broadening parameters estimated through the application of the SOS model, the TPA spectra were simulated by the superposition (summation) of individual homogeneous Lorentzian absorption profiles.

\section{INTRODUCTION}

Heterocyclic aromatic organic compounds with biological interest have been studied in the nonlinear optics field for their potential application on optical devices, such as multiphoton microscopy, ${ }^{1}$ photodynamic therapy, ${ }^{2}$ and photoluminescence bioprobes (PBs). ${ }^{3}$ The latter technique consists in the detection of a biological analyte by an optical signal, which, in this particular case, is the fluorescence emission signal from a biocompatible organic compound, acting as a signal transducer. ${ }^{4}$ These types of biocompatible optical transducers have been studied for at least 2 decades, in which quantum dots have an important role ${ }^{5}$ and probably still have in the future. Although the toxicity level of the quantum dots is always an unfavorable factor, regardless of their dependence on physicochemical and environmental factors, ${ }^{6}$ it is important to look for new materials with (similar) higher efficiency. The constant presence of this dangerous factor is leading to an urge of searching for new biocompatible compounds, such as organic compounds, with optical and nonlinear optical performances similar to the quantum dots, to be used as fluorescent bioprobes triggered by the two-photon absorption (TPA) process.

Among the variety of natural and synthesized organic compounds, not all of them are suitable for PBs excited by a TPA process. In fact, it happens due to the low probability to transfer population from the ground state to the excited state by $\mathrm{TPA}^{8}$ which must be somehow compensated by a reasonable value of the efficiency quantum yield from the fluorescent emission. Besides the equilibrium between these two optical characteristics, the most suitable organic compounds to be used as PBs by two-photon excitation (PB-TPE) should present low photodamage, low autofluorescence, and high detection sensitivity. Also, the warranty of excitation at a certain depth is

Received: February 24, 2018

Revised: April 10, 2018

Published: April 12, 2018 
<smiles>COc1cc(-c2cnc(-c3cc4ccccc4nn3)o2)cc(OC)c1OC</smiles>

$10 \mathrm{~d}$<smiles>C(=CC1=CC2CCC3CCC2C13)Cc1nnc(-c2ccccc2)o1</smiles>

$12 \mathrm{c}$

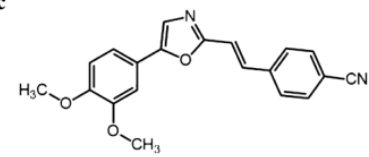

16b

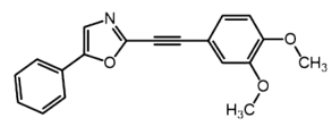

$16 \mathrm{c}$

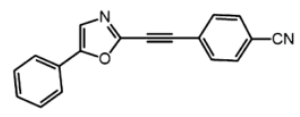

$16 \mathrm{e}$

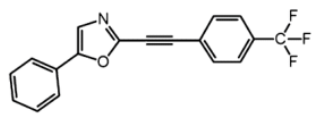

Figure 1. Molecular structures of six 2,5-diaryloxazole derivatives studied.

an extremely important factor, to have the light excitation with low power densities at a certain depth. The presence of these photophysical characteristics are indeed the traditional advantages of PBs via multiphoton excitation compared with single photon excitation. ${ }^{9}$ For example, chalcone derivatives, which have been widely used in biological chemistry activities and possess a satisfactory TPA response, ${ }^{10,11}$ are an example of biocompatible compounds that are unviable to be used as PBTPE. This is due to the extremely low fluorescence quantum yield. On the other hand, recent cell-imaging experiments acquired with oxazole-derivative bioprobes have shown that the fluorescent emission signal can be triggered by one-photon absorption (OPA), leading these type of compounds to be very promising as photoluminescence TPA bioprobes. ${ }^{12}$ Another recent work $^{13}$ has shown a series of oxazole dyes with high efficiency quantum yields, with a medium $\pi$-conjugated molecular size, suggesting that this class of compounds could have a reasonable TPA cross section and consequently the possibility to trigger the fluorescence emission signal by TPA, leading to a promising PB-TPE.

On the basis of these facts, in this work, we present an experimental and theoretical TPA study in the dichloromethane (DCM) medium of six $\pi$-conjugated oxazole dye compounds, which have a 2,5-diaryloxazole unit as their building block, previously synthesized by Mahuteau-Betzer and Piguel, ${ }^{13}$ to understand if this class of molecules are indeed potential compounds to be used as PB-TFE. The Z-scan technique ${ }^{14}$ with tunable femtosecond pulses was used to obtain the TPA spectra. Additional to these measurements, linear optical techniques as solvatochromism, ${ }^{15}$ fluorescence anisotropy, ${ }^{16}$ and fluorescence lifetime were performed to reduce the number of parameters and increase the accuracy on the determination of the transition dipole moment evolved on the singlet ground state to the singlet excited states. The sum-over-state (SOS) approach was used together with the experimental results to fit the TPA spectra and estimate some important spectroscopic parameters of the excited states. Quantum-chemical calculations carried out at the density functional theory (DFT) level were also employed to further understand the TPA spectra behavior. The quantum-chemical calculations were carried out assuming that the compounds were solvated by considering them immersed in a structureless polarizable continuum mimicking the macroscopic properties of the solvent.

\section{EXPERIMENTAL SECTION}

The six molecular structures of the 2,5-diaryloxazole derivatives investigated in this work are displayed in Figure 1. Henceforth, those compounds will be designated as $8 \mathbf{j}\left(\mathrm{C}_{21} \mathrm{H}_{18} \mathrm{~N}_{2} \mathrm{O}_{4}\right), \mathbf{1 0 d}$ $\left(\mathrm{C}_{19} \mathrm{H}_{13} \mathrm{NO}\right)$, 12c $\left(\mathrm{C}_{20} \mathrm{H}_{16} \mathrm{~N}_{2} \mathrm{O}_{3}\right), \mathbf{1 6 b}\left(\mathrm{C}_{19} \mathrm{H}_{15} \mathrm{NO}_{3}\right), \mathbf{1 6}$ $\left(\mathrm{C}_{18} \mathrm{H}_{10} \mathrm{~N}_{2} \mathrm{O}\right)$, and $16 \mathrm{e}\left(\mathrm{C}_{18} \mathrm{H}_{10} \mathrm{~F}_{3} \mathrm{NO}\right)$, the same nomenclature used on ref 13 , in which information regarding the synthesis and preparation can be found.

For linear optical measurements, the samples were placed in $10 \mathrm{~mm}$ thick fused quartz cuvette, whereas in the nonlinear optical measurement a $2 \mathrm{~mm}$ long optical path cuvette was used. The OPA spectra were measured by a UV-vis spectrophotometer system (Shimadzu, model UV-1800), fluorescence emission spectra and the determination of the anisotropy of fluorescence were obtained by a fluorescence spectrophotometer (Hitachi F-4500). Lifetime decay was evaluated by measuring the fluorescence signal as a function of the time with a fast silicon detector ( $0.7 \mathrm{~ns}$ risetime $)$ while the samples were pumped with a $120 \mathrm{fs}$ pulse at $390 \mathrm{~nm}$ (second harmonic generated in a thin barium borate crystal pumped with $150 \mathrm{fs}$ Ti:sapphire laser at 1 $\mathrm{kHz}$ repetition rate). The TPA spectra were measured by the well-known Z-scan technique, ${ }^{14}$ in the open-aperture configuration, in which an optical parametric amplifier (Quantronix, model TOPAS) was employed as a tunable light source. TOPAS was pumped by single pulses of $150 \mathrm{fs}$ from a $1 \mathrm{kHz}$ Ti:sapphire laser (MXR-CLARK). To achieve a Gaussian spatial profile, wavelengths from TOPAS were spatial-filtered with a $50 \mu \mathrm{m}$ diamond pinhole. DCM, tetrahydrofuran, acetonitrile, dimethyl sulfoxide, and toluene, all acquired from Sigma-Aldrich, were used as the solvent media to determine the experimental Stokes shift. The remaining photophysical measurements were acquired in DCM medium. The concentration samples used were of the order of $10^{-5}$ and $10^{-2} \mathrm{M}$ for the linear and nonlinear optical measurements, respectively.

In the Z-scan technique, the relation between the normalized transmittance and the position of the sample with respect to the focal region is given by the following equation: ${ }^{14}$

$$
T(z)=\frac{1}{\sqrt{\pi} q_{0}(z, 0)} \int_{-\infty}^{+\infty} \ln \left[1+q_{0}(z, 0) \mathrm{e}^{-\tau^{2}}\right] \mathrm{d} \tau
$$

in which, $q_{0}(z, 0)=\alpha_{\mathrm{TPA}} I_{0} L\left(1+z^{2} / z_{0}^{2}\right)^{-1}, \alpha_{\mathrm{TPA}}$ is the TPA coefficient, $I_{0}$ is the intensity of the pulse, $L$ is the optical path, $z_{0}$ is the Rayleigh length, and $z$ is the sample position. The experimental Z-scan curves, regarding to the maxima values of 
$\alpha_{\mathrm{TPA}}$, are presented in Figure S1 in the Supporting Information. The experimental TPA cross section $\left(\sigma^{\mathrm{TPA}}\right)$ can be obtained by using the following relation

$$
\sigma^{\mathrm{TPA}}=\frac{h \nu}{N} \alpha^{\mathrm{TPA}}
$$

in which, $h \nu$ is the energy of the incident photon, $N$ is the number of molecules per $\mathrm{cm}^{3}$, and $\alpha^{\text {TPA }}$ is the TPA coefficient. A Göppert-Mayer unit (GM), ${ }^{17}$ which corresponds to $1 \mathrm{GM}=1 \times$ $10^{-50} \mathrm{~cm}^{4} \mathrm{~s}_{\text {photon }}^{-1}$, is commonly used to quantify the $\sigma^{\text {TPA }}$.

In this work, the combination between the optical linear techniques allowed us to obtain the difference between excited and ground states permanent dipole moments $\left(\Delta \mu_{01}=\mu_{11}-\right.$ $\left.\mu_{00}\right)$, by using the Lippert-Mataga equation ${ }^{18}$

$$
\left|\Delta \mu_{01}\right|^{2} \propto \frac{\mathrm{d} \nu_{\mathrm{s}}}{\mathrm{d} F(n, \varepsilon)} a^{3}
$$

which assumes that there exists a direct relationship between $\Delta \mu_{01}$ and the product of the variation solvatochromic shift $\left(\nu_{\mathrm{s}}\right)$ with the Onsager polarity function $(F(n, \varepsilon))$ and the cubic radius of the fluorophore cavity $\left(a^{3}\right)$. Values of $\nu_{\mathrm{s}}$ were extracted from the maxima absorption and emission spectra and are presented in Figure S2 in the Supporting Information. The variation between $\nu_{\mathrm{s}}$ as a function of $F(n, \varepsilon)$ is displayed in Figure S3 also in the Supporting Information, in which $n$ and $\varepsilon$ are the refraction index and dielectric constant from the five solvents used, respectively. The determination of $a^{3}$ was made by using the SmoluchowskiEinstein diffusion equation ${ }^{19}$

$$
V_{\text {hidro }}=\frac{\tau_{\text {emi }} k T}{\eta\left(\frac{0.4}{r}-1\right)}
$$

in which $\tau_{\mathrm{emi}}$ is the fluorescence lifetime (time-resolved fluorescence data are presented in Figure S4), $\eta$ is the solvent viscosity, $r$ is the fluorescence anisotropy, $k$ is the Boltzmann constant, and $T$ is the room temperature. The fluorescence anisotropy was determined by using the following equation: ${ }^{16}$

$$
r=\frac{\frac{I_{\|}}{I_{\perp}}-1}{\frac{I_{\|}}{I_{\perp}}+2}
$$

in which $I$ represents fluorescence emission intensities designated as the intensity parallel and perpendicular to the excitation beam, as $I_{\|}$and $I_{\perp}$, respectively, and the respective plots are presented in Figures 2 and 4 (blue dotted lines).

\section{THEORETICAL SECTION}

The equilibrium geometries of the compounds investigated were obtained performing a geometry optimization calculation and all second derivatives confirmed a minimum energy condition. These calculations were performed using the Gaussian09 software. ${ }^{20}$ To determine the electronic transitions and the excited state properties, quantum-chemical calculations using response formalism, ${ }^{21-23}$ linear function for OPA transitions, and quadratic one for TPA transitions were performed using the Dalton 2016 program. ${ }^{24}$ All the quantum-chemical calculations were performed at the DFT level using the long-range corrected CAM-B3LYP functional ${ }^{25}$ in combination with the 6-311+ $+\mathrm{G}(\mathrm{d}, \mathrm{p})$ basis set.

The solvent interactions were taken into account by employing the polarizable continuum model (PCM) ${ }^{26,27}$ In such an approach the solute is placed in one cavity generated
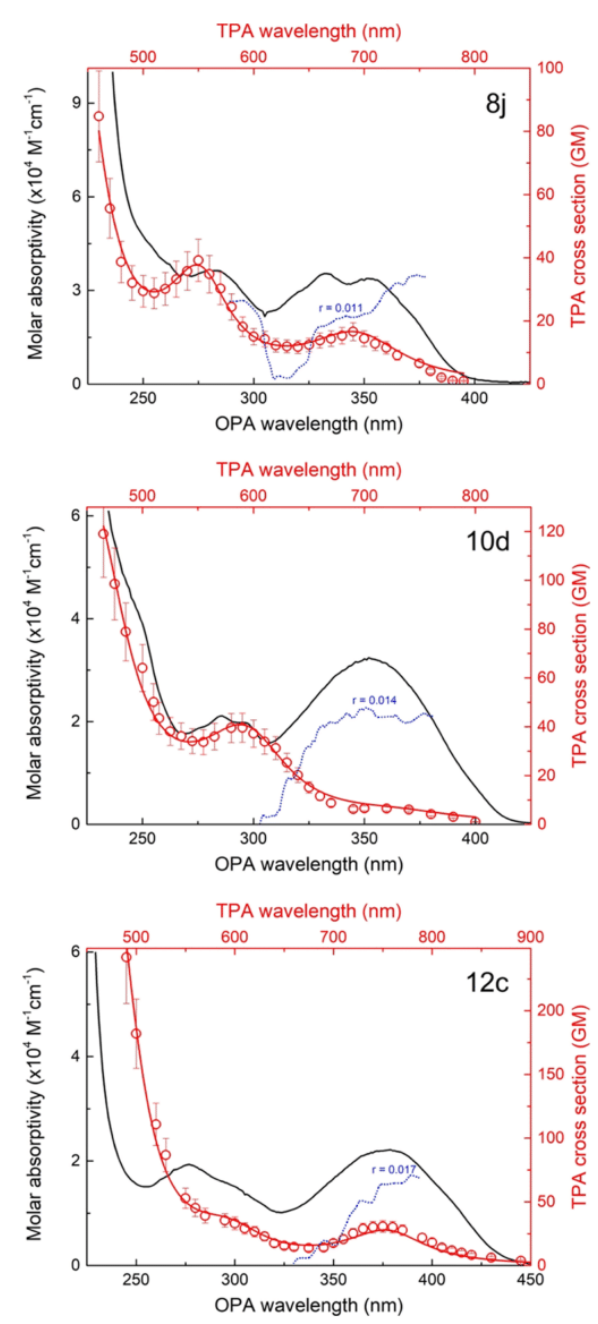

Figure 2. OPA spectra (black line) and experimental TPA (red symbol) spectra of the compounds $8 \mathrm{j}, 10 \mathrm{~d}$, and $12 \mathrm{c}$. The red solid line represents the theoretical fitting obtained with the SOS approach. Blue dotted lines represent excitation anisotropy spectra.

inside a dielectric continuum medium. The cavities were generated using the United Atom Hartree-Fock cavity model, $^{28}$ which uses spheres with radius $10 \%$ higher than the van der Waals radius and centered only on heavy atoms.

The Z-scan technique was used to measure the TPA cross section. For comparing theoretical results with experiment ones, some care was needed. The theoretical TPA spectrum was simulated by taking into account the contribution of the ten lowest-energy excited states. For each transition from the ground (g) to a final (f) excited state, the theoretical $\sigma_{\mathrm{gf}}^{\text {TPA }}$ was obtained considering a single beam setup. The mathematical representations of the TPA are given in atomic units. ${ }^{7,29-31}$

$$
\sigma_{\mathrm{gf}}^{\mathrm{TPA}}=\frac{4 \pi^{3}}{c^{2}} \omega^{2} g\left(2 \omega, \omega_{\mathrm{gf}} ; \Gamma_{\mathrm{f}}\right) \delta_{\mathrm{gf}}
$$

where $\omega_{\mathrm{gf}}$ is the transition energy, $\omega$ is the energy of the incident photon, and $c$ is the light speed. The spectral broadening $g(2 \omega$, $\omega_{\mathrm{g} ;} ; \Gamma_{\mathrm{f}}$ ) is assumed as a Lorentzian shape with linewidth (fullwidth at half-maximum) given by the damping constant $\Gamma_{\mathrm{f}}$. The Lorentzian shape is given by 
Table 1. Spectroscopic Parameters Obtained and Estimated by OPA and TPA Experimental and Theoretical Data for the Studied Samples in DCM Medium (with the Exception of the Parameter Related to the Solvatochromic Shift) ${ }^{a}$

\begin{tabular}{|c|c|c|c|c|c|c|c|}
\hline & spectroscopic parameters & $8 \mathbf{j}$ & $10 \mathrm{~d}$ & $12 \mathrm{c}$ & $16 \mathrm{~b}$ & $16 \mathrm{c}$ & $16 \mathrm{e}$ \\
\hline \multirow[t]{10}{*}{ experimental OPA } & $\varepsilon_{\max }\left(10^{4} \mathrm{M}^{-1} \mathrm{~cm}^{-1}\right)^{b}$ & 3.25 & 3.18 & 2.25 & 4.33 & 2.15 & 2.86 \\
\hline & $\lambda_{\max }^{\mathrm{abs}}(\mathrm{nm})$ & 332 & 355 & 378 & 333 & 335 & 321 \\
\hline & $\lambda_{\max }^{\mathrm{emi}}(\mathrm{nm})$ & 470 & 440 & 496 & 401 & 422 & 408 \\
\hline & $\Phi^{b}$ & 0.76 & 0.32 & 0.38 & 0.04 & 0.28 & 0.24 \\
\hline & $\tau_{\mathrm{emi}}(\mathrm{ns})$ & 2.12 & 0.86 & 1.10 & 0.77 & 1.04 & 0.71 \\
\hline & $a^{3}\left(\mathrm{~A}^{3}\right)$ & 146 & 72 & 118 & 260 & 105 & 90 \\
\hline & $r$ & 0.011 & 0.014 & 0.017 & 0.049 & 0.025 & 0.016 \\
\hline & $\mathrm{d} \nu_{s} / \mathrm{d} F(n, \varepsilon)$ & 4245 & 1223 & 3785 & 1382 & 3771 & 3054 \\
\hline & $\Delta \mu_{01}(\mathrm{D})$ & 8.21 & 2.92 & 6.26 & 5.73 & 6.81 & 5.12 \\
\hline & $\mu_{01}(\mathrm{D})$ & 4.06 & 5.74 & 5.85 & 6.02 & 4.95 & 5.02 \\
\hline \multirow[t]{2}{*}{ SOS } & $\mu_{12}(\mathrm{D})$ & 6.69 & 7.72 & 4.21 & 6.71 & 10.04 & 8.38 \\
\hline & $\mu_{13}(\mathrm{D})$ & 7.45 & 9.11 & 8.19 & 8.22 & 9.07 & \\
\hline \multirow[t]{4}{*}{ experimental TPA } & $\lambda_{0-1}^{\mathrm{TPA}}(\mathrm{nm})$ & 690 & 700 & 750 & 630 & 645 & 630 \\
\hline & $\sigma_{0-1}^{\mathrm{TPA}}\left[\sigma_{0-1}^{\prime \mathrm{TPA}}\right](\mathrm{GM})$ & $17[13]$ & $22[7]$ & $30[11]$ & $15[1]$ & $28[8]$ & $16[4]$ \\
\hline & $\lambda_{0-2}^{\mathrm{TPA}}(\mathrm{nm})$ & 550 & 580 & 570 & 530 & 570 & 525 \\
\hline & $\sigma_{0-2}^{\mathrm{TPA}}\left[\sigma_{0-2}^{\prime \mathrm{TPA}}\right](\mathrm{GM})$ & $39[30]$ & $39[12]$ & $39[15]$ & $58[2]$ & $85[24]$ & $58[14]$ \\
\hline \multirow[t]{4}{*}{ theoretical TPA } & $\lambda_{0-1}^{\mathrm{TPA}}(\mathrm{nm})$ & 660 & 712 & 754 & 672 & 684 & 664 \\
\hline & $\sigma_{0-1}^{\mathrm{TPA}}(\mathrm{GM})$ & 15 & 0 & 75 & 2 & 42 & 23 \\
\hline & $\lambda_{0-2}^{\mathrm{TPA}}(\mathrm{nm})$ & 572 & 578 & 566 & 540 & 526 & 514 \\
\hline & $\sigma_{0-2}^{\mathrm{TPA}}(\mathrm{GM})$ & 8 & 10 & 0 & 33 & 28 & 29 \\
\hline
\end{tabular}

${ }^{a} \Phi$ is the fluorescence quantum yield. The theoretical values for the TPA cross section of the transitions were computed, assuming the damping constant values shown in the Table S1. ${ }^{b}$ From ref 13.

$$
g\left(2 \omega, \omega_{\mathrm{gf}} ; \Gamma_{\mathrm{f}}\right)=\frac{1}{2 \pi} \frac{\Gamma_{\mathrm{f}}}{\left(2 \omega-\omega_{\mathrm{gf}}\right)^{2}+0.25 \Gamma_{\mathrm{f}}^{2}}
$$

while the TPA transition probability $\delta_{\text {gf }}$ for a linear polarized source in an isotropic media is defined as

$$
\delta_{\mathrm{gf}}=\frac{1}{15} \sum_{\alpha \alpha}\left\{S_{\alpha \beta}^{\mathrm{gf}}\left(S_{\beta \beta}^{\mathrm{gf}}\right)^{*}+2 S_{\alpha \beta}^{\mathrm{gf}}\left(S_{\alpha \beta}^{\mathrm{gf}}\right)^{*}\right\}
$$

where $S_{\alpha \beta}^{\mathrm{gf}}$ represents the two-photon matrix elements for the TPA degenerated process and are given as

$$
S_{\alpha \beta}^{\mathrm{gf}}=\sum_{k}\left\{\frac{\left\langle g\left|\mu_{\alpha} \mu_{\beta}\right| f\right\rangle}{\omega_{\mathrm{gk}}-\omega}+\frac{\left\langle g\left|\mu_{\beta} \mu_{\alpha}\right| f\right\rangle}{\omega_{\mathrm{gk}}-\omega}\right\}
$$

where the $\alpha$ and $\beta$ represent the Cartesian coordinates. The simulated TPA spectra were obtained by summing the contribution of the main low-lying TPA transitions employing the $\Gamma_{\mathrm{f}}$ values estimated through the SOS fitting. The theoretical values are presented in Table 1 . The conversion factor from atomic units (au) to GM is 1.896788 .

To improve the TPA spectral analysis, the theoretical SOS method was used to fit the experimental TPA spectra with the following equation: ${ }^{32,33}$

$$
\begin{aligned}
\sigma_{\mathrm{SOS}}^{\mathrm{TPA}}(\nu)= & \frac{4}{5 \pi} \frac{(2 \pi)^{4}}{(c h)^{2}} L^{4} \frac{\nu^{2}}{\left(\nu_{01}-\nu\right)^{2}+\Gamma_{01}{ }^{2}} \\
& {\left[\frac{\left|\mu_{01}\right|^{2} \Delta \mu_{01}{ }^{2} \Gamma_{01}}{\left(\nu_{01}-2 \nu\right)^{2}+\Gamma_{01}{ }^{2}}+\frac{\left|\mu_{12}\right|^{2}\left|\mu_{01}\right|^{2} \Gamma_{02}}{\left(\nu_{02}-2 \nu\right)^{2}+\Gamma_{02}{ }^{2}}\right.} \\
& \left.+\frac{\left|\mu_{13}\right|^{2}\left|\mu_{01}\right|^{2} \Gamma_{03}}{\left(\nu_{03}-2 \nu\right)^{2}+\Gamma_{03}{ }^{2}}\right]
\end{aligned}
$$

in which $\nu$ is the frequency of the laser, $c$ is the speed of light, $L$ is the local correction factor, and $h$ is the Planck constant. The spectroscopic parameters, $\nu_{0 n}, \Gamma_{0 n}$ and $\mu_{0 n}$ correspond to the transition frequency, damping constant, and transition dipole moments, respectively, of the $0 \rightarrow n$ transition $(n=1,2)$, while the difference between permanent dipole moments of the excited and ground states of the molecule is given by $\Delta \mu_{01}$. The transition dipole moment between the ground and the first excited state $\left(\mu_{01}\right)$ can be evaluated from OPA spectra using the following relationship: ${ }^{33}$

$$
\mu_{01}=\sqrt{\frac{3}{8 \pi^{3}} \frac{c h}{N} \frac{1}{\nu_{01}} \int \alpha(\nu) \mathrm{d} \nu}
$$

in which, $N$ is the number of molecules per $\mathrm{cm}^{3}, \alpha$ is the absorption coefficient in $\mathrm{cm}^{-1}$. It is important to say that in eq 10 the only parameters that were obtained by adjusting the TPA spectra were $\mu_{12}, \Gamma_{02}, \mu_{13}$ and $\Gamma_{03}$, and these parameters are independently adjusted. Later, this point will be clarified together with the TPA spectra. Table 1 summarizes the spectroscopic parameters provided by the SOS fitting, considering that $\mu_{12}$ and $\mu_{13}$ are the estimated adjusted parameters retrieved by the SOS approach.

\section{RESULTS AND DISCUSSION}

The experimental OPA spectrum of the compound $8 \mathbf{j}$ shows a higher energy band centered at ca. $280 \mathrm{~nm}$ and a lower energy band, almost with the same intensity, centered at approximately $350 \mathrm{~nm}$, as shown in Figure 2. The latter band is related to the quinoline group, where the presence of the nitrogen affects the charge distribution in both heterocyclic rings, creating an asymmetry of charge, reducing the density of $\pi$-electrons in the benzene ring. ${ }^{34}$ This charge asymmetry allows that the dipole selection rules to be relaxed. Consequently, it is expected that the TPA spectrum has a similar spectral characteristic of the OPA, as one can see a clearly spectral correlation between the OPA and the TPA spectra with two well-defined bands. In other words, the TPA spectral line shape follows almost the same behavior of its analog of one-photon, showing that states accessed via one- 
photon are also accessed via two-photons due to relaxation of the selection rules, ${ }^{32,35}$ which is in accordance with the asymmetrical nature of the molecular structure of the $\mathbf{8 j}$ compound. The previous mentioned absorption bands, for this compound, have the same molar absorptivity; however, the less energetic band has a maximum $\sigma^{\mathrm{TPA}}$ two times smaller than the one located at ca. $270 \mathrm{~nm}$, revealing that even with the charge asymmetry provided by the quinoline heterocycle structure, this transition is less allowed by two-photons than one-photon.

Experimental TPA and OPA spectra of compound 10d also present two electronic transitions allowed by one-photon centered at ca. $286 \mathrm{~nm}$ corresponding to the higher energetic transition, while the lower energetic transition is centered at ca. $355 \mathrm{~nm}$, as one can see in Figure 2. By comparison with compound $8 \mathbf{j}$, it is possible to see that the higher energetic band presents a reduction of the molar absorptivity of about 1.5 times. Additionally, the TPA spectrum does not match the OPA spectrum, where the lower energetic OPA band does not have its correspondence in the TPA spectrum. This correspondence absence could be associated to the less charge asymmetry of the naphthalene group compared to the quinolone moiety. Probably a less asymmetrical nature of the compound $10 \mathrm{~d}$ leads to a weaker relaxation of the selection rules, which makes this particular one photon transition less probable by two-photons. The experimental $\sigma^{\mathrm{TPA}}$ highest value achieved is located at ca. 580 $\mathrm{nm}$ (excitation wavelength), which corresponds to the energy of the higher energy band allowed by one-photon. The magnitude of this band matches the same experimental value of the equivalent band from compound $\mathbf{8 j}$.

Compound 12c seems to have a less asymmetric molecular structure compared with $8 \mathbf{j}$ and $\mathbf{1 0 d}$, in which the quinoline and naphthalene groups are replaced by 4-cyanophenyl. As discussed previously, less symmetric charge distribution gives an higher probability from having a relaxation of the selection rules, ${ }^{36}$ consequently, one could expect that electronic transitions of this compound allowed by one-photon are also allowed by twophotons, which is indeed observed in Figure 2. The most intense transitions allowed by one-photon, which have its maximum of probability at ca. $275 \mathrm{~nm}$ and at ca. $375 \mathrm{~nm}$, are also allowed by two-photons with maxima experimental $\sigma^{\text {TPA }}$ values at 570 and $750 \mathrm{~nm}$. This spectral behavior is in accordance with previous studies $^{30,35,37}$ and excepted for compounds that have an asymmetric molecular structure nature.

The transitions observed on the TPA spectra are corroborated by the excitation anisotropy displayed in the same Figure 2 (dotted blue lines). Particularly for molecule $10 \mathrm{~d}$, it is possible to see a constant value on the excitation anisotropy $(r)$ between 325 and $390 \mathrm{~nm}$, which is attributed to a transition from the groundstate $\left(\mathrm{S}_{0}\right)$ to the first singlet excited state $\left(\mathrm{S}_{1}\right)$. A change in the excitation anisotropy value for shorter wavelengths than $325 \mathrm{~nm}$ indicates that a second excited state $\left(S_{2}\right)$ is reached. The previously described behaviors were also observed for the compounds $8 \mathrm{j}$ and $12 \mathrm{c}$.

Concerning the theoretical OPA spectrum of the compound $8 \mathbf{j}$, the calculated results pointed out that, despite its structure, a single OPA-allowed transition (HOMO $\rightarrow$ LUMO, 29.6\%) is responsible for the lowest-energy band in the spectrum. The higher energy band (centered at $285 \mathrm{~nm}$ ), on the other hand, would be the result of the summation effect of three transitions. The theoretical results indicate that the bands observed in the TPA spectrum (centered at 345 and $275 \mathrm{~nm}$ ) of the compound $8 \mathbf{j}$ are related, each of them, to a single transition, that is, the access to a single excited state. On the other hand, the enhancement of the TPA cross section from the region $\lambda>$ $250 \mathrm{~nm}$ should be ascribed to the presence of two excited states of higher energy and strongly TPA allowed.

As it will be evidenced later, for all compounds investigated the lowest energy band of the OPA spectrum is related to the lowestenergy transition of the compound. Because the most important contribution for that transition is the HOMO $\rightarrow$ LUMO excitation, such molecular orbitals are shown in Figure 3.

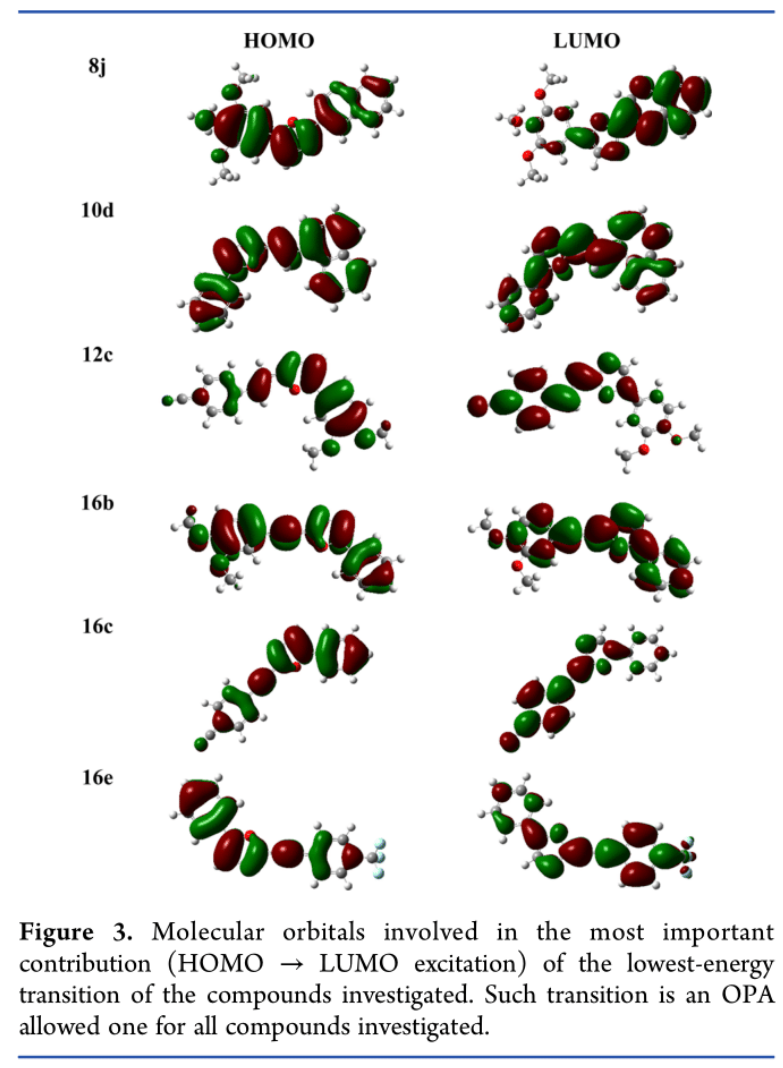

For the compound 10d, theoretical results pointed out that the lowest-energy band in the simulated OPA spectrum is the result of a single transition (HOMO $\rightarrow$ LUMO, 42.2\%), the lowestenergy transition of the compound. The higher energy band (centered at $287 \mathrm{~nm}$ ) should also be ascribed to a single OPAallowed transition but much less intense than the lowest-energy transition of the compound. The theoretical results confirmed that there is no TPA-allowed transition in the spectral region around $350 \mathrm{~nm}$ and also that the band centered at $285 \mathrm{~nm}$ in the TPA spectrum of the compound $10 \mathrm{~d}$ is related to a single transition. Moreover, the theoretical results indicate that the increase of the TPA cross section for the region of $\lambda<260 \mathrm{~nm}$ should be ascribed to the three higher energy excited states strongly TPA allowed.

Concerning the simulated OPA spectrum of the compound $12 \mathrm{c}$, the theoretical results pointed out that a single transition (HOMO $\rightarrow$ LUMO, 32.5\%), the lowest-energy one, is responsible for the lowest-energy band in the OPA spectrum. The higher energy band (centered at $285 \mathrm{~nm}$ ) clearly presents an asymmetric profile. The theoretical results pointed out that there are four OPA-allowed transitions along the spectral region of such band. For the compound 12c, the theoretical results indicate that the TPA band centered at $378 \mathrm{~nm}$ is related to a 
single excited state of the compound, the lowest-energy excited state and that is OPA-allowed also. A band in the higher energy region of the TPA spectrum is also observed. The shape of such band, centered at ca. $290 \mathrm{~nm}$, is severely affected by the abrupt increase of the TPA cross section because of either the presence of other TPA-allowed excited states energetically close or the resonance enhancement (due to the OPA process). The theoretical results pointed out a TPA-allowed transition at the $283 \mathrm{~nm}$ region to which one can associate the TPA band centered at ca. $290 \mathrm{~nm}$ and a TPA-allowed transition at ca. $260 \mathrm{~nm}$ region that can explain the increase of the TPA cross section for $\lambda<275$ $\mathrm{nm}$.

Comparable experimental study was made for compounds 16b, 16e, and 16c, which are similar from each other and distinct from $8 \mathrm{j}, 10 \mathrm{~d}$, and $12 \mathrm{c}$. It can be seen on the molecular structures that the differences between these three compounds are the substituents on the phenyl rings. However, the compounds are more conjugated if compared to the first three molecular structures $(\mathbf{8 j}, 10 \mathrm{~d}$, and $12 \mathrm{c})$ because of the triple bonds. Previous studies ${ }^{38,39}$ already have shown that a more extended $\pi$ conjugation favors the response of TPA, and consequently, it is expected that compounds $16 \mathrm{~b}, 16 \mathrm{e}$, and $16 \mathrm{c}$ present higher values of experimental $\sigma^{\mathrm{TPA}}$ than compounds $8 \mathrm{j}, 10 \mathrm{~d}$, and $12 \mathrm{c}$. Additional, to the extension of the $\pi$-conjugation interfering on the $\sigma^{\text {TPA }}$ values, for example, the position of the substituents on the phenyl ring could increase those values as well, ${ }^{40}$ in particular, if the substituents are in the para position of the ring. Considering these facts, it is expected that the experimental $\sigma^{\text {TPA }}$ values of compounds $16 \mathrm{~b}, 16 \mathrm{e}$, and $16 \mathrm{c}$ are higher than the ones obtained for $8 \mathrm{j}, 10 \mathrm{~d}$, and 12c. Indeed, the highest $\sigma^{\mathrm{TPA}}$ value achieved was obtained on the compound 16c, which presents the cyano group at the para position of the phenyl ring, demonstrating an accordance with the previous mentioned literature. ${ }^{40}$

The line shape of the experimental OPA spectra is similar to those of these three compounds, in which the electronic transition with higher intensity corresponds ca. to 333,335 , and $321 \mathrm{~nm}$ for the compounds $16 \mathrm{~b}, 16 \mathrm{e}$, and $16 \mathrm{c}$, respectively. Additionally, one can see that this band presents a shoulder centered ca. at 359,362 , and $347 \mathrm{~nm}$ for the compounds $\mathbf{1 6 b}$, $16 \mathrm{e}$, and 16c, respectively. Moreover, a higher transition is located at a wavelength about $280 \mathrm{~nm}$ for all three compounds, with smaller absorption intensity when compared to the lowest energetic band. However, both bands have opposite behavior on the TPA intensities. The smaller OPA band is responsible for the higher TPA cross section values obtained for this class of molecules. The compounds $16 \mathrm{~b}$ and $16 \mathrm{e}$ also have the experimental $\sigma^{\mathrm{TPA}}$ maxima values located at the same electronic state. Also, one can see that the excitation anisotropy displayed at the same Figure 4 (dotted blue lines) shows different regions, one located between $300 \mathrm{~nm}$ up to 360 , indicating a transition $\mathrm{S}_{0-1}$. A strong decrease in the excitation anisotropy for wavelengths shorter than $300 \mathrm{~nm}$, which matches with the second TPA band, shows that a new transition is responsible for the TPA on this spectral range. Consequently, the second TPA band is assigned by a higher energy transition.

For the compound $\mathbf{1 6 b}$, the theoretical results pointed out that the lowest-energy band in the OPA spectrum is the result of a single transition (HOMO $\rightarrow$ LUMO, 39.8\%), the lowest-energy transition of the compound. The higher energy band (centered at $265 \mathrm{~nm}$ ) in the OPA spectrum is much less intense than the lowest-energy one and should be ascribed to two OPA-allowed transitions with modest oscillator strengths ( 0.10 and 0.07$)$. The theoretical results indicate that the subtle and striking bands
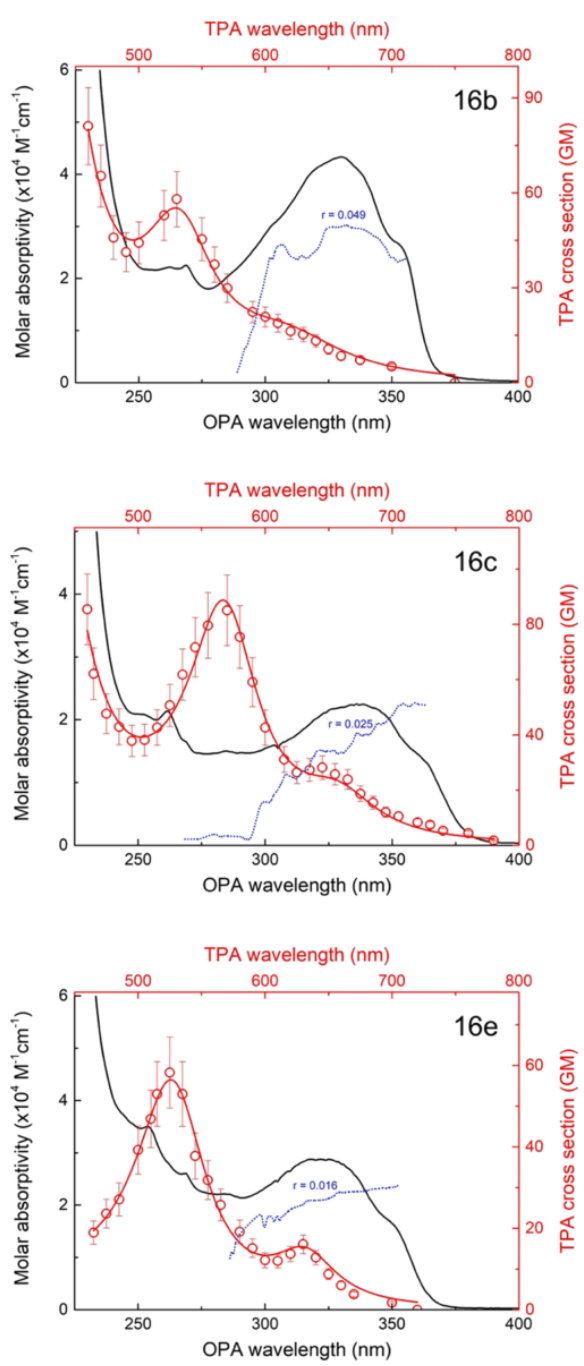

Figure 4. OPA spectra (black line) and experimental TPA (red symbol) spectra of the compounds 16b, 16c, and 16e. The red solid line represents the theoretical fitting obtained with the SOS approach. Blue dotted lines represent excitation anisotropy spectra.

observed in the TPA spectrum of the compound $16 \mathbf{b}$, respectively, at 320 and $265 \mathrm{~nm}$, should be related to a single excited state each of them. The increase of the TPA cross section from $\lambda<250 \mathrm{~nm}$ region should be ascribed to the presence of two higher energy excited states strongly TPA allowed.

Regarding compound 16c, the theoretical results pointed out that a single transition (HOMO $\rightarrow$ LUMO, 36.0\%), the lowestenergy one, is responsible for the lowest-energy band in the OPA spectrum. In the $250-300 \mathrm{~nm}$ region of the spectrum, no welldefined band is observed, but the quantum-chemical calculation indicates that two OPA-allowed transitions act along the higher energy part of that spectral region. The theoretical results pointed out that the bands observed in the TPA spectrum (centered at 325 and $285 \mathrm{~nm}$ ) of the compound 16c are related, each of them, to a single TPA transition. The difference between the theoretical energy transitions and the energy related with the central wavelength of those bands indicates that the PCM/CAMB3LYP approach probably did not stabilize satisfactorily the energy of the electronic excited states involved in such 

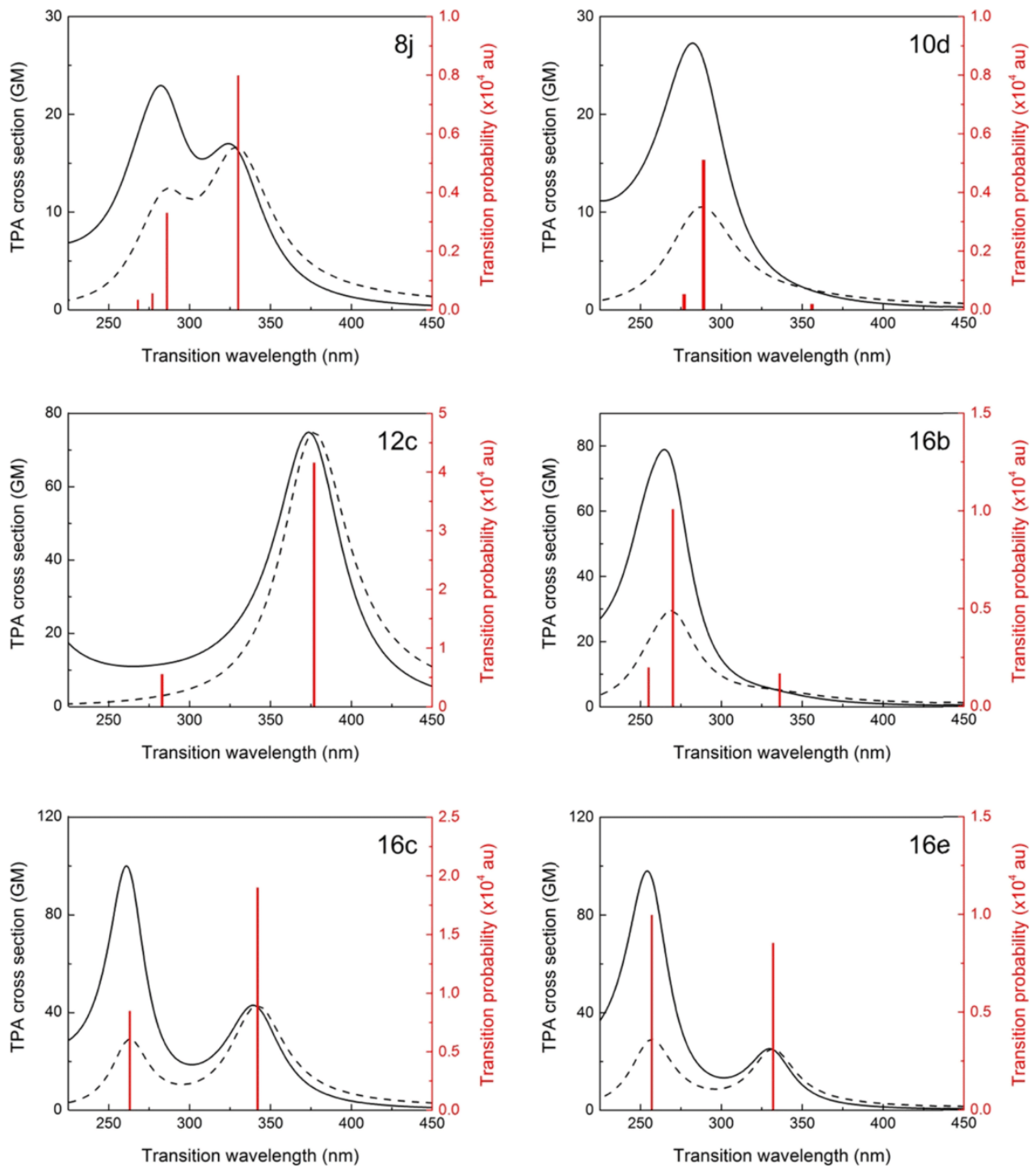

Figure 5. Simulated TPA spectra. Solid line describes the TPA spectra taking into account resonance enhancement due to the OPA process and dashed line without the resonance effect. The vertical lines illustrate the TPA probabilities of the transitions considered to simulate the spectra. The linewidths on the simulations were used as the same obtained experimentally in the homogeneous (Lorentzian) absorption profile.

transitions. The increase of the TPA cross section from $\lambda<250$ $\mathrm{nm}$ region should be mainly attributed to the presence of one excited state of higher energy that is strongly TPA allowed.

For the compound $16 \mathrm{e}$, the theoretical results pointed out that a single transition (HOMO $\rightarrow$ LUMO, 38.9\%) is responsible for the lowest-energy band in the OPA spectrum, the lowest-energy one. Again, despite the high and increasing molar absorptivity in the $250-290 \mathrm{~nm}$ region of the spectrum, no well-defined band is observed there. The quantum-chemical calculation indicates that a single OPA-allowed transition is the primarily responsible for such part of the OPA spectrum. The theoretical results for the compound 16e indicated that a single TPA-allowed transition is responsible for each of the two bands observed in the TPA spectrum (centered at 320 and $265 \mathrm{~nm}$ ). In addition, the theoretical results indicated that transitions via TPA should still be observed in the $225-235 \mathrm{~nm}$; however, no evidences are observed in the experimental spectrum.
As mentioned in the theoretical section, the experimental TPA spectra were fitted by the SOS phenomenological approach by using the eq 10, which provided the estimation of the spectroscopic parameters $\mu_{01}, \mu_{12}$, and $\mu_{13}$ presented in Table 1. On the basis of the results of the quantum-chemical calculations, it is possible to realize that actually for some of the compounds investigated, the third and/or fourth states of our SOS model (second and third excited states of the compound) must be related to the role of more than one electronic excited state of the system. Therefore, it is more correct to state that the excited states of our SOS model are describing absorption bands rather than real electronic states. On the other hand, the results of the quantum-chemical calculations support that it is coherent to assume that the second state in our SOS model describes the first excited state of each compound investigated.

The value of $\Delta \mu_{01}$ could also be estimated by the SOS approach; however, here we used the one determined 
experimentally by using eq 3 , which have implied measuring the fluorescence anisotropy, the emission lifetime decay, and the variation of the Stokes shift as a function of the Onsager polarity function. To fully understand how these compounds could be used as PB-TPE, it is important to calculate the value of their brightness, defined by Drobizhev et al., ${ }^{41}$ as the TPE action cross section $\left(\sigma^{\prime \text { TPA }}\right)$, which is directly related to the product between $\sigma_{\mathrm{TPA}}^{\exp }$ and $\Phi$. All these experimental values are also presented in Table 1 .

The two-photon spectral region with more probability to trigger fluorescence emission, with half of the energy needed by one-photon excitation, is between 600 and $700 \mathrm{~nm}$. At this region, the maximum $\sigma_{\mathrm{TPA}}^{\exp }$ measured belongs to the compounds $12 \mathrm{c}$ and 16c, corresponding approximately to 30 and $28 \mathrm{GM}$, respectively, while the compound $8 \mathbf{j}$ presents around 17 GM. The fluorescence quantum yields of $8 \mathbf{j}, \mathbf{1 2 c}$, and $16 \mathrm{c}$ are 0.76 , 0.38 , and $0.28{ }^{13}$ respectively, in which $8 \mathbf{j}$ has the maximum value of brightness ${ }^{41}$ corresponding to $13 \mathrm{GM}$, followed by $12 \mathrm{c}$ with a brightness value of $11 \mathrm{GM}$, whereas the $16 \mathrm{c}$ has only a brightness value of $8 \mathrm{GM}$. However, in the TPA spectral region between 530 and $580 \mathrm{~nm}$, the maximum $\sigma_{\mathrm{TPA}}^{\exp }$ values are found for the compounds, in which $8 \mathbf{j}$ and $16 \mathrm{c}$ present the values of 39 and 85 GM, respectively. If, in this spectral region, the fluorescence is also triggered with the same quantum yield, then the brightness values of the latter compounds are 30 and $24 \mathrm{GM}$, respectively. It shows that equilibrium between the optical variables TPA and quantum yield is the best way to achieve the optimal compounds to be used as PB-TPE. None of the studied compounds have presented undesirable degradation or bleaching, which are also important optical characteristics for a PB-TPE.

Using the results provided by the quantum-chemical calculations and the broadening parameters estimated through the application of the SOS model, theoretical values for the TPA cross section of the main excited state related to each band in the TPA spectrum of the compounds were calculated. The values are shown in the Table 1. Comparing with the experimental values, one can note that for the spectral region of the low energy band $\left(\sigma_{0-1}^{\mathrm{TPA}}\right)$ the theoretical results predict good values for the TPA cross section of the compounds $8 \mathrm{j}, 16 \mathrm{c}$, and $16 \mathrm{e}$, underestimate the TPA cross section of the compounds $10 \mathrm{~d}$ and $16 \mathrm{~b}$, and overestimate the TPA cross section of the compound $12 \mathrm{c}$. In addition, one can note that for the spectral region of the higher energy band $\left(\sigma_{0-2}^{\mathrm{TPA}}\right)$ the theoretical results underestimate the TPA cross sections of most TPA-allowed transitions; the exception is the case of compound $\mathbf{1 6 b}$.

All the TPA transitions (listed in the Table 1) determined by the quantum-chemical calculations and energetically located in the spectral region of the two sharp bands of the TPA spectrum were considered to simulate the nonlinear absorption spectrum of the compounds investigated. The TPA spectra were simulated by the superposition (summation) of individual homogeneous (Lorentzian) absorption profiles, with linewidths given by the values of the broadening parameters. The spectra were simulated, assuming two possible situations: (1) the resonance enhancement effect (due to the OPA process) is negligible and (2) the resonance enhancement effect is important and it is taken into account by multiplying the theoretical values of the TPA cross sections by the frequency-dependent factor outside the brackets in our SOS expression (eq 10). The simulated spectra are presented in Figure 5.

From a direct comparison with the experimental TPA spectra, one can conclude that it is important to consider the resonance enhancement effect to obtain a reasonable agreement between experimental and simulated spectra, concerning the ratio between the amplitudes of the bands observed in the TPA spectra for some of the compounds investigated.

\section{CONCLUSIONS}

The combination between linear and nonlinear photophysical parameters investigated in this work revealed that oxazoles dyes, in particular the ones presented in this study, could be a new generation of PBs via TPA. In fact, none of the samples presented photobleaching which is one of the most desirable characteristics in PBs. The simulated TPA spectra are in accordance with the experimental ones in the spectral region and with higher probability of absorbing two-photons. Moreover, the experimental determination of the TPA cross section values, combined with the fluorescence quantum yield, allows us to identify the best spectral region where the studied compounds could be most suitably used as PB-TPE. Their maximum fluorescence emission, triggered by the excitation in the near-infrared region, ranging from 408 to $496 \mathrm{~nm}$, is suitable for fluorescence bioimaging acquisition by using the blue channel of a charged couple device. In fact, this work should trigger a future joint research with other fields of science, such as biology, to study the biocompatible performance of the oxazole dyes, as well as to record in vivo their TPE, to compare with the obtained results.

\section{ASSOCIATED CONTENT}

S Supporting Information

The Supporting Information is available free of charge on the ACS Publications website at DOI: 10.1021/acs.jpcc.8b01904.

Open aperture Z-scan signatures associated with the maximum values of TPA cross sections in DCM solution; normalized absorption and fluorescence spectra in five different solvents; solvatochromic Stoke shifts $(\nu)$ measurements obtained as a function of the Onsager polarity function $(F(n, \varepsilon))$; normalized absorption spectra and fluorescence anisotropy measurements in DCM; fluorescence life time decay in DCM solution; and theoretical results for the OPA and TPA transitions for the six compounds studied in DCM in this work (PDF)

\section{AUTHOR INFORMATION}

\section{Corresponding Author}

*E-mail: deboni@ifsc.usp.br. Phone: +55-16-33738085.

ORCID

Leonardo De Boni: 0000-0002-1875-1852

Notes

The authors declare no competing financial interest.

\section{ACKNOWLEDGMENTS}

The authors acknowledge the financial support from FAPESP (Fundação de Amparo à Pesquisa do Estado de São Paulo 2011/ 12399-0, 2015/20032-0, 2015/14189-3 and 2016/20886-1) and CNPq (Conselho Nacional de Desenvolvimento Científico e Tecnológico).

\section{REFERENCES}

(1) Zipfel, W. R.; Williams, R. M.; Webb, W. W. Nonlinear Magic: Multiphoton Microscopy in the Biosciences. Nat. Biotechnol. 2003, 21, 1369.

(2) Kamkaew, A.; Lim, S. H.; Lee, H. B.; Kiew, L. V.; Chung, L. Y.; Burgess, K. Bodipy Dyes in Photodynamic Therapy. Chem. Soc. Rev. 2013, 42, 77-88. 
(3) Ding, D.; Li, K.; Liu, B.; Tang, B. Z. Bioprobes Based on Aie Fluorogens. Acc. Chem. Res. 2013, 46, 2441-2453.

(4) Turner, A.; Karube, I.; Wilson, G. S. Biosensors: Fundamentals and Applications; Oxford University Press, 1987.

(5) Algar, W. R.; Tavares, A. J.; Krull, U. J. Beyond Labels: A Review of the Application of Quantum Dots as Integrated Components of Assays, Bioprobes, and Biosensors Utilizing Optical Transduction. Anal. Chim. Acta 2010, 673, 1-25.

(6) Hardman, R. A Toxicologic Review of Quantum Dots: Toxicity Depends on Physicochemical and Environmental Factors. Environ. Health Perspect. 2006, 114, 165.

(7) Boyd, R. W. Nonlinear Optics; Academic Press, 2003.

(8) Brakenhoff, G. J.; Müller, M.; Ghauharali, R. I. Analysis of Efficiency of Two-Photon Versus Single-Photon Absorption for Fluorescence Generation in Biological Objects. J. Microsc. 1996, 183, 140-144.

(9) Bush, P. G.; Wokosin, D. L.; Hall, A. C. Two-Versus One Photon Excitation Laser Scanning Microscopy: Critical Importance of Excitation Wavelength. Front. Biosci. 2007, 12, 2646.

(10) Abegão, L. M. G.; Fonseca, R. D.; Santos, F. A.; Souza, G. B.; Barreiros, A. L. B. S.; Barreiros, M. L.; Alencar, M. A. R. C.; Mendonça, C. R.; Silva, D. L.; De Boni, L. Second-and Third-Order Nonlinear Optical Properties of Unsubstituted and Mono-Substituted Chalcones. Chem. Phys. Lett. 2016, 648, 91-96.

(11) Gu, B.; Ji, W.; Patil, P. S.; Dharmaprakash, S. M.; Wang, H.-T. Two-Photon-Induced Excited-State Absorption: Theory and Experiment. Appl. Phys. Lett. 2008, 92, 091118.

(12) Dias, G. G.; Pinho, P. V. B.; Duarte, H. A.; Resende, J. M.; Rosa, A. B. B.; Correa, J. R.; Neto, B. A. D.; da Silva Júnior, E. N. Fluorescent Oxazoles from Quinones for Bioimaging Applications. RSC Adv. 2016, 6, 76056-76063.

(13) Mahuteau-Betzer, F.; Piguel, S. Synthesis and Evaluation of Photophysical Properties of Series of П-Conjugated Oxazole Dyes. Tetrahedron Lett. 2013, 54, 3188-3193.

(14) Sheik-Bahae, M.; Said, A. A.; Wei, T.-H.; Hagan, D. J.; Van Stryland, E. W. Sensitive Measurement of Optical Nonlinearities Using a Single Beam. IEEE J. Quantum Electron. 1990, 26, 760-769.

(15) Nigam, S.; Rutan, S. Principles and Applications of Solvatochromism. Appl. Spectrosc. 2001, 55, 362A-370A.

(16) Lakowicz, J. R.; Masters, B. R. Principles of Fluorescence Spectroscopy. J. Biomed. Opt. 2008, 13, 029901.

(17) Göppert-Mayer, M. Elementary Processes with Two Quantum Transitions. Ann. Phys. 2009, 18, 466-479.

(18) Makarov, N. S.; Mukhopadhyay, S.; Yesudas, K.; Brédas, J.-L.; Perry, J. W.; Pron, A.; Kivala, M.; Müllen, K. Impact of Electronic Coupling, Symmetry, and Planarization on One-and Two-Photon Properties of Triarylamines with One, Two, or Three Diarylboryl Acceptors. J. Phys. Chem. A 2012, 116, 3781-3793.

(19) Islam, M. A. Einstein-Smoluchowski Diffusion Equation: A Discussion. Phys. Scr. 2004, 70, 120.

(20) Frisch, M.; Trucks, G.; Schlegel, H.; Scuseria, G.; Robb, M.; Cheeseman, J.; Scalmani, G.; Barone, V.; Mennucci, B.; Petersson, G.; et al. Gaussian09, R. A., 1; Gaussian. Inc.: Wallingford, CT, 2009.

(21) Bauernschmitt, R.; Ahlrichs, R. Treatment of Electronic Excitations within the Adiabatic Approximation of Time Dependent Density Functional Theory. Chem. Phys. Lett. 1996, 256, 454-464.

(22) Stratmann, R. E.; Scuseria, G. E.; Frisch, M. J. An Efficient Implementation of Time-Dependent Density-Functional Theory for the Calculation of Excitation Energies of Large Molecules. J. Chem. Phys. 1998, 109, 8218-8224.

(23) Casida, M. E.; Jamorski, C.; Casida, K. C.; Salahub, D. R. Molecular Excitation Energies to High-Lying Bound States from TimeDependent Density-Functional Response Theory: Characterization and Correction of the Time-Dependent Local Density Approximation Ionization Threshold. J. Chem. Phys. 1998, 108, 4439-4449.

(24) Aidas, K.; Angeli, C.; Bak, K. L.; Bakken, V.; Bast, R.; Boman, L.; Christiansen, O.; Cimiraglia, R.; Coriani, S.; Dahle, P. The Dalton Quantum Chemistry Program System. Wiley Interdiscip. Rev.: Comput. Mol. Sci. 2014, 4, 269-284.
(25) Yanai, T.; Tew, D. P.; Handy, N. C. A New Hybrid ExchangeCorrelation Functional Using the Coulomb-Attenuating Method (CamB3lyp). Chem. Phys. Lett. 2004, 393, 51-57.

(26) Mennucci, B.; Tomasi, J. Continuum Solvation Models: A New Approach to the Problem of Solute's Charge Distribution and Cavity Boundaries. J. Chem. Phys. 1997, 106, 5151-5158.

(27) Miertuš, S.; Scrocco, E.; Tomasi, J. Electrostatic Interaction of a Solute with a Continuum. A Direct Utilizaion of Ab Initio Molecular Potentials for the Prevision of Solvent Effects. Chem. Phys. 1981, 55, 117-129.

(28) Barone, V.; Cossi, M.; Tomasi, J. A New Definition of Cavities for the Computation of Solvation Free Energies by the Polarizable Continuum Model. J. Chem. Phys. 1997, 107, 3210-3221.

(29) McClain, W. M. Excited State Symmetry Assignment through Polarized Two-Photon Absorption Studies of Fluids. J. Chem. Phys. 1971, 55, 2789-2796.

(30) Ohta, K.; Antonov, L.; Yamada, S.; Kamada, K. Theoretical Study of the Two-Photon Absorption Properties of Several Asymmetrically Substituted Stilbenoid Molecules. J. Chem. Phys. 2007, 127, 084504.

(31) Monson, P. R.; McClain, W. M. Polarization Dependence of the Two-Photon Absorption of Tumbling Molecules with Application to Liquid 1-Chloronaphthalene and Benzene. J. Chem. Phys. 1970, 53, 2937.

(32) Kamada, K.; Ohta, K.; Iwase, Y.; Kondo, K. Two-Photon Absorption Properties of Symmetric Substituted Diacetylene: Drastic Enhancement of the Cross Section near the One-Photon Absorption Peak. Chem. Phys. Lett. 2003, 372, 386-393.

(33) Neves, U. M.; De Boni, L.; Ye, Z.; Bu, X. R.; Mendonça, C. R. Two-Photon Absorption Spectra of Salen Dye Complexes with Azo Dyes. Chem. Phys. Lett. 2007, 441, 221-225.

(34) Alajarín, R; Burgos, C. Six-Membered Heterocycles: Quinoline and Isoquinoline. Modern Heterocyclic Chemistry; John Wiley \& Sons, Inc., 2011; pp 1527-1629.

(35) Kamada, K.; Namikawa, T.; Senatore, S.; Matthews, C.; Lenne, P.F.; Maury, O.; Andraud, C.; Ponce-Vargas, M.; Le Guennic, B.; Jacquemin, D. Boron Difluoride Curcuminoid Fluorophores with Enhanced Two-Photon Excited Fluorescence Emission and Versatile Living-Cell Imaging Properties. Chem.-Eur. J. 2016, 22, 5219-5232.

(36) Bonin, K. D.; McIlrath, T. J. Two-Photon Electric-Dipole Selection Rules. J. Opt. Soc. Am. B 1984, 1, 52-55.

(37) Kuzyk, M. G.; Dirk, C. W. Effects of Centrosymmetry on the Nonresonant Electronic Third-Order Nonlinear Optical Susceptibility. Phys. Rev. A 1990, 41, 5098.

(38) Albota, M.; Beljonne, D.; Brédas, J.-L.; Ehrlich, J. E.; Fu, J.-Y.; Heikal, A. A.; Hess, S. E.; Kogej, T.; Levin, M. D.; Marder, S. R. Design of Organic Molecules with Large Two-Photon Absorption Cross Sections. Science 1998, 281, 1653-1656.

(39) Pawlicki, M.; Collins, H. A.; Denning, R. G.; Anderson, H. L. Two-Photon Absorption and the Design of Two-Photon Dyes. Angew. Chem., Int. Ed. 2009, 48, 3244-3266.

(40) Lee, S.; Thomas, K. R. J.; Thayumanavan, S.; Bardeen, C. J. Dependence of the Two-Photon Absorption Cross Section on the Conjugation of the Phenylacetylene Linker in Dipolar Donor- BridgeAcceptor Chromophores. J. Phys. Chem. A 2005, 109, 9767-9774.

(41) Drobizhev, M.; Makarov, N. S.; Tillo, S. E.; Hughes, T. E.; Rebane, A. Two-Photon Absorption Properties of Fluorescent Proteins. Nat. Methods 2011, 8, 393-399. 


\title{
On the spectral line width broadening for simulation of the two-photon absorption cross-section of para-Nitroaniline in liquid environment
}

\author{
Tárcius N. Ramos ${ }^{\text {a }}$, Daniel L. Silva ${ }^{\mathrm{b}}$, Benedito J.C. Cabral ${ }^{\mathrm{c}}$, Sylvio Canuto ${ }^{\mathrm{a}, *}$ \\ a Universidade de Sao Paulo, Instituto de Fisica, Rua do Matão 1371, 05508-090 São Paulo, SP, Brazil \\ b Departamento de Ciências da Natureza, Matemática e Educação, Universidade Federal de São Carlos, Rod. Anhanguera - Km 174, $13600-970$ Araras, SP, Brazil

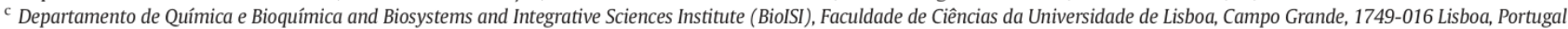

\section{A R T I C L E I N F O}

\section{Article history:}

Received 23 October 2019

Received in revised form 12 December 2019

Accepted 25 December 2019

Available online 02 January 2020

\section{Keywords:}

Two-photon absorption

Broadening effects

$\mathrm{S}-\mathrm{QM} / \mathrm{MM}$

Para-Nitroaniline

\begin{abstract}
A B S T R A C T
The calculated two-photon absorption (TPA) cross-section amplitude depends on the spectral line width $(\Gamma)$ of the transition and there is not a consensus on the most appropriate way to obtain the $\Gamma$ value. Usually it is arbitrarily defined or ambiguously estimated based on experimental data or using a transition energy variation determined from a quantum mechanics/molecular mechanics approach. In this study we compare different procedures to estimate $\Gamma$ values and different methods of spectral convolution to simulate TPA cross-section for para-Nitroaniline ( $\mathrm{pNA}$ ) in liquid environment. The effects of the liquid environment on the nonlinear absorption were approximated using Monte Carlo and Born-Oppenheimer Molecular Dynamics as well as the commonly used Polarizable Continuum Model. The different procedures adopted here provided large difference in the calculated TPA amplitudes, mainly because of the underestimate/overestimate of the homogeneous and/or inhomogeneous contributions to the spectral line width. Based on these difficulties, we suggest a new procedure in which the value assigned to $\Gamma$ is the one used to induce the total line width of the simulated TPA spectrum to reproduce the experimental one-photon absorption line width. Within this procedure the $\Gamma$ value, which becomes a fitting parameter $\left(\Gamma_{f i t}\right)$, represents part of the homogeneous contribution to the spectral line width. Finally, although time dependent density functional theory calculations reproduced only reasonably well the energy of the lowest-energy $\pi \rightarrow \pi^{*}$ transition of pNA, the fitting procedure here proposed provided the calculated TPA cross-section in good agreement with the available experimental data.
\end{abstract}

(c) 2018 Elsevier B.V. All rights reserved.

\section{Introduction}

Nonlinear effects in organic compounds have been largely studied in the last decades mainly because of improvement in the light sources and in the computational capabilities. Two-photon absorption (TPA) is a nonlinear process related to the imaginary part of the second order hyperpolarizability (or third order electric susceptibility) and depends on the square of the intensity of the incident light beam. In this process two photons (with equal or different energy values) are simultaneously absorbed by an atomic/molecular system that achieves a final excited state. With higher intensity and short pulse duration accessible in the current days, some undesired effects like thermal effects can be avoided and new technological applications are receiving attention. Three dimensional storage [1], fluorescence microscopy [2], microfabrication [3] and photodynamic therapy [4] are some examples of the wide range of applications employing TPA process. From the theoretical point of view, to calculate the TPA probabilities one possibility is to compute sums over ground-excited and excited-excited electronic

\footnotetext{
* Corresponding author.

E-mail address: canuto@if.usp.br (S. Canuto).
}

transitions which demand larger computational efforts. This approach is called Sum-Over-States and is often combined with semi empirical methods or in few-states approximations. Nowadays, employing second order Time Dependent perturbation approaches $[5,6]$ it is possible to reduce the computational costs and study TPA using more rigorous quantum chemical methods as Coupled Cluster or TD-DFT (Time Dependent Density Functional Theory). Beyond the computational demand, an additional and important parameter related to the spectral broadening of electronic transition is also a requisite to simulate the TPA cross-section. A Lorentzian function is often employed to represent the spectral broadening of an electronic transition and the related TPA cross-section depends on the full width at half maximum $(\Gamma)$ of the Lorentzian function. The difficulty to assign the $\Gamma$ value is not peculiar to the TPA cross-section and similar dependence of broadening functions is also presented in one-photon absorption (OPA) cross-section as well as in molar absorptivity.

For TPA cross-section calculations, usually an arbitrary value of $0.1 \mathrm{eV}$ is adopted for $\Gamma$. Some attempts have been made to describe the line width as an estimative from the transition energy variation [7] when a set of configurations is sampled from molecular simulation. A recent alternative to reproduce the asymmetric band shapes 
combines the homogeneous broadening from vibrational TPA spectra (which also need an empirical value for the spectral broadening function) with the inhomogeneous fluctuations of the transition energies obtained from rigid classical molecular simulations [8]. If an experimental value for $\Gamma$ is available it is possible to use this information to build the theoretical spectra for isolated dye and even in solvent using the Polarizable Continuum Model (PCM) $[9,10]$ to approximate the environment. Here, we are including the solvent molecules explicitly from sampled configurations obtained in molecular simulations. To obtain the maximum TPA cross-section we analysed five different spectral convolution procedures, they involve the experimental OPA $\Gamma$ value, the $\Gamma$ related to standard deviation of transition energies and a new suggestion of using a fit to assign the $\Gamma$ value. In this fitting procedure, the $\Gamma$ value for sampled configurations was chosen imposing that the full width at half maximum of the total spectrum $\Gamma_{t o t}$ reproduces a previously defined value. The molecules classified as non-centrosymmetric exhibit flexible electronic transition selection rules allowing the same excited state to be accessed by both linear and nonlinear absorption process. Considering this, a simple approximation can be done assuming the $\Gamma_{\text {tot }}$ value as the one obtained in experimental OPA analyses. This OPA based assumption allows simulating the TPA cross-section even if there is no data from TPA experiments. On the other hand, this initial and nonarbitrary guess should be a simple starting point to simulate the TPA cross-section when the target state is not accessed by linear absorption. The aim of the present work is to compare the results of TPA cross-section employing different procedures to set the $\Gamma$ value for an ensemble of configurations sampled from molecular simulations. The molecular simulations considered here include classical rigid Monte Carlo and quantum flexible Born Oppenheimer Molecular dynamics.

The para-Nitroaniline (pNA, Fig. 1) is a small molecule with donor and acceptor peripheral groups and large solvatochromic shift of $0.99 \mathrm{eV}$ from gas phase to aqueous solution [11]. Its spectroscopic properties were already largely studied using the quantum-chemical approaches, from the isolated case to supercritical environments conditions [12-21]. The solvatochromic shift has not been well reproduced in theoretical studies using the TD-DFT, but the CAMB3LYP functional showed a better compromise comparing with Coupled Cluster based results $[17,18,22]$. The TPA of the isolated pNA was already investigated using semi empirical, DFT, Coupled Cluster methods $[18,23,24]$ and including vibrational effects [25]. These previous theoretical calculations achieved values from $25.1 \mathrm{GM}$ to $107 \mathrm{GM}$ which are quite distant from the $8 \mathrm{GM}$ observed in dioxane [26]. Theoretical studies of TPA cross-sections for micro hydrated pNA [27] were also reported. We highlight the difficulties to compare theoretical values for the TPA cross-section because of the different values assumed for the $\Gamma$ parameter and for the constants in the TPA cross-section expression. The pNA shows low solubility and weak higher-order nonlinear signal, making it difficult to measure its TPA properties. Recently, its TPA cross-section in different liquids were rigorously measured by Wielgus and collaborators [26] opening new possibilities for theoretical investigations.

Hence, our investigation considered the pNA in three liquid environments: 1,4-dioxane (henceforth referred to as dioxane), dimethylsulfoxide (DMSO), and water. These solvents were included using PCM and discrete approximations (using the Monte Carlo sampling). Additional Born-Oppenheimer Molecular Dynamics (BOMD)

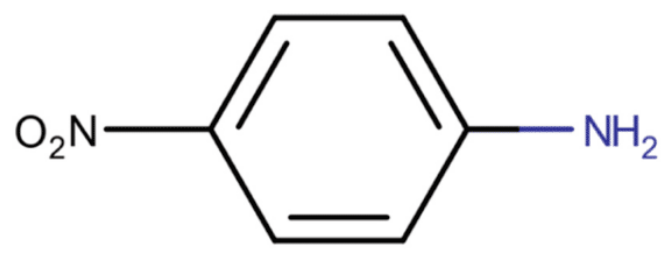

Fig. 1. Diagram of pNA molecule. were also carried out for pNA isolated and surrounded by explicit water molecules. Therefore, we analysed the ground state geometry, the transition energy and the TPA cross-section for the low energy absorption band. As we will see, the procedure of fitting the total spectra to reproduce the line width of the OPA showed the best agreement with experiment for the TPA cross-section and provided information about homogeneous broadening effects. Here we focus in obtaining the line width for better obtaining two-photon absorption crosssections and we have not made any attempt to reproduce the band shape.

\section{Theoretical approach}

All the geometries of the pNA were obtained by energy minimization calculations followed by second derivative analyses to confirm their minimum conditions at the level MP2/aug-cc-pVDZ. The solvation effects were included in the energy minimizations using the PCM, as implemented in Gaussian 09 [28] software, for three environments dioxane, DMSO and water. The dioxane and DMSO solvents have been chosen for the sake of availability of experimental data (TPA spectrum) in the literature [26]. Although there is no TPA experimental data in water, because of low water solubility, the water solvent was also assumed for comparison between classical and quantum molecular simulations. The economical Monte Carlo and the expensive BornOppenheimer Molecular Dynamics simulations were performed for such purpose.

Simulations with rigid molecules were performed in the DICE [29] program employing the Monte Carlo (MC) Metropolis [30] algorithm. After the simulations, a set of uncorrelated energy configurations were selected for Quantum Mechanics calculations, this approach developed in our group has been termed as the Sequential-Quantum Mechanics/ Molecular Mechanics (S-QM/MM) [31,32] and it has shown relative success describing a wide range of electronic properties including electronic polarization [33], absorption spectrum in liquids and complex environments such as supercritical water [19,34-36] and even protein ambient [37]. The Optimized Potential for Liquid Simulations-All Atom (OPLS-AA) $[38,39]$ were adopted as Lennard-Jones parameters for the molecular force fields of the solute. To account for the solute polarization the charges for the solute molecule were calculated in the presence of the solvent using the CHELPG [40] electronic density fit employing the MP2/aug-cc-pVDZ level set in combination with the PCM approximation. The rigid pNA geometries used in the MC simulations were obtained employing PCM approximation.

The force fields for the solvents were adopted using the following references: water (SPC/E) [41], DMSO [42,43] and dioxane [44]. Cubic boxes with periodic boundary conditions containing one pNA $+\mathrm{N}$ solvent molecules were previously thermalized and additional $2.5 \times 10^{8}$ MC steps were performed to produce configurations for S-QM/MM. The number $\mathrm{N}=1000$ of solvent molecules were defined for dioxane and water and $\mathrm{N}=500$ for DMSO. All the simulations were performed in the NPT ensemble with the temperature of $298.15 \mathrm{~K}$ and the pressure of $1 \mathrm{~atm}$. Hydrogen bonds were identified using both geometric and energetic criterion. This is necessary because it cannot be assured that all solvent molecules satisfying the geometric criterion are indeed forming a hydrogen bond with the solute $[45,46]$. In this work hydrogen bonds were identified from the radial distribution functions and the pairwise interacting diagram. The criterion obtained were that the distance between the acceptor-donor atoms are $<3.5 \AA$, the angle formed by donor-acceptor-hydrogen atoms is $<40^{\circ}$ and the solute-solvent energy interaction is lower than $-3.0 \mathrm{kcal} / \mathrm{mol}$ for water and $-1.5 \mathrm{kcal} / \mathrm{mol}$ for DMSO. The procedures to define these criteria are fully described in references $[45,46]$. These geometric and energetic criteria are necessary to ensure that the nearby solvent molecules are really involved in solute-solvent hydrogen bonds $[47,48]$. A set of 100 statistically uncorrelated configurations $[32,49]$ was selected from each simulation and submitted to QM calculations. When only the electrostatic effects of 
the solvents are aimed in the calculations, an efficient alternative for the QM calculations is the Average Solvent Electrostatic Configuration (ASEC) [50]. In the ASEC, the solvent molecules are approximated by the atomic point charges of the force field. In this case instead of taking the average of all configurations an average configuration is used and only one QM calculation is made giving the same result [50].

The trajectories from the ab initio BOMD simulations of the pNA isolated and solvated in water were obtained previously [20]. These have been analysed here to obtain information about the TPA spectroscopy line broadening and to compare the results with the classical MC simulation. Two simulations were carried out in the NVT ensemble: the isolated pNA with the Martyna and Tuckerman Poisson solver for nonperiodic boundary conditions [51] and the pNA surrounded by 96 water molecules, in a cubic box of size $\mathrm{L}=14.59 \AA \AA$ in periodic boundary conditions. The target temperature was $298 \mathrm{~K}$. Fifty picoseconds of trajectories were calculated and the last 25 ps were used in QM calculations. The BLYP-D3 [52-54] functional was employed combined with the DZVP-MOLOPT- SR [55] basis set in the CP2K [56] program. The values of -0.786 a.u. and 0.393 a.u. for the respective charge of the water's oxygen and hydrogens were used when the point charge approximation for the solvent was adopted. For more details of the simulations see reference [20].

To compare experimental and theoretical values for the TPA crosssection is important to notice that there are some definitions and constants for the TPA expression as well as different experimental sets [22]. The following expressions for TPA cross-section are comparable to one-beam Z-Scan technique [57]. The mathematical representation of the TPA cross-section $\left(\sigma_{g f}\right)$, given in atomic units [22,58,59], for each electronic transition between ground $(g)$ and final $(f)$ excited state is

$\sigma_{g f}\left(\omega_{g f}, \delta_{g f} ; \Gamma_{f}\right)=\frac{4 \pi^{3}}{c^{2}} \omega^{2} g\left(2 \omega, \omega_{g f} ; \Gamma_{f}\right) \delta_{g f}$

where $\omega_{g f}$ is the energy of the electronic transition, $\omega$ is the energy of the incident photon $\left(\omega=\omega_{g f} / 2\right)$ and $c$ is the light speed. The spectral broadening function $g\left(2 \omega, \omega_{g f} ; \Gamma_{f}\right)$ is assumed as a Lorentzian shape (expressed in Eq. (2)) with a full-width at half-maximum damping constant $\Gamma_{f}$. The TPA transition probability $\delta_{g f}$ in the case of a linearly polarized beam source is defined in Eq. (3a) and (3b).

$g\left(2 \omega, \omega_{g f} ; \Gamma_{f}\right)=\frac{1}{2 \pi} \frac{\Gamma_{f}}{\left(2 \omega-\omega_{g f}\right)^{2}+0.25 \Gamma_{f}^{2}}$

$\delta_{g f}=\frac{1}{15} \sum_{\alpha \beta}\left\{S_{\alpha \alpha}^{g f}\left(S_{\beta \beta}^{g f}\right)^{*}+2 S_{\alpha \beta}^{g f}\left(S_{\alpha \beta}^{g f}\right)^{*}\right\}$

$S_{\alpha \beta}^{g f}=\sum_{k}\left\{\frac{\left\langle g\left|\mu_{\alpha}\right| k\right\rangle\left\langle k\left|\mu_{\beta}\right| f\right\rangle}{\omega_{g k}-\omega}+\frac{\left\langle g\left|\mu_{\beta}\right| k\right\rangle\left\langle k\left|\mu_{\alpha}\right| f\right\rangle}{\omega_{g k}-\omega}\right\}$

Here, the Greek symbols represent the Cartesian coordinates and the star $\left({ }^{*}\right)$ the complex conjugate. The $k$ index represents intermediate electronic states. The conversion factor from atomic units (a.u.) to Göppert-Mayer (GM) is 1.896788 and $1 \mathrm{GM}$ represents $10^{-50} \mathrm{~cm}^{4} \mathrm{~s}$ / photon.

We are analysing five different ways to build TPA spectra from a set of statistically uncorrelated configurations sampled from molecular simulations of pNA in different solvents, they are: P1 - setting $\Gamma_{f}$ value as the experimental line width of the OPA band $\left(\Gamma_{\exp }\right)$ for each lowestenergy TPA transition assumed and using the average values of $\overline{\omega^{2} \delta_{g f}}$ and $\overline{\omega_{g f}}$ terms computed by taking into account all uncorrelated configurations; P2 - setting $\Gamma$ values for each transition based on the variation of the transition energy and is given by $\Gamma_{f}=\Gamma_{s t d}=\sqrt{8 \ln 2} * \sigma_{S T D}\left(\omega_{g f}\right)$, where $\sigma_{S T D}$ is the standard deviation, and using the average values determined from the set of statistically uncorrelated configurations for $\overline{\omega^{2} \delta_{g f}}$ and $\overline{\omega_{g f}}$; P3 - setting $\Gamma_{f}$ value as the experimental line width of the OPA band $\left(\Gamma_{\exp }\right)$ for each lowest-energy TPA transition calculated and computing the averaged spectrum; P4 - setting $\Gamma_{f}=\Gamma_{\text {std }}$ (as defined for P2) and computing the averaged spectrum; P5 - setting one value $\left(\Gamma_{f i t}\right)$ for $\Gamma_{f}$ in such way that the line width of the simulated TPA spectrum is induced to reproduce the line width of the experimental OPA band/spectrum $\left(\Gamma_{\text {exp }}\right)$ and computing the average spectrum. In Fig. 2 is presented an illustration in order to elucidate the differences between the different procedures employed in this work to simulate the TPA spectrum. We also defined the $\Gamma_{\text {tot }}$ as the value of the line width obtained from the final spectra, in this way the P5 always will provide the $\Gamma_{\text {tot }}=\Gamma_{\text {exp. }}$. The experimental line width is obtained from a gaussian fit and a factor of $1 / 1.418$ was applied to convert to Lorentzian equivalent value.

The broadening effects in the absorption process has two contributions, the homogeneous and the inhomogeneous. The homogeneous contributions are ascribed to the solute flexibility while the inhomogeneous term is related to the environment effects on the solute electronic structure. These contributions are not unrelated and the environment effects obtained from the simulation includes the absorption broadening mostly from the inhomogeneous contribution. Different assumptions of the $\Gamma_{f}$ values can result in lack or excess of the homogeneous and/or the inhomogeneous contributions. When the solute is considered rigid during the molecular simulations (as in our MC simulations) the homogeneous contribution is inhibited. Therefore, in P2 and P4 $\left(\Gamma_{f}=\Gamma_{s t d}\right)$ the homogeneous part is neglected. On the other hand, in P3 and P4 the inhomogeneous part is included twice - once from the average spectra and another by $\Gamma_{f}=\Gamma_{\exp }(\mathrm{P} 3)$ or by $\Gamma_{f}=\Gamma_{\text {std }}(\mathrm{P} 4)$. The fit performed for P5 is a way to avoid such lack or excess of the broadening contributions and should result in the best compromise of the broadening effects. For flexible solute simulations the P1 and P2 should include at once both contributions (homogeneous and inhomogeneous) since $\Gamma_{f}=\Gamma_{\text {exp }}$ for P1 and because of both broadening effects are present in the $\Gamma_{f}=\Gamma_{\text {std }}$ for P2 procedure. The P3 and P4 include both broadening effects twice, once as P1 and P2 and another one from the average spectrum. A synthesis of these considerations is presented in Table 1.

A set of configurations with explicit solvent molecules were submitted for TPA calculations, making it difficult to employ expensive wavefunction methods and, therefore, the DFT model was chosen in the following calculations. The TPA spectroscopic properties were computed using the long range coulomb attenuated CAM-B3LYP functional [60] which is appropriate for charge transfer systems and has been reported as a good alternative to wavefunction methods. The TPA calculations were carried out using the Time Dependent Quadratic Response formalism as implemented in Dalton software [5,6] with CAM-B3LYP functional using the aug-cc-pVDZ basis set for pNA in all calculations. When solvent molecules were included explicitly, we used the aug-ccpVDZ basis set for water molecules and $6-31+G^{*}$ for dioxane and DMSO molecules. For large number of explicit water molecules we reduce the basis set to $6-31+\mathrm{G}^{*}$.

\section{Results and discussions}

\subsection{Ground state properties}

We obtained the ground state geometries for the pNA isolated as well as in solvents (dioxane, DMSO and water) approximated by PCM. The minimum energy conditions were confirmed without negative frequencies founded in Hessian analyses for all environments. All geometries obtained are essentially planar with the hydrogens bonded to the nitrogen forming a pyramidal structure (out of the ring plane). Due to the similarities among the geometries, only the minimum energy geometry obtained in water environment and its dipole moment vector are 


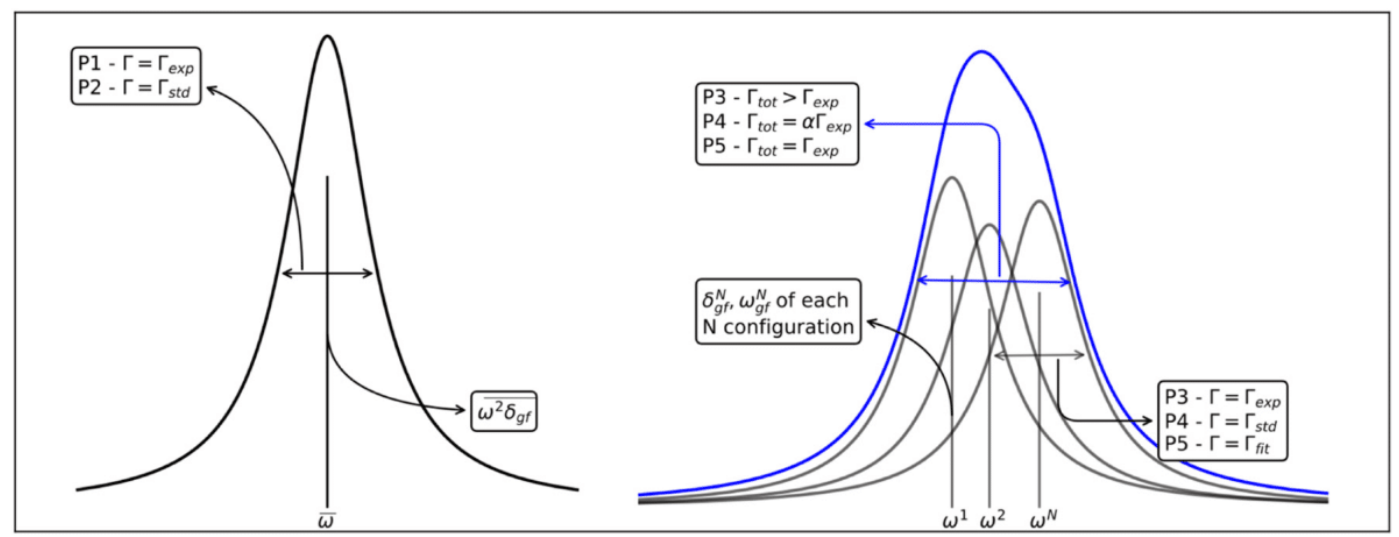

Fig. 2. The effect of the different procedures in the total line width.

presented in Fig. 3. As described in the previous section, for the MC simulations rigid geometries are used whereas in the BOMD they are flexible. The average geometry obtained from the BOMD water simulation in water (WATER-BOMD) is in good agreement with the geometry obtained for the solute in water environment using the PCM approximation.

The ground state dipole moments of the isolated pNA were computed employing MP2 (6.2 D) and CAM-B3LYP functional (6.8 D), these results are in good agreement with previous calculations (5.2 D to 7.0 D) $[11,20,25]$. Solvent effects were included employing PCM and ASEC approximations and the results are presented in Table 2. The ground state dipole moment increases with the solvent polarity (as expected for push-pull systems) and they are larger using ASEC than using PCM. Besides, the average dipole moment from WATER-BOMD was calculated using point charge approximation for the water molecules and its result is equivalent to that obtained using the ASEC approximation of the MC simulation. The experimental ground state dipole moment is estimated between $5.2 \mathrm{D}$ and $6.5 \mathrm{D}[16,61]$ for pNA in dioxane.

From the radial distribution functions, we observed that the first solvation shell contains 16 solvent molecules of dioxane and DMSO, while for water this number increases to 33 and 39 molecules for water-MC and WATER-BOMD simulations, respectively. The average (and the maximum) numbers of molecules making hydrogen bonds are 1.9 (3), 3.3 (6) and 3.8 (6) respectively for the simulation of pNA in DMSO, in water-MC and in water-BOMD. In Fig. 4 present the hydrogen bonds formed between the solute and the solvent. In fact, this is a superposition of all the hydrogen bonds formed such it is possible to see the configuration space occupied by the hydrogen bonded water (top) and
DMSO (bottom). The solvent dioxane did not show hydrogen bond structures as expected for a nonpolar and nonprotic solvent.

\subsection{Two-photon absorption spectra}

The lowest-energy $\pi \rightarrow \pi^{*}$ transition is the second absorption transition, but it is the most intense transition for both OPA and TPA process. Analysis of the orbitals shows that this transition is dominated by the promotion from the HOMO to the LUMO orbitals. These orbitals are similar for isolated and solvated calculations and in Fig. 5 are presented such orbitals obtained for isolated pNA. As the same transition is promoted in both linear and nonlinear processes, we may assume the same value for OPA and TPA spectral broadening to calculate the TPA cross-section and simulate the TPA spectra. For isolated pNA we calculated a value of $4.31 \mathrm{eV}$ for the transition energy ( $4.25 \mathrm{eV}$ is the experimental result [11]) and $6.0 \mathrm{GM}$ for the TPA cross-section (using $\Gamma=$ $0.45 \mathrm{eV}$ extracted from reference [11]). Using the PCM approximation we observed a solvatochromic shift of $0.74 \mathrm{eV}$ (from isolated to water) that is smaller than the experimental value of $0.99 \mathrm{eV}$. The solvatochromic shift observed using the ASEC approximation is only $0.43 \mathrm{eV}$, indicating that the electrostatic interaction accounts for half of the solvatochromic effects.

The amplitudes of TPA cross-section obtained employing the PCM approximation were 14.2 GM (dioxane), 38.5 GM (DMSO) and $26.0 \mathrm{GM}$ (water) that are more than twice of the experimental value ( 8 GM and 12 GM respectively for dioxane and DMSO). Using the ASEC approximation the TPA amplitudes calculated were below the experimental measures, the values were $7.5 \mathrm{GM}$ (dioxane), 8.6 GM

Table 1

Overview of the different procedures adopted including the definition of the $\Gamma_{f}$ values, the TPA cross-section $(\sigma)$ equations and the respective broadening effects contribution expected for each pair of definitions, $\Gamma_{f}$ and $\sigma$.

\begin{tabular}{|c|c|c|c|c|}
\hline Procedure & $\Gamma_{f}$ & $\begin{array}{l}\text { TPA } \\
\text { cross-section - } \sigma\end{array}$ & $\begin{array}{l}\text { Inhomogeneous } \\
\text { contribution }\end{array}$ & $\begin{array}{l}\text { Homogeneous } \\
\text { contribution }\end{array}$ \\
\hline P1 & $\begin{array}{l}\Gamma_{\text {exp }} \text { (from } \\
\text { experimental } \\
\text { OPA spectrum) }\end{array}$ & $\frac{4 \pi^{3}}{c^{2}} \sum_{f} \overline{\omega^{2} \delta_{g f}} g\left(2 \omega, \overline{\omega_{g f}} ; \Gamma_{f}\right)$ & Included & $\begin{array}{l}\text { Included - MC } \\
\text { Included - BOMD }\end{array}$ \\
\hline P2 & $\begin{array}{l}\Gamma_{\text {std }} \text { (from } \\
\mathrm{QM} \text { calculations) }\end{array}$ & & Included & $\begin{array}{l}\text { Not included - MC } \\
\text { Included - BOMD }\end{array}$ \\
\hline P3 & $\begin{array}{l}\Gamma_{\exp }(\text { from } \\
\text { experimental } \\
\text { OPA spectrum) }\end{array}$ & $\frac{4 \pi^{3}}{c^{2}} \frac{1}{N_{\text {conf }}} \sum_{f, N}\left(\omega^{N}\right)^{2} \delta_{g f}^{N} g\left(2 \omega, \omega_{g f}^{N} ; \Gamma_{f}\right)$ & Included twice & $\begin{array}{l}\text { Included - MC } \\
\text { Included twice - } \\
\text { BOMD }\end{array}$ \\
\hline P4 & $\begin{array}{l}\Gamma_{\text {std }} \text { (from } \\
\mathrm{QM} \text { calculations) }\end{array}$ & & Included twice & $\begin{array}{l}\text { Not included - MC } \\
\text { Included twice - } \\
\text { BOMD }\end{array}$ \\
\hline P5 & $\begin{array}{l}\Gamma_{f i t} \text { (from the fitting } \\
\text { of the experimental } \\
\text { OPA spectrum) }\end{array}$ & & Included & Included (via $\left.\Gamma_{f i t}\right)$ \\
\hline
\end{tabular}




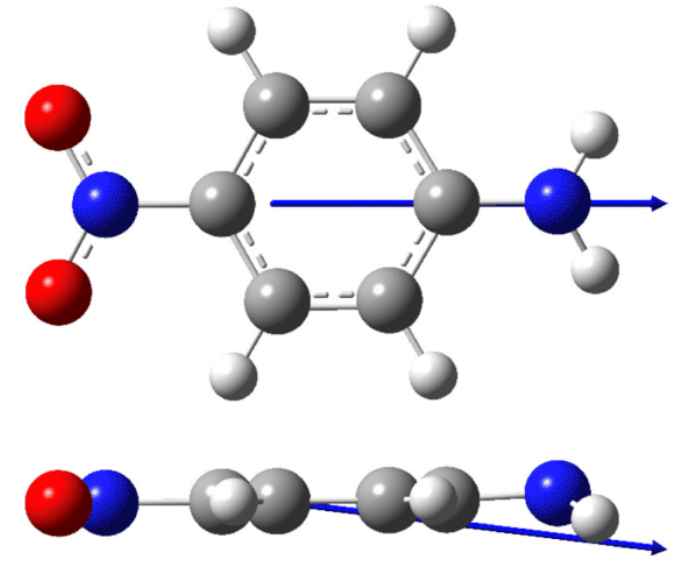

Fig. 3. Minimum energy geometry obtained in water and its dipole moment vector.

(DMSO) and 5.4 GM (water). Therefore, only the solute-solvent electrostatic interaction did not reproduce well the solvent effects on the TPA cross-section, what suggests that a description of the solute-solvent interaction beyond the electrostatic is necessary.

To improve the environment description for the $\mathrm{QM}$ calculations we now include explicitly some of the nearest solvent molecules as follow: the 2 nearest dioxane molecules (named as 2-DIOXANE); the DMSO molecules that make hydrogen bond (HB-DMSO); the 5 nearest DMSO molecules (5-DMSO); the water molecules that perform hydrogen bond (HB-WATER); the 15 nearest water molecules (15-WATER). We also included additional 250 nearest solvent molecules as point charges in the 100 sampled uncorrelated energy configurations. For the BOMD simulation we included the water molecules that make hydrogen bond (HB-WATER-BOMD) plus the remaining water molecules treated as point charges in a set of 200 snapshots equally separated in time by $125 \mathrm{fs}$.

The TPA spectra were next simulated using the five procedures described before in the theoretical session and we obtained the TPA cross-section maximum and the respective transition energy values $\left(\omega_{\max }\right)$ from the simulated spectra and the results are reported in Table 3. The transition energy corresponding to the maximum absorption obtained from simulated spectra employing P1-P5 analyses showed variations lower than $0.03 \mathrm{eV}$ for simulations with a rigid geometry and lower than $0.1 \mathrm{eV}$ for the flexible case. As described in the theoretical section the TPA cross-section depends on the line width value $(\Gamma)$ and such different definitions (see Table 1) can include or not the contributions of the (in)homogeneous broadening effects. The amplitudes of the TPA cross-section calculated varied more than twice due to the different $\Gamma$ values, evidencing the necessity to apply an adequate approach based on the physical meaning of $\Gamma$ and the limitation of the molecular simulation to obtain reasonable TPA amplitudes for a solvated molecule. This is particularly important when the geometries are considered rigid during the molecular simulations. Because the use of a set of sampled configurations reproduces only the inhomogeneous contribution for the spectral broadening it is physically justifiable to use a fitting parameter $\left(\Gamma_{f i t}\right)$ as a complement to reproduce the total spectral

Table 2

Ground state permanent dipole moments (in Debye).

\begin{tabular}{lllll}
\hline & DIOXANE & DMSO & WATER & WATER-BOMD \\
\hline PCM - MP2 & 7.13 & 8.54 & 8.58 & - \\
PCM - CAM-B3LYP & 7.82 & 9.35 & 9.39 & - \\
ASEC - CAM-B3LYP & 8.14 & 9.60 & 9.73 & $11.0 \pm 1.7^{\text {a }}$ \\
\hline
\end{tabular}

a Result obtained approximating the solvent as point charges for flexible WATER-BOMD simulation.
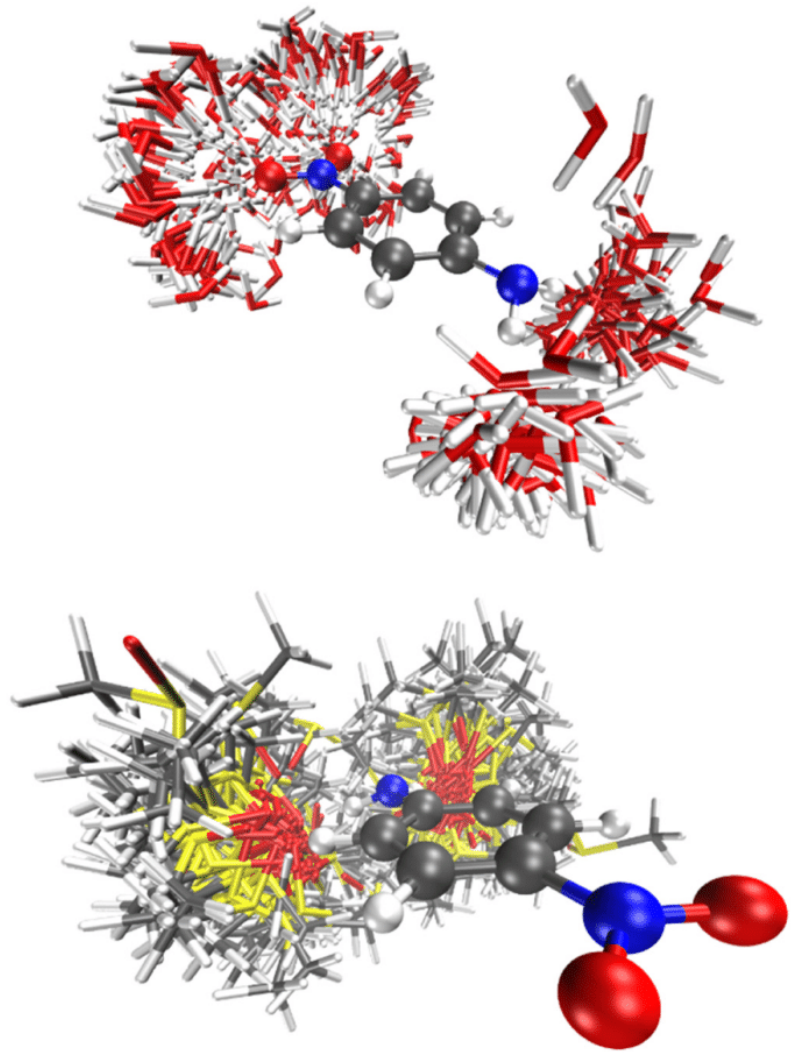

Fig. 4. Illustration of the hydrogen bonds formed by the pNA and solvent molecules. The figure superimposes configurations to show the space occupied by the hydrogen bonds.

broadening observed in the experimental spectra. Such fitting parameter would then represent somehow the homogeneous contribution. This is the main idea of the P5 scheme, which, in this study, showed the best description of the TPA cross-section when compared with the accessible experimental data. The lowest excited state of isolated pNA is of $n-\pi^{*}$ type and it can invert the order with the intense lowenergy transition (in reason of environments effect) providing undesired effects on the $\Gamma_{\text {std }}$ calculation. To avoid this effect, we need to distinguish these two transitions for each snapshot in order to properly compute the average values of the $\overline{\omega^{2} \delta_{g f}}$ and $\overline{\omega_{g f}}$ parameters and $\Gamma_{\text {std }}$ when applicable. Although visual analyses of the molecular orbitals
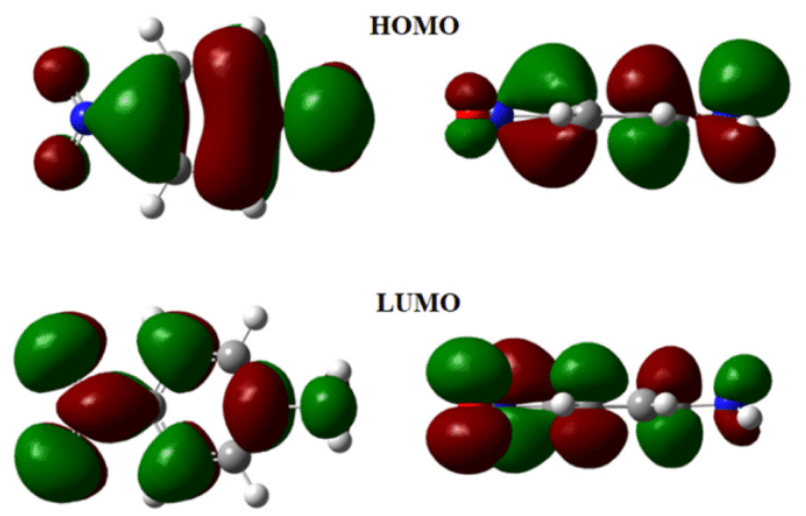

LUMO

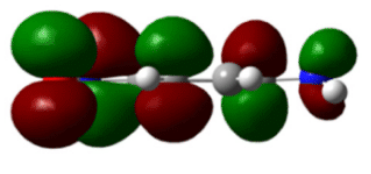

Fig. 5. HOMO-LUMO orbitals involved in the first intense transition for one- and twophoton processes in isolated pNA. 
Table 3

TPA cross-section for pNA in solution. The transition energies $\left(\omega_{\max }\right)$ and the total line widths $\left(\Gamma_{\text {tot }}\right)$ are in $\mathrm{eV}$ and the TPA cross-sections $(\sigma)$ are in GM. The experimental values $(\mathrm{EXP})$ were obtained from references $[26,64]$. The $\Gamma_{f i t}$ values obtained for $\mathrm{P} 5$ are presented in parenthesis.

\begin{tabular}{|c|c|c|c|c|c|c|c|c|c|c|c|c|c|c|c|c|c|c|}
\hline & \multicolumn{3}{|c|}{ 2-DIOXANE } & \multicolumn{3}{|c|}{ HB-DMSO } & \multicolumn{3}{|c|}{ 5-DMSO } & \multicolumn{3}{|c|}{ HB-WATER } & \multicolumn{3}{|c|}{ 15-WATER } & \multicolumn{3}{|c|}{ HB-WATER-BOMD } \\
\hline & $\omega_{\max }$ & $\Gamma_{t o t}$ & $\sigma$ & $\omega_{\max }$ & $\Gamma_{t o t}$ & $\sigma$ & $\omega_{\max }$ & $\Gamma_{\text {tot }}$ & $\sigma$ & $\omega_{\max }$ & $\Gamma_{t o t}$ & $\sigma$ & $\omega_{\max }$ & $\Gamma_{t o t}$ & $\sigma$ & $\omega_{\max }$ & $\Gamma_{\text {tot }}$ & $\sigma$ \\
\hline $\mathrm{PCM}^{\mathrm{a}}$ & 4.01 & 0.39 & 14.2 & 3.59 & 0.34 & 38.5 & - & - & - & 3.57 & 0.52 & 26.0 & - & - & - & - & - & - \\
\hline $\operatorname{ASEC}^{\mathrm{a}, \mathrm{b}}$ & 4.11 & 0.39 & 7.5 & 3.91 & 0.34 & 8.6 & - & - & - & 3.88 & 0.52 & 5.4 & - & - & - & 3.69 & 0.52 & 5.3 \\
\hline P1 & 4.04 & 0.43 & 8.7 & 3.77 & 0.38 & 10.7 & 3.74 & 0.37 & 10.3 & 3.77 & 0.56 & 6.2 & 3.74 & 0.57 & 6.2 & 3.72 & 0.70 & 3.8 \\
\hline P2 & 4.05 & 0.18 & 20.9 & 3.77 & 0.18 & 20.6 & 3.74 & 0.20 & 17.8 & 3.76 & 0.25 & 13.3 & 3.73 & 0.28 & 12.3 & 3.73 & 0.61 & 4.4 \\
\hline P3 & 4.04 & 0.48 & 8.1 & 3.79 & 0.44 & 9.5 & 3.75 & 0.46 & 8.9 & 3.79 & 0.65 & 5.7 & 3.75 & 0.67 & 5.6 & 3.67 & 0.88 & 3.2 \\
\hline P4 & 4.03 & 0.27 & 15.5 & 3.78 & 0.35 & 14.7 & 3.75 & 0.37 & 12.6 & 3.79 & 0.45 & 9.4 & 3.75 & 0.50 & 8.51 & 3.63 & 0.78 & 3.8 \\
\hline P5 & 4.04 & $0.39(0.30)$ & 10.1 & 3.79 & $0.34(0.21)$ & 13.4 & 3.75 & $0.34(0.20)$ & 12.8 & 3.79 & $0.52(0.37)$ & 7.3 & 3.75 & $0.52(0.33)$ & 7.7 & 3.65 & $0.52(0.06)$ & 6.7 \\
\hline EXP & 3.51 & 0.39 & 8 & 3.18 & 0.34 & 12 & 3.18 & 0.34 & 12 & 3.26 & 0.52 & - & 3.26 & 0.52 & - & 3.26 & 0.52 & - \\
\hline
\end{tabular}

a The PCM and ASEC results are presented only once for each solvent.

${ }^{b}$ Represents the result describing the solvent as point charges for flexible WATER-BOMD simulation using the P5.

involved can often help to characterize an electronic transition, sometimes it is not possible to safely classify it into $n \rightarrow \pi^{*}$ or $\pi \rightarrow \pi^{*}$ type. Such issue is the reason for P1 total line width to be larger than the experimental (see Fig. 6). A comparison for the $\Gamma_{\text {tot }}$ value shows that $\mathrm{P} 2<\mathrm{P} 4<\mathrm{P} 1<\mathrm{P} 3$, this information can be easily visualised in Fig. 6 and it is corroborated by the amount of (in)homogeneous contributions presented in Table 1.

The amplitude of TPA cross-section increased from dioxane to DMSO in $18 \%$ and $10 \%$ for $\mathrm{P} 1$ and $\mathrm{P} 3$ respectively, while the $\mathrm{P} 2$ and $\mathrm{P} 4$ showed an inverse tendency with a decrease of $15 \%$. The P5 provides increase of $27 \%$ in the TPA cross-section from dioxane to DMSO, which is our best value compared with $50 \%$ increase observed in experiments. All simulated spectra are shown in Fig. 7. Increasing the number of explicit solvent molecules, the agreement of the theoretical results for transition energies and TPA cross-section with the experimental values is slightly improved, although the small differences observed did not justify the additional computational effort. However, another interesting feature observed for P5 is the good agreement in TPA cross-section between economical MC and expensive BOMD simulations in water, what it was not observed for P1-P4. All these results are numerically presented in Table 3. Additional TPA cross-section analyses were performed employing the P5 for HB-DMSO and HB-WATER configurations using the hybrid B3LYP functional. The values of the TPA cross-section

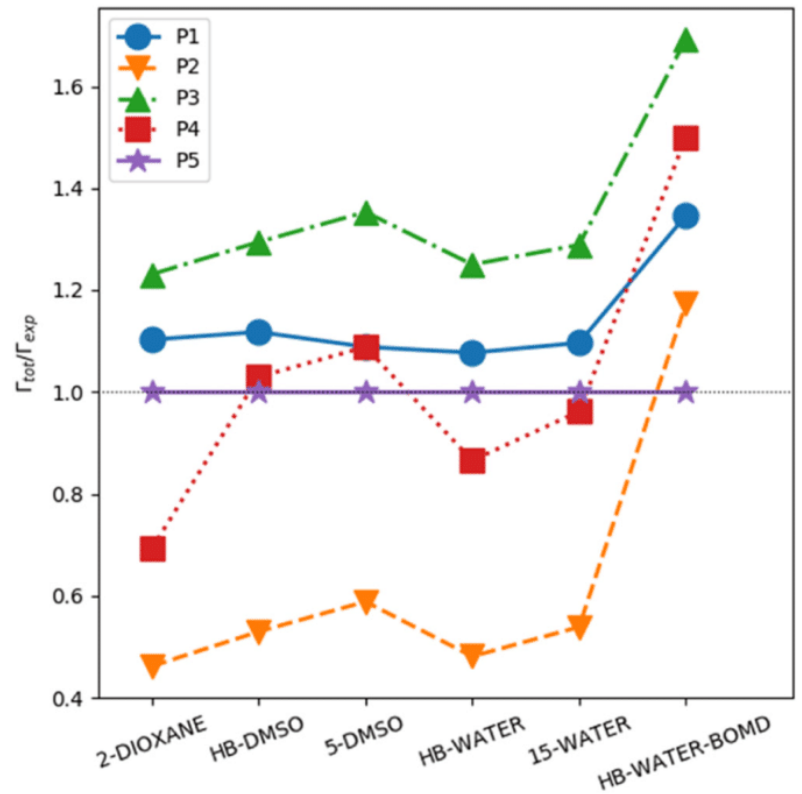

Fig. 6. Comparison of the total line width obtained from simulated TPA spectra and the OPA experiment. maximum obtained were $\sim 30 \%$ lower than the ones obtained using the CAM-B3LYP. However, the comparison of the $\Gamma_{\text {fit }}$ values among these two functionals are in good agreement showing small differences of 0.02 and $0.03 \mathrm{eV}$ for HB-DMSO and HB-WATER respectively. Such similarities of the $\Gamma_{f i t}$ values indicate stability in the estimation of the broadening effects. The use of the CAM-B3LYP method in calculating TPA is well documented $[22,62]$. A natural aspect to be considered is the possible influence of dispersion effects. The use of appended terms in regular DFT calculations has shown some relative success but it has not been aimed or tested for the case of TPA. An additional aspect of practical relevance is that dispersion corrected DFT functional are not available for time dependent quadratic response calculations. In addition, it is known that when using wave functions that are antisymmetric with respect to the entire system, solute and the solvent, the delocalization of the orbitals may include partial dispersion [63].

As already mentioned, we assumed that $\Gamma_{f i t}$ describes (at least semi quantitatively) the homogeneous contribution to the spectral broadening of the average spectra. Thus, it is to be expected that its amplitude is more significant in the case that the TPA spectrum is simulated based on the statistically uncorrelated configurations coming from the MC simulation. This behaviour can be observed comparing the $\Gamma_{\text {fit }}$ values of $0.37 \mathrm{eV}$ (HB-WATER) obtained with MC - without homogeneous contribution - with the value of $0.06 \mathrm{eV}$ (HB-WATER-BOMD) - which includes homogeneous effects. Two complementary analyses were performed in order to improve the description of the (in)homogeneous broadening. The transition energies and the TPA properties from HB-WATER-BOMD configurations were calculated considering only the geometrical changes of the pNA (without the inclusion of any water molecules), called here as WATER-ISO. Another BOMD simulation was performed for the isolated pNA and 200 configurations from the last 24 ps were equally separated in each $125 \mathrm{fs}$, it was termed as ISOLATED. Analysing the ISOLATED and WATER-ISO results we can better understand the environment perturbation effects on the homogeneous broadening. The water perturbations in homogeneous broadening were analysed employing the P2 because $\Gamma_{f}$ is defined only by simulations analyses without previous information (as OPA line width for example). The transition energy and TPA cross-section decrease in $0.15 \mathrm{eV}$ and in $3.4 \mathrm{GM}$ from ISOLATED to WATER-ISO. When the explicit water molecules were included (HB-WATER-BOMD) there was an additional $0.28 \mathrm{eV}$ in the red shift in the transition energy providing a total red shift of $0.43 \mathrm{eV}$. The water presence reduces the TPA cross-section calculated to less than half achieving the value of $4.4 \mathrm{GM}$. The results were summarized in Table 4 . The values of total line width calculated for WATER-ISO and ISOLATED cases are close to the value found for $\Gamma_{f i t}$ obtained in MC simulations, corroborating that the application of $\mathrm{P} 5$ not only makes it possible to obtain a simulated TPA spectrum more consistent with the experimental one but can also provide information about the homogeneous contribution to the spectral broadening observed. 


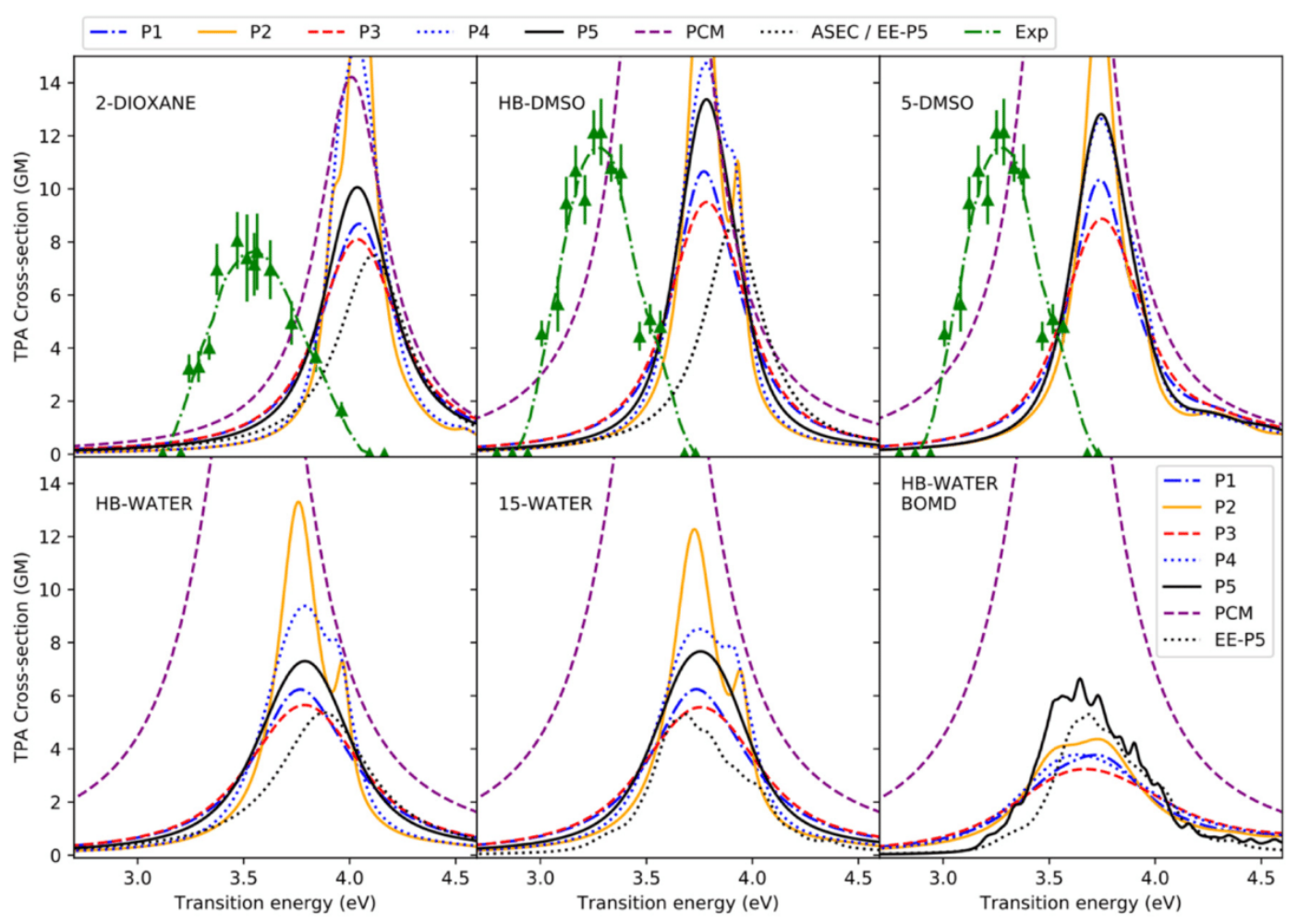

Fig. 7. Simulated TPA spectra obtained employing the five procedures adopted and the PCM and ASEC solvent approximations. The transition energy is equal to 2 times the photon energy. The experimental points were extracted from reference [26].

\section{Conclusions}

The two-photon absorption (TPA) cross-section of a molecule is an important property for applications based on the TPA process. The TPA cross-section depends on the line width $(\Gamma)$ of the electronic transition and, therefore, the value of the width is important and should not be arbitrarily ascribed. In the present work, different procedures using different approaches for defining the $\Gamma$ value combined with different methods of spectral convolution have been used to simulate TPA cross-section of para-Nitroaniline (pNA) in a liquid environment. The large differences obtained for the calculated TPA amplitudes, among the different procedures adopted, are explained by either the underestimation or overestimation of the homogeneous and/or inhomogeneous broadening contributions for the spectral line width of the lowestenergy transition of pNA.

The new procedure discussed here to define the $\Gamma$ value, imposes the total line width of the simulated spectrum to reproduce the experimental one-photon absorption (OPA) line width and, therefore, it avoids overestimating/underestimating of the broadening contributions on the convoluted TPA spectrum. Such assumption was done as a simple approximation because the intense transition can be accessed by both linear and nonlinear absorption processes. However, this assumption should not be a good approximation when such condition is not

Table 4

Transition energy $\left(\omega_{\max }\right)$ in $\mathrm{eV}$, total line width $\left(\Gamma_{\text {tot }}\right)$ in $\mathrm{eV}$ and TPA absorption cross-section $(\sigma)$ in GM from BOMD simulations. We emphasize the absence of experimental data for pNA in water solvent.

\begin{tabular}{llll}
\hline & $\omega_{\max }$ & $\Gamma_{\text {tot }}$ & $\sigma$ \\
\hline ISOLATED & 4.16 & 0.28 & 10.3 \\
WATER-ISO & 4.01 & 0.33 & 6.9 \\
HB-WATER-BOMD & 3.73 & 0.61 & 4.4 \\
\hline
\end{tabular}

achieved. The fitting procedure, that consists in fitting the experimental OPA data using sampled configurations from molecular simulations and takes the $\Gamma$ value as a fitting parameter, provided the more accurate theoretical TPA cross-section amplitude of pNA in solution among all the procedures here analysed.

For the cases in which no experimental OPA data is available, our results point that a procedure combining rigid and flexible simulations could be applied as a good alternative to simulate the TPA spectrum. In this procedure the theoretical $\Gamma$ value is defined as the standard deviation of the TD-DFT transition energy as computed using sampled configurations from a flexible quantum molecular dynamic simulation performed for the isolated solute molecule. Such $\Gamma$ value is then used to convolute the TPA spectrum using the sampled configurations of a rigid simulation.

In addition, our results also show that a procedure defining the $\Gamma$ value as the standard deviation of the transition energy in TD-DFT calculations performed for the solvated solute molecule using sampled configurations from rigid or flexible (classical or quantum) molecular simulations provides reasonable TPA cross-section amplitudes. Therefore, although using this procedure the $\Gamma$ value cannot be further discussed physically, it can also be an economical (single molecular simulation) alternative procedure in the absence of experimental OPA.

Finally, our results indicate that the use of the fitting procedure to estimate the $\Gamma$ value is the best choice when $\mathrm{QM} / \mathrm{MM}$ methodology is adopted to include the environment effects in the study of the TPA process of a molecule in a liquid environment. Although this is not an extensive study, here we presented some alternatives to calculate the TPA cross-section and estimating the $\Gamma$ value. We believe that these results will be useful in further calculations concerning the simulation of the TPA cross-section of newly designed compounds in solution. Before concluding, it should be noted that the case studied here, of pNA in different solvents such as dioxane, DMSO and water are not specific and 
the procedures suggested here can, of course, be generalized for other molecules and other environments.

\section{CRediT authorship contribution statement}

Tárcius N. Ramos: Writing - original draft, Writing - review \& editing, Formal analysis, Investigation. Daniel L. Silva: Writing - original draft, Writing - review \& editing, Formal analysis. Benedito J.C. Cabral: Writing - original draft, Writing - review \& editing, Formal analysis, Investigation. Sylvio Canuto: Writing - original draft, Writing - review \& editing, Formal analysis.

\section{Declaration of competing interest}

The authors declare that they have no known competing financial interests or personal relationships that could have appeared to influence the work reported in this paper.

\section{Acknowledgement}

This work was supported by grant 2015/14189-3 from São Paulo Research Foundation (FAPESP). The computational facilities were supported by INCT-FCX, BioMol and FAPESP 2015/20032-0. BJCC was supported by Fundação para a Ciência e a Tecnologia (FCT, Portugal) through BioISI funding project UID/Multi/04046/2013.

\section{References}

[1] D.A. Parthenopoulos, P.M. Rentzepis, Three-dimensional optical storage memory, Science 245 (1989) 843-845, https://doi.org/10.1126/science.245.4920.843.

[2] W. Denk, J.H. Strickler, W.W. Webb, Two-photon laser scanning fluorescence microscopy, Science 248 (1990) 73-76, https://doi.org/10.1126/science.2321027.

[3] S. Kawata, H.B. Sun, T. Tanaka, K. Takada, Finer features for functional microdevices, Nature 412 (2001) 697-698, https://doi.org/10.1038/35089130.

[4] J.D. Bhawalkar, G.S. He, P.N. Prasad, Nonlinear multiphoton processes in organic an polymeric materials, Rep. Prog. Phys. 59 (1996) 1041-1070, https://doi.org/10 1088/0034-4885/59/9/001

[5] K. Aidas, C. Angeli, K.L. Bak, V. Bakken, R. Bast, L. Boman, O. Christiansen, R. Cimiraglia, S. Coriani, P. Dahle, E.K. Dalskov, U. Ekström, T. Enevoldsen, J.J. Eriksen, P. Ettenhuber, B. Fernández, L. Ferrighi, H. Fliegl, L Frediani, K. Hald, A. Halkier, C. Hättig, H. Heiberg, T. Helgaker, A.C. Hennum, H. Hettema, E. Hjertenaes, S. Høst, IHättig, H. Heiberg, T. Helgaker, A.C. Hennum, H. Hettema, E. Hjertenaes, S. Høst, I.-
M. Høyvik, M.F. Iozzi, B. Jansík, H.J.A. Jensen, D. Jonsson, P. Jørgensen, J. Kauczor, S Kirpekar, T. Kjaergaard, W. Klopper, S. Knecht, R. Kobayashi, H. Koch, J. Kongsted, A. Krapp, K. Kristensen, A. Ligabue, O.B. Lutnaes, J.I. Melo, K.V. Mikkelsen, R.H Myhre, C. Neiss, C.B. Nielsen, P. Norman, J. Olsen, J.M.H. Olsen, A. Osted, M. Packer, F. Pawlowski, T.B. Pedersen, P.F. Provasi, S. Reine, Z. Rinkevicius, T.A. Ruden, K. Ruud, V.V. Rybkin, P. Sałek, C.C.M. Samson, A.S de Merás, T. Saue, S.P.A. Sauer, B. Schimmelpfennig. K. Sneskov, A.H. Steindal, K.O. Sylvester-Hvid, P.R. Taylor, A.M. Teale, E.I. Tellgren, D.P. Tew, A.J. Thorvaldsen, L. Thøgersen, O. Taylor, A.M. Teale, E.I. Tellgren, D.P. Tew, A.J. Thorvaldsen, L. Thøgersen, O.
Vahtras, M.A. Watson, D.J.D. Wilson, M. Ziolkowski, H. Ágren, The Dalton quantum chemistry program system, Wiley Interdiscip. Rev. Comput. Mol. Sci. 4 (2014) 269-284, https://doi.org/10.1002/wcms.1172.

[6] Dalton, a Molecular Electronic Structure Program, Release Dalton2016.1, see http:// daltonprogram.org 2016.

[7] D.L. Silva, N.A. Murugan, J. Kongsted, Z. Rinkevicius, S. Canuto, H. Ågren, The role of molecular conformation and polarizable embedding for one- and two-photon absorption of disperse orange 3 in solution, J. Phys. Chem. B 116 (2012) 8169-8181, https://doi.org/10.1021/jp3032034.

[8] J. Bednarska, R. Zaleśny, G. Tian, N.A. Murugan, H. Ågren, W. Bartkowiak, Nonempirical simulations of inhomogeneous broadening of electronic transitions in solution: predicting band shapes in one- and two-photon absorption spectra of chalcones, Molecules 22 (2017) 1643, https://doi.org/10.3390/molecules22101643.

[9] S. Miertuš, E. Scrocco, J. Tomasi, Electrostatic interaction of a solute with a continuum. A direct utilizaion of $\mathrm{AB}$ initio molecular potentials for the prevision of solvent effects, Chem. Phys. 55 (1981) 117-129, https://doi.org/10.1016/0301-0104(81) 85090-2.

[10] R. Cammi, B. Mennucci, Linear response theory for the polarizable continuum model, J. Chem. Phys. 110 (1999) 9877-9886, https://doi.org/10.1063/1.478861.

[11] S. Millefiori, G. Favini, A. Millefiori, D. Grasso, Electronic spectra and structure of nitroanilines, Spectrochim. Acta A: Mol. Spectrosc. 33 (1977) 21-27, https://doi. org/10.1016/0584-8539(77)80143-8.

[12] T. Yasukawa, T. Kimura, M. Uda, An AM1 study of the effects of intermolecular interactions on hyperpolarizability of p-nitroanilines, Chem. Phys. Lett. 169 (1990) 259-262, https://doi.org/10.1016/0009-2614(90)85197-K.

[13] Y. Luo, H. Ågren, O. Vahtras, P. Jørgensen, The hyperpolarizability dispersion of paranitroaniline, Chem. Phys. Lett. 207 (1993) 190-194, https://doi.org/10.1016/00092614(93)87013-S
[14] W. Bartkowiak, J. Lipiński, Solvent effect on the nonlinear optical properties of paranitroaniline studied by Langevin dipoles-Monte Carlo (LD/MC) approach, Comput Chem. 22 (1998) 31-37, https://doi.org/10.1016/S0097-8485(97)00030-2.

[15] L Jensen, P.T. van Duijnen, The first hyperpolarizability of p-nitroaniline in 1,4-dioxane: a quantum mechanical/molecular mechanics study, J. Chem. Phys. 123 (2005), 074307. https://doi.org/10.1063/1.1999633.

[16] L.C.T. Shoute, R. Helburn, A.M. Kelley, Solvent effects on the resonance Raman and hyper-Raman spectra and first hyperpolarizability of N,N-dipropyl-p-nitroaniline, J. Phys. Chem. A 111 (2007) 1251-1258, https://doi.org/10.1021/jp066599s.

[17] J.J. Eriksen, S.P.A. Sauer, K.V. Mikkelsen, O. Christiansen, H.J.A. Jensen, J. Kongsted, Failures of TDDFT in describing the lowest intramolecular charge-transfer excitation in para-nitroaniline, Mol. Phys. 111 (2013) 1235-1248, https://doi.org/10.1080/ 00268976.2013 .793841$.

[18] D.H. Friese, C. Hättig, K. Ruud, Calculation of two-photon absorption strengths with the approximate coupled cluster singles and doubles model CC2 using the resolution-of-identity approximation, Phys. Chem. Chem. Phys. 14 (2012) 1175-1184, https://doi.org/10.1039/c1cp23045j.

[19] M.H. Cardenuto, K. Coutinho, B.J.C. Cabral, S. Canuto, Electronic properties in supercritical fluids, Adv. Quantum Chem. 71 (2015) 323-339, https://doi.org/10.1016/bs. aiq.2015.03.006.

[20] B.J.C. Cabral, K. Coutinho, S. Canuto, A first-principles approach to the dynamics and electronic properties of p-nitroaniline in water, J. Phys. Chem. A 120 (2016) 3878-3887, https://doi.org/10.1021/acs.jpca.6b01797.

[21] H. Reis, A. Grzybowski, M.G. Papadopoulos, Computer simulation of the linear and nonlinear optical susceptibilities of p-nitroaniline in cyclohexane, 1,4-dioxane, and tetrahydrofuran in quadrupolar approximation. I. Molecular polarizabilities and hyperpolarizabilities, J. Phys. Chem. A 109 (2005) 10106-10120, https://doi.org/ 10.1021/jp052875b.

[22] M.T.P. Beerepoot, D.H. Friese, N.H. List, J. Kongsted, K. Ruud, Benchmarking twophoton absorption cross sections: performance of CC2 and CAM-B3LYP, Phys. Chem. Chem. Phys. 17 (2015) 19306-19314, https://doi.org/10.1039/C5CP03241E.

[23] D. Beljonne, W. Wenseleers, E. Zojer, Z. Shuai, H. Vogel, S.J.K. Pond, J.W. Perry, S.R Marder, J.L. Brédas, Role of dimensionality on the two-photon absorption response of conjugated molecules: the case of octupolar compounds, Adv. Funct. Mater. 12 (2002) 631-641, https://doi.org/10.1002/1616-3028(20020916)12:9<631::AIDADFM631>3.0.CO;2-W

[24] E. Rudberg, P. Sałek, T. Helgaker, H. Ågren, Calculations of two-photon chargetransfer excitations using Coulomb-attenuated density-functional theory, J. Chem. Phys. 123 (2005), 184108. https://doi.org/10.1063/1.2104367.

[25] R. Zaleśny, G. Tian, C. Hättig, W. Bartkowiak, H. Ågren, Toward assessment of density functionals for vibronic coupling in two-photon absorption: a case study of 4 nitroaniline, J. Comput. Chem. 36 (2015) 1124-1131, https://doi.org/10.1002/jcc. 23903.

[26] M. Wielgus, J. Michalska, M. Samoc, W. Bartkowiak, Two-photon solvatochromism III: experimental study of the solvent effects on two-photon absorption spectrum of p-nitroaniline, Dyes Pigments 113 (2015) 426-434, https://doi.org/10.1016/j. dyepig.2014.09.009.

[27] K.D. Nanda, A.I. Krylov, The effect of polarizable environment on two-photon absorption cross sections characterized by the equation-of-motion coupledcluster singles and doubles method combined with the effective fragment potential approach, J. Chem. Phys. 149 (2018), 164109. https://doi.org/10.1063/ 1.5048627 .

[28] M.J. Frisch, G.W. Trucks, H.B. Schlegel, G.E. Scuseria, M.A. Robb, J.R. Cheeseman, G. Scalmani, V. Barone, B. Mennucci, G.A. Petersson, H. Nakatsuji, M. Caricato, X. Li, H.P. Hratchian, A.F. Izmaylov, J. Bloino, G. Zheng, J.L. Sonnenberg, M. Hada, M. Ehara, K. Toyota, R. Fukuda, J. Hasegawa, M. Ishida, T. Nakajima, Y. Honda, O. Kitao, H. Nakai, T. Vreven, J.A. Montgomery Jr., J.E. Peralta, F. Ogliaro, M. Bearpark, J.J. Heyd, E. Brothers, K.N. Kudin, V.N. Staroverov, R. Kobayashi, J. Normand, K. Raghavachari, A. Rendell, J.C. Burant, S.S. Iyengar, J. Tomasi, M. Cossi, N. Rega, J.M Millam, M. Klene, J.E. Knox, J.B. Cross, V. Bakken, C. Adamo, J. Jaramillo, R

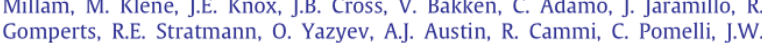
Ochterski, R.L. Martin, K. Morokuma, V.G. Zakrzewski, G.A. Voth, P. Salvador, J. Dannenberg, S. Dapprich, A.D. Daniels, O. Farkas, J.B. Foresman, J.V. Ortiz, J. Cioslowski, D.J. Fox, Gaussian09 Revision C.01, 2010.

[29] K. Coutinho, S. Canuto, DICE(v2.9): A Monte Carlo Program for Molecular Liquid Simulation, University of Sao Paulo, Brazil, 2013.

[30] N. Metropolis, A.W. Rosenbluth, M.N. Rosenbluth, A.H. Teller, E. Teller, Equation of state calculations by fast computing machines, J. Chem. Phys. 21 (1953) 1087-1092, https://doi.org/10.1063/1.1699114.

[31] W.R. Rocha, V.M. Martins, K. Coutinho, S. Canuto, Solvent effects on the electronic absorption spectrum of formamide studied by a sequential Monte Carlo/quantum mechanical approach, Theor. Chem. Accounts 108 (2002) 31-37, https://doi.org/ 10.1007/s00214-002-0353-y.

[32] K. Coutinho, S. Canuto, M.C. Zerner, Monte Carlo-quantum mechanics study of the solvatochromic shifts of the lowest transition of benzene, J. Chem. Phys. 112 (2000) 9874-9880, https://doi.org/10.1063/1.481624.

[33] R. Rivelino, B.J.C. Cabral, K. Coutinho, S. Canuto, Electronic polarization in liquid acetonitrile: a sequential Monte Carlo/quantum mechanics investigation, Chem. Phys. Lett. 407 (2005) 13-17, https://doi.org/10.1016/j.cplett.2005.03.049.

[34] K. Coutinho, R.C. Guedes, B.J. Costa Cabral, S. Canuto, Electronic polarization of liquid water: converged Monte Carlo-quantum mechanics results for the multipole moments, Chem. Phys. Lett. 369 (2003) 345-353, https://doi.org/10.1016/S00092614(02)02026-2

[35] V. Ludwig, K. Coutinho, S. Canuto, A Monte Carlo-quantum mechanics study of the lowest n-pi* and pi-pi* states of uracil in water, Phys. Chem. Chem. Phys. 9 (2007) 4907-4912, https://doi.org/10.1039/b704335j. 
[36] D.L. Silva, N.A. Murugan, J. Kongsted, H. Ågren, S. Canuto, Self-aggregation and optical absorption of stilbazolium merocyanine in chloroform, J. Phys. Chem. B 118 (2014) 1715-1725, https://doi.org/10.1021/jp411178h.

[37] Y. Orozco-Gonzalez, M. Manathunga, M. del C. Marín, D. Agathangelou, K.-H. Jung, F. Melaccio, N. Ferré, S. Haacke, K. Coutinho, S. Canuto, M. Olivucci, An average solvent electrostatic configuration protocol for $\mathrm{QM} / \mathrm{MM}$ free energy optimization: implementation and application to rhodopsin systems, J. Chem. Theory Comput. 13 mentation and application to rhodopsin systems, J. Chem.
(2017) 6391-6404, https://doi.org/10.1021/acs.jctc.7b00860.

[38] J. Pranata, S.G. Wierschke, W.L. Jorgensen, OPLS potential functions for nucleotide bases. Relative association constants of hydrogen-bonded base pairs in chloroform, J. Am. Chem. Soc. 113 (1991) 2810-2819, https://doi.org/10.1021/ja00008a002.

[39] W.L. Jorgensen, D.S. Maxwell, J. Tirado-Rives, Development and testing of the OPLS all-atom force field on conformational energetics and properties of organic liqu

[40] C.M. Breneman, K.B. Wiberg, Determining atom-centered monopoles from molecular electrostatic potentials. The need for high sampling density in formamide conformational analysis, J. Comput. Chem. 11 (1990) 361-373, https://doi.org/10.1002/jcc. 540110311.

[41] H.J.C. Berendsen, J.R. Grigera, T.P. Straatsma, The missing term in effective pair potentials, J. Phys. Chem. 91 (1987) 6269-6271, https://doi.org/10.1021/j100308a038

[42] Y. Zheng, R.L. Ornstein, A molecular dynamics and quantum mechanics analysis of the effect of DMSO on enzyme structure and dynamics: subtilisin, J. Am. Chem. Soc. 118 (1996) 4175-4180, https://doi.org/10.1021/ja9539195.

[43] A. Vishnyakov, A.P. Lyubartsev, A. Laaksonen, Molecular dynamics simulations of dimethyl sulfoxide and dimethyl sulfoxide-water mixture, J. Phys. Chem. A 105 (2001) 1702-1710, https://doi.org/10.1021/jp0007336.

[44] M. Luhmer, J. Reisse, Molecular dynamics simulation study of the NMR relaxation of xenon-131 dissolved in 1,3-dioxane and 1,4-dioxane, J. Magn. Reson. Ser. A 115 (1995) 197-205, https://doi.org/10.1006/jmra.1995.1167.

[45] K. Coutinho, S. Canuto, The sequential Monte Carlo-quantum mechanics methodology. Application to the solvent effects in the stokes shift of acetone in water, J. Mol. ogy. Application to the solvent effects in the stokes shift of acetone in water, J. Mol.
Struct. THEOCHEM 632 (1-3) (2003) 235-246, https://doi.org/10.1016/S0166-1280 Struct. THEOCHE

[46] V. Manzoni, M.L. Lyra, K. Coutinho, S. Canuto, Comparison of polarizable continuum model and quantum mechanics/molecular mechanics solute electronic polarization: study of the optical and magnetic properties of diazines in water, J. Chem. Phys. 135 (14) (2011), 144103. https://doi.org/10.1063/1.3644894.

[47] S. Canuto, K. Coutinho, From hydrogen bond to bulk: solvation analysis of the $\mathrm{n}-\pi^{*}$ transition of formaldehyde in water, Int. J. Quantum Chem. 77 (2000) 192-198, https://doi.org/10.1002/(SICI) 1097-461X(2000)77:1<192::AIDQUA18>3.0.CO;2-2.

[48] K. Coutinho, B.J.C. Cabral, S. Canuto, Can larger dipoles solvate less? Solute-solvent hydrogen bond and the differential solvation of phenol and phenoxy, Chem. Phys. Lett. 399 (2004) 534-538, https://doi.org/10.1016/j.cplett.2004.10.063.

[49] K. Coutinho, S. Canuto, Solvent effects from a sequential Monte Carlo - quantum mechanical approach, Adv. Quantum Chem 1997, pp. 89-105, https://doi.org/10.1016/ S0065-3276(08)60209-9.
[50] K. Coutinho, H.C. Georg, T.L. Fonseca, V. Ludwig, S. Canuto, An efficient statistically converged average configuration for solvent effects, Chem. Phys. Lett. 437 (2007) 148-152, https://doi.org/10.1016/j.cplett.2007.02.012.

[51] G.J. Martyna, M.E. Tuckerman, A reciprocal space based method for treating long range interactions in ab initio and force-field-based calculations in clusters, J. Chem. Phys. 110 (1999) 2810-2821, https://doi.org/10.1063/1.477923.

[52] A.D. Becke, Density-functional thermochemistry. III. The role of exact exchange, J. Chem. Phys. 98 (1993) 5648-5652, https://doi.org/10.1063/1.464913.

[53] C. Lee, W. Yang, R.G. Parr, Development of the Colle-Salvetti correlation-energy formula into a functional of the electron density, Phys. Rev. B 37 (1988) 785-789, https://doi.org/10.1103/PhysRevB.37.785.

[54] T. Risthaus, S. Grimme, Benchmarking of London dispersion-accounting density functional theory methods on very large molecular complexes, J. Chem. Theory Comput. 9 (2013) 1580-1591, https://doi.org/10.1021/ct301081n.

[55] J. VandeVondele, J. Hutter, Gaussian basis sets for accurate calculations on molecular systems in gas and condensed phases, J. Chem. Phys. 127 (2007), 114105. https:// doi.org/10.1063/1.2770708.

[56] J. VandeVondele, M. Krack, F. Mohamed, M. Parrinello, T. Chassaing, J. Hutter, Quickstep: fast and accurate density functional calculations using a mixed Gaussian and plane waves approach, Comput. Phys. Commun. 167 (2005) 103-128, https://doi. org/10.1016/j.cpc.2004.12.014.

[57] M. Sheik-Bahae, A.A. Said, T.-H. Wei, D.J. Hagan, E.W. Van Stryland, Sensitive measurement of optical nonlinearities using a single beam, IEEE J. Quantum Electron. 26 (1990) 760-769, https://doi.org/10.1109/3.53394.

[58] P.R. Monson, W.M. McClain, Polarization dependence of the two-photon absorption of tumbling molecules with application to liquid 1-chloronaphthalene and benzene. J. Chem. Phys. 53 (1970) 29-37, https://doi.org/10.1063/1.1673778.

[59] W.M. McClain, Excited state symmetry assignment through polarized two-photon absorption studies of fluids, J. Chem. Phys. 55 (1971) 2789-2796, https://doi.org/ 10.1063/1.1676494.

[60] T. Yanai, D.P. Tew, N.C. Handy, A new hybrid exchange-correlation functional using the Coulomb-attenuating method (CAM-B3LYP), Chem. Phys. Lett. 393 (2004) 51-57, https://doi.org/10.1016/j.cplett.2004.06.011.

[61] H.K. Sinha, P.C.P. Thomson, K. Yates, Excited state dipole moments and polarizabilities of some aromatic alkenes and alkynes, Can. J. Chem. 68 (1990) 1507-1513, https://doi.org/10.1139/v90-232.

[62] M.T.P. Beerepoot, M.M. Alam, J. Bednarska, W. Bartkowiak, K. Ruud, R. Zaleśny, Benchmarking the performance of exchange-correlation functionals for predicting two-photon absorption strengths, J. Chem. Theory Comput. 14 (7) (2018) 3677-3685, https://doi.org/10.1021/acs.jctc.8b00245.

[63] S. Canuto, K. Coutinho, M.C. Zerner, Including dispersion in configuration interaction-singles calculations for the spectroscopy of chromophores in solution, J. Chem. Phys. 112 (17) (2000) 7293-7299, https://doi.org/10.1063/1.481332.

[64] S.A. Kovalenko, R. Schanz, V.M. Farztdinov, H. Hennig, N.P. Ernsting, Femtosecond relaxation of photoexcited para-nitroaniline: solvation, charge transfer, internal conversion and cooling, Chem. Phys. Lett. 323 (2000) 312-322, https://doi.org/10. 1016/S0009-2614(00)00432-2 